NATIONAL LABORATORY

\title{
LABORATORY DIRECTED RESEARCH AND DEVELOPMENT PROGRAM
}

FY 2007 ANNUAL REPORT

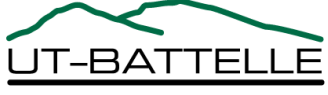




\section{DOCUMENT AVAILABILITY}

Reports produced after January 1, 1996, are generally available free via the U.S. Department of Energy (DOE) Information Bridge.

Web site http://www.osti.gov/bridge

Reports produced before January 1, 1996, may be purchased by members of the public from the following source.

National Technical Information Service

5285 Port Royal Road

Springfield, VA 22161

Telephone 703-605-6000 (1-800-553-6847)

TDD 703-487-4639

Fax 703-605-6900

E-mail info@ntis.fedworld.gov

Web site http://www.ntis.gov/support/ordernowabout.htm

Reports are available to DOE employees, DOE contractors, Energy Technology Data Exchange (ETDE) representatives, and International Nuclear Information System (INIS) representatives from the following source.

Office of Scientific and Technical Information

P.O. Box 62

Oak Ridge, TN 37831

Telephone 865-576-8401

Fax 865-576-5728

E-mail reports@osti.gov

Web site http://www.osti.gov/contact.html

This report was prepared as an account of work sponsored by an agency of the United States Government. Neither the United States government nor any agency thereof, nor any of their employees, makes any warranty, express or implied, or assumes any legal liability or responsibility for the accuracy, completeness, or usefulness of any information, apparatus, product, or process disclosed, or represents that its use would not infringe privately owned rights. Reference herein to any specific commercial product, process, or service by trade name, trademark, manufacturer, or otherwise, does not necessarily constitute or imply its endorsement, recommendation, or favoring by the United States Government or any agency thereof. The views and opinions of authors expressed herein do not necessarily state or reflect those of the United States Government or any agency thereof. 
Oak Ridge National Laboratory

\section{LABORATORY DIRECTED RESEARCH AND DEVELOPMENT PROGRAM FY 2007 ANNUAL REPORT}

March 2008

Prepared by OAK RIDGE NATIONAL LABORATORY

P.O. Box 2008

Oak Ridge, Tennessee 37831-6285 managed by UT-BATTELLE LLC for the U.S. DEPARTMENT OF ENERGY under Contract DE-AC05-00OR22725 



\section{CONTENTS}

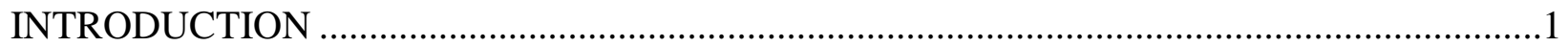

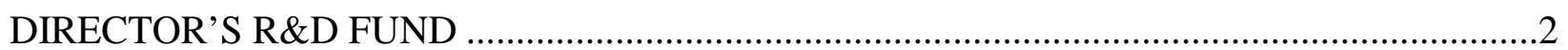

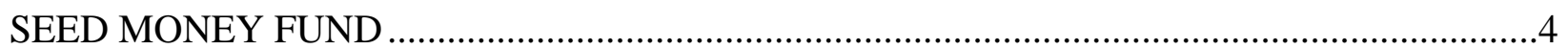

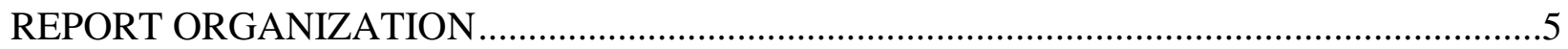

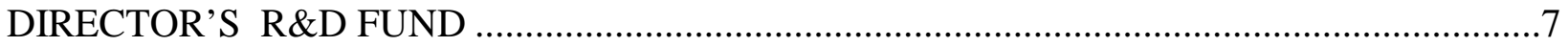

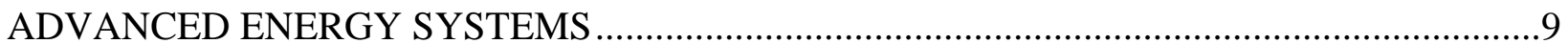

00233: Novel Carbon Materials for Advanced Energy Storage ...........................................9

00005: Multicomponent Fuel Spray Simulation Tools for Alternative Fuels .......................10

00234: Nanocomposite Dielectrics: New Smart Materials for

Electric Power Applications and the Advanced Grid ............................................ 12

00243: Experimental Optimization of Advanced Stellarator Confinement.........................13

00034: Advanced Nuclear Fuel Examination and Testing ................................................ 15

00036: A Hybrid Hydrogen Storage-Generation System Based on Bifunctional

Nanostructured Photocatalysts........................................................................ 16

00037: Modular Utility-Scale Power Converters and Controllers for the

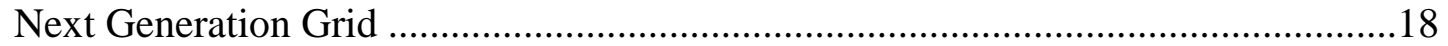

00038: Alternative Feedstocks for the Petrochemical Industry from

No-Sulfur-Added Biomass Lignins ............................................................... 19

00039: Developing a Science Base for Fuel Reprocessing Separations

in the Global Nuclear Energy Partnership ........................................................20

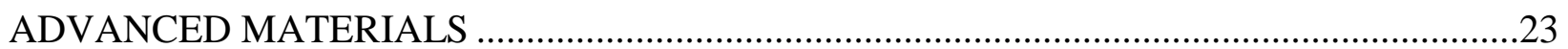

00220: High-Resolution Imaging of Biological Samples in a Wet Environment .................23

00224: Probing the Boundary between Imaging Microscopy and Spectroscopy:

Toward the Exploration of Single Particles by Nuclear Magnetic

Resonance Spectroscopy .....................................................................................24

00002: Synthesis and Neutron-Scattering Characterization of Ordered

Self-Assembled Polymer Nanostructures and Biomembranes ................................25

00229: Taming Electronic Spins in Conjugated Polymers for Photovoltaic

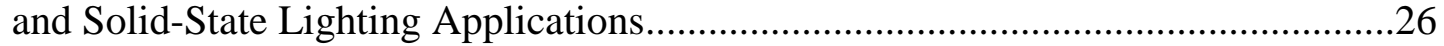

00239: Nanocrystalline/Amorphous Silicon Thin-Film Composite for Stable, High-Efficiency Photovoltaic Applications...........................................................27

00017: Nanoparticle Phase Change Materials: The Nanoscale Science Basis

for Gigajoule Energy Storage

00018: Apertureless Near-Field Desorption/Ionization Mass Spectrometry for Nanoscale Chemical Imaging at Atmospheric Pressure ......................................30

00027: Fundamental Mechanisms of Self-Assembly of Ordered Nanostructures in Heterogeneous Ceramic Materials 
00029: Imaging Energy Materials In Operandi with Atomic Resolution

Scanning-Transmission Electron Microscopy

00030: Antiferroelectric Thin-Film Capacitors for Ultrafast High-Power

Energy Storage.

00031: Energy Flow and Conversion on the Molecular Level: A View of Molecular

Photoelectromechanical Machines .35

00032: Nanostructured Thermoelectrics for Power Generation: Smaller Is Cooler...

00041: Smart Materials Toward a New Paradigm of Super-Efficient Separations Using only Energy Input: Conformational Switching Based on Magnetic Nanoparticles

00042: Design of Point Defect Trapping Centers in Nanostructured Nickel for Advanced Nuclear Applications

00045: Molecular Fragment Databases for De Novo Structure-Based Design

00050: Structure of Fluids Confined in Nanoporous Materials Using

Neutron Scattering.....

NATIONAL SECURITY

00223: Enhanced Cognizance of Evolving Threat Situations via

Knowledge Discovery from Disparate Data.

00047: Detection and Identification of Bacteria and Viruses Including Stealth and

Genetically Modified Organisms

00226: Design and Synthesis of Novel Infrared-Active Nanophosphors...

00237: Ensuring Dynamic Power Grid Stability: Integrated Electric and Information Grid Modeling

00046: Combustion of Nanostructured Metal Fuels: Towards Designing Optimized

Combustion Chambers.

00024: NanoEPower - Nanocatalytic Direct-Fuel Thermoelectric Generator.........................51

00028: A Novel Process of Thick Nanocomposite Surfaces for Defense Applications..........52

00035: Cognitive Radio for Transformational Logistics ........................................................53

NEUTRON SCIENCES.

00201: In Situ, Time-Resolved Neutron Diffraction Study of Materials Behavior under Severe Thermomechanical Deformation

00222: Time-Resolved Analyses of Microstructure in Advanced Materials under High Magnetic Fields Using Neutrons

00001: Infrastructure Development for Neutron Scattering for Biomembranes and Biomimetic Membranes.

00004: Small-Angle Neutron Scattering Investigations and Computational Modeling of Creep Cavitation in Nanoparticle-Strengthened Materials .62

00245: Use of Small Angle Neutron Scattering (SANS) to Study Complex Systems ...........63

00013: High-Temperature, High-Pressure Studies of Dynamics of Fluids in Nanopores using the SNS Backscattering Spectrometer.... .65

00015: A Robust Polymer Scaffold System for BioInspired Membranes

00019: Probing Molecular Interaction between Microbial-Cell Protein and Mineral Surfaces with Neutrons 
00022: High-Throughput Neutron Crystallography for Macromolecular Structure, Function

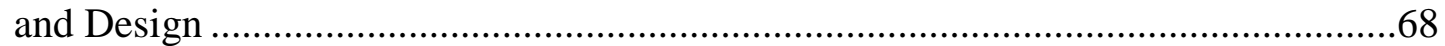

00023: Magnetic Structure Under Simultaneous High-Temperature/Ultra-HighPressure Conditions .........................................................................................69

00025: A Helicon Ion Source for the Spallation Neutron Source ..........................................71

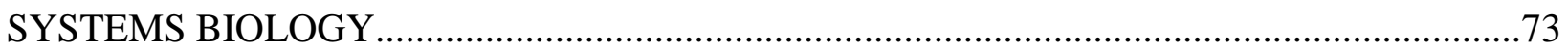

00227: Biomass Ethanol from Clostridium thermocellum:

A Systems Biology Analysis ...............................................................................73

00228: A Model System for Analyzing Whole-Body Toxicity of TICs, TIMs, and Chemical Warfare Agents ..................................................................................74

00003: Systems Biology of the Mammalian Cilium: A Cellular Organelle Essential for Human Health and Development ........................................................................76

00230: Accelerated Domestication in Populus: Harnessing the Recently Sequenced Genome for Bioenergy Crop Production...................................................77

00241: Disentangling Soil Respiration Using Genomic Techniques .....................................79

00242: Photo-Regulated Peptide-Protein Interaction Systems for Bionanotechnology Applications.

00244: Analysis of the Role of miRNAs: Profiling miRNA Expression across BXD Recombinant Inbred Mouse Strains in Support of the Mouse Collaborative Cross Program

00007: Novel Approaches for Uncovering Total Environmental Gene Expression Patterns

00020: Systemic Approaches in Recombinant Zymomonas mobilis to the Regulation of Ethanol Fermentation .85

00021: Unraveling the Regulatory and Biosynthetic Genes that Control Cellulose Production in the Model Bioenergy Crop, Populus

00033: Three-Dimensional Aberration Corrected Scanning Transmission Electron Microscopy for Studying Microbiological Systems

00040: Electricity and Biohydrogen Production via a Systems-Level Understanding of Microbial Fuel Cells

00043: Microfluidic Platform for Individual Microbe Capture, Cultivation, and Selective Release .90

00044: Methodological Development of Computer Simulation in

Molecular Biophysics

ULTRASCALE COMPUTING.

00231: Development of a Global Biogeochemistry Capability for Enhanced Climate Simulation and Earth System Modeling .........................................................93

00232: Terascale Simulation Tools for Next-Generation Nuclear Energy Systems ...............95

00235: Multiscale Modeling: Application to Hydrogen and Helium in Steels .......................96

00236: Exploring Performance Tools for Petascale Systems with Lightweight Compute Node Kernels .98

00240: Large-Scale Exploration of Protein Models for System Biology Applications.........100

00006: Exploring Reconfigurable Computing Programming Models to Accelerate High-Performance Computing (HPC) Applications 
00008: Predictive Simulation and Virtual Design of High-Speed, High-Density

Molecular and Nanoscale Sensors and Devices

00009: Storage Virtualization: An Integrated Approach to Machine-Room

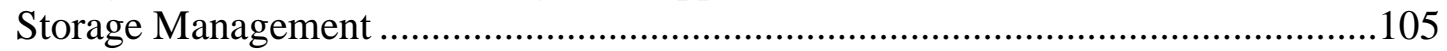

00010: Virtualized Systems Environment for Petascale Computing and Beyond ..............106

00011: Petascale Computing in Nanoscience on 100,000+ Cores ......................................108

00012: Modeling Cellular Mechanisms for Efficient Bioethanol Production through

Petascale Comparative Analysis of Biological Networks .....................................109

00014: An Evolutionary Framework for Porting Applications to Petascale Platforms ........111

00016: Development of a Global Advanced Nuclear Fuel Rod Model..............................112

00026: Waveguide Entangled Photon Sources for Quantum Information ..........................114

GENERAL

00188: Applications of Ultrafast, Ultraintense Lasers to Radioactive Ion Beam

Production and Diagnostics

00048: Theoretical and Computational Methodologies and Tools for

Second-Generation Integrated Fusion Simulation...

00246: Nanostructured Surfaces from Drawn Materials

00049: Transfer of Vertically Integrated Carbon Nanotube Arrays for Sensors

and Thermal Management

SEED MONEY FUND

BIOSCIENCES DIVISION

00396: Identification of Protein-DNA Interaction and Protein-Protein

Interaction in Single Living Cells Using Optical Nanosensors

00402: Generation of Mouse Embryonic Stem Cell Lines to Study MicroRNA

Functions through Conditional and Cell Lineage-Specific shRNA

Knockdown Approaches.

00410: A Genomic Analysis of Microbial-Mediated Metal Transformation .......................129

00420: Establishing a Targeted Mutagenesis System in Clostridium Cellulolyticum ..........130

00442: Destroying Pathogenic Bacteria Using Targeted Nanoparticles.............................132

00451: Nanostructured 3-Dimentional Electrodes for Enzyme Fuel Cells .........................133

CENTER FOR NANOPHASE MATERIALS SCIENCE

00426: Mapping Carrier Distributions and Photovoltaic Activity in

Nanophase Materials by Electrical Dissipation Microscopy...

CHEMICAL SCIENCES

00404: Development of ZnO Light Emitting Diodes Utilizing Pulse

Thermal Processing

00414: Selective Electrochemical Oxidation of Water for Treatment of

Ischemic Diseases and Other Applications. 
00424: Taming Photosynthesis Regulation through Genomics for

Direct Synthesis of Ethanol from Carbon Dioxide and Water

00425: Synthesis of Polymeric Materials for Blue-Light Emitting Diodes.........................141

00440: Probing the Molecular Interface of Cellulose and Lignin in Biomass ....................142

00453: Monte Carlo Simulation of Ion Trajectories in Ion Mobility Spectrometry .............143

00457: Turbopump Concentration of Heavy Atoms and Molecules

COMPUTATIONAL SCIENCES AND ENGINEERING DIVISION.

00397: Discrete Event-Based Simulation of Electromagnetic

Wave Propagation in Highly Cluttered Environments

00435: A Hybrid Diffusion Model Driven by Chemoattractants

COMPUTER SCIENCE AND MATHEMATICS DIVISION

00394: Smart Tunneling Barriers: A New Concept for Ferroelectric-Based

Nonvolatile Random Access Memory....

ENGINEERING SCIENCE AND TECHNOLOGY DIVISION

00406: Novel High-Resolution Micromechanical Gyroscope.

00408: Demonstration of Intra-Reactor Diagnostics for Catalytic Fuel Reformers ....

00409: Optical Monitoring of Delivery Methods for Therapeutic Agents to

Neural Tissues

00423: Live Cell Micro-Arrays for Genotypic Evaluation of Microbial Electron Transport

00436: Plasma Etching and Simulation of Electron Scattering in Nanoscale Copper

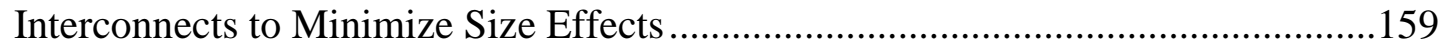

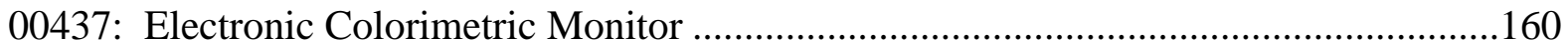

00443: A Compact Chemical-to-Hydraulic Power Source ............................................... 162

ENVIRONMENTAL SCIENCES DIVISION .............................................................. 163

00356: Alzheimer's Disease Detection via Nonlinear Analysis of EEG.............................163

00411: Determining Relative Value of Ecosystem Services ............................................164

00413: Effects of Groundwater Chemistry on the Distribution of

Soil Microorganisms in Natural Media

00419: Multivariate Statistical Analysis Technique to Locate Ecological

Observation Sites within Regional Landscapes.

00429: Development of an Advanced Surface-Enhanced Raman Spectroscopy

for the Identification and Characterization of Pollen

00430: Land-Use Dynamics and Infectious Diseases: A Systems Approach

to Defining the Causal Mechanism of Outbreak and Spread of

Eastern Equine Encephalomyelitis

00434: A Proof-of-Concept Implementation for a USA National Phenology

Network CyberInfrastructure 
00441: Quantitative Parametric Decay Simulation: A New Tool for Understanding Parasitic Nonlinear RF Power Losses in Heating Fusion Plasmas.

MATERIALS SCIENCE AND TECHNOLOGY DIVISION

00371: Diamond Films on Low-Cost Substrates for Wide-Ranging

Electronic Applications

00389: Scalable Surface-Enhanced Raman Spectroscopy (SSERS) for

Single-Molecule Detection and Characterization

00405: Quasielectrostatic Carbon Orientation (QCO) Processing for

Lithium Ion Battery Anodes and Other Applications.

00416: In Situ Monitoring of Realistic Catalyst Systems with High-Speed

Electron Microscopy Using a LaB 6 Nanowire-Based Electron Source.

00417: Microstructure and Defects in Energetic Materials and Radioactive Alloys.............180

00421: Novel, Low-Cost, High-Mn-Containing Austenitic Stainless Steels and Alloys for High-Temperature Structural Applications

00422: Laser-Interference Direct Structuring of Zirconia for Dental Materials.

00433: Organic Magnets: Phenomenological and First-Principles Approaches

00438: Can the Quantum Confinement

in Organic Spintronics?

in Organic Spintroics?

00439: In Situ Nanopatterning of Single-Crystal Multiferroics by Strain for

Terabit-Scale Data Storage ...... 186

00444: Photon-Assisted Thermoelectric Devices.

00445: Photocatalytic Conversion of $\mathrm{CO}_{2}$ : An Alternative to

Storage-Based Sequestration

00447: In Situ Studies for Ductility Improvement of Bulk Metallic Glasses.

00449: Assessment of Possible Exotic Magnetic Behavior in Anti-Perovskite Nitride...

00454: An Innovative Low/High-Temperature, Repetitive Pressure-Pulse

Apparatus for Cavitation Damage Research.

00456: High-Strength, Ductile, Crystalline-Amorphous Multilayers

00458: Deterministic Growth of Oxide Nanostructures by

Pulsed-Laser Deposition

NEUTRON SCATTERING SCIENCE DIVISION

00452: Fundamental Studies of $\mathrm{CO}_{2}$-Coal Interactions Using Novel Neutron-Scattering Techniques at Conditions Relevant to Subsurface Sequestration.

NUCLEAR SCIENCE AND TECHNOLOGY DIVISION

00412: A Novel Radio-Luminescent Glass Designed for Safe User Applications

00415: Exploring Layered Materials with Neutron and Photon Spectroscopy

to Determine the Depth and Water Content in Subsurface Layers of Planets

00418: Carbonate Thermochemical Cycle for the Production of Hydrogen

00431: Fuel Pellet Irradiation Capsules for Advanced Reactor Fuel Testing in HFIR 
00432: Generalized Perturbation Methods for Transport Computations

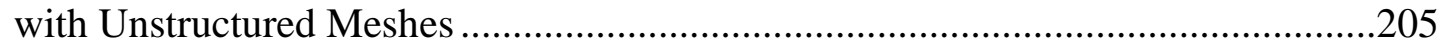

00446: Intensified Continuous Production of Biodiesel....................................................206

00448: Molecular Engineering of Core-Shell Interfaces toward Controllable Production of Brighter, Optically Tunable Quantum Dots.......................................207

00450: Development of a Hybrid Computational Phantom Model ......................................209

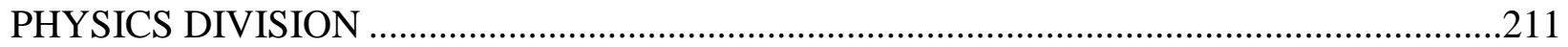

00407: Big Bang Cosmology and Online Simulation Suite ..............................................2211

00455: Computing the Electric Dipole Moment of the Neutron and the Schiff Moment of the Nucleus

AUTHOR INDEX 



\section{INTRODUCTION}

The Oak Ridge National Laboratory (ORNL) Laboratory Directed Research and Development (LDRD) program reports its status to the U.S. Department of Energy (DOE) in March of each year. The program operates under the authority of DOE Order 413.2B, "Laboratory Directed Research and Development" (April 19, 2006), which establishes DOE's requirements for the program while providing the Laboratory Director broad flexibility for program implementation. LDRD funds are obtained through a charge to all Laboratory programs.

This report includes summaries for all ORNL LDRD research activities supported during FY 2007. The associated FY 2007 ORNL LDRD Self-Assessment (ORNL/PPA-2008/2) provides financial data and an internal evaluation of the program's management process.

ORNL is a DOE multiprogram science, technology, and energy laboratory with distinctive capabilities in materials science and engineering, neutron science and technology, energy production and end-use technologies, biological and environmental science, and scientific computing. With these capabilities ORNL conducts basic and applied research and development (R\&D) to support DOE's overarching mission to advance the national, economic, and energy security of the United States and promote scientific and technological innovation in support of that mission. As a national resource, the Laboratory also applies its capabilities and skills to specific needs of other federal agencies and customers through the DOE Work for Others (WFO) program. Information about the Laboratory and its programs is available on the Internet at http:// www.ornl.gov/.

LDRD is a relatively small but vital DOE program that allows ORNL, as well as other DOE laboratories, to select a limited number of $R \& D$ projects for the purpose of

- maintaining the scientific and technical vitality of the Laboratory,

- enhancing the Laboratory's ability to address future DOE missions,

- fostering creativity and stimulating exploration of forefront science and technology,

- $\quad$ serving as a proving ground for new research, and

- $\quad$ supporting high-risk, potentially high-value R\&D.

Through LDRD the Laboratory is able to improve its distinctive capabilities and enhance its ability to conduct cutting-edge R\&D for its DOE and WFO sponsors.

To meet the LDRD objectives and fulfill the particular needs of the Laboratory, ORNL has established a program with two components: the Director's R\&D Fund and the Seed Money Fund. As outlined in Table 1, these two funds are complementary. The Director's R\&D Fund develops new capabilities in support of the Laboratory initiatives, while the Seed Money Fund is open to all innovative ideas that have the potential for enhancing the Laboratory's core scientific and technical competencies. Provision for multiple routes of access to ORNL LDRD funds 
maximizes the likelihood that novel ideas with scientific and technological merit will be recognized and supported.

\begin{tabular}{|c|c|c|}
\hline \multicolumn{3}{|c|}{ Table 1. ORNL LDRD Program } \\
\hline & Director's R\&D Fund & Seed Money Fund \\
\hline Purpose & $\begin{array}{l}\text { Address research priorities of } \\
\text { the Laboratory initiatives }\end{array}$ & $\begin{array}{l}\text { Enhance Laboratory's core } \\
\text { scientific and technical } \\
\text { disciplines }\end{array}$ \\
\hline Reviewers & $\begin{array}{l}\text { Initiative review committees } \\
\text { (IRCs) composed of senior } \\
\text { technical managers and } \\
\text { subject matter experts }\end{array}$ & $\begin{array}{l}\text { Proposal review committee } \\
\text { (PRC) composed of scientific } \\
\text { and technical staff, } \\
\text { representing the research } \\
\text { divisions, assisted by } 2-3 \\
\text { technical reviewers for each } \\
\text { proposal }\end{array}$ \\
\hline Review process & $\begin{array}{l}\text { Preliminary and full proposal } \\
\text { review, including a } \\
\text { presentation to the IRC, and } \\
\text { an annual review of progress }\end{array}$ & $\begin{array}{l}\text { Full proposal review } \\
\text { including a presentation to the } \\
\text { PRC; review of progress if } \\
\text { funding is awarded in two } \\
\text { phases. }\end{array}$ \\
\hline Review cycle & Annual & Monthly \\
\hline Project budget & Typically $\sim \$ 600,000$ & $<\$ 175,000$ \\
\hline Project duration & 24-36 months & $12-18$ months \\
\hline LDRD outlay & $\sim 80 \%$ of program & $\sim 20 \%$ of program \\
\hline
\end{tabular}

\section{DIRECTOR'S R\&D FUND}

The Director's R\&D Fund is the strategic component of the ORNL LDRD program and the key tool for addressing the R\&D needs of the Laboratory initiatives. The initiatives, which are the focus of the Laboratory Agenda, are the critical areas on which the Laboratory must concentrate if it is to be prepared to meet future DOE and national requirements for science and technology.

The success of an initiative depends to a large extent on the Laboratory's ability to identify and nurture cutting-edge science and technology on which enduring capabilities can be built. To do this, ORNL uses the resources of the Director's R\&D Fund to encourage the research staff to submit ideas aimed at addressing initiative-specific research goals. Each spring, the Deputy Director for Science and Technology issues a call for proposals. The call emphasizes specific research priorities selected by management as being critical to accomplishing the Laboratory's initiatives.

The initiatives and research priority areas for FY 2007 were as follows:

- Advanced Energy Systems: advanced technologies for energy storage, nuclear energy, alternative liquid fuels, and the electric grid;

- Advanced Materials: fundamental studies in hydrogen production, storage, and use; nanostructured materials for effective solar energy use; novel materials for 
superconductivity, solid-state lighting, and energy storage; new materials and chemical processes for nuclear energy, and new tools for characterization at the nanoscale;

- National Security: sensors and detectors for chemical, biological, and nuclear threats; mobile power sources; computational science for homeland security; materials research for security applications; and transportation security;

- Neutron Sciences: novel applications of neutron scattering; neutron physics; novel instrumentation concepts; scientific challenges of power upgrades for spallation neutron sources; and biological applications of neutron scattering;

- Systems Biology for Energy, Environment, and Health: bioenergy; detection and simulation of ecosystem response, and multigenic mammalian systems;

- Ultrascale Computing: computing science (e.g., petascale tools, performance tuning, advanced algorithms, and large data sets), science applications (e.g., scalable application software, energy systems S\&T, atomic- and molecular-scale modeling, and multiscale modeling), and knowledge management (e.g., information fusion, topological modeling from sensor data, and quantum information science).

To select the best and most strategic of the submitted ideas, the Deputy Director establishes a committee for each initiative to review the new proposals and associated ongoing projects. The committees are staffed by senior technical managers and subject matter experts, including external members from the academic community.

Proposals to the Director's R\&D Fund undergo two rounds of review. In the first round, the committees evaluate preliminary proposals and select the most promising for development into full proposals. In the second round, the committees review the new proposals and ongoing projects that are requesting second- or third-year funding. After the reviews are completed, the committees provide funding recommendations to the Deputy Director for Science and Technology, who develops an overall funding strategy and presents it for approval to the Leadership Team, ORNL's executive committee headed by the Laboratory Director. All projects selected for funding must also receive concurrence from DOE.

In FY 2007, \$21.64 million was allocated to the Director's R\&D Fund to support 77 projects, 42 of which were new starts (Table 2). About $90 \%$ of the fund's annual allocation is awarded to projects at the beginning of the fiscal year. The remainder, about $10 \%$, is held in reserve primarily to support research projects of new R\&D staff members being recruited to address strategic Laboratory needs. The levels of investment in each initiative are summarized in Fig. 1.

\begin{tabular}{llll}
\hline \multicolumn{3}{c}{ Table 2. ORNL LDRD by Fund } \\
\hline \hline & Director's R\&D Fund & Seed Money Fund \\
${ } }$ & 721.639 million & & $\$ 4.560$ million \\
Number of projects & 42 & 60 \\
Number of new starts & 35 & 35 \\
Continuing (second year of funding) & 25 \\
Average total project budget (1-3 yr) & $\$ 600,504$ & $\$ 132,645$ \\
Average project duration & 24 months & 16 months \\
\hline
\end{tabular}




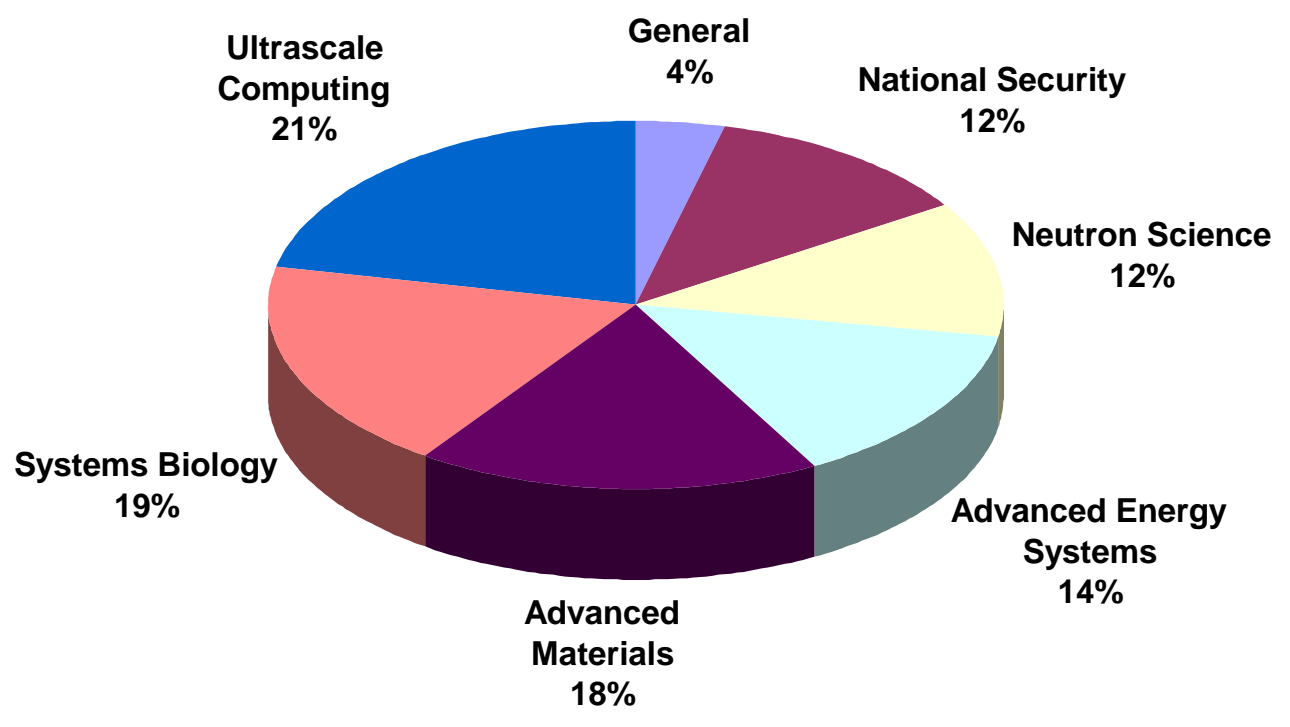

Fig. 1. Level of Director's R\&D Fund investment in the Laboratory initiatives for FY 2007.

\section{SEED MONEY FUND}

The Seed Money Fund complements the Director's R\&D Fund by providing a source of funds for innovative ideas that have the potential of enhancing the Laboratory's core scientific and technical competencies. It also provides a path for funding new approaches that fall within the distinctive capabilities of ORNL but outside the more focused research priorities of the major Laboratory initiatives. Successful Seed Money Fund projects are expected to generate new DOE programmatic or Work-for-Others sponsorship at the Laboratory.

Proposals for Seed Money Fund support are accepted directly from the Laboratory's scientific and technical staff (with management concurrence) at any time of the year. Those requesting more than $\$ 28,000$ ( $\$ 175,000$ is the maximum) are reviewed by the Proposal Review Committee (PRC), which is composed of scientific and technical staff members representing each of the Laboratory's research divisions and a member of the Office of Institutional Planning, who chairs the committee. To assist the committee, each proposal is also peer reviewed by two to three Laboratory staff members selected by the chair. Proposals requesting $\$ 28,000$ or less are reviewed by the chair, normally with the assistance of a technical reviewer. All Seed Money Fund proposals receiving a favorable recommendation are forwarded to the Deputy Director for Science and Technology for approval and require DOE concurrence. 
In FY 2007, \$4.56 million of the LDRD program was apportioned to the Seed Money Fund to support 60 projects, 35 of which were new starts (Table 2). The distribution of Seed Money Fund support by research division area is shown in Fig. 2.

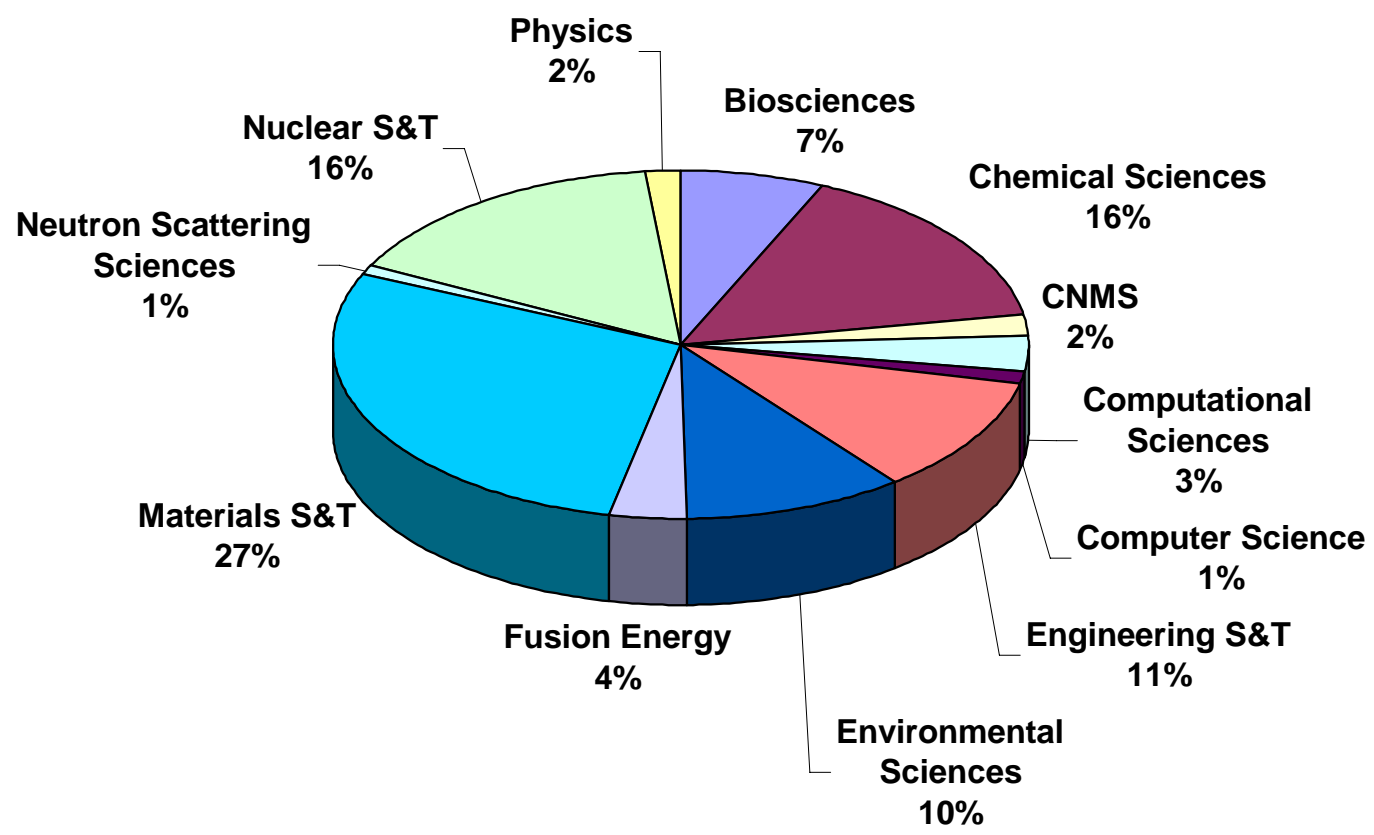

Fig. 2. Distribution of Seed Money Fund by research division for FY 2007.

\section{REPORT ORGANIZATION}

This report, which provides a summary of all projects that were active during FY 2007, is divided into eight sections: one for each of the six Laboratory Initiatives discussed above, a General Category of projects funded through the Director's R\&D Fund by the Deputy Director for Science and Technology, and the Seed Money Fund. This section is further categorized by the research division of the principal investigator. Each summary consists of three parts: project description, mission relevance, and accomplishments through the end of FY 2007. A list of publications is also provided, if the work has resulted in journal articles or conference presentations published in a proceedings. 



\section{Director's R\&D Fund}





\title{
00233: Novel Carbon Materials for Advanced Energy Storage
}

\author{
David DePaoli, Sheng Dai, Nancy Dudney, Jim Kiggans, Chengdu Liang, and Sea Park
}

\section{Project Description}

This project is focused on devising innovative materials technology for electrochemical capacitors, including nanostructured carbon for electrodes and alternative electrolytes. Electrochemical capacitors are high-power energy storage devices that have performance properties intermediate between conventional capacitors and batteries and are attractive for a wide variety of applications, both as stand-alone energy storage devices and as a complement to batteries in hybrid power systems. Currently, application of electrochemical capacitors is limited by cost and performance issues of electrode and electrolyte materials. Novel mesoporous carbon materials synthesized at ORNL exhibit large accessible surface area and controllable pore size and thus present promise for exceptional energy-storage performance. Through the experimental synthesis, characterization, and performance testing conducted in this project, we expect to develop inexpensive materials that provide greater energy and power density than currently available systems and breakthrough frequency response. We aim to develop a unique capability for tunable production of electrode materials and matching electrolytes that can deliver performance characteristics targeted for specific applications. This project, which transitions an ORNL nanoscience discovery into an energy-related nanotechnology, will provide opportunities for long-term fundamental research as well as multiple opportunities for grid, vehicle, renewable energy, and military applications.

\section{Mission Relevance}

Advanced energy storage is actively sought in a wide range of vital applications of concern to national security, including stability of the electrical grid, renewable energy production, portable power systems for military and homeland security applications, and the transition to alternative fuel-transportation systems through electric, and hybrid electric, and fuel-cell powered vehicles. This project is directly relevant to the Electricity Storage program of the DOE Office of Electricity Delivery and Energy Reliability and multiple programs of the DOE Office of Energy Efficiency and Renewable Energy, including FreedomCAR and Vehicle Technologies; Industrial Technologies Solar Energy Technologies; Hydrogen, Fuel Cells and Infrastructure Technologies; and Wind and Hydropower Technologies. In addition, there are multiple potential applications for other federal missions, including the Department of Defense.

\section{Results and Accomplishments}

Excellent results have been achieved in this project toward translating a scientific discovery for synthesis of mesoporous carbon materials through self-assembly into a practical technology for producing improved electrode materials for electrochemical capacitors. Experimental studies have demonstrated the capability to produce carbon materials with pore structures that provide high accessible surface area for ions of an electrolyte. Block copolymer surfactants have proven to be an efficient template for synthetic mesoporous carbon; the pore size can be tailored at least 
4 to $11 \mathrm{~nm}$, with a narrow size distribution. The mesoporous carbon can be prepared in several forms for electrodes with tailored properties. Testing indicates very good electrical storage performance, both in terms of high specific capacitance and frequency response. High specific capacitance is achieved for non-graphitized carbons, while high conductivity and rapid response are realized for carbons graphitized at high temperatures.

These materials are nearly ideal for investigation of electrolytes with large ions, including ionic liquids and other organic salts. Practical challenges for continued development of these materials for ultracapacitor applications include increasing pore volume for carbons with smaller pore dimensions and maintaining (or restoring) high surface areas and specific capacitance with graphitization. There is good potential for these materials to be produced at relatively low cost through scalable nanomanufacturing processes; thus, these materials show promise for continued development and optimization towards practical application.

\section{Publications}

Park, S. H., et al. 2007. "Mesoporous Carbon Materials as Electrodes for Electrochemical Double-Layer Capacitor.” 2006 MRS Fall Meeting, November 27-December 1, 2006, Boston, ME, USA.

\section{5: Multicomponent Fuel Spray Simulation Tools for Alternative Fuels}

Joanna McFarlane, Valmor de Almeida, Johney Green, Stuart Daw, Kalyan Chakravarthy, Sam Lewis, Scott Sluder, Bill Steele, Robert Wagner, and Rolf Reitz

\section{Project Description}

A new tool set was developed for modeling combustion processes that combines knowledge of chemical physical properties, chemical kinetics, and computational fluid dynamics to simulate the performance of novel alternative fuels under realistic engine operating conditions. A database of chemical information on biodiesel fuels has been established from existing literature, computational analysis, and modeling. The project integrated this chemical database with various existing software components for chemical kinetics and in-cylinder combustion (CHEMKIN III, KIVA-3-ERC) to develop simulation packages of practical value for advanced engine research. A key aspect was the reduction of the chemical mechanism so that it can be incorporated into the engineering-scale simulation, and this was accomplished using a CHEMKIN-based sensitivity analysis. The new parallelized reaction-set analysis tool (XChemKin) was shown to be linearly scalable to 32 processors. The simulations were validated for advanced combustion modes by comparison with performance and emissions results obtained at the Fuels, Engines and Emissions Research Center (FEERC) on biodiesel/diesel mixtures burned in Mercedes and Hatz diesel engines. Tools developed in this project, such as a multicomponent spray model, are applicable beyond biodiesel to evaluate engine performance with alternative fuel formulations such as oil sand fuels, heavy oils, and alcohol-based biofuels. Mechanisms and results from this project may also be used to benchmark Scientific Discovery through Advanced Computing 
(SciDAC) combustion calculations to assist in the development of supercomputing for engineering applications.

\section{Mission Relevance}

The development of alternative sources of transportation fuel is an emerging national priority aimed at reducing our dependence on foreign sources of petroleum. This project established a new research capability at ORNL of interest to two subprograms-the DOE Energy Efficiency Renewable Energy (EERE)-Fuel Technology subprogram of the Office of FreedomCAR and Vehicle Technologies (OFCVT) and Fuels Combustion of the Office of Distributed Energy (DE). The current OFCVT R\&D plan specifically references the need for a predictive tool for next-generation transportation vehicles operating in advanced combustion modes on fuels derived from biomass, oil sands, or synthetic formulations. Funds from DOE-EERE have been secured for continued collaboration with University of Wisconsin beyond the current scope of the project, and KIVA modeling will be supported by EERE. FEERC is also participating in a Work for Others project with reaction design to model the chemical kinetics of advanced combustion.

\section{Results and Accomplishments}

This report is for the end of the second year of a 3-year project on the ORNL-sponsored simulation of the combustion of biodiesel in advanced engines. Various combustion engineering aspects were investigated, including alternative fuel chemistry and physical properties, computational fluid dynamics modeling of actual engines, and comparison of simulations with state-of-the-art experimental data on alternative fuels. The work will be presented in four separate Society of Automotive Engineering publications this year and early next year. Technical gains include determination of physical properties for a variety of biodiesel components and testing of the effect of these properties on a multicomponent fuel spray model for a General Motors engine, development of geometrically accurate meshes for engines and nozzles, implementation of a multizone heat transfer model in a chemical kinetics framework, development and scaleup of automated parallel computing software for reaction mechanism reduction and implementation with the Lawrence Livermore National Laboratory (LLNL) methyl butanoate mechanism, and preparation of a reduced reaction mechansim for biodieseldiesel blends that was tested against ignition delay and $\mathrm{NO}_{\mathrm{x}}$ emission data from a Sandia National Laboratories study. Several graduate, undergraduate, and even high school students participated on the project, demonstrating the commitment of ORNL and DOE to fostering scientific education in the United States. Although the project is drawing to a close, a post-doctoral fellow will be exploring the use of neutron scattering facilities at the Spallation Neutron Source to penetrate a high-pressure spray of biodiesel and to measure the droplet size distribution in-situ.

\section{Publications}

Szybist, J. P., McFarlane, J. and Bunting, B. G. 2007. "Simulation of Biodiesel Blends HCCI in a Modified Hatz Diesel Engine," SAE 2007-01-4010. 
Charkravarthy, K, J. McFarlane, S. Daw, Y. Ra, and R. Reitz. 2007. "Differences in Physical Properties of Soy Biodiesel and n-Heptane: Implications for use of Biodiesel in Diesel Engines." SAE 2007-01-4030.

Szybist, J. P., J. McFarlane, and B. G.Bunting. 2007. "Comparison of Simulated and

Experimental Combustion of Biodiesel Blends in a Single Cylinder Diesel HCCI

Engine." SAE 2007-01-4010.

\section{4: Nanocomposite Dielectrics: New Smart Materials for Electric Power Applications and the Advanced Grid}

Isidor Sauers, Enis Tuncer, David R. (Randy) James, Mike Gouge, Parans Paranthaman, Amit Goyal, Panos Datskos, and Nickolay Lavrik

\section{Project Description}

A new class of materials, nanocomposite dielectrics, is being developed by this project that can address the needs of the advanced power grid in the twenty-first century and beyond. These materials offer the possibility of engineering the precise properties needed for each application, thus improving performance, reliability, and overall life. In general, dielectric materials for grid applications should have high dielectric strength and longevity. Depending on the particular application, dielectric materials should also exhibit high mechanical strength, thermal compatibility, high thermal conductivity, low losses, desired permittivity, or various combinations of these characteristics. Therefore, composites have been developed in which filler materials are used to effect a change in the various physical properties that are needed. The use of fillers normally results in a reduction of the dielectric strength. However, when particles are on the nanoscale (i.e., less than $100 \mathrm{~nm}$ ), the effects of the interface are significantly reduced. The program plan is to examine various polymer (or epoxy)-particle filler combinations and compare the dielectric properties of these nanocomposites with the corresponding microcomposite and with the base polymer.

\section{Mission Relevance}

Advanced materials are needed to improve the performance of electric supply and transmission equipment such as transmission lines, fault current limiters, transformers, generators, bushings, standoffs, line insulators, and capacitors. Virtually all of the grid technologies require dielectrics (i.e., electrical insulation) in one form or another to permit the continuous operation of highvoltage components and to prevent shorting to ground, leading to power failure and blackouts. Therefore, this research is relevant to the DOE Office of Electricity Delivery and Energy Reliability's (OE) Superconductivity program and the OE Gridworks program. Other programs of relevance include the Office of Naval Research for electric ship applications, the Defense Advanced Research Projects Agency cryocapacitor program, and the DOE Energy Efficiency and Renewable Energy's Freedom Car and Vehicle Technologies program for hybrid vehicles and power electronics for the development of new materials for high-energy density capacitors. 


\section{Results and Accomplishments}

During FY 2007, the project's second and final year, we made considerable progress in developing nanocomposite dielectric materials with improved dielectric and other physical properties. Toward that goal, a unique in-situ technique was developed that permitted homogeneous dispersion of nanoparticles by growing particles in solution. Several nanoparticlepolymer systems have been synthesized and tested. These include barium titanate (BTO)Araldite (epoxy), BTO-polyvinyl alcohol (PVA), and $\mathrm{TiO}_{2}$-PVA. Each of these systems (when dispersion was good) showed higher dielectric strengths than the corresponding microparticle composite. The latter three systems, in fact exhibited dielectric strengths higher than the pure polymer matrix, a result that has not been seen in ordinary composite systems. Other nanoparticles tested were $\mathrm{Nb}_{2} \mathrm{O}_{5}$ and calcium copper titanate, and other polymer matrices included poly(methyl methacrylate) (PMMA) and polyvinyl butyral resin (PVB). Improvements were observed for other dielectric and physical properties, including permittivity, mechanical strength, and thermal conductivity. These results indicate the potential for tailoring nanocomposite dielectric materials for specific properties in a variety of applications.

\section{Publications}

Tuncer, E., et al. 2007. "Electrical Properties of Epoxy Resin Based Nanocomposites." Nanotechnology. 18, 25703.

Tuncer, E., et al. 2007. "Enhancement of Dielectric Strength in Nanocomposites." Nanotechnology. 18, 325704.

\section{3: Experimental Optimization of Advanced Stellarator Confinement}

Jeffrey H. Harris

\section{Project Description}

Toroidal magnetic confinement of plasmas for fusion power production increasingly involves the exploitation of the three-dimensional character of toroidal magnetic geometry. Stellarators use external helical fields to provide stable, steady-state confinement at zero or greatly reduced plasma current, a major advantage for the achievement of practical fusion power. The most recent generation of large stellarator experiments, including the flagship Large Helical Device (LHD), still in operation in Japan, has demonstrated the viability of stellarator plasma confinement. The next generation of stellarators now being built (Wendelstein VII-X in Germany, the National Compact Stellarator Experiment [NCSX] at Princeton Plasma Physics Laboratory, and the proposed Quasi Poloidal Stellarator [QPS] at ORNL) has been designed using massive computational techniques to develop optimal magnetic configurations for maximum plasma confinement performance appropriate for reactors. These devices represent a major conceptual advance and require long construction times - they will come into full operation in 2010-2014. Because of the long lead times, it is important to use existing experiments to verify as effectively as possible the underlying physics properties that are to be 
optimized in the new devices. In this project, we have developed specific experimental scenarios using magnetic configuration variation in the Japanese LHD and (1) the magneto-hydrodynamic stability of ballooning modes, which can rupture magnetic confinement, and (2) the role of flows in regulating turbulent radial transport of particles and heat.

\section{Mission Relevance}

This work contributes directly to the Office of Fusion Energy Science (OFES) program, DOE Office of Science. The OFES program is the national basic research effort to advance plasma science, fusion science, and fusion technology — the knowledge base needed for an economically and environmentally attractive fusion energy source. This program supports the goal of the President's American Competitiveness Initiative "to demonstrate the scientific and technological feasibility of fusion energy." More broadly, fusion research also contributes to the goals of programs in Science (SC), Electricity Delivery and Energy Reliability (OE), Energy Efficiency and Renewable Energy (EE), and Nuclear Energy, Science and Technology (NE). Fusion research also provides stimulus and opportunity for industrial innovation in electromagnetics, materials, instrumentation, and control. Fusion research generally, and this project in particular, makes significant use of international collaboration on large research facilities, and thus contributes to maintaining the nation's international scientific links and profile.

\section{Results and Accomplishments}

We are collaborating internationally on the key stellarator physics issues of ballooning stability and flows and working with the teams in Japan, at the LHD facility at the National Institute for Fusion Science in Toki and the Heliotron-J experiment at Kyoto University; in Spain, with the TJ-II experiment located in CIEMAT (Centro de Investigaciones EnergŽticas, Medioambientales y Tecnologicas) in Madrid; and in Australia, with the H-1 experiment at the Australian National University (Canberra). We have used ORNL's leading stellarator design codes (VMEC,

STELLOPT, DKES, COBRA) to analyze the stability and flow behavior of different magnetic configurations, looking for key measurements that would prove/disprove physics ideas. We have determined that the LHD super dense core plasmas can become ballooning unstable in the outer plasma but are stable in the core. These stability properties may play a role in maintaining optimum plasma conditions. We have developed similar scenarios in the TJ-II, Heliotron-J, and $\mathrm{H}-1$ experiments and have developed a data mining technique on $\mathrm{H}-1$ that will allow us to search for ballooning modes in very large data sets. We have analyzed the flow patterns for LHD and TJ-II and have found that configuration variations that significantly change and even reverse the flows, and thereby alter the turbulent transport of particles and energy. As of late 2007, direct experimental measurements of ballooning activity and enhanced flows remain elusive in LHD because of diagnostic limitations. However, confinement studies in LHD show increases in effective thermal diffusivity in the outer regions of the plasma where resistive ballooning instabilities are expected, and the magnitude of these losses is comparable with those expected from theory. The 2008 LHD experimental campaign will continue these studies, using configuration variation, pellet injection, and perpendicular neutral beam injection as tools.

\section{Publications}

Ohyabu, N., et al. 2006. "Properties of the LHD Plasmas with a Large Island Super Dense Core Plasma and Island Healing." Plasma Phys. Control. Fusion 48 B383. 
Warr, G. B., et al. 2007. "Tomographic Interferometry of a Filtered High-Current Vacuum Arc Plasma," J. Appl. Phys. 101, 073302.

Dinklage, A., et al. 2007. "Physical Model Assessment of the Energy Confinement Time Scaling in Stellarators," Nucl. Fusion 47, 1265-1273.

Spong, D.A., et al. 2007. "Shear Flow Generation in Stellarators-Configurational Variations," Nucl. Fusion 47 626-633.

Harris, J. H., et al. 2007. "Stability of Super Dense Core Plasmas in LHD," Proc. EPS Conference on Plasma Physics and Fusion, July, 2007.

Lyon, J. F., et al. 2007. "Status of the Quasi-Poloidal Stellarator," Proc. EPS Conference on Plasma Physics and Fusion.

Kumar, S., B. Blackwell, and J. H. Harris. 2007. "Wire Tomography in the H-1NF Heliac for Investigation of Fine Structure of Magnetic Islands." Rev. Sci. Instrum. 78, 13501-8.

\section{4: Advanced Nuclear Fuel Examination and Testing}

Gary L. Bell, Rick Battiste, Ralph B. Dinwiddie, Charles A. Baldwin, Jeffrey L. Binder, Edgar Lara-Curzio, and Robert N. Morris

\section{Project Description}

The goals of this project are to develop (1) a high-resolution, nondestructive, thermography evaluation technique for imaging irradiated fuel and (2) an improved fuel-cladding mechanical property testing method. The first task is to develop a high-resolution infrared (IR) surface thermal analysis system for the examination of the surface and subsurface of nuclear fuel rod cladding using active and passive IR thermography. This system will be installed as a diagnostic module on the new Advanced Diagnostics and Evaluation Platform in the Irradiated Fuels Examination Laboratory (IFEL) for post irradiation examination of fuel rods; full-length light water reactor fuel rods can be examined. The second task is to develop and demonstrate full consensus standardized mechanical testing methods for nuclear fuel cladding. This includes developing techniques to determine the elevated temperature tensile hoop and tensile axial behavior of fuel cladding as a function of temperature and strain rate and producing an ASTM standard for the ORNL-developed expanded plug tensile hoop strength test.

\section{Mission Relevance}

The project will advance the state of the art in technologies for pre- and postirradiation examination and testing of nuclear fuel and cladding materials. The data generated will enable nuclear fuel rods with better fission product retention, higher plant electrical output, and improved plant reliability. This research is directly applicable to DOE's nuclear energy mission and it directly supports the goals of Next Generation Nuclear Power Plant, Generation IV and Nuclear Power 2010 programs, and the new Global Nuclear Energy Partnership program. The project will also benefit U.S. Nuclear Regulatory Commission loss of coolant accident licensing activities and spent nuclear fuel transport cask licensing activities by supporting the riskinformed/performance-based regulatory criteria obtained from a greater knowledge of the limits required to preserve fuel integrity throughout the fuel cycle. Specific examples where improved 
technical basis for regulation is needed are loss-of-coolant and reactivity transient accident initiators for in-core operation, and improved source term estimation for spent fuel in storage and under transportation.

\title{
Results and Accomplishments
}

IR Thermography: Passive thermography was selected over active thermography techniques. An internally heated fuel rod simulator containing hafnium oxide pellets with representative defects - a chip missing from a fuel pellet, a chip lodged between two pellets, and a chip lodged between a pellet and fuel cladding - was designed and fabricated. Using existing IR equipment, the rod was imaged. The defects were clearly visible in the plot of temperature as a function of position. Additional testing using the new automated linear test bed and gamma scanner in Bldg. 3525 demonstrated that IR imaging of a fuel rod when it is in the hot cell could be performed using existing equipment. Procedures for complete mapping of the clad surface surrounding fuel pellets were successfully tested and potential improvements identified.

Nuclear Fuel Clad Test Methods: To characterize the limitations associated with specimen and plug geometries, a study evaluating the effect of specimen and plug length on expanding plug hoop tensile test results was performed. Tasks completed include (1) preliminary stress analysis for design of the experimental matrix, (2) acquisition of specimen and plug materials, (3) design and fabrication of tooling for various specimen geometries, and (4) execution of experiments, including modifications as necessary. Testing of four different specimen/plug geometries was performed using copper and brass seamless tubing with dimensions similar to commercial fuel cladding. Results were used to evaluate uncertainties in measurements of force and outer diameter (OD) radial displacement. OD radial displacement and crosshead force as a function of crosshead displacement for the two materials with a specimen length of 3 OD and a plug length of 2 OD were acquired. In addition, the test infrastructure of an expanding plug hoop tensile test at elevated temperature was designed, fabricated, and demonstrated. Tests on stainless steel tubing at $400^{\circ} \mathrm{C}$ with a copper plug and $700^{\circ} \mathrm{C}$ with a nickel plug were completed. Measured load as a function of crosshead displacement data were obtained.

\section{6: A Hybrid Hydrogen Storage-Generation System Based on Bifunctional Nanostructured Photocatalysts}

\author{
Panos G. Datskos, Nickolay V. Lavrik, Barton Smith, Viviane Schwartz, Costas Tsouris, and \\ Claus Daniel
}

\section{Project Description}

Breakthrough technologies with application to energy systems can result from hydrogen production with photocatalytic splitting of water molecules. Our research relies on an innovative concept of a hydrogen generation system that utilizes unique properties of stressed nanostructures. The key idea of the proposed research is related to the fact, that certain forms of $\mathrm{TiO}_{2}$ are promising for photocatalytic splitting of water while others are efficient sorbers of molecular hydrogen. We focus our efforts on the recently discovered phenomenon of tailoring 
the energy bandgap of $\mathrm{TiO}_{2}$ via mechanical stress. Our research encompasses several model systems that incorporate $\mathrm{TiO}_{2}$ nanostructures with the goal of quantifying their properties relevant to hydrogen generation as a function of both externally applied mechanical stress and intrinsic strain. Evaluation of potential challenges associated with scaling of this concept from micro- to large-scale systems will be of our particular attention. The overall significance of the proposed concept consists in the possibility of creating hydrogen generation-storage hybrids capable of self replenishing using available solar energy. Such modules are anticipated to be used in hydrogen-based transportation and other high-energy density application.

The goal for the first 10 months of the project was to synthesize and characterize various titania phases and set up an electrochemical cell to be used in the second year for photo-electrolytic reactions.

\section{Mission Relevance}

This project is relevant to DOE's Hydrogen program and DOE's Materials Science and Technology subprogram within the DOE Office of Sciences. The present work will advance the science of stressed materials as catalysts and microfabrication. Several programs under way in the DOE Office of Sciences will benefit from the knowledge gained during this work.

\section{Results and Accomplishments}

During FY 2007, we made significant progress in synthesis of titania phases, substrate modification that promotes enhanced nanomechanical interrogation of titania phases, and comprehensive characterization of synthesized titania. The major thrust of our efforts focused on the use of the three distinctive technological strategies with potential to yield strained titania: spontaneously formed titania nanoparticles, thin film approaches, and electrochemical oxidation. We have successfully implemented several technological modifications of each of these strategies and conducted further selection of most promising candidates based on our morphological, structural, and compositional analyzes of the samples obtained. Our particular goal was to identify the processes that could induce intrinsic tensile stress or facilitate application of external strain to the resulting titania phases. To date we have (1) developed an alternative synthesis protocols to produce nanoparticles $\mathrm{TiO}_{2}$ films with anatase crystalline phase; (2) implemented thin titania films on polymeric and inorganic substrates; (3) elaborated an innovative approach to strained titania films compatible with standard microfabrication processing; (4) implemented electrochemically synthesized titania nanotubes arrays; (5) implemented high throughput laser structuring of substrates for enhanced nanomechanical interrogation of titania phases; (6) characterized surface morphology of titania polycrystalline films and structured substrates using optical and atomic force microscopies; and (7) obtained baseline optical absorbance data for various forms of titania. 


\section{7: Modular Utility-Scale Power Converters and Controllers for the Next Generation Grid}

Leon Tolbert, Madhu Chinthavali, Burak Ozpineci, Fran Li, John Kueck, and Govindarajan Muralidharan

\section{Project Description}

The development of a utility voltage power electronics module could be used in a wide variety of applications, including utilizing transmission lines to their full capacity, high voltage dc (HVDC), flexible ac transmission (FACTS), static variable (VAR) compensation, solid-state transformer, and large motor drive applications as well as for aggregation of multiple distributed energy (DE) sources. The modules developed for utility applications will have high reliability by being durable and flexible. Modules will have fault current limiting features and detection circuits such that they can limit the current through the module from external faults and can identify and isolate internal faults so the remaining modules can continue to operate with only minimal disturbance to the utility or customer. The development of a reliable, efficient, low-cost, power electronics module will be a key enabling technology for harnessing more power from distributed generation, increased use of FACTS and HVDC, and ultimately for absolute control over power flow in the grid.

\section{Mission Relevance}

The DOE Office of Electricity Delivery and Energy Reliability (OE) has interest in this research as they expand their Power Electronics and Energy Storage program. The distributed generation (DG) program is interested in how a modular power electronic interface can aid DG sources such as solar cells or microturbines in providing additional ancillary services to the grid and make them more cost effective. This project addresses one of the needs raised in ORNL Report ORNL/TM-2005/230, "Power Electronics for Distributed Energy Systems and Transmission and Distribution Applications: Assessing the Technical Needs for Utility Applications" prepared in 2005 for OE. Another DOE program that has interest in the results of this project is the Office of Fossil Energy's Solid Oxide Fuel Cell program; this office has interest in using the results of this research to investigate the possibility of aggregating fuel cells using power electronics modules.

\section{Results and Accomplishments}

An H-bridge-based modular system has been developed, which is believed to be suitable for a wide variety of utility power electronics applications such as FACTS, HVDC, static VAR compensation and distributed energy integration. The H-bridge modular system has a layered structure. A central controller via high-speed optical cable communicates with the function units. Each function unit has one or several H-bridges. Flexible system configuration can be achieved by this kind of topology. The key point here is for different applications, the only configuration work is to reconnect the H-bridge modules and set the parameters of the control software of the central controller.

A personal computer peripheral component interconnect (PCI) bus based field programmable gate array (FPGA) central controller has been designed and developed. An electric-optical 
communication interface board, which will be used to interface central and local module controllers has been designed and developed. A $5 \mathrm{~kW}$ H-bridge module with an FPGA-based modular controller has also been designed and developed. Modular functions and communications are under development and will be demonstrated with the system in FY 2008.

A method to detect and limit fault currents has been explored. A design of a gate drive that can help to protect the module from fault under load (FUL) and hard switched faults (HSF) has been developed and tested. These methods involve detecting the collector-emitter voltage across the power electronic device and modulating the gate resistance or gate voltage so as to limit the current through the power electronic device or completely turn the device off. The current limiting for a FUL type of fault is achieved by varying the gate resistance. Smaller resistance provides a low impedance path and shunts the gate-collector current, effectively limiting the peak current. Fault current limiting for HSF is achieved by varying the gate drive voltage; shortcircuit (SC) saturation current decreases with decreasing gate-emitter voltage. In FY 2008 these detection and protection circuits will be integrated with the H-bridge modules.

\title{
00038: Alternative Feedstocks for the Petrochemical Industry from No-Sulfur-Added Biomass Lignins
}

\author{
David DePaoli, Alicia Compere, William Griffith, Chaitanya Narula, and Arpad Vass
}

\section{Project Description}

This project consists of initial studies for demonstrating the feasibility of using biomass lignins to extend or replace the $3.6 \mathrm{EJ} / \mathrm{y}$ of fossil resources used as feedstocks in the production of chemicals. We aim to exploit a new capability developed by ORNL for "cleaning" lignin to convert this current high-volume waste material into aromatic feedstocks for petrochemical production. We will assess the potential for conversion of biomass lignins to polyesters and other high-volume, high-value polymers. The work includes fundamental studies of lignin properties and development of catalytic and biocatalytic processes that permit conversion of lignin into both aromatic and cyclic or aliphatic feedstocks, which are directly useful for producing a wide range of materials. Model compound systems and chemical feedstocks derived from lignin monomers will be evaluated as polymer feedstocks.

\section{Mission Relevance}

This project aims to develop transformational energy technology. Feedstock production from the lignin portion of biomass will complement the ongoing DOE initiative for cellulosic ethanol production from the nonlignin portion of biomass. The production of high-value chemicals, in addition to fuel, in a biorefinery is expected to improve the biorefinery process economics. DOE programs and federal agencies that will benefit from this research include the Biomass and Industrial Technologies programs of the DOE Office of Energy Efficiency and Renewable Energy, the Department of Agriculture, which is partnering with the DOE on the National Biomass Initiative, and the Defense Advanced Research Projects Agency, which currently has projects aimed at development of fuels from renewable sources. 


\section{Results and Accomplishments}

Efforts during the first year of the project have been primarily focused on experimental studies aimed at demonstrating the capability to convert lignin into valuable feedstocks. Excellent progress has been made toward the goals for the first year, including (a) demonstration of the capability for depolymerization of lignin into separable subunits by chemical processes, including catalytic and pyrolytic approaches; (b) demonstration of the capability for depolymerization and ring cleavage of lignin into a number of high-value feedstock chemicals by microbial and enzymatic conversions, and (c) initial process engineering and economic analyses of lignin-to-feedstock processes.

In catalytic studies, we have clearly demonstrated that the ether linkages in lignin molecules can be cleaved under mild conditions. Our pyrolysis testing of cleaned, carbohydrate-free biomass lignin showed the feasibility of producing oxygenated phenolics analogous to those used as polyester feedstocks at relatively high yield. Microbiological studies have identified consortia capable of completely degrading lignin - a notable result - and have measured the production of potentially useful metabolites, including low-molecular-weight phenolic acids in monomeric and dimeric forms. The results of these preliminary studies are provocative. In addition, initial process engineering and economic analyses of lignin-to-feedstock processes are being conducted, utilizing expert consultants, to identify compounds with the best potential as industrial intermediates as suitable targets for microbial and catalytic reactions.

\section{9: Developing a Science Base for Fuel Reprocessing Separations in the Global Nuclear Energy Partnership}

Valmor de Almeida, Richard Archibald, Joseph Birdwell, Shengting Cui, David DePaoli, Robert Jubin, Bamin Khomami, Bruce Moyer, and Costas Tsouris

\section{Project Description}

This work is aimed at developing an experimentally validated computational capability for understanding the complex processes governing the performance of solvent extraction devices used for separations in nuclear fuel reprocessing. These applications pose a grand challenge due to the combination of complicating factors in a three-dimensional, turbulent, reactive, multicomponent, multiphase/interface fluid flow system. The currently limited process simulation and scale-up capabilities provide uncertainty in the ability to select and design the separations technology for the demonstration plan of the Global Nuclear Energy Partnership (GNEP) program. We anticipate the development of science-based models for technology development and design. This project will position ORNL to address the emerging opportunity by creating an expandable process model that will be validated using an experimental capability unique to ORNL.

This project has three major thrusts, namely, a prototype experimental station, a continuum modeling and simulation effort, and molecular modeling and kinetics support. Excellent progress 
has been made in corresponding activities during this first year in (1) defining, assembling, and operating a relevant prototype system for model validation and computer-aided image processing of results; (2) establishing a mathematical model for fluid flow and transport, and deploying single-phase flow simulation; and (3) performing subscale molecular modeling and simulation.

\section{Mission Relevance}

This project is highly relevant to DOE's GNEP program and will position ORNL to play a pivotal role in developing the radiochemical separations technology required for America's nextgeneration nuclear capabilities. The radiochemical engineering science R\&D capabilities that will be developed are consistent with ORNL's mission and will provide ORNL with a basicscience-to-engineering-demonstration capability in this field. In addition to GNEP, the work will improve ORNL's position for other key national radiochemical programs, including Pu-238 production, and applications of National Nuclear Security Agency and Environmental Management.

\section{Results and Accomplishments}

In thrust area 1, we have assembled and operated a visualization setup for analysis of realistic liquid-liquid mixtures when mixed at operating conditions of centrifugal contactors. The setup is able to capture both bubbles and drops in the mixture with spatial resolution of $7 \mu \mathrm{m}$ and time resolution of $200 \mu \mathrm{s}$. A key finding from high-speed digital visualization is that air entrainment is significant in these flows. We began the development of a systematic computer-aided image analysis code for obtaining size distribution and velocities of bubbles and drops on large data sets. To our knowledge, this information has never been obtained for centrifugal contactors and will provide unique insight for the development of the fluid flow model and simulation code.

In thrust 2, we began the simulation of single-phase flow through the full geometry of a commercially available centrifugal contactor. The corresponding code is evolving towards a fully parallel code and platform to be extended to incorporate multiphases and multispecies.

In thrust 3, in collaboration with a University of Tennessee joint directed research and development (JDRD), we have begun molecular-level simulations of the extraction of aqueous uranyl nitrate based on force fields derived from existing literature. Numerous features of the system's time evolution were identified, including molecular-level structure at the liquid-liquid interface and coordination and transport of species between phases. This work provides insight into the molecular chemical transport needed for the flow simulation modeling approach of thrust 2. To our knowledge, no other research group has published these results nor performed similar simulations. 



\section{0: High-Resolution Imaging of Biological Samples in a Wet Environment}

Niels de Jonge

\section{Project Description}

We are developing a new high-resolution scanning transmission electron microscopy (STEM) technique to image individual proteins and cells in their native liquid environment with a resolution down to $1 \mathrm{~nm}$. We include the means to control and to change this environment and time-resolved measurements to observe structural changes. In our in situ STEM technique, samples in liquid are imaged in an electron microscope by using a specially designed flow cell with electron transparent windows. Biological samples, such as membrane proteins and whole cells are immobilized, grown on a window of the flow cell and maintained under live, or close to live, conditions.

\section{Mission Relevance}

This project is relevant to DOE's Biological Sciences, Chemical Sciences, and Nanoscale Science and Technology programs. In situ STEM could revolutionize imaging of biological systems on a molecular level. The project fits with DOE's goal of renewable and alternative energy sources and with the first goal of the genomics for life program, to identify and characterize the molecular machines of life on a molecular level.

This project will benefit other federal agencies as well. It will provide a new tool to image biological structures in their native environment that will benefit all programs of all agencies involved in biological sciences, medicine and drug design. This research is relevant to NIH, National Institute of General Medical Sciences, calls for the development of high-resolution probes for cellular imaging. The aim is to develop probes, thereby improving detection schemes by a factor of 10 to 100 . The ultimate goal is to develop probes that can be used to routinely achieve single-molecule sensitivity for imaging dynamic processes in living cells.

The NSF has a call on instrument development for biological research. This program supports the development of novel or substantially improved instrumentation likely to have a significant impact on the study of biological systems at any level. Proposals aimed at concept, or proof-ofconcept development for entirely novel instrumentation are encouraged.

\section{Results and Accomplishments}

During FY 2007, we have successfully tested the high-resolution and high-speed electron microscopy of whole cells (fixed E-coli bacteria). The specimen in liquid was placed in an enclosure with electron transparent windows and imaged with a STEM. The maximum resolution measured on a calibration sample containing a thin layer of gold nanoparticles was 2.5 $\mathrm{nm}$ in a $7 \mu \mathrm{m}$ thick layer of water/glycerol. Real-time images were obtained with an image 
acquisition time of 0.2 seconds and a spatial resolution of $3.5 \mathrm{~nm}$. The imaging of a wet biological specimen was tested on cells that were surface labeled with gold nanospheres or quantum dots. Individual labels could be clearly distinguished from both the top and bottoms surfaces of the cells and different signals were obtained for gold nanospheres and quantum dots.

On the basis of these results, we expect that liquid STEM presents a new route for the imaging of single molecules in whole cells and of intracellular protein function. Our results provide a unique concept for high-resolution imaging of whole cells in liquid. For fixed samples, liquid STEM presents an alternative to the current high-resolution optical methods for cellular imaging with stimulated emission depletion, or photoactivated localization microscopy. Because the speed of acquisition of those approaches cannot be increased to the order of $1 \mathrm{sec}$, they are unlikely to ever find much application in live cell imaging. In contrast, the real-time image acquisition of liquid STEM offers the potential to image cellular processes on the sub-nanometer scale in living systems.

\section{Publications}

De Jonge, N., et al. 2007. "Three-Dimensional Aberration-Corrected Scanning Transmission Electron Microscopy for Biology." In Nanotechnology in Biology and Medicine: Methods, Devices, and Applications. CRC Press, Boca Raton.

\section{4: Probing the Boundary between Imaging Microscopy and Spectroscopy: Toward the Exploration of Single Particles by Nuclear Magnetic Resonance Spectroscopy}

Edward Hagaman, Dave Geohegan, Gyula Eres, and Tony Moore

\section{Project Description}

Spectroscopic resolution on the nanoscale using nuclear magnetic resonance (NMR) is explored in this project by implementing small detector coils. Microcoils already exist that allow the successful detection of protons in $1 \mathrm{fL}$. This corresponds to a proton density on the order of $10^{12}$ protons - on the way to a truly countable number. The goal in this project is to build an NMR probe which incorporates a 30- $\mu \mathrm{m}$-diameter coil, which should allow more than an order-ofmagnitude decrease in the nuclear spin concentration. The maintenance of NMR sensitivity through better coupling of the nuclear spins to the coil and the size restrictions of the coil diameter itself conspire to allow the NMR analysis of single particles, (e.g., catalyst particles). In addition to lowering the minimum concentration of spins required for NMR detection, we will test the probe for its ability to generate intense radiofrequency field strengths for use in new solid state NMR experiments.

\section{Mission Relevance}

This work is relevant to the DOE, the National Institutes of Health $(\mathrm{NIH})$, and other agencies that fund both NMR development and applications. The microcoil NMR probe will be of interest to Chemical Sciences within Basic Energy Sciences through its program development in chemical 
imaging. In addition, the National Institute of Biomedical Imaging and Bioengineering within $\mathrm{NIH}$ supports fundamental discoveries, design, and development of technological capabilities in biomedical engineering. The microcoil probe of this work is directly relevant to many facets of selective NMR analysis in living tissues.

\title{
Results and Accomplishments
}

In FY 2006 we made significant progress in the fabrication of the microcoils. One strategy for this work, fabrication via e-beam lithography, was explored at the Cornell Nanofabrication Facility (CNF). The final microcoil dimensions present significant scale problems for lithography, and this problem interests CNF in developing the requisite lithography protocols for fabrication. Simultaneous effort at ORNL using micromanipulation techniques has succeeded in mechanically producing the needed coils. These have been incorporated into an $\mathrm{L} / \mathrm{C}$ circuit design and shown to tune correctly. In FY 2007 the ucoil/capacitor assemblies using coils with 70-130 $\mu \mathrm{m}$ diameters were incorporated into and NMR probehead and plumbed for sample delivery. The ucoil probes were evaluated using ${ }^{19} \mathrm{~F}$ NMR detection on hexafluorobenzene. These $\mu$ coil probes were tested for resolution and power handling capability. The probes are robust, able to tolerate $700 \mathrm{~W}$ pulse power and deliver radiofrequency field strengths in excess of $20 \mathrm{MHz}$ using this power level. These field amplitudes exceed our initial design target by more than a factor of two and far exceed $\mu$ coil probe performance reported in the literature.

\section{2: Synthesis and Neutron-Scattering Characterization of Ordered Self-Assembled Polymer Nanostructures and Biomembranes}

\author{
Volker S. Urban, Phillip F. Britt, Kunlun Hong, and Jimmy Mays
}

\section{Project Description}

Motions in proteins play a key role in their function. Here we establish a program developing methodological aspects of combining computer simulation with neutron scattering experiments with a view to characterising correlated dynamics in proteins relevant to bioenergy in different functional states and environments. Work is also being performed aimed at understanding the functional mechanisms of cellulase activity.

\section{Mission relevance}

Progress made through this project will place ORNL in an advantageous position to secure funding from anticipated DOE, Office of Science, BER and BES solicitations over the next several years. The results from the proposed work will, moreover, add new technological as well as scientific capabilities to ORNL, which in turn will strengthen ORNL's position as a leader in biophysical bioenergy research.

\section{Results and Accomplishments}

An extensive study has been performed of the relationship between the structure and dynamics of methyl groups in proteins. Work on peptide solvation has discovered a new driving force in 
peptide folding, associated with dehydration of exposed hydrophobic surfaces. The discovery of this new driving force has already had a major impact in the protein folding community and beyond. We have also established a computational breakthrough in the first-ever atomic-detail calculation of the vibrations in a protein crystal, and this work leads to specific predictions that will be able to be tested with instruments coming on line at the Spallation Neutron Source. Finally, work on pressure-temperature molecular dynamics has revealed the fluctuating nature of picosecond-timescale protein energy landscapes.

\section{Publications}

Schmidt, K., et al. 2007. "Reversible Tuning of a Block Copolymer Nanostructure via Electric Fields." Nat. Mater. 7, 142-145.

\section{9: Taming Electronic Spins in Conjugated Polymers for Photovoltaic and Solid-State Lighting Applications}

Jian Shen, Sheng Dai, Kunlun Hong, Bin Hu, Phillip F. Britt, and An-Ping Li

\section{Project Description}

Conjugated polymers are a new class of semiconducting materials that are revolutionizing many technologies, including energy-related applications such as solar cells, large-area flexible solidstate lighting, and lasers. The grand challenge of conjugated polymer research is to tailor the formation and dissociation of singlet and triplet excitons to maximize the efficiency of photovoltaic response and electroluminescence. We propose to meet this challenge by taking a radically new approach, namely, introducing and controlling the electronic-spin degree of freedom in the polymers. Specifically, we will dope the polymers with magnetic nanowires and use spin-polarized charge transfer, spin injection, and spin-orbital coupling to tune the singlet and triplet exciton density in conjugated polymers. Building on this concept, we plan to carry out an interdisciplinary research effort which will include (1) synthesis of conjugated polymers with electron-withdrawing side groups and surface-functionalized magnetic nanowires, (2) control of polymer/nanomaterial interface, (3) advanced characterization of optoelectronic, magnetic, and spin-dependent transport properties of the polymer/nanomaterial composites with high spatial and time resolution, and (4) fabrication of photovoltaic and light-emitting devices.

\section{Mission Relevance}

This work is relevant to the materials science and technology program within the DOE Office of Science, Basic Energy Sciences program, and supports the goal of the President's American Competitiveness Initiative to develop "chemical, biological, optical, and electronic materials breakthroughs." If successful, we expect that this project will initiate a new effort on using magnetically tunable polymer/nanomaterial composites, which is also high on DOE's Scienceto-Energy agenda. While this project has its focus on providing a fundamental understanding, we fully anticipate that it will grow into a much broader program that will impact diverse 
technologies under development at the Department of Defense (Defense Advanced Research Projects Agency, Air Force, Army) and the National Aeronautics and Space Administration.

\title{
Results and Accomplishments
}

During FY 2007, we have accomplished the following. (1) We have developed a unique experimental tool—magnetic field dependence of photocurrent—-to "visualize" the singlet and triplet processes in organic solar cells. We found that the singlet and triplet excitons experience different channels: dissociation and charge reaction in the generation of photocurrent. With this new finding, we have successfully improved photovoltaic efficiency by $60 \%$ by controlling singlet and triplet photovoltaic processes. (2) We have successfully used $\mathrm{NaYF}_{4}$ : Yb, Er upconversion nanocrystals to enhance the photovoltaic performance in conjugated polymers. Under near infra-red (NIR) excitation, the $\mathrm{NaYF}_{4}$ : Yb, Er nanocrystals emit visible light in the solutionprocessed NIR photodetectors. The emitted visible light is simultaneously absorbed by the host conjugated polymer to generate photocurrent, which indicates that our approach is applicable to fabricate NIR photodetectors and will avoid the major technical challenges associated with semiconductor quantum dots based NIR photodetectors. (3) On the chemical synthesis side, we were able to show that reasonably monodisperse nanocrystals of the ternary oxide cobalt ferrite $\left(\mathrm{CoFe}_{2} \mathrm{O}_{4}\right)$ could be synthesized in an ionic liquid in the absence of "strong" ligands. This is an essential feature because it should allow for facile place exchange reactions with ligands of choice after synthesis, opening up potential avenues to tune the solubility and assembly characteristics of magnetic nanostructures. Additionally, using a seeded growth approach, we secured preliminary evidence that a multiple injection method could be used to enlarge the colloids within the ionic liquid medium, offering a simple and reproducible means to tune the size of nanoparticles grown in an ionic liquid medium.

\section{Publications}

Sun, C., et al. 2007. "Enhancement of Quantum Efficiency of Organic Light Emitting Devices by Doping Magnetic Nanoparticles.” Appl. Phys. Lett. 90, 232110.

\section{9: Nanocrystalline/Amorphous Silicon Thin-Film Composite for Stable, High-Efficiency Photovoltaic Applications}

\author{
R. D. Ott, A. S. Sabau, R. B. Dinwiddie, J. E. Jellison, and M. J. Lance
}

\section{Project Description}

This research project involves a thermal annealing process used to introduce a nanocrystalline phase within a hydrogenated amorphous silicon phase for thin-film photovoltaics (PV). The presence of the nanocrystalline phase has shown near $100 \%$ increase in the collection efficiency of such PV devices. The ultimate goal is to introduce this nanocrystalline phase for improved PV collection efficiency while utilizing low-cost, low-temperature polymer substrates. By doing so, it may be possible to achieve the cost per peak watt of the thin-film PV system to near DOE's goal of $\$ 0.5 /$ watt. The annealing process will be performed utilizing ORNL's unique high- 
density plasma-arc lamp that has power densities approaching those of lasers but is able to process broad areas. ORNL is in a unique position because it is the only place in the world this technology can be used for research purposes.

\title{
Mission Relevance
}

Approximately $0.02 \%$ of annual energy consumption in the United States is met by PV, equating to about 0.02 quads or 0.67 gigawatt-year. Realizing the potential of this revolutionary processing capability could have a significant impact on U.S. energy production in the future. Approximately $10 \%$ of U.S. energy production could be based on PV, equating to approximately 10 quads or 335 gigawatt-years by the year 2030 based on low-cost, high-efficiency PV systems. The impact of developing such a low-cost, high-efficiency solar cell would mean the United States would have abundant, secure, and reliable domestic power and a more stable power grid in the future. Not only would DOE benefit from such a technology breakthrough, but the Department of Homeland Security would also benefit, given the fact that PV systems can reliably produce electricity at a stable cost for 30 years. Also, the ability to decentralize solar power will dramatically reduce the vulnerability of the U.S. electrical system.

\section{Results and Accomplishments}

This project has led to achieving a multimillion dollar Defense Advanced Research Projects Agency (DARPA) project focused on increased efficiency photovoltaics. ORNL has strengthened the relationship established with United Solar, who provided specimens for the project, and through the DARPA project will be able to establish a clear path forward for the manufacturing of high-efficiency nanocrystalline/amorphous silicon photovoltaics. Nanocrystalline grains on the order of $\sim 100 \mathrm{~nm}$ in size are able to be produced within the amorphous silicon thin-film photovoltaic device. This project helped the development of a process utilizing ORNL's unique high-density plasma arc-based technology to perform a solid phase crystallization process. The refined process science was established through the utilization of a process thermal model, which led to a better understanding of the operation of the highdensity plasma arc-based facility. Because of this project, a higher efficiency photovoltaic device can be produced which will better the chances of establishing photovoltaics as a mainstay in U.S. energy production.

\section{7: Nanoparticle Phase Change Materials: The Nanoscale Science Basis for Gigajoule Energy Storage}

\author{
Miroslaw S. Gruszkiewicz, Ariel A. Chialvo, David R. Cole, and Michael J. Simonson
}

\section{Project Description}

The goal of this project is to advance fundamental understanding of solid-solid transitions and interactions at the solid-fluid interface in concentrated nanoparticle dispersions as the foundation for novel, high-temperature thermal energy storage. Inorganic phase-change materials (PCM), stabilized by optimized carrier solutions that remain liquid at high temperature can provide the 
unique properties promising a dramatic increase in solar energy efficiency. Indirect experimental evidence exists for phase stability reversals driven by large differences in surface energies between nanophase polymorphs. Several questions need to be answered before the optimal nanosolid+liquid combination can be found with tunable thermal properties. Important problems include transition kinetics and reversibility over multiple cycles, stability of the nanophase in the liquid matrix, determination of the mechanism of solid-solid transformations in nanoparticles at various temperatures, and the atomic-scale source of high heat effects. The principal focus is to (1) experimentally demonstrate the principle of using high-energy inorganic solid-solid transitions in stable nanoparticles for energy storage and (2) investigate the key phenomenon of nanosolute solvation by the method of constraint molecular dynamics (MD). Success of this project will contribute to the advancement of nanoscience and enable more efficient utilization of solar thermal energy by providing a breakthrough pathway for thermal energy transfer and storage.

\section{Mission Relevance}

The DOE BES program Basic Research for Solar Energy Utilization is most likely to benefit from this project. Other target programs include Materials under Extreme Environments and Advanced Nuclear Energy Systems with subtopics related to the role of surface energy and chemistry at interfaces in confined systems. Both thermal storage and efficient characterization of nanomaterials are currently of interest to DOE Energy Efficiency and Renewable Energy. The DOE Office of Electricity Delivery and Energy Reliability initiatives (Distributed Energy program, Thermally Activated Technologies, and Recycling Thermal Energy for Combined Heat and Power Systems) could also benefit. Operation of fuel cells is part of the DoD mission. The goal of the DoD distributed energy program is to advance technologies using thermal energy storage to improve overall energy efficiency and reduce pollution. The outcome of this project may also be of interest to NASA, where it could lead to development of a simpler solution than currently used molten-salt-based energy conversion systems.

\section{Accomplishments}

Prototype PCM samples were obtained: five from a commercial source, and two from Prof. Woodfield (BYU). They include $\mathrm{TiO}_{2}$ (anatase and rutile) and $\mathrm{ZrO}_{2}$, (monoclinic and tetragonal), with average sizes between 5 and $50 \mathrm{~nm}$. The samples were characterized by SEM, XRD, and XRF under a Rapid Access proposal to the ORNL CNMS. Specific surface areas were also measured using ORNL's BET instrument. Aqueous suspensions were tested in order to assess the achievable solid loadings and stabilities. The point of zero charge and $\mathrm{pH}$ as a function of added base or acid were measured. It was found that the viscosity varied significantly with $\mathrm{pH}$, but high loadings between 20 and $46 \%$ solid by mass are practical. Six samples were sent to Prof. Randzio (Polish Academy of Sciences) for simultaneous calorimetric and volume scanning to $400^{\circ} \mathrm{C}$ using a unique transitiometer. The results for $5 \mathrm{~nm}$ anatase indicated an exothermic phase transition at $237^{\circ} \mathrm{C}$ accompanied by a clear isochoric pressure effect. Adaptation of our own HT1000 calorimeter was completed. New pressure cells were adapted and tested. The first temperature scans with $10 \mathrm{~nm}$ anatase showed a large, reproducible, endothermic effect between 270 and $240^{\circ} \mathrm{C}$ on cooling the sample starting from $300^{\circ} \mathrm{C}$. An isochoric-isothermal MD algorithm was set up for the evaluation of the potential of mean force (PMF) between two nanoparticles immersed in an aqueous solution, based on the "blue-moon" ensemble approach. We 
have focused mainly on the behavior of a pair of tetragonal "spherical" nanocrystalline particles, with a diameter of $10 \AA$ ( 45 atoms). For that purpose, we have run a series of separate simulations to cover the range of radial separation between the center of mass of the pair, toward determining the mean-force profile, and finally the corresponding PMF.

\title{
00018: Apertureless Near-Field Desorption/lonization Mass Spectrometry for Nanoscale Chemical Imaging at Atmospheric Pressure
}

\author{
Douglas E. Goeringer, Gary J. Van Berkel, Vilmos Kertesz, William B. Whitten, and \\ Robert W. Shaw
}

\section{Project Description}

Prerequisite to creating and using nanomaterials to control molecular level processes is the capability for nanoscale chemical imaging. Although scanning probe microscopy techniques enable the framework of matter to be ascertained at the atomic scale, the associated images inherently provide no information about chemical composition and molecular structure. More recently, near-field scanning optical microscopy imaging has been combined with fluorescence, Raman, and infrared systems to produce spectroscopic information at unprecedented spatial resolution $(\sim 100 \mathrm{~nm})$. In these techniques, a laser-illuminated metal probe acts as an optical antenna to produce enhancement of the radiation intensity in a localized region at the apex and is comparable in diameter to the tip (as small as $20 \mathrm{~nm}$ ). Although mass spectrometry (MS) has been enormously successful for chemical analysis and molecular structural characterization, its viability for high-resolution chemical imaging has been hampered by the limited applicability and spatial resolution of available sampling/ionization techniques. However, the same optical near-fields responsible for high-resolution optical spectroscopy also can produce nanoscale surface effects. Thus, the tip can presumably function as a near-field, desorption/ionization source for mass spectrometric chemical imaging at unprecedented nanometer-scale spatial resolution. Therefore, this effort seeks to demonstrate proof of principle for near-field desorption/ionization MS under atmospheric pressure conditions and to apply the process to nanoscale chemical imaging.

\section{Mission Relevance}

Revolutionary technologies are needed to address this country's continually increasing energy demand. Among the most promising areas for development is creation of nanoscale materials to control molecular processes in areas such as fuel cells, catalysis, and photovoltaic cells. However, the full promise of nanoscience will not be realized without the capability for detailed investigation of chemical processes occurring on individual nanoparticles or at isolated active sites. The understanding garnered from such nanometer-scale chemical characterization will guide researchers in the DOE Office of Science in materials and nanoscale science by tailoring and optimizing nanosystems for control of particular reaction paths and kinetics. Direct mass spectrometry imaging with subcellular spatial resolution would likely be of interest to the 
National Institutes of Health for investigation of the molecular and biochemical nature of soft matter, such as plant and biological tissue. In addition, the potential analytical capabilities of near-field desorption/ionization MS might be valuable to Defense Advanced Research Projects Agency (DARPA) in regard to classified measurement problems requiring the capability for nanoscale chemical imaging.

\title{
Results and Accomplishments
}

Results obtained during this fiscal year conclusively demonstrated near-field enhancement and confinement of optical radiation resulting from laser illumination of a sharp probe tip, two effects crucial to achieving the eventual goal of nanoscale chemical imaging using mass spectrometry. In the proof-of-principle experiments, optical radiation from a pulsed laser was directed toward the probe tip/sample surface region of a scanning probe microscope at an oblique angle of $\sim 30^{\circ}$. The optical beam then was focused to a diffraction-limited, $\sim 5$ micrometer diameter spot. After exposure to a single 0.2 microjoule pulse of $532 \mathrm{~nm}$ photons (with the laser beam polarized parallel to the vertical axis of the tip), the resulting desorption crater in a thinfilm of $\mathrm{In}_{2} \mathrm{O}_{3} / \mathrm{SnO}_{2}$ (ITO) was approximately $160 \mathrm{~nm}$ in diameter and $3 \mathrm{~nm}$ deep. Similar experiments with the beam polarization rotated $90^{\circ}$ showed no discernable surface effects. Additionally, when the tip was retracted, 5 microjoules were required to reach the desorption threshold. The diameter of the crater in that case was $\sim 6 \mu \mathrm{m}$ (i.e., comparable to the diameter of the focused laser beam). Of further note is that the tip-enhancement phenomenon was seen using a silicon tip, which to our knowledge has never been observed before. Additional results using a standard (i.e., without a probe tip) atmospheric pressure/laser desorption ionization mass spectrometer (AP/LDI-MS) illustrated that intact, gas-phase molecular ions of a selected benchmark molecule, nickel etioporphyrin III, could be generated without codeposition of a radiation-absorbing matrix, which is another criterion important for chemical imaging of realworld samples.

\section{7: Fundamental Mechanisms of Self-Assembly of Ordered Nanostructures in Heterogeneous Ceramic Materials}

\author{
G. Malcolm Stocks, Amit Goyal, Jianxin Zhong, and Yanfei Gao
}

\section{Project Description}

Successful fabrication of ordered arrays of nanostructures of one multicomponent ceramic material embedded in another multicomponent ceramic matrix provides enormous application opportunities in high temperature superconductors, multiferroics, and many other materials systems with high potential for technological applications. Motivated from the recent demonstration of nanoscale selfassembly of $\mathrm{BaZrO}_{3}$ nanodots in a $\mathrm{YBa}_{2} \mathrm{Cu}_{3} \mathrm{O}_{7-\delta}$ superconducting ceramic thin film using pulsed laser deposition, this project examines the simultaneous, phase separation and ordering (SPSO) of embedded nanostructures in heterogeneous ceramic materials, via synergistic efforts in fundamental theoretical formulations, simulations, and experiments. The project scope includes the development of a continuum-based, non-equilibrium thermodynamics model as well as atomistic models to study the growth kinetics, complemented 
by experimental fabrication and validation of novel self-assembled nanostructures. The outcome of this research provides guidance in the development of material selection and controllable nanostructural evolution for fabrication of novel materials.

\section{Mission Relevance}

This project is directly relevant to DOE Office of Basic Energy Sciences programs in hightemperature superconductivity, advanced nanostructured materials, computational science, and material theory. At ORNL this work is particularly relevant to stated goals of the Center for Nanophase Materials Sciences (CNMS) and the Leadership Computing Facility (LCF). For nonsuperconductivity applications, we can directly extend our work to incorporation of oxide nanodots/nanorods within a ceramic matrix for energy applications such as energy-efficient solid-state lighting (SSL) and photovoltaic cells, which are of great interests to DOE Basic Energy Sciences and Energy Efficiency Renewable Energy, Department of Defense, National Science Foundation, and many high-technology companies.

\section{Results and Accomplishments}

During FY 2007, we have focused on the study of (1) the mechanism for the process of simultaneous film growth and nanostructural self assembly, (2) the dependence of nanostructural evolution on the growth parameters, and (3) experimental correlation of nanostructures and functional properties. Particularly, synergistic experimental and theoretical effort has led to significant advances toward a theoretical understanding and experimental control of SPSO process in our prototype BZO-YBCO high-temperature-superconducting (HTS) system. Experimentally, both simultaneous deposition of BZO and REBCO (rare earth elements) from a single target, as well as sequential multilayer deposition from different targets of BZO and REBCO, have revealed a number of intriguing nanostructured thin films with superior superconductivity properties. Concomitant theoretical work has found that the nanoscale BZO patterns form to minimize the combined free energy of mixing, phase boundary, and elastic interaction, which originates from the lattice mismatch. We suggest that during film growth, the earlier pattern will guide the subsequent self-assembly process, thus attaining desirable vertical alignment and replication of BZO nanodots. A phase-field model has been developed to study the kinetics of this ordering process. Our theoretical and simulation work enabled an experimental control of size, spacing, and ordering of the embedded nanostructures via changing growth conditions, such as the deposition rate, the growth temperature, and the BZO volume fraction. 


\section{9: Imaging Energy Materials in Operandi with Atomic Resolution Scanning-Transmission Electron Microscopy}

Albina Y. Borisevich, Stephen J. Pennycook, Andrew R. Lupini, Gabriel Veith, and Niels de Jonge

\section{Project Description}

In this project, we are aiming to provide an atomic-level characterization of dynamic structure evolution and electron transfer processes in materials which are central to the energy mission of DOE. This goal will be achieved by conducting atomic-resolution Scanning Transmission Electron Microscopy (STEM) and electron energy loss spectroscopy (EELS) within local environmental and/or electrical cells. Composition and pressure of reactive gases, temperature, and bias will be varied across the sample to induce controllable changes in chemical composition and oxidation states. Here, we propose to study charge collection in solar cells via electron beam-induced current (EBIC) to observe interface evolution under bias and to study heterogeneous catalysis in action. This project will provide crucial information about processes in advanced catalysts, solar cells, and fuel cells, which are currently invisible to atomic-scale study. We will observe structural changes and electron transfer processes in materials as they happen in their native environment. Mechanisms and limitations of energy conversion processes in catalysts, solar cells, and fuel cells will be revealed. This program is ideally based in MSTD's Electron Microscopy group, with access to four aberration-corrected STEMs; it will establish a foundation for future programs and/or user facilities in these critical areas of advanced energy materials.

\section{Mission Relevance}

This project will provide crucial information about processes in advanced catalysts, solar cells, and fuel cells, which are critical to the energy mission of DOE. Mechanisms and limitations of crucial energy conversion processes will be revealed, enabling more efficient processes and materials to be developed, for the benefit of DOE and the nation. This program will extend ORNL's leadership in aberration-corrected STEM into the field of in situ imaging. The subject matter has direct relevance to many energy materials systems of importance to the DOE Office of Basic Energy Sciences. Depending on the progress in specific directions, the developed techniques of real device characterization will allow us access to the more applied branches of the DOE and external funding from the Office of Naval Research, Army Research Office, and other sources. The fundamental scientific advances will also be useful to all federal agencies undertaking basic scientific research in materials, including the National Science Foundation, National Aeronautics and Space Administration, and Department of Defense.

\section{Results and Accomplishments}

In preparation for the in situ studies, structure-property relationships were investigated in a set of promising materials systems. In the novel composite photovoltaics based on a mesoporous $\mathrm{TiO}_{2}$ matrix with deposited CdSe nanocrystals, nanocrystal adhesion to the matrix was evaluated as a function of matrix crystallinity, nanocrystal composition, and preparation conditions. The approaches to sample preparation compatible with electrical measurements for these complex 
structures have been tested. Interplay between structure and catalytic activity was examined in prospective hydrocarbon combustion catalysts $\mathrm{SnO}_{2} / \gamma \mathrm{Al}_{2} \mathrm{O}_{3}$ and $\mathrm{Pt} / \mathrm{SnO}_{2} / \gamma-\mathrm{Al}_{2} \mathrm{O}_{3}$. Preliminary results have also been obtained for a series of alkali promoted $\mathrm{Pt} / \gamma-\mathrm{Al}_{2} \mathrm{O}_{3}$ catalysts prepared using traditional impregnation techniques. Significant progress has been made in the hardware development. VG Microscopes HB601, the chosen platform for this project, was recently upgraded by incorporation of a Nion aberration corrector and efficient EELS system, making it a modern machine with $0.1 \mathrm{~nm}$ spatial resolution and $0.35 \mathrm{eV}$ energy resolution. The design of the EBIC-enabled sample holder for the VG601 is being finalized. Sample architecture for EBIC measurements has been developed. We have also evaluated the use of a commercially available sample holder manufactured by Nanofactory Instruments. It will be very useful for this program by providing the possibility for applying electrical bias locally with a scanning probe microscopy (SPM) tip.

\section{0: Antiferroelectric Thin-Film Capacitors for Ultrafast High-Power Energy Storage}

Ho Nyung Lee, Matthew F. Chisholm, David Singh, and Darrell G. Schlom

\section{Project Description}

Complex oxide antiferroelectrics (AFEs), such as (doped-) $\mathrm{PbZrO}_{3}$, offer the potential for highenergy storage densities due to the characteristic increase of dielectric constant and electric polarization with applied field; this behavior is opposite to that seen in relaxor ferroelectric (FE) capacitors. Due to ultrafast response, they have great promise for the potential application in microelectronic devices. The delicate interplay between long-range electrostatic forces and local electric/elastic configurations plays a critical role and has a real possibility to artificially generate the AFE phase from otherwise FE material. In this project, therefore, we search for novel properties and unobserved behaviors of complex oxide perovskite thin films and heterostructures that have an AFE-to-FE transition under the applied electric field. Piezoelectric strain induced in epitaxial FEs and functionally cross-coupled nanoscale heterostructures are also being investigated under extreme conditions, such as at high electric fields and with nano-sized capacitors, in order to explore unprecedented behaviors and/or properties. The state-of-the-art capabilities of thin film growth by pulsed laser deposition and "all electron, general potential linearized augmented planewave method" for first-principles theory as well as Z-contrast scanning transmission electron microscopy for imaging and chemical analyses are employed. With these tools, this project will contribute to discovering novel materials and functionalities that provide revolutionary advances in high-power density storage.

\section{Mission Relevance}

This work gives ORNL an opportunity for world leadership in understanding materials for highenergy and power-density storage. Because the quest for new energy storage materials is central to a broad range of research programs, the prototype AFEs developed in this project undoubtedly will also open doors to follow-on funding in both directly (e.g., current and future Basic Energy Sciences calls, as well as Energy Efficiency and Renewable Energy interest in vehicular energy 
storage) and indirectly related areas (i.e., electronic materials, sensors, and resistive switching/data storage) that currently are supported by several sponsors.

\title{
Results and Accomplishments
}

The FY 2007 (the first year of the project) focus was on two areas in which we made significant progress: (1) We explored the competing instability between the AFE and FE to find materials that in thin film form have an AFE-to-FE transition under the application of electric field, with as large a ferroelectric polarization and as high a switching field as practical. By first-principles calculations, we found that the orthorhombic phase of $\mathrm{PbZrO}_{3}$ is more stable than the rhombohedral one by $2.3 \mathrm{mRy}$ per formula unit. The sound velocity calculations show that, depending on the orientation, either phase can be effectively stiff. This suggests we can use control via strain (i.e., via heteroepitaxy in thin films) to favor one phase over the other. The calculated polarization of the rhombohedral ferroelectric phase is very large, $55 \mu \mathrm{C} / \mathrm{cm}^{2}$; (2) We probed the extremely high piezoelectric strain and dielectric strength, and ultra-fast piezoelectric response of $\mathrm{Pb}(\mathrm{Zr}, \mathrm{Ti}) \mathrm{O}_{3}(\mathrm{PZT})$ ultra-thin thin films by time-resolved hard X-ray microdiffraction. We found that the PZT film responds piezoelectrically within a few nanoseconds. This provides important evidence that a ferroelectric capacitor with such a fast response time can charge and discharge at a similar time scale. Moreover, we also found that extremely large piezoelectric strain can be induced in PZT films (reaching values as large as $2.7 \%$, about six times larger than that of PMN-PT ceramics) by applying very short pulses (a few nanoseconds) up to $5 \mathrm{MV} / \mathrm{cm}$. Therefore, this implies that when PZT epitaxial films are used in capacitors, not only a high-power energy density but also a strong piezoelectric response useful for many applications, such as medical imaging, can be readily achieved.

\section{1: Energy Flow and Conversion on the Molecular Level: A View of Molecular Photoelectromechanical Machines}

\author{
Sergei V. Kalinin, G.M. Brown, I. Ivanov, P. Kisari, S. Jesse, and K. Seal
}

\section{Project Description}

The convolution of societal, economic, and political factors in the recent years has propelled global warming to the forefront of public awareness, stimulating the search for renewable green energy sources and efficient energy utilization. Organic molecular systems suggest a possible solution, instigating research efforts to develop novel materials and device structures for organic photovoltaic systems (OPV) and organic light emitting devices (OLED). Often, these systems demonstrate extremely high (50-90\%) quantum yields in molecular form, which drops to 1-5\% in device structures. A crucial task in maximizing the efficiency of molecular systems is developing the capability to measure, on the nanometer and ultimately molecular levels, the individual processes involved in energy conversion, including (1) photon absorption and exciton formation, (2) exciton diffusion and separation, and (3) charge transport, and effects of molecular organization, surrounding medium and substrate on these processes. This project has the objective of separating these systems into individual functional components that are assembled in 2D molecular layers. These systems are amenable both to scanning probe imaging and 
macroscopic spectroscopic measurements. This will allow the individual processes involved in solar energy conversion to be addressed separately. Observing and understanding these steps will allow for the design of materials with increased efficiencies and extended life times of OPV and OLED systems. This project will establish the foundation for the new area of molecular electromechanical machines_a means for actuation and manipulation on molecular level.

\section{Mission Relevance}

Understanding fundamental mechanisms of energy conversion between light, electrical, and chemical in organic and bioinspired systems is one of the highest priorities for the the DOE Office of Basic Energy Sciences. Optimization of energy efficiency in OPV and OLED devices is a priority for DOE Energy Efficiency and Renewable Energy. This proposal directly addresses these missions, by (a) creating the model systems that allow nanometer and molecular-scale studies of energy conversion phenomena in molecular systems and assemblies, (b) studying their self-assembly as a route for self-repairing devices, and (c) studying electroluminescent and photovoltaic phenomena on the nanoscale in both molecular systems (BES) and realistic multicomponent devices (EERE). Beyond DOE, this project is directly relevant to Defense Advanced Research Projects Agency mission (artificial vision, molecular optoelectronics) and optical data storage. Electrical scanning probe microscopy in liquid environment and shielded probes developed here are an enabling component for probing cellular and biomolecular systems, relevant to the National Institutes of Health.

\section{Results and Accomplishments}

The primary FY 2007 effort focused on the search for molecular platforms to carry OPV and OLED functionality and development of experimental methods for macroscopic and nanoscale probing of photovoltaic and electroluminescent phenomena. We have explored the synthesis of the dialkylthiole calix[4] arene derivatives and studied the self-assembly, demonstrating the formation of disordered vertical and stripe phases at thermodynamic equilibrium on $\mathrm{Au}(111)$. To assess the evolution of the chemical and electronic states in prototype devices, we have designed and tested two electroluminescent cells with a three-electrode configuration for a fluorometer and Raman spectrometer with precise control of the potential of the working electrode. We developed and tested an SPM system for nanoscale probing of photovoltaic and electroluminescent processes in ambient and liquid environments that allows (a) direct and evanescent wave illumination by the white light, (b) DC and AC (up to $2 \mathrm{MHz}$ ) laser illumination in the direct or evanescent modes, (c) high-voltage (0 to $50 \mathrm{~V})$ tip biasing, and (d) electroluminescent detection with single photon sensitivity in the 300-800 $\mathrm{nm}$ range. To avoid the limitations imposed by the ambient degradation inherent to virtually all photoactive polymers, we have suggested studies in liquid, emulating devices such as Graetzel cells. This direction led us to a set of spectacular results, including (a) determination of the localization of the electric field in the tip-surface junction for different solvent polarities using model ferroelectric systems, (b) direct measurement of the electric fields using fabricated microelectrochemical cells, (c) development (with P. Rack) of insulated probes for liquid imaging, and (d) mathematical description of cantilever dynamics under electrical excitation in the presence of hydrodynamic loading and finite, tip-surface interactions. 


\title{
00032: Nanostructured Thermoelectrics for Power Generation: Smaller Is Cooler
}

\author{
Rongying Jin, Ho Nyung Lee, Sheng Dai, Zhixian Zhou, Gyula Eres, and Brian C. Sales
}

\section{Project Description}

This project focuses on synthesizing novel thermoelectric materials (Co-based layered oxides) with two-dimensional (2D) and one-dimensional (1D) nanostructures and developing state-ofthe-art techniques to measure their thermopower and thermal and electrical conductivities, so that the efficiency of these nanostructured materials can be accurately determined. While thermoelectric properties of both 2D thin films $\left(\mathrm{Bi}_{2} \mathrm{Sr}_{2} \mathrm{Co}_{2} \mathrm{O}_{9}\right)$ and $1 \mathrm{D}$ nanostructures $\left(\mathrm{LiCoO}_{2}\right.$ nanowires and $\mathrm{Ca}_{3} \mathrm{Co}_{4} \mathrm{O}_{9}$ nanotubes) are being investigated, detailed studies of property anisotropy of bulk $\mathrm{Na}_{\mathrm{x}} \mathrm{CoO}_{2}$ and $\mathrm{Ca}_{3} \mathrm{Co}_{4} \mathrm{O}_{9}$ indicate that the unexpected good thermoelectric properties are related to their unique crystalline structures. Our goal for the second year of this project is to build on the foundation of capabilities and materials established during our first year. We will systematically tune the growth conditions of the proposed nanostructured materials and investigate their thermoelectric quantities. This will include investigating how to improve our growth techniques for producing high-efficiency thermoelectric materials.

\section{Mission Relevance}

The proposed capability for thermoelectric property measurements on 1D and 2D nanomaterials will serve basic research needs for nanoscience and nanotechnology development, particularly for power generation. This research will benefit the DOE Basic Energy Sciences program for solar energy utilization and energy efficiency (in response to this, a full proposal has been submitted) and DOE-EERE program for FreedomCAR and Vehicle Technologies. This project will also benefit other federal agencies such as the DARPA Direct Thermal to Electric Conversion program and NASA Power and Propulsion programs.

\section{Results and Accomplishments}

In the first year of the project, we made significant progress in five aspects. (1) Thermoelectric and structural properties of single crystalline $\mathrm{Na}_{\mathrm{x}} \mathrm{CoO}_{2}$ and $\mathrm{Ca}_{3} \mathrm{Co}_{4} \mathrm{O}_{9}$ were studied, aimed at understanding the mechanism for having high thermopower with low thermal conductivity and electrical resistivity. (2) High-quality $\mathrm{Bi}_{2} \mathrm{Sr}_{2} \mathrm{Co}_{2} \mathrm{O}_{x}$ epitaxial thin films were successfully grown using pulsed laser deposition. Preliminary measurements reveal that the electrical conductivity is enhanced compared to its bulk form, while it retains high thermopower. Although the thermal conductivity is yet to be measured, good electrical conduction is desirable for thermoelectric performance. (3) Several nano thermoelectric materials with a 1D character were grown, including $\mathrm{LiCoO}_{2}$ nanowires with diameters in the range of 7 to $\sim 9 \mathrm{~nm}$, and $\mathrm{Ca}_{3} \mathrm{Co}_{4} \mathrm{O}_{9}$ nanotubes with diameters smaller than $500 \mathrm{~nm}$. (4) The effect of zero-dimensional nanostructures (Ag nanoparticles with diameters less than $10 \mathrm{~nm}$ ) on the thermoelectric properties of $\mathrm{Ca}_{3} \mathrm{Co}_{4} \mathrm{O}_{9}$ powders was explored. (5) Nano devices for measuring thermoelectric properties of an individual $\mathrm{TiS}_{2}$ nanobelt were fabricated. 


\section{1: Smart Materials toward a New Paradigm of Super-Efficient Separations Using only Energy Input: Conformational Switching Based on Magnetic Nanoparticles}

Bruce A. Moyer, Peter V. Bonnesen, Radu Custelcean, Lætitia H. Delmau, Adam J. Rondinone, Frederick V. Sloop, Jr., Volker S. Urban, and Jonathan Woodward

\section{Project Description}

This work will introduce a revolutionary new class of molecular switches based on the hypothesis of magnetically induced conformational change. This concept will be exploited to bring about a dramatic increase in the efficiency of separations, a critical energy-technology area. If extractants could be switched on and off using only energy input instead of chemically based cycling, binding-release cycles would be so efficient that huge concentration factors would be achievable using small equipment and creating no secondary waste. That is just what is needed for transforming chemical separations in the nuclear fuel cycle and in many other applications. Specifically, we aim to tether a selective metal ion receptor between an anchor substrate and a magnetic nanoparticle (NP) such that the stretching effect caused by the force acting upon the NP in a magnetic field leads to an unfavorable conformational change in the receptor, resulting in the release of its bound metal ion. The prototype switchable system will employ $\mathrm{CoFe}_{2} \mathrm{O}_{4}$ NPs tethered to selected extractive agents, such as polyethylene glycols (PEGs), crown ethers, or calixarenes, which are additionally anchored to a solid surface such as quartz or silicon. The synthesized materials are being characterized by SEM, TEM, X-ray and neutron scattering and reflectometry, FTIR reflectance-absorption spectrometry, ellipsometry, and tracer distribution measurements.

\section{Mission Relevance}

This work will benefit the DOE Office of Science Basic Energy Sciences program; however, spin-offs can be expected in separations, decontamination, environmental cleanup, and detection, benefiting programs in the DOE Office of Nuclear Energy, Office of Environmental Management, and National Nuclear Security Administration. Other likely beneficiaries of this technology are DoD programs (DARPA and SERDP) and DHS, particularly in developing technologies for radionuclide decontamination or detection. An important follow-on proposal (still pending) has been submitted to the BES Advanced Nuclear Energy Systems (ANES) program in FY 2007.

\section{Results and Accomplishments}

We have (1) successfully prepared cobalt ferrite nanoparticles and coated them with a reactive layer of isocyanate-terminated propylsilane groups; (2) coated silicon wafers with amineterminated PEG molecules; and (3) demonstrated the attachment of NPs to the PEG-coated silicon wafers. Initial batches of 3-5 nm cobalt ferrite NPs were prepared and covalently coated with reactive isocyanate groups for further derivatization. Transmission electron microscopy with elemental analysis showed well-dispersed NPs with clear indication of silicon on the surface. Preparation of larger nanoparticles using ionic liquids as the reaction medium was initiated with promising results. Silicon wafers with a $\mathrm{SiO}_{2}$ polished coating on one side were 
employed as substrates for surface modification performed in three steps: (1) functionalization of the surfaces with an isocyanate, (2) attachment of PEG molecules to the isocyanate groups, and (3) attachment of the cobalt ferrite NPs to the surface-bound PEG. Wafers were first functionalized with $\mathrm{Si}-\left(\mathrm{CH}_{2}\right)_{3}-\mathrm{NCO}$ moieties through $\mathrm{Si}-\mathrm{O}-\mathrm{Si}$ linkages, and X-ray reflectometry detected the organic silane monolayer. Attachment of PEG was achieved by soaking the coated wafers in a solution containing monoprotected diamino PEG under dry conditions. X-ray reflectometry showed that the PEG layer was $2.2 \pm 1.4 \mathrm{~nm}$ thick, corresponding to each molecule occupying $3.4 \mathrm{~nm}^{2}$ of surface area. This implies that the PEG coating so far formed is not a dense brush but rather a collapsed coiled or entangled film. The reactive NPs described above were tethered to the amine-terminated PEG layer on the wafers by soaking the wafers in a dilute dispersion of the NPs in chloroform. The X-ray reflectivity pattern indicated the presence of 4 $\mathrm{nm}$ NPs on the surface of the wafer. The particles were observed by electron microscopy to be sparsely populated over the wafer surface. Ellipsometry measurements generally confirmed the $\mathrm{X}$-ray reflectometry results. In summary, progress has led to the critical demonstration of attachment of NPs on flat surfaces. Current efforts are concentrated on increasing NP size and density of coverage on the surfaces toward the goal of magnetic alignment and testing of the proposed concept of magnetically induced conformational change.

\title{
00042: Design of Point Defect Trapping Centers in Nanostructured Nickel for Advanced Nuclear Applications
}

\author{
D. T. Hoelzer, C-L. Fu, M .K. Miller, J. Bentley, and X. L. Wang,
}

\section{Project Description}

The purpose of this proposal is to achieve the scientific knowledge for designing a high number density of nanoscale clusters, or nanoclusters, in a prototype face-centered cubic (fcc) metal based on nickel (Ni). Nickel-based superalloys have immense strategic importance in hightemperature, non-nuclear applications and corrosive environments, but do not perform well in neutron-irradiation environments. We propose that nanoclusters in Ni will provide significant high-temperature strengthening and also greatly improve the tolerance of Ni-to-neutron irradiation damage by functioning as trapping centers for neutron-irradiation-induced point defects and helium, making Ni-based alloys more desirable for advanced nuclear reactor applications. We will use an integrated theoretical and experimental approach for designing the nanoclusters in fcc Ni. Specifically, we will use quantum physics-based calculations of defect structures in $\mathrm{Ni}$, including the determination of optimal structures and binding energies of nanoclusters in Ni matrix. This will involve the calculation of the interaction between interstitials and vacancies and the solubility of interstitials in Ni. The effect of solute atom additions on the binding energy of the interstitials will then be calculated. The binding energy of interstitials will be compared with the formation energies of stable alloy phases with the solute atoms as constituents. The goal of these calculations will be to determine the most likely vacancyinterstitial (V-I) pair and solute atoms that will interact with the V-I pairs to form energetically stable clusters. The experimental study will consist of mechanical alloying and will use advanced characterization of ball-milled powders to validate the theoretical models. 


\section{Mission Relevance}

This project is relevant to DOE initiatives on Advanced Materials and Advanced Energy Systems (AES). Advanced nuclear reactors (ANR) are one of the few energy sources that can meet the steadily increasing global demand in new electric generation capacity while simultaneously reducing harmful emissions of greenhouse gases and the dependence of the U.S. economy on foreign oil from the Middle East. However, the ANR being considered by the Generation IV (GEN-IV) and Global Nuclear Energy Partnership (GNEP) programs will require significant advancements in the performance of existing structural materials. These programs have interest in Ni-based superalloys, but their poor performance with neutron irradiation damage limits their consideration for use. The successful completion of this project may lead to a more radiation-tolerant, Ni-based alloy that could have a significant impact on future decisions by the GEN-IV and GNEP programs.

\section{Accomplishments}

The theoretical modeling and experimental results of this study demonstrate that it is possible to form oxygen-rich nanoclusters in fcc $\mathrm{Ni}$. The theoretical modeling showed that oxygen $(\mathrm{O})$ exhibits the strongest vacancy $(\mathrm{V})$ attraction compared to carbon and nitrogen and the vacancy mechanism was very important for lowering the binding energy of $\mathrm{O}$ in $\mathrm{Ni}$ and increasing the $\mathrm{O}$ solubility. Further lowering of the $\mathrm{O}-\mathrm{V}$ binding energy is achieved by the strong bonding between a $\mathrm{V}$ and titanium (or zirconium). It was found that $\mathrm{O}-\mathrm{V}$ clusters containing titanium (Ti) and zirconium $(\mathrm{Zr})$ are more stable than similar $\mathrm{O}$ clusters containing no vacancies. However, the binding energy of $\mathrm{O}$ in the presence of $\mathrm{Ti}$ is still higher than the heat of formation of $\mathrm{TiO}_{2}$, implying that further alloying additions (with higher affinity for $\mathrm{O}$ than $\mathrm{Ti}$ ) are necessary to promote nucleating nanoclusters in $\mathrm{Ni}$. The $\mathrm{Zr}$ clusters show a similar trend to Ti clusters, except that $\mathrm{Zr}$ clusters are more energetically unstable relative to the heat of formation of $\mathrm{ZrO}_{2}$. To validate these models, the experimental study focused on forming oxygenrich nanoclusters in the $\mathrm{Ni}-\mathrm{Zr}-\mathrm{La}_{2} \mathrm{O}_{3}$ and $\mathrm{Ni}-\mathrm{Ti}-\mathrm{Y}_{2} \mathrm{O}_{3}$ systems. The initial high-energy milling experiment conducted on $\mathrm{Ni}, \mathrm{Zr}$, and $\mathrm{La}_{2} \mathrm{O}_{3}$ powders successfully integrated $\mathrm{La}, \mathrm{Zr}$, and $\mathrm{O}$ in the Ni lattice of nanosize grains. Energy-filtered transmission electron microscopy revealed a high number density of nanosize particles in the Ni lattice and on grain boundaries following heat treatments at 800 and $750^{\circ} \mathrm{C}$. Chemical maps of $\mathrm{O}, \mathrm{Zr}$, and La confirmed that the nanosize particles, especially the larger particles, contained these elements.

\section{5: Molecular Fragment Databases for De Novo Structure-Based Design}

Benjamin P. Hay

\section{Project Description}

The objective of this project is to create a series of novel molecular-fragment libraries for use in computer-aided molecular design (CAMD) applications. These libraries, which will contain atomic coordinates and structural descriptors for tens of thousands of unique organic scaffolds, 
provide building blocks for constructing molecules on the computer using de novo structurebased design techniques. The process of generating the molecular-fragment libraries will be automated through the development of library-building software. This tool will operate by functionalizing an existing hydrocarbon library with synthetically attractive terminal groups such as ethers, amines, and amides. The utility of each new library will be verified through trial runs with the in-house CAMD tool HostDesigner. Methodology resulting from this study has broad potential applications. CAMD provides a rational approach to identify molecular architectures for radionuclide receptors used in nuclear separations (advanced nuclear energy systems), highly specific receptors for detection and analysis of chemical and biological agents (national security, medicine), model systems to study enzyme function (catalysis), and molecular components that self assemble to form functional materials (solid state lighting, solar energy, nanoscale materials).

\section{Mission Relevance}

The deliberate design of molecules and materials with desired physical and chemical properties is a current challenge that cuts across scientific disciplines. This project develops new CAMD capabilities to address this challenge. DOE Office of Science Basic Research Needs workshop reports, generated in support of recent initiatives, document pervasive opportunities for CAMD application. Examples include the design of actinide sequestering agents (advanced nuclear energy systems), molecular configurations for electric field enhancement (solid-state lighting), light absorbers, photovoltaics, and photoelectrodes (solar energy), and components for selfassembled nanostructured materials (nanoscience research). Results from this project have potential application in any research program that entails design of organic compounds and materials. Given that such activities occur in many research programs, broad potential exists for benefit to other federal agencies. Examples would be the design of molecular-scale machines for the National Science Foundation, magnetic resonance imaging agents for The National Institutes of Health, and components for nanoscale computer processors for the Defense Advanced Research Projects Agency.

\section{Results and Accomplishments}

Initial focus has been on the development of the computational tool required to automate the creation of new fragment libraries by adding functional groups to an existing hydrocarbon library. A beta version of this tool, a code named makelinks consisting of 14,564 lines of Fortran, has been compiled and is now being tested. Validation of performance with ether functional groups has been achieved by comparing makelinks output for specific test hydrocarbons against data generated systematically by hand and against output from conformational searches performed with commercial modeling software, PCModel. The first application of makelinks has been to create large libraries containing hydrocarbon fragments terminated with one or two ether oxygen atoms. With an input of 8,265 low-energy hydrocarbon fragments, makelinks has generated two new libraries containing 31,557 diether fragments and 30,335 mono-ether fragments. Test runs have demonstrated that the new ether libraries are compatible with the HostDesigner code. Application of makelinks to build libraries with amine-terminated fragments has been initiated. 


\section{0: Structure of Fluids Confined in Nanoporous Materials Using Neutron Scattering}

Gernot Rother and Ariel Chialvo

\section{Project Description}

The confinement of fluids in porous materials plays an important role in many natural and technical systems, for instance, carbon sequestration in rock formations and hydrogen storage for fuel cells. The properties of matter in confined spaces of nanoscopic size often deviate significantly from bulk because of sorption and finite size effects. Neutron scattering techniques are perfectly suited for the study of sorption effects, which allow for the first time the measurement of the mean density and volume fraction of the sorption phase. We propose to study the properties of the sorption phase systematically for different fluid-matrix combinations and different conditions of pressure and temperature. Experimental work will be complemented by molecular modeling studies to give detailed insight into the origins of the complex sorption patterns found in many systems with emphasis on high-density depletion effects.

\section{Mission Relevance}

Research on fluid-solid interactions under controlled conditions of pressure and temperature is central to a number of important nanoscience challenges receiving funding consideration from the DOE. Fundamental understanding of the structure and dynamics of fluids in confinement is currently lacking; therefore, successful completion of this project will promote future opportunities for follow-on funding in neutron-based nanoscience and produce new capabilities at ORNL for research. A Basic Energy Sciences (BES) call is anticipated in the area of Geosciences based on the recent BES-sponsored workshop "Basic Research Needs for Geosciences: Facilitating $21^{\text {st }}$ Century Energy Systems," wherein geological sequestration involving various scenarios of fluid-matrix interaction was identified as a key area requiring fundamental research.

\section{Results and Accomplishments}

During September FY 2007, the project's first month, progress was made in three areas. (1) a layout for the automated gas dosage system for neutron experiments in the pressure range of 0-1200 psi was developed; (2) the work on the manual high-pressure dosage system for simultaneous neutron scattering and volumetric sorption experiments in the pressure range of $0-10,000$ psi was completed. This setup will be used in a neutron reflectometry study of the sorption of $\mathrm{SF}_{6}$ to $\mathrm{Si} / \mathrm{SiO}_{2}$ at Hahn-Meitner Institute, Berlin in December 2007; (3) modeling work using molecular dynamics simulation techniques has been started on the system $\mathrm{H}_{2} \mathrm{O}$ in carbon slit pores.

\section{Publications}

Rother, G., et al., 2007. J. Phys. Chem. C 111, 15736. 


\title{
00223: Enhanced Cognizance of Evolving Threat Situations via Knowledge Discovery from Disparate Data
}

\author{
Auroop R. Ganguly, Vladimir Protopopescu, and George Ostrouchov
}

\section{Project Description}

The objective of this project was to develop new methodologies for knowledge discovery (KD) from disparate, dynamic data for adaptive predictive analysis and decision making in evolving threat situations. The premise of our approach was that evolving threat situations are often accompanied by signatures of abnormal, rare, and/or unusual events and that abrupt (unexpected) changes may be forewarned by hidden patterns in observables. These observables, in turn, could be inferred from continually refined models of "normal behavior" and statistical deviations from this behavior. We implemented this approach within a coordinated offline-online KD framework. The off-line part is a closed-loop, comprising disparate data integration, data analysis and unusual pattern detection, and process modeling. In this loop, disparate data are used to establish "normal" patterns and behaviors, which are then used in an on-line loop for detection of anomalies, abnormalities, or change in real-time, dynamic data.

\section{Mission Relevance}

Every federal agency is facing a huge stream of data that has to be distilled-sometimes very quickly - into consequential decisions. For instance, DOE deals with very complex problems ranging from climate change to the security of its physical and cyber infrastructure. DoD (e.g., the Defense Intelligence Agency, the U.S. Joint Forces Command, Army Research Laboratory), the Department of Homeland Security (DHS) (e.g., Science and Technology, Domestic Nuclear Detection Office), and the intelligence community are facing similar challenges. Large sets of data gathered from remote or in situ sensors, simulations, and network traffic need to be processed into actionable knowledge, and provided to decision makers for accurate, timely, and reliable decisions. This project addressed several of these important issues.

\section{Results and Accomplishments}

A suite of KD techniques have been developed, which include (1) automatic entity extraction from text-based graph stripping; (2) extensible database architecture for multimodal information integration; (3) new measures of "closeness" based on nonlinear manifold embedding and dimensionality reduction; (4) dynamic process modeling based on data acquired from cyberphysical sensor networks; (5) new goodness-of-fit metric and adaptive threshold measures for pattern classification and deviation detection; and (6) on-line change and anomaly detection from dynamic data. These techniques have been validated in the context of transportation corridor security and other applications. The primary insights obtained from our study are the following:

Transportation corridor insights include: improved discrimination between normal and abnormal truck profiles from static scale weigh station data; observation of a new power law behavior in truck speed distribution; evaluation of noise removal techniques from radiation measurements 
and extraction of the underlying signal without losing smaller, but possibly significant features; more refined analysis of radiation signatures from radiation sensors and better identification of shielded or camouflaged illicit radioactive material; and a proof of concept graphical user interface to showcase the above and display information from five disparate sensors for end-user vetting of anomalies.

Other application insights include: improved precision, recall, and computational efficiency of extracting named entities and their relationships from text data; faster and more user friendly queries on disparate knowledge bases; the possibility of behavior detection and discrimination from blog and e-mail data in simple dynamic social models; improved change and change-point analysis from time series and geospatial data with an application to remote sensing information; and more accurate anomaly and change-point detection and alarm generation based on adaptive thresholds in an online mode

\section{Publications}

Fang, Y. and A. R. Ganguly. 2007. "Mixtures of Probabilistic Principal Component Analyzers for Anomaly Detection.” ACM KDD 2007 - ACM Workshop on Knowledge Discovery from Sensor Data, August 12, 2007, San Jose, CA, USA.

Fang, Y. and A. R. Ganguly. 2008. Probabilistic Principal Component Analysis for Online Anomaly Detection with Application to Static Scale Data from the Watt Road Weigh Station. ORNL/TM-2006/546, Oak Ridge National Laboratory.

Ganguly, A. R., et al. 2007. "Knowledge Discovery from Sensor Data for Scientific Applications." In Learning from Data Streams-Processing Techniques in Sensor Networks, p.205. Springer Verlag, New York.

Ganguly, A. R., O. A. Omitaomu, and R. M. Walker. 2007. "Knowledge Discovery from Sensor Data for Security Applications." In Learning from Data Streams-Processing Techniques in Sensor Networks, p.187. Springer Verlag, New York.

Gerdes, D. A., S. Khan, and A. R. Ganguly. 2008. Nonlinear Dependence Measures with Application to Static Scale Data from the Watt Road Weigh Station. ORNL/TM2006/549, Oak Ridge National Laboratory.

Khan, S., et al. 2007. "Relative Performance of Mutual Information Estimation Methods for Quantifying the Dependence among Short and Noisy Data." Phys. Rev. E. 76, 026209-1.

Omitaomu, O. A. and A. R. Ganguly. 2008. Statistical and Wavelet-Based Denoising Methods for Analyzing Truck Radiation Data. ORNL/TM-2006/596, Oak Ridge National Laboratory.

Omitaomu, O. A. and A. R. Ganguly. 2008. A Methodology for Real-Time Decisions in Sampling of Trucks Based on Online Anomaly Analysis of Radiation Sensor Data. ORNL/TM2006/602, Oak Ridge National Laboratory.

Omitaomu, O. A. and A. R. Ganguly. 2008. Baseline Radiation Profiles and Alarm Generation Capabilities for Trucks Transporting Hazardous Cargo Using Radiation Sensor Observations and Model Simulations. ORNL/TM-2006/603, Oak Ridge National Laboratory.

Pan, C., P. Mitra, and A. R. Ganguly. 2007. "Spatio-Temporal Analysis on FEMA Situation Updates with Automated Information Extraction." ACM KDD 2007: ACM Workshop on Knowledge Discovery from Sensor Data, August 12, 2007, San Jose, CA, USA. 


\section{7: Detection and Identification of Bacteria and Viruses Including Stealth and Genetically Modified Organisms}

W. Hayes McDonald, Loren Hauser, Anthony V. Palumbo, Christopher W. Schadt, and Kevin J. Hart

\section{Project Description}

The ability to detect genetically modified threat agents is an emerging requirement for both defense and homeland security applications. Genetic engineering of bacteria to either enhance the pathogenicity of nonthreat agents or disguise the identity of threat agents should leave signatures. This project attempts to identify these signatures. These targets include virulence factors, toxin genes, insertion elements, and antibiotic resistance markers. In the first phase of this work, we have used surrogate organisms that can be handled at biosafety level (BSL) 1 or 2 (e.g., genetically engineered nonpathogenic bacteria) to develop protocols and strategies for detecting their genetic modification. These measurements are essential not only to determine sensitivity and specificity, but also to ascertain the knowledge base required to characterize the nature of these emerging threat agents. During the first two years of our effort we have demonstrated essential proof-of-principle experiments in detecting these emerging threat agents. As part of the process, we have begun to develop the algorithmic tools allowing us to select the most useful markers for detection and classification of potential threat agents. We have made significant strides in sample preparation, including a novel microwave-assisted acid hydrolysis protocol. As part of the process, we have begun to build databases of target genes and gene products using both publicly available and newly acquired information.

\section{Mission Relevance}

This work is relevant to solving a significant defense/homeland security challenge facing our country in the coming years-detecting genetically engineered biothreat agents. This project and its context within a broader system-of-systems approach to the next generation(s) of chemical and biological detectors lay the groundwork for rapidly detecting these and other threats.

\section{Results and Accomplishments}

During FY 2007, we made substantial progress on several of our originally outlined goals. In sample preparation, we developed a rapid, nonenzyme-based, microwave-assisted acid hydrolysis protocol and compared results from it to more standard trypsin-based protocols. Using these, we were able to detect genetically modified elements within an E. coli strain using separations as short as 20 minutes and to sample loads as low as the equivalent of $\sim 1,000,000$ cells. We have begun a parallel research effort in looking at DNA-based biomarkers by microarray. Initial work was a data mining exercise to develop a list of potential targets useful against a variety of elements likely to be present under conditions of genetic modification. Experimentally, we focused on a unified sample preparation protocol that will allow us to rapidly extract both protein/peptide and DNA-based biomarkers from a sample. The modeling has been undertaken in close coordination with our modeling efforts towards finding appropriate peptide biomarkers for identification and classification. Another algorithmic development for which we have spent a good deal of effort is looking at spectral matching. This is important because, even 
if appropriate peptides are chosen, it is the MS/MS spectrum of that peptide that is the unit of identification. Only by using robust and tuneable spectral matching will we be able to achieve sufficient sensitivity and selectivity to detect these signatures in complex, real-world backgrounds.

\title{
00226: Design and Synthesis of Novel Infrared-Active Nanophosphors
}

\author{
Linda Lewis, Lynn Boatner, Bob Smithwick, and Steve Allison
}

\section{Project Description}

Numerous federal agencies have identified a need for nanomaterials that emit infrared (IR) radiation with high fluorescent yields. Nanostructured materials are required for several reasons, including ink-jet printing tags for tagging, tracking, and locating; covertly marking and tracking materials of interest (e.g., nuclear components, combat ID, vehicles, illegal transactions); studying internal combustion in various engines to identify processes that increase fuel efficiency; enhancing luminescent light output due to increased surface-area-to-activator ratios for portable photovoltaic batteries; and supporting combat applications. Rare-earth-doped inorganic phosphors were targeted because of their inherent properties, including chemical, optical/UV, and radiation stability. Specifically, double-doped, rare-earth nanophosphors, known to increase luminescence output as a result of energy-transfer processes on a macro scale, were studied on a nanoscale and found in some cases to possess higher quantum efficiencies than their macro-sized counterparts. The luminescent properties of rare-earth-doped phosphors and glasses were evaluated with regard to doping levels, host matrix, and particle size. Systems activated by UV (solar), energetic electron, and optical interactions that result in the emission of IR at the desired wavelengths were successfully developed. The first-year objective was to design nanomaterials that support the needs identified above, while the second year objective focused on the nano-sized production of these materials and sensor-support applications.

\section{Mission Relevance}

The research conducted under this LDRD initiative is relevant to programs within the DOE IN-1, DOE Counterproliferation, DoD, and the Intelligence Community. The Office of the Secretary of Defense Counter Narcoterrorism Technology Program Office, and other agencies, are interested in materials based upon the near- and mid-IR glass phosphors. Numerous fact sheets describing materials developed under this research initiative have been disseminated throughout appropriate DoD agencies, and they have generated a tremendous amount of interest, resulting in significant follow-on funding. The materials developed have application to areas such as detection of improvised explosive devices, tagging, tracking, and locating (TTL) and long-life photovoltaic batteries. 


\section{Results and Accomplishments}

Differences between matrix compositions and dopant configurations (surface or embedded) in inorganic nano-phosphors significantly influence quantum-level properties, thus influencing electronic and optical characteristics. An array of solid-state materials were prepared through a variety of methods with various host matrices, dopant mixtures, sizes, surface/entrapped treatments, and distributions to study the effect of these variables on fluorescence-emission characteristics. Differences between matrix compositions and dopant configurations were screened on a nano-to-micron scale through combustions and sol-gel synthesis, a microscale through crystal growth, and macroscale through glass melting. Rare-earth elements active in the near- and mid-IR region were substituted into solid state, as well as amorphous host matrices, including rare-earth orthophosphates, YAG, alumina, complex aluminates, yttrium oxides, silicates, oxysulfides, garnets, and glass. A partial list of the materials prepared during this initiative include:

Sol Gel Synthesis: $\mathrm{CaAl}_{2} \mathrm{O}_{4}: \mathrm{Nd}(1 \%) ; \mathrm{CaAl}_{2} \mathrm{O}_{4}: \mathrm{Yb}(1 \%) ; \mathrm{Y}_{2} \mathrm{O}_{3}: \mathrm{Yb}(3 \%) ; \mathrm{Al}_{2} \mathrm{O}_{3}: \mathrm{Yb}(3 \%)$; $\mathrm{SiO}_{2}: \mathrm{Cr}(1 \%) ; \mathrm{SiO}_{2}: \mathrm{Mn}(1 \%)$; and an array of complex strontium aluminates triple doped with Er, Dy, and $\mathrm{Cr}$

Combustion Synthesis: $\mathrm{Al}_{2} \mathrm{O}_{3}: \mathrm{Yb}(3 \%) ; \mathrm{Al}_{2} \mathrm{O}_{3}: \mathrm{Yb}, \mathrm{Cr}(3 \%) ; \mathrm{Al}_{2} \mathrm{O}_{3}: \mathrm{Yb}, \mathrm{Mn}(0.25-3 \%)$; $\mathrm{Al}_{2} \mathrm{O}_{3}: \mathrm{Nd}(0.5-3 \%) ; \mathrm{Al}_{2} \mathrm{O}_{3}: \mathrm{Cr}(0.25-3 \%) ; \mathrm{Al}_{2} \mathrm{O}_{3}: \mathrm{Nd}, \mathrm{Cr}(0.5-5 \%)$; an array of doping combinations for $\mathrm{Al}_{2} \mathrm{O}_{3}$ : Ti, $\mathrm{Cr}$; $\mathrm{CaAl}_{2} \mathrm{O}_{3}: \mathrm{Nd}, \mathrm{Cr} ; \mathrm{Y}_{2} \mathrm{O}_{2} \mathrm{~S}: \mathrm{Yb}(3 \%) ; \mathrm{Er}_{0.08} \mathrm{Gd}_{1.92} \mathrm{O}_{2} \mathrm{~S}$ :, $\mathrm{Ti}(2 \%)$; $\mathrm{Al}_{2} \mathrm{O}_{3}: \mathrm{Eu}(1-2 \%)$; and array of $\mathrm{SrAl}_{2} \mathrm{O}_{4}: \mathrm{Eu}, \mathrm{Nd}$; etc.

Glass: $\mathrm{PbScPO}_{4}$ with $\mathrm{Er}(1,2,4,8,12 \%) ; \mathrm{PbInPO}_{4}$ with $\mathrm{Er}(1-8 \%) ; \mathrm{PbScPO}_{4}$ with an array of $\mathrm{Nd}$ and Ce doping levels

Crystals: $\mathrm{LuPO}_{4}: \mathrm{Er}(2,4,8 \%) ; \mathrm{YPO}_{4}: \mathrm{Er}(2,4,8 \%) ; 60 \mathrm{~g} \mathrm{PbHPO}_{4}+3.5 \mathrm{~g} \mathrm{Lu}_{2} \mathrm{O}_{3}+0.14 \mathrm{~g}$ $\mathrm{Er}_{2} \mathrm{O}_{3}(4 \%)+0.35 \mathrm{~g} \mathrm{Dy}_{2} \mathrm{O}_{3}(1 \%) ; 60 \mathrm{~g} \mathrm{PbHPO}_{4}+3.5 \mathrm{~g} \mathrm{Lu}_{2} \mathrm{O}_{3}+0.14 \mathrm{~g} \mathrm{Er}_{2} \mathrm{O}_{3}(4 \%)+0.035 \mathrm{~g}$ $\mathrm{Tm}_{2} \mathrm{O}_{3}(1 \%)$

Activation by solar radiation was one of the methods targeted for investigation. Several unique materials have been prepared via solid state, combustion, and sol-gel synthesis routes that hold promise for fulfilling the need for solar-activated tags. Activation efficiency by energetic electrons, cathodoluminescence, and radioluminescence have proven successful with highenergy electrons, neutron, X-rays, alpha and beta radiation. 


\section{7: Ensuring Dynamic Power Grid Stability: Integrated Electric and Information Grid Modeling}

Mallikarjun Shankar, James Nutaro, Phani Teja Kuruganti, Vladimir Protopopescu, and John Stovall

\section{Project Description}

The electric power systems industry is increasingly deploying modern information technology and algorithmic techniques to solve management and control problems in the distributed electric power grid. This project aims to show that computational techniques can help ensure the dynamic power grid's stability. The joint representation of the electric grid (analog and continuous) and the computational infrastructure (digital and discrete) requires information and control to cross the dividing line between the two infrastructures. We approach this problem by treating both infrastructures as network graphs in which the power network is mirrored by a distributed sensor network that carries sensor and actuation information (i.e., we consider the electric grid as a distributed network of physical devices, sensors, communication networks, and compute nodes). This approach allows us to use traditional computational techniques. By formulating electrical-grid-stability concerns such as frequency control during load-loss recovery as a distributed sense-response problem, we employ and evaluate algorithms that can prevent system degradation and collapse. We also support the computational approach by incorporating a user-directed discovery process based on data analysis and visualization. By compiling data sets from advertised outage databases, we are applying classification techniques to identify patterns in outage (and response) behavior. For the user-directed discovery process, we are developing geo-spatial visualizations of power system states and of the outage data sets.

\section{Mission Relevance}

New federal and industry initiatives for distributed power generation, distributed power storage, and demand-response-based market participation in power grid control make it increasingly urgent to examine the interaction between the power grid and its information backplane, and its consequent impacts on stability. Major power-generation companies and transmission and distribution operators are attempting to make the case for increased infrastructure investment and analysis directed at antiquated grid components following the Energy Policy Act (EPACT) of 2005. A set of arguments that justifies and highlights the importance of the infrastructure investment will emerge from this project. The research is relevant to the DOE Offices of Electricity Delivery and Energy Reliability (e.g., the Visualization and Controls thrust, GridWise-ModernGrid and their follow-on efforts) and Energy Efficiency and Renewable Energy. The results emerging from this project also have implications for critical infrastructure protection and assurance under the Department of Homeland Security. Recent research opportunity notices from state energy commissions (e.g., California Energy Commission) and from DOE suggest that our research is well aligned with strategic agency requirements.

\section{Results and Accomplishments}

We have significant results as shown in the following activity summary: (1) Modeling and Simulation: we developed scenarios for joint continuous and discrete event modeling and have 
created a tool for hybrid simulations called THYME (Toolkit for Hybrid-Systems Modeling and Evaluation) which has an associated ORNL/IDEAS \#05-403 Invention Disclosure filing.

(2) Data-Analysis: We reviewed the Disturbance Analysis Working Group data advertised by North-American Electric Reliability Council and created a scrubbed data set for analysis along two main axes. The first is to use well-known data-mining and visualization methods to identify anomalous or unexpected behavior. The second is to analyze the behavioral effects embedded in the outage data as extremes in the larger context of system faults. Our results suggest that lognormal plots may accurately characterize these outages, suggesting that we must revisit the widely held belief that power laws characterize the outages. (3) Algorithmic formulation: We have formulated the distributed response mechanism on the electric grid as a set of software modules (or agents). These agents will respond to sharp load variations (due to frequencyexcursion-caused generator shutdown or restart) by locally reacting to loads. (4) User-Oriented Discovery: We have developed software modules that enable situational awareness of the electric grid and enable targeted contingency analysis of regional failures (e.g., due to hurricanes).

\title{
Publications
}

Nutaro, J. J. 2005. “Constructing Multi-point Discrete Event Integration Schemes.” Winter Simulation Conference, December 4-7, 2005, Orlando, FL, USA.

Nutaro, J. J. 2007. "Discrete Event Simulation of Continuous Systems." In Handbook of Dynamic System Modeling, Chapter 11, pp.1-21. Chapman and Hall/CRC, Boca Raton.

Nutaro, J. J. 2007. "A Second-Order Accurate Adams-Bashforth Type Discrete Event Integration Scheme." Parallel and Distributed Simulation 2007, June 12, 2007, San Diego, CA, USA.

Nutaro, J. J., et al. 2007. "Integrated Hybrid-Simulation of Electric Power and Communications Systems.” 2007 IEEE Power Engineering Society General Meeting, June 24, 2007, Tampa, FL, USA.

Nutaro, J. J., P. Kuruganti, and M. Shankar. 2007. "Seamless Simulation of Hybrid Systems with Discrete Event Software Packages." 40th Annual Simulation Symposium (ANSS), March 25, 2007, Norfolk, VA, USA.

Nutaro, J. J., et al. 2006. "Integrated Modeling of the Electric Grid, Communications, and Control." ORMMES'06, September 6, 2006, Coimbra, Portugal.

Nutaro, J. J. and B. P. Zeigler. 2007. "On the Stability and Performance of Discrete Event Methods for Simulating Continuous Systems.” J. Comput. Phys. 227, 797.

\section{6: Combustion of Nanostructured Metal Fuels: Towards Designing Optimized Combustion Chambers}

\author{
M.P. Paranthaman, A.L. Qualls, B.G. Sumpter, and S.D. Labinov
}

\section{Project Description}

This project will create a new boron fuel for anaerobic combustion in unmanned underwater, high-altitude air vehicles, and possibly hypersonic engines, and conduct initial combustion proofing. This new high-power fuel will enable unprecedented capability in these small vehicles. 
The project is a refocused, narrower version of previous proposed projects and seeks development of one form of a new fuel for a particular high-performance application. An ongoing DoD aluminum combustor design is projected to have an in-vehicle energy density near $1200 \mathrm{Wh} /$ liter. In contrast, a boron-fueled stirling- or turbine-powered design can produce an energy density of greater than $2500 \mathrm{Wh} /$ liter because boron is more energetic and can be burned in a low-pressure power plant of lesser weight. The aluminum combustor uses $800^{\circ} \mathrm{F}$ steam in heavy, thick-walled pressure vessels, carries an oxidizer separately, and is projected to require a vehicle of 38 inches in diameter-much larger than most deployment platforms can handle. The more powerful but lighter boron plant will enable the use of smaller vehicles without sacrificing tactical capability and endurance, enable wider deployment options, and potentially be safer than aluminum-powered alternatives. Our scientific approach is based on previous ORNL work on the combustion of engineered clusters of metallic nanoparticles. Initial results have shown complete and rapid solid phase oxidation at temperatures of 1000-1400 K without chamber-fouling condensation or sintering_critical for multiuse, high-endurance engines.

\section{Mission Relevance}

DOE's overarching mission is to advance the national, economic, and energy security of the United States and minimize dependence on imported oil, which requires alternative energy sources and carriers. This project opens the possibility of an energy carrier and heat engines more powerful than both gasoline and internal combustion, and independent of the carbon cycle. While the project focuses on high-performance military applications of interest to DoD and the National Aeronautics and Space Administration, the follow-on implications for civil applications are enormous. While military designs may not be recyclable, civil ones could be done easily. Just as electricity moves energy from generation to point of use, nanostructured metal fuels can carry energy from centralized reduction facilities (using nuclear, coal, solar, geothermic, or water energy) to locations that need the transportation and portable power. As an alternative to fossil fuels, metal fuels would be safer, potentially easier to store and transport, have much greater power density, not pollute the environment during combustion, and be less costly to produce from metal ores than gasoline is from crude oil. This project addresses unfilled technology requirements for propulsion engines that have more power and energy density than those currently available so as to enable development of specific classes of unmanned vehicles. In particular, anaerobic engines could be developed that could be used in situations where either the induction of air or the exhaust of hot gas is foreclosed by either mission requirements or the operating environment.

\section{Results and Accomplishments}

During FY 2006-07, we have made significant progress in studying thermochemical properties and combustion behavior of metallic iron, aluminum and boron nanofuels. The self-ignition temperatures of these nanoparticles in pellet-shaped clusters were experimentally determined to be 520,820 , and $740 \mathrm{~K}$, respectively; and the possibility of oxygen adsorption on the nanoparticle surface to make an anaerobic fuel was theoretically predicted using the molecular dynamics calculations. Heat given off during the combustion of $1 \mathrm{~kg}$ of boron was determined to release about three times more energy per volume unit than the aluminum-water reaction under development by the Navy. New equations were derived to predict boron nanoparticle combustion dynamics, including size-temperature relationships. A working metal fuel combustor was also 
fabricated and successfully tested with aluminum/air and boron/air aerosols mixed with hydrocarbon fuel. Experimental results compared favorably with the mathematical model of a $1000 \mathrm{~W}$ metal fuel combustion chamber.

\section{Publications}

Sumpter, B. G., et al. 2007. "Solid-State Combustion of Metallic Nanoparticles: New Possibilities for an Alternative Energy Carrier.” J. Energ. Resour. Tech. 129, 29-32.

\section{4: NanoEPower_Nanocatalytic Direct-Fuel Thermoelectric Generator}

Zhiyu Hu, Thomas Thundat, and Chaitanya K. Narula

\section{Project Description}

The goal of this project is to develop a high-specific-power, long-operation-life, microelectromechanical systems (MEMS)-based solid-state direct-fuel electric power generation system which is mechanically simple, man-portable, and able to power electronics devices such as computers, sensors, and communication gear using fuels like methanol, ethanol, or hydrogen. Fuels for man-portable variants can be carried in small containers, much as cigarette lighter fluid was in the days of Zippo lighters, and potentially sustain soldiers in the field for days. The proposed system is based on ORNL's patent pending NanoEPower - a nanocatalytic thermoelectric generator. NanoEPower is a new class of solid-state power generation that operates at ambient temperature and pressure. During the reaction, the fuel's chemical energy spontaneously releases to thermal energy at predefined localized nanocatalytic heating zones without conventional ignition or high-temperature gas-phase burning/combustions while maintaining ambient bulk temperature. A nanocatalytic heating zone supported by a micromachined structure creates a microscale ultra-high thermal gradient. This gradient is two to three orders of magnitude greater than that in macroscale and enables high-efficiency direct conversion of nanoburning heat to electricity through a nanostructured thermoelectric heterostructure without moving parts.

\section{Mission Relevance}

This work closely relates to the overall DOE mission in improving energy utilization efficiency and clean energy efforts, which are supported by the White House and the U.S. Congress. At present, according to the International Energy Agency (IEA) 2007 report, over $90 \%$ of the world's energy comes from burning combustible fuels (such oil, coal, and natural gas) at high temperature $(>600 \mathrm{~K})$. The program offers an alternative path of direct energy conversion from fuels (such as methanol, ethanol, or hydrogen) to electrical power at nanoscale near room temperature on a nano-constructed solid-state device. The innovative and breakthrough approach allows us to build a new class of energy conversion system on a solid-state device without any moving parts. The success of this program could bring broad and deep impacts in the energy conversion concept-energy conversion at the nanoscale and low temperature. In practical 
application, our work has already attracted great interest from several DoD branches and DOE program managers.

\section{Results and Accomplishments}

In FY 2007, during the first year of this project, we initiated development of the three components that are essential to successfully demonstrate this technology: (1) nanocomposite thermoelectric thin films, (2) MEMS for the first-generation solid-state device structures, and (3) nanocatalytic heterostructures. To fabricate nanocomposite thermoelectric thin films, we used the e-beam co-evaporation method, which allows us to control the alloy mixture ratio, and the plasma sputter technique. We made nanocomposite p-type and n-type thermoelectric thin film structures using $\mathrm{Bi}, \mathrm{Te}, \mathrm{Sb}, \mathrm{Se}, \mathrm{Au}$, and other materials and measured several material properties, including the Seebeck coefficient, electrical resistivity, and carrier concentration. In MEMS platform development, we designed and fabricated structures for thermoelectric power generation. On a postage-stamp-size die (chip size: $16.5 \mathrm{~mm} \times 15 \mathrm{~mm} \times 0.5 \mathrm{~mm}$; weight: 0.25 $\mathrm{g}$ ), we integrated 4,500 microfabricated structures that are suspended in air a few micrometers above the silicon substrate. Two different methods were developed for patterning catalytic nanoparticles on various substrates: direct dispersion of prefabricated platinum nanoparticles and on-site synthesis via a chemical reaction. Nanocatalytic coating layers have been deposited on several different substrates such as silicon and glass. Auto-ignition of nanocatalytic reaction was achieved in methanol vapor/air mixture under ambient conditions.

\section{8: A Novel Process of Thick Nanocomposite Surfaces for Defense Applications}

Jun Qu, Zhili Feng, Peter J. Blau, Xun-Li Wang, Edgar Lara-Curzio, Hsin Wang, and Stan A. David

\section{Project Description}

Wear and friction are critical issues for many defense applications. The purpose of this work is to develop a novel surface engineering technique to form a highly wear-resistant nanocomposite surface for lightweight alloys, such as aluminum and titanium. Friction stir processing is used to stir and mix ceramic nano-sized particles into a metallic surface to form a nanocomposite layer to improve the surface hardness, strength, and wear resistance without sacrificing the bulk ductility and conductivity. Unlike most other surface engineering techniques, this process can form very thick layers, up to centimeters in thickness, avoiding delamination because of the inherent material continuity. This study is focused on developing the process and understanding the inherent mechanisms by taking advantage of the state-of-the-art characterization capabilities at ORNL to allow significant improvement in material properties. This surface engineering technique has great potential for many military applications to provide energy savings through friction and weight reductions. 


\title{
Mission Relevance
}

Friction and wear are estimated to cost $6 \%$ of the U.S, gross national product, or around $\$ 700$ billion annually. The terms "wear" and "friction" appeared 25 and 19 times, respectively, in the 2005 DoD Military Critical Technologies List (MCTL). This proposed nanocomposite surface engineering technique is expected to save energy through friction and weight reductions and to provide safer, longer-lasting, and more reliable service as a result of the harder, stronger, and more wear-resistant surfaces. The successful development of this technique will have great potential in aviation, ship machinery, and ground transportation applications. Particularly, this surface engineering process has great potential for many military applications that require highly wear-resistant surfaces, such as submarine mast fairing guiderails and mine countermeasures ship main propulsion shafts (NAVSEA), carrier landing cable-guiding sheaves (NAVAIR), lightweight armor (DARPA), etc.

\section{Results and Accomplishments}

The objective of this study is to develop a unique surface engineering process to form a hard, strong, and wear-resistant nanocomposite surface using friction stir processing (FSP). Unlike most other surface engineering techniques, FSP can form very thick layers, up to centimeters in thickness, and avoid delamination due to the inherent material continuity. In $\mathrm{FY} 07, \mathrm{Al}_{2} \mathrm{O}_{3}$ nanoparticles in two sizes ( 300 and $50 \mathrm{~nm}$ ) have been successfully stirred into a pure aluminum surface to form an $\mathrm{Al}-\mathrm{Al}_{2} \mathrm{O}_{3}$ nanocomposite layer with a particle concentration up to $20 \mathrm{vol} \%$. Compared with a nonprocessed aluminum surface, the nanocomposite surface demonstrated increased hardness (by $3 \times$ ) and yield strength (by $8 \times$ ), and reduced friction coefficient (by 55\%) and wear rate (by 200×). Residual stress mapping by neutron diffraction at the High Flux Isotope Reactor (HFIR) indicates mild tensile residual stresses of the aluminum matrix at the center of the processed zone in the longitudinal, traverse, and normal directions. Major challenges encountered in process development include the low densities of nanopowders and the high wear of conventional FSP tools. Powder placement methods and process parameters were tailored to improve particle dispersion and surface mechanical properties. New FSP tools were designed and fabricated to overcome the tool wear issue. Overall, substantial progress and very encouraging results have been achieved in the first year's research. However, challenges still remain such as nonhomogenous particle dispersion and thermo-mechanical cracking. This will require the second year's efforts on both process development and characterization to further improve material properties.

\section{5: Cognitive Radio for Transformational Logistics}

\author{
Mark A. Buckner, Michael R. Moore, Nageswara S. Rao, and Lawrence P. MacIntryre
}

\section{Project Description}

Our national transportation infrastructure, composed of complex, interdependent components, presents a particular challenge to national security because it is both a critical asset and a key vulnerability. To address this vulnerability, we propose cutting-edge information and 
communication technologies based on a synergy of novel algorithms and electronic circuits employed in cognitive radios (CRs). A CR can learn and dynamically adapt its communication parameters based on interaction with its environment through active negotiation with other spectrum users and/or passive sensing of the environment. Though not a requirement, most CRs are software based and implemented on software-defined radio platforms. For transportation security applications, CR technologies can provide the capabilities required of fourth-generation logistics tracking by adapting to nonexistent or rapidly changing infrastructures. Such systems will make use of a variety of standards based radio frequency identification and communications waveforms, global positioning system and other sensors, and computational intelligence to dynamically alter its waveform and routing schemes to facilitate connectivity over a variety of disparate communication links, including cell phones, satellite communications, and radio links. This capability will enable total in-transit visibility, automated generation of manifests (e-manifests), mission-based logistics responses, and automated repair scheduling.

\section{Mission Relevance}

This project is relevant to a variety of DOE national security missions including monitoring of special nuclear materials and critical infrastructure. Technology developed under this LDRD will provide military convoys with automated sensor and communication connectivity both among the trucks and back to oversight locations (e.g., command posts.) It will provide in-transit visibility for logistics' support missions. Domestically (e.g., DHS/Disaster Response), it will provide similar visibility and connectivity for convoys and teams that respond to large disasters in which the existing communication infrastructure has been destroyed and which may require, chemical, biological, radiological/nuclear, and explosive sensing. We anticipate the results of this project will also be of benefit to DOE's Spectrum Management program, NA-42, and several other federal agencies including CIA, DIA, FBI, NSA, DoD, DEA, ATF, DHS, DNDO, and NIJ, each with a variety of tagging, tracking and locating needs. Finally, the scientific/technical mission relevance involves the leap ahead in the amount of signal processing (communication at multiple layers plus sensor fusion) that can be achieved on small portable platforms.

\section{Results and Accomplishments}

We made significant progress in the following areas.

Waveforms. Three waveforms are now fully demonstrable on our software defined radio (SDR) platform: (1) Iridium paging, (2) Savi Radio Frequency ID (RFID), and (3) Wherenet RFID. The status of other key waveforms is as follows: (1) Iridium Short-Burst Data (SBD) - 90\% complete, (2) an ORNL custom waveform (DSSS-based) - 75\% complete, (3) Zigbee - $25 \%$ complete, and (4) 802.11b - 25\% complete. By the end of FY 2007, the best combination of these waveforms will be integrated to achieve our three-waveform milestone.

Sensor Integration. An appropriate chemical sensor system has been identified (developed under separate funding) for integration into this system. Actual integration will be conducted during year 2 as planned.

Cognitive Algorithm Development. This task had no year 1 goals. However, some related efforts in conjunction with the University of Tennessee have been initiated (see parameter optimization algorithm task below.) 
Mesh Network Routing Protocols. A trade-off study of available algorithms and resources was conducted. We investigated Zigbee, Bluetooth, and IEEE 802.11 as candidates for supporting physical mesh networking capabilities, and ORNL customized waveform methods will be investigated in year 2. Zigbee, IEEE 802.11, and ORNL customized waveforms have been chosen as possible candidates, but they are not unilaterally supported, and their capabilities are noncommensurate. This year we developed a framework that utilizes the native mesh networking capability of Zigbee combined with an Internet Protocol (IP)-based mesh protocol over IEEE 802.11 .

Parameter Optimization Algorithm. While this task had no year 1 goals, an initial effort was initiated with University of Tennessee staff (unfunded co-PIs for this LDRD) to explore an analog and digital circuit modeling and simulation framework that supports implementation of global parameter optimization algorithms. 



\title{
00201: In Situ, Time-Resolved Neutron Diffraction Study of Materials Behavior under Severe Thermomechanical Deformation
}

\author{
Zhili Feng, Wan C. Woo, Xun-Li Wang, Camden R. Hubbard, Stan A. David, B. Radhakrishnan,
} and Gorti Sarma

\section{Project Description}

The microstructure evolution of materials under rapid and severe thermomechanical deformation is one of the most important yet least understood areas in materials science and engineering. A major contributing factor has been the lack of direct in situ observation and determination of the temperature, stress, and microstructure changes as they evolve rapidly under complex thermomechanical material synthesis environment. This project is aimed at addressing this challenge by developing a novel neutron-scattering measurement and data analysis approach that would enable unique in situ, time-resolved measurement of material behavior under severe thermomechanical loading conditions, a completely new area of application for neutron scattering in materials science and engineering. The program builds upon the following key technical tasks: (1) a novel in situ data collection technique that would drastically improve the temporal resolution of neutron scattering, thereby allowing for time-resolved material studies; (2) a portable thermomechanical processing system that can be used as a platform for controlled severe thermomechanical conditioning of materials; (3) new data-processing and interpretation techniques to decode the temperature, stress, and microstructure information embedded in the neutron scattering data; and (4) in situ measurement of stress, temperature, and microstructure changes in aluminum and magnesium alloys.

We have successfully demonstrated the novel neutron scattering approach for in situ, timeresolved neutron measurement for the temperature, stress, dislocation density and crystallite size of metals as function of process conditions. The new measurement methodology has attracted considerable attention in the materials science and neutron-scattering community.

\section{Mission Relevance}

This project is relevant to the DOE Office of Basic Energy Sciences Neutron Science and Materials Science and Engineering programs, as it leads to a novel use of neutron scattering to understand the fundamentals of materials behavior. Our technique will provide a better understanding of the intricate interplay of stresses, temperature, and microstructures in complex metal processing and synthesis, which would be important to a wide range of advanced structural and functional materials for DOE Energy Efficiency, Fusion Energy, and Fossil Energy programs. Successful demonstration of the in situ neutron-scattering capability would also enable us to enhance materials research and development being conducted by other federal agencies and U.S. industry. We expect (1) a new and active academic and industrial user base for in situ, timeresolved neutron scattering studies of material behavior, (2) basic materials science research programs to develop the theoretical basis for novel approaches to produce unique microstructures 
in structural and functional materials, and (3) applied research programs from U.S. industries where thermomechanical processing of materials is critical to their business.

\section{Results and Accomplishments}

Significant progress has been made. In FY 2005 and FY 2006, we confirmed the existence of the quasi-steady-state condition in our experiments. We demonstrated that, under the quasi-steadystate condition, neutron collection time is independent of the rate of change of material behavior during the transient. This makes it possible to select the neutron collection time based purely on the neutron flux and sampling volume requirement of a test, thereby circumventing the neutron flux limitations for studying fast transient material behaviors. We developed a neutron diffraction data analysis methodology to allow for simultaneous decoding of the transient temperature and stress from the neutron measurement data for a two-dimensional case, without the need for additional auxiliary measurement.

The FY 2007 activity focused on (1) decoding the microstructural changes during severe thermomechanical deformation and (2) relating these changes to the temperature and stress conditions under which these changes take place. We obtained a quantitative measurement of the drastic changes in dislocation density and crystallite size during thermomechanical deformation imposed by the friction stir process. The formation of nanocrystallite and its dynamic growth were also observed for the first time.

The insitu neutron scattering approach developed in this LDRD has a unique feature - the temporal resolution actually increases as the neutron-scattering volume decreases. Therefore, it is particularly suitable for understanding the fast-changing and highly nonuniform material behavior in real-world material processing and synthesizing conditions where conventional neutron-scattering techniques are not effective.

\section{Publications}

Feng, Z., et al. 2007. "In-situ Neutron Diffraction Measurements of Temperature and Stresses during Friction Stir Welding of 6061-T6 Aluminum Alloy.” Sci. Technol. Weld. Joining 12, 298.

Woo, W., et al. 2007. "Quasi-Steady State Principle and In-Situ Real-Time Investigation of Transient Strains in 6061-T6 Al Alloy Using Neutron Diffraction." 10th International Conference on the Mechanical Behavior of Materials. 345-346, 797-800.

Woo, W., et al. 2006. "Feasibility of Thermal Strain Measurements during Quasi-Steady State Using Neutron Diffraction.” ECRS7, September 13-17, 2006, Berlin, Germany.

Woo, W., et al. 2006. "In-Situ Neutron Difraction Measurement of Transient Temperature and Stress Fields in a Thin Plate." ECRS7 Conference, September 13, 2006, Berlin, Germany.

Woo, W., et al. 2006. "In-Situ Neutorn Difraction Measurement of Transient Temperature and Stress Fields in a Thin Plate." Appl. Phys. Lett. 88, 1-3.

Woo, W., et al. 2007. "In-Situ Neutron Diffraction Measurements of Temperature and Stresses during Friction Stir Welding of 6061-T6 Aluminum Alloy." Sci. Technol. Weld. Joining 12, 298. 


\section{2: Time-Resolved Analyses of Microstructure in Advanced Materials under High Magnetic Fields Using Neutrons}

Gerard M. Ludtka, John B. Wilgen, Roger A. Kisner, Gail Mackiewicz-Ludtka, Jaime A. Fernandez-Baca, and Camden R. Hubbard

\section{Project Description}

Fundamental science breakthroughs are being facilitated by high magnetic field studies in a broad spectrum of research disciplines. Furthermore, processing of materials under high magnetic fields is a novel technique with very high science and technological potential. However, there did not exist the capability to do in situ, time-resolved neutron scattering experiments at high magnetic field strengths and at elevated temperatures. Therefore, prior measurements were performed ex situ and did not capture the microstructural evolution of the samples during high field exposure. To address this deficiency, we established an elevatedtemperature, high-magnetic-field processing and analyses system at the High Flux Isotope Reactor (HFIR) which links the analytical capabilities inherent in neutron science to the needs of magnetic processing research. To achieve the research initiative goals, we designed, fabricated, and demonstrated a high magnetic field instrument insert that provides the thermal processing and auxiliary instrumentation environment needed to run ultrahigh magnetic field processing experiments at the HFIR on the wide angle neutron diffractometer (WAND) and Neutron Residual Stress Facility 2 (NRSF2) instruments. Our goal is to be the first team to apply advanced neutron scattering to explore time-resolved characterizations of magnetically driven alloy phase transformations and determination of ordering and magnetic moments in an alloy under transient conditions. This enhanced, in situ time-resolved materials characterization capability will be an enabler of basic science research opportunities. This work also established a research instrument and environment for international community users at the HFIR under the ORNL neutron science program.

\section{Mission Relevance}

Our successful accomplishment of this research is providing a unique, world-leading neutron scattering instrument environmental system at the HFIR facilitating high-temperature, high magnetic field research that will result in major scientific breakthroughs, in understanding fundamental material behavior, in advancing magnetic field-based, energy-saving processing methods, and in developing the materials systems of the future across a broad range of scientific disciplines. This endeavor established the environmental system at the HFIR on the WAND and NRSF2 instruments and therefore will become a critical component of the HFIR user program for collaborative neutron science research. Therefore, very basic applied research programs utilizing this unique capability are anticipated to be funded through the DOE Office of Science energy programs, Defense Advanced Research Projects Agency, National Institutes of Health, National Aeronautics and Space Administration, National Science Foundation, and industry through the many research opportunities that high magnetic field processing at elevated temperature coupled with neutron science will spawn. 


\title{
Results and Accomplishments
}

The significant accomplishments of this project include (1) establishing unique world-leading facilities/capabilities at the HFIR, enabling matter to be probed simultaneously at elevated temperature and under high magnetic fields using neutrons and (2) achieving unprecedented insitu analyses in advanced materials under high magnetic fields while at temperature. This involved designing, fabricating, and demonstrating a sample environment capable of (1) programmable thermal transient or steady-state temperatures up to $1500 \mathrm{~K}$, (2) interfacing this thermal-magnetic system with the existing 5-T superconducting (SC) magnet system and inserting them into the WAND and NRSF2 instruments at the HFIR, and (3) conducting experiments on ultrahigh purity Fe-C binary alloys that will lead to the generation and publication of the first three-dimensional (temperature-composition-magnetic field strength) phase diagrams for these materials.

The successful completion of this project of uniquely integrating neutron probing capability with a thermal-magnetic environment is having a major impact in stimulating new areas of research and attracting new users from both the academic and industrial communities. This facility is enabling a totally new materials processing paradigm to be investigated to develop the next generation of structural and functional materials. In addition, these capabilities to do precisely controlled science investigations at elevated temperature and magnetic field complements the MS\&T Division's 9-T SC Magnet Thermal-Magnetic Processing facility by enabling the fundamental mechanisms behind material performance improvements to be investigated, understood, and optimized for "Science to Energy" breakthroughs.

\section{Publications}

Ludtka, G. M., et al. 2007. “Time-Resolved Analyses of Microstructure in Advanced Materials under Magnetic Fields at Elevated Temperatures Using Neutrons." 2007 TMS Annual Meeting \& Exhibition, February 25 - March 1, 2007, Orlando, FL, USA.

\section{1: Infrastructure Development for Neutron Scattering for Biomembranes and Biomimetic Membranes}

\author{
William T. Heller, Yiming Mo, Dean A. A. Myles, Greg S. Smith, and John F. Ankner
}

\section{Project Description}

Biological membranes and biomimetic systems are topologically complex and incredibly dynamic systems where function is largely effected through complex interactions between components of the system, such as lipids and proteins in biological membranes. The study of biological membranes and biomimetic systems is important to a variety of scientific disciplines, and it is an area of key strategic interest to DOE and National Institutes of Health (NIH). ORNL currently lacks the infrastructure to effectively support this science at its two neutron sources. We propose to develop the preparative capabilities, sample environments, analytic and modeling tools, and the core expertise required to support a strategic program of biomembrane, membrane 
protein, and biomimetic membrane research at ORNL and facilitate user community science at the Spallation Neutron Source (SNS) and High Flux Isotope Reactor (HFIR). Specifically, we will establish facilities and expertise for the expression and purification of membrane proteins and sample preparation for neutron reflectometry experiments. Additionally, sample environments for the neutron reflectometers will be developed that are optimized for investigations of the structure and function of biological membranes and biomimetic systems. Data analysis and modeling tools needed to interpret the data collected from these complex systems will be identified. We will demonstrate these capabilities by studying the association of specific lipids with the membrane-integral protein porin. Establishing this platform and capability at ORNL will leave us optimally positioned to contribute to the programmatic goals of DOE and NIH in this exciting field of science.

\section{Mission Relevance}

The project addresses mission goals of the DOE Office of Biological and Environmental Research's Genomics: Genomes to Life by developing the sample preparation and neutronscattering infrastructure needed to develop a molecular-level understanding of biomembranes, a critical and complex system possessed by every living cell, and biomimetic systems having technological applications. The work is also relevant to the missions of the NIH. The NIH's Structural Biology Roadmap resulted in the programs Membrane Protein Production and Structure Determination (RM-04-026) and Centers for Innovation in Membrane Protein Production (RFA-RM-04-009). The NIH program call (PA-06-119) for studies of the structural biology of membrane proteins is a continuation of a long-running program announcement serving several institutes of the NIH. By providing the infrastructure that researchers need, we will make it possible to use neutron scattering as another structural characterization tool for studying these challenging systems.

\section{Results and Accomplishments}

Good progress was made during FY 2007. A sample environment designed during FY 2006 was produced with the assistance of the Sample Environment Group of the SNS. A second design of a sample cell for the liquids reflectometer that incorporates active temperature and hydration control was developed and fabricated. Assembling the infrastructure needed for membrane protein expression and purification, as well as for neutron reflectometry sample preparation, was driven by Dr. Yiming Mo, who joined the project in April 2006. The group continues to interact with the Distributed Data Analysis for Neutron Scattering Experiments project to identify tools for the analysis and modeling of neutron reflectometry data. The demonstration produced hydrogenated and deuterated porin, a membrane protein to be used for neutron-scattering studies. Experiments to characterize the membrane proteins before reconstitution into model membranes for neutron reflectometry studies produced interesting results that have broader implications for studies of detergent-associated membrane proteins. A manuscript describing the results is in preparation. Neutron reflectometry experiments were performed at the National Institute of Standards and Technology using the Liquids Reflectometer of the SNS. The results, which suggest a phase separation in charged/neutral lipid mixtures, point the way forward for continued work on the demonstration project during FY 2008. 


\title{
00004: Small-Angle Neutron Scattering Investigations and Computational Modeling of Creep Cavitation in Nanoparticle- Strengthened Materials
}

\author{
G. Muralidharan, M. Agamalian, R.L. Klueh, Weiju Ren, J. P. Shingledecker, M. L. Santella, \\ B. Radhakrishnan, and G. B. Sarma
}

\section{Project Description}

Ferritic/martensitic steels such as P22, P91, P92, and P122 are typically strengthened by nanosized precipitate particles that include carbides and carbo-nitrides. Creep void formation (cavitation) occurs at prior austenite grain boundaries and precipitate interfaces during highstress, high-temperature service; this phenomenon eventually leads to cracking in the finegrained heat-affected zone (HAZ) of weldments of these steels and in the base metal, which can severely limit their service lifetimes. Hence, there is a critical need to understand the role of various microstructural features that clearly affect cavitation. Small-angle neutron scattering (SANS) and ultra-small-angle scattering (USANS) combined with electron microscopy and computational modeling, has the potential to develop the much-needed understanding of the process of cavity nucleation and growth. Computational modeling that incorporates the effects of grain and precipitate structure, as well as the spatial distribution of cavity nucleation (from the loading history), will enable interpretation of results obtained from the SANS measurements. The purpose of this project is to develop SANS and USANS techniques and supporting computational modeling approaches to help understand creep cavitation and to establish a broadly integrated capability to conduct research on a number of other important problems requiring intimate knowledge of microstructural processes.

\section{Mission Relevance}

Ferritic-martensitic steels are candidate materials for use in various energy systems, including Generation IV nuclear reactors, ultra-supercritical (USC) steam boilers, fusion reactor first-wall and blanket structures, heat-recovery steam generators, turbines, and fuel cells. This research project emphasizes the application of SANS and computational modeling in solving problems relevant to these next-generation energy sources and is consistent with the DOE missions in energy resources and science. DOE's nuclear reactor, industrial technologies, fossil energy, and hydrogen programs and the Office of Science are expected to benefit from this research. Success in the synergistic approach proposed to address fundamental aspects of materials performance controlled by phenomena that span multiple length scales will enable the Laboratory to actively pursue many areas of materials research and development related to processes that involve high temperatures and/or fine-scale structures. Materials-related programs that are part of other federal agencies, such as the DoD, also are likely to benefit from the results from this work

\section{Results and Accomplishments}

Significant emphasis was placed on correlating microstructures observed through optical and electron microscopy techniques to results from USANS/SANS/USAXS measurements in an experimental grade of Fe-9Cr ferritic-martensitic steel subjected to several heat-treatments but had not developed creep cavities. It was observed that results from the analysis of the scattering 
data could be successfully correlated with the intrinsic characteristics of the martensitic structure observed through transmission electron microscopy. It was also verified that the scattering observed from nanosize precipitates resulting from heat-treatment (tempering) of martensite was separable from that of the martensitic matrix.

A three-dimensional finite element model based on crystal plasticity was used to examine the influence of precipitates located at grain boundaries and triple junctions on the accumulated plastic strain and hydrostatic stress distributions after cyclic loading at high temperatures, because nucleation and growth of creep cavities are often related to a critical plastic strain and gradients in hydrostatic stress. The simulation results indicate the existence of higher strains at certain grain boundaries, and even higher values close to the precipitates. Also, the introduction of a precipitate causes a shift in the location of the highest stress values to regions within the precipitate due to its high modulus. The mean stress values in the matrix adjacent to the precipitate are lower for a spherical precipitate compared to the cube-shaped precipitate, while the plastic strain values are higher.

Creep-fatigue tests were successfully carried out on $2.25 \mathrm{Cr}-1 \mathrm{Mo}$ steel specimens to study the development of creep cavities. Study of time-dependent accumulation of damage was facilitated by performing fatigue tests on separate specimens for two different lengths of time. USANS/USAXS data were obtained from samples prepared from the fatigued specimens. Analysis of data using techniques developed in the project shows that cavity nucleation and growth had not occurred during the test times adopted for the fatigue experiments.

\section{5: Use of Small Angle Neutron Scattering (SANS) to Study Complex Systems}

Kenneth C. Littrell

\section{Project Description}

Many different systems exhibit changes to structure and order on length scales ranging from nanometers to microns. Examples of such systems include complex fluids, catalytic materials, nanocomposites, coals and oil-bearing shales, metallic alloys and novel magnetic materials. These systems are important in medicine and food science, energy and environmental remediation, and industry and engineering. Small-angle neutron scattering (SANS) provides information that is complementary to and not readily measured by other techniques. We propose to develop the techniques, ancillary equipment, and software necessary to utilize the SANS1 Small Angle Neutron Scattering instrument at the High Flux Isotope Reactor (HFIR) to probe the formation, morphology, and interactions of the nanoscale structures present in complex systems in tunable environments. In particular, we will study the phase diagram of actinide solventextraction systems related to the PUREX (Plutonium and URanium Extraction) process by SANS, develop data analysis software for this and related systems, develop high-speed, highaccuracy approximations to numerically integrated SANS models of cylinders and similar structures derived from them, and develop and test expressions for the resolution function for a 
lens SANS instrument, both single-axis and anisotropic 2-D, including the effects of gravity, lens thickness, and lens-sample separation, and design ancillary equipment for the SANS instrument to accommodate these experiments.

\section{Mission Relevance}

The solvent extraction work directly addresses DOE missions in energy resources, nuclear security, and environmental quality by helping close the circle in the nuclear fuel cycle. The other aspects of the project support this work and DOE missions in providing top-quality scientific service to the user community in neutron science. The developments that result from this program will substantially enhance the benefits of the availability of SANS to the Scaling of Structure and Properties Research Focus Area in the Center for Nanoscale Materials Science (CNMS) for probing structures on nanometer to micron length scales on site and for experiments that would not be possible or practical at other domestic facilities due to flux limitations. The form factor calculations will also benefit DOE synchrotron (and lab instrument)-based small angle X-ray scattering (SAXS) user program and science initiatives.

\section{Results and Accomplishments}

During FY 2007, the project's first year, we made significant progress in several of the areas covered by this proposal. (1) The results of measurements of the 1-D polarizing lens were compared with new calculations of the anticipated lens performance and published; these results also were a significant part of a contributed presentation at CANSAS 14 in Dongguan China in April 2007. (2) The cylindrical form factor results have been tested over a wide range of input parameters and distributed to the SANS instrument science community. These results were presented in a contributed presentation in June 2007 at the European Conference on Neutron Scattering in Lund Sweden. (3) We have calculated the lens resolution function for anisotropic, Cartesian data. Results were presented in invited tutorials at the First U.S.-China Neutron Scattering Collaboration meeting in Beijing in November 2006 and in an invited colloquium at CIAE in China in April 2007 and in contributed presentations in March in Villingen, Switzerland at the NOP 07 international workshop and at CANSAS-V in Gaithersburg in October 2007. (4) The open-literature published results on the PUREX system were presented in an invited colloquium at CIAE November 2006. (5) The IGOR-based data reduction and analysis package was brought into agreement with best-practices standards of the IGOR SANS programming community and disseminated to the users. It is now the installed on the instrument computers for user-operation experiments and is being adapted for remote access through XCAMS. This package was also presented at CANSAS-V in Gaithersburg, MD, in October 2007.

\section{Publications}

Littrell, K. C., S. G. T.Velthuis, G. P. Felcher, et al. 2007. "Magnetic Compound Refractive Lens for Focusing and Polarizing Cold Neutron Beams." Review of Scientific Instruments 78, March. 


\section{3: High-Temperature, High-Pressure Studies of Dynamics of Fluids in Nanopores using the SNS Backscattering Spectrometer}

Eugene Mamontov, David R. Cole, Peter T. Cummings, Kenneth W. Herwig, and Louis J. Santodonato

\section{Project Description}

The purpose of this project is to determine the key features of dynamics of water and aqueous solutions confined within nanoporous materials under the extreme temperature-pressure conditions encountered outside the explored regions of the phase diagram. We will achieve this goal by means of designing unique sample environment equipment and utilizing the best-in-class neutron spectrometer (BASIS, SNS), which have unprecedented capabilities. We will perform scattering experiments and computer simulations to quantitatively assess molecular properties of confined fluids. Successful development of this project will demonstrate the capabilities of the first operational Spallation Neutron Source (SNS) neutron spectrometer to a broad group of prospective instrument users and showcase the capabilities of the SNS to conduct research at the forefront of chemical physics, physical chemistry, and materials science.

\section{Mission Relevance}

This project is directly relevant to DOE's energy resources mission. The need for characterization of potential hydrogen-storage materials, including nanoporous oxides at extreme conditions, was described in the document derived from the BES-sponsored workshop on Basic Research Needs for the Hydrogen Economy. A preliminary draft agenda for the next BESsponsored workshop on Basic Research Needs for Advanced Nuclear Energy Systems indicates that investigations of materials and chemical systems under extreme conditions are two of the six thematic science areas to be addressed. In addition, users of the SNS will use the capabilities developed in the course of the project. Thus, research activities supported by the federal agencies other than DOE (e.g., National Science Foundation) will benefit from this project.

\section{Results and Accomplishments}

During FY 2007, the project's first year, we made significant progress in several areas. We carried out quasielastic neutron scattering (QENS) experiments on the high-flux backscattering spectrometer at the National Institute of Standards and Technology Center for Neutron Research. Two types of silica were used as confining matrices: MCM-41 with a pore size of $27 \AA$ and heattreated SBA-15 with a pore size of approximately $8 \AA$. The latter material was obtained by heat treating standard SBA-15 that resulted in collapsing of the larger pores, leaving only the $8 \AA$ pores. We believe that heat-treated SBA-15 may possess excellent hydrothermal stability, thus making it an excellent candidate for high-temperature, high-pressure studies. Our results have demonstrated that the diffusion dynamics is suppressed to a much higher extent in the $8 \AA$ pores of SBA-15 compared to the $27 \AA$ pores of MCM-41. This makes the heat-treated SBA-15 the system of choice for the future QENS experiments on the BASIS at the SNS. While it is possible for the diffusion dynamics of aqueous solutions confined in MCM-41 to become too fast for the BASIS at higher temperatures (even with a much wide dynamic range of the BASIS compared to the high-flux backscattering spectronometer, the dynamics of those confined in SBA-15 should 
remain in the dynamic range of the spectrometer. A second area of progress is the design of parts of the high-temperature, high-pressure system that have been finalized and ordered from the vendors. These parts include sapphire bodies of the pressure cells, cell flanges, gas-handling system, pressure intensifier, and the dedicated furnace. Another area of progress is the start of MD simulations of water in the pores of SBA-15 at ambient conditions; we are proceeding with MD simulation of water and aqueous solutions at high temperatures and high pressures using our highly parallel simulation software.

\section{5: A Robust Polymer Scaffold System for Bioinspired Membranes}

John F. Ankner, S. Michael Kilbey, II, Jimmy Mays, Jamie M. Messman, and Dean A. Myles

\section{Project Description}

The objective of this project is to develop expertise in the preparation and investigation of using neutron scattering of soft material scaffolds that can be used to create bioinspired membranes. Interfaces play a key role in biological processes, and many biological molecules function at interfaces and in confined environments. To address these broad aims, the polymeric system we are investigating is an amphiphilic block copolymer comprising a short hydrophobic polystyrene (PS) block and a longer hydrophilic and reactive block that consists of a random copolymer containing poly(vinyldimethylazlactone) (PVDMA). This project comprises four general tasks: (1) synthesis of VDMA-containing amphiphilic block copolymers, (2) assembly of these copolymers into layers, (3) chemical modification of deposited layers and peptide attachment, and (4) neutron reflectivity measurements of the films at all stages of preparation.

\section{Mission Relevance}

Developing robust platforms that can be used to examine structure-property-function relationships of biomolecules attached to and penetrating into interfaces is crucial to ORNL's taking a leadership role in bridging biology and biological processes to the development of nextgeneration materials, devices, and processes.

\section{Results and Accomplishments}

During FY 2007, the project's first year, we made significant progress in polymer synthesis and deposition: (1) We utilized a controlled free radical polymerization technique known as reversible addition-fragmentation chain transfer (RAFT) to mediate the synthesis of 2-vinyl-4,4dimethylazlactone (VDMA) containing (co)polymers, (2) we successfully functionalized VDMA-containing copolymers using simple chemicals (e.g., $n$-hexyl amine) for proof of concept. Additionally, we were able to monitor the functionalization of VDMA-containing copolymers using real-time in situ remote probe ATR-FTIR spectroscopy, (3) we used extensive polymer characterization techniques to evaluate structure-property-function relationships. For example, we employed size exclusion chromatography (SEC) to evaluate the molecular weight and distribution of molecular weights. Nuclear magnetic resonance spectroscopy (NMR) 
provided valuable information regarding copolymer structure. Dynamic and static light scattering was used to determine particle size and shape. Thermal assays, such as differential scanning calorimetry (DSC) and thermogravimetric analysis (TGA), have yielded information pertaining to (co)polymer thermal transitions and decomposition, respectively. The SNS liquids reflectometer has been commissioned in FY 2007. We have performed preliminary characterization of polymer films and have developed solid/liquid cells to observe in situ the structural changes induced at the polymer-solution interface. Finally, we identified several promising candidate proteins for deposition tests. We will focus efforts in the second year of the project on expressing one or more of these proteins and in attaching appropriate receptor peptides to the VDMA films.

\section{Publications}

Messman, J. M., A. Banaszek, J. Barringer, J. W. Mays, S. M. Kilbey, II, "Synthesis, Assembly, and Biofunctionalization of Stimuli-Responsive Polymer Brushes," in Proceedings of the $34^{\text {th }}$ Annual International Waterborne, High-Solids and Powder Coatings Symposium, 77-88, 2007.

\section{9: Probing Molecular Interaction between Microbial-Cell Protein and Mineral Surfaces with Neutrons}

Liyan Liang, Baohua Gu, J. Anker, D. Myles, W. Wang, and A. Johs

\section{Project Description}

The purpose of the project is to investigate interactions between microbial-cell proteins and mineral surfaces, which are critical to understanding the electron transfer mechanisms and microbial ecosystem reactions/responses in subsurface environments. We hypothesize that direct contact between microbial-cell proteins and minerals is necessary for electron transfer along a respiratory chain to mineral iron. We use neutron reflectometry in combination with aqueous chemical and surface analytical techniques to study the effects of surface charge, chemical affinity, and specific hydrophobic/hydrophilic interactions on binding between mineral functional groups and cell proteins when embedded in membrane lipids. Iron oxide nanoparticle films and cytochrome proteins derived from the outer walls of microbial cells (e.g., sulfate or iron-reducing bacteria) are used as a model for the study.

\section{Mission Relevance}

This project is relevant to DOE's Genomics: GTL and Environmental Remediation Sciences Program (ERSP) programs. Contributions to understanding the process of electron transfer and mineral respiration is related directly to Genomics: GTL program. Microbial and mineral interaction has profound implication to bioremediation and therefore is relevant to the DOE ERSP program. This project should be beneficial to several National Science Foundation (NSF) and National Institutes of Health $(\mathrm{NIH})$ programs: including NSF programs on Cellular Systems and the Interagency Opportunities in Multi-Scale Modeling in Biomedical, Biological, and 
Behavioral Systems, and NIH programs on Research on Microbial Biofilms and Quantitative Approaches to the Analysis of Complex Biological Systems.

\section{Results and Accomplishments}

The first year effort focused on the following: (1) isolate and purify sufficient quantities of cytochrome proteins from pure cultures of Geobacter sulfurreducens and acidiphilic Fe-reducing bacteria, Acidiphilium cryptum; (2) develop techniques for forming iron oxide film on $\mathrm{Si}$ substrate and perform neutron reflectivity (NR) measurements to set a baseline for subsequent study; and (3) prepare lipid films on Si substrates and create suitable interfacial and computational models for interpreting NR data and for future work. We have accomplished all these initial tasks. We isolated and purified a type of soluble protein, PPCA, with continuing efforts in obtaining a membrane protein. We were successful in producing iron oxide thin films with $\sim 60 \%$ coverage. NR measurements were made for the iron oxide thin films, as well as for the membrane lipids layers. In addition, a cyclic voltammetry technique is being developed for measuring the redox reactions between iron oxide and cytochrome proteins for small sample quantities.

\section{2: High Throughput Neutron Crystallography for Macromolecular Structure, Function and Design}

Dean Myles, Hugh O’Neill, and Edward Snell

\section{Project Description}

This project addresses the last remaining challenge and bottleneck in neutron protein crystallography - the rational growth of single crystals of soluble and membrane bound proteins that are suitable for neutron analysis. Traditional screening methods are inefficient, slow, and labor intensive, often with long lag times of 2-4 years before successful structure determination. We aim to radically transform this process by harnessing the high throughput, robotic protein crystallization screening techniques that have transformed crystal growth in the structural genomics world. The effects of varying multiple parameters can be screened and evaluated rapidly, systematically and with dozens of proteins assayed against 1000s of variables at the same time. Working with colleagues at the Hauptman-Woodward Medical Research Institute, we will tackle at least 30 different protein systems, including cellular, signaling, and membranebound proteins and enzymes of interest in medical, pharmaceutical, industrial, biodefense and bioenergy research. Success in developing this rational approach will deliver huge reward, making the unique capabilities of the MaNDi Macromolecular Neutron Diffractometer accessible and available to 1000 s of structural molecular biologists.

\section{Mission Relevance}

The unique capabilities of the MaNDi instrument at Spallation Neutron Source should have key and strategic impact on development of bioinspired materials and systems for the solar, hydrogen, bioenergy and nuclear programs of interest to DOE. In particular, this research will 
deliver new strategic capabilities in neutron protein crystallography at ORNL that will allow us to target cellular, signaling, and membrane-bound proteins and enzymes of interest in medical, pharmaceutical, industrial, biodefense and bioenergy research. The ability to target and analyze cellular, signaling and membrane-bound proteins and enzymes of interest to health, pharmaceutical and industrial research will be of broad and specific interest to many individual researchers working in other government, industrial or academic laboratories on projects of interest to core National Science Foundation and National Institutes of Health programs.

\section{Results and Accomplishments}

We are developing this work in two phases: the first to develop protocols and the second to apply them to proteins of biological interest. Specifically, in phase 1, we survey well behaved and well known protein systems to determine whether we can generalize the critical biochemical parameters required to seamlessly transition from $\mathrm{H}_{2} \mathrm{O}$ - to $\mathrm{D}_{2} \mathrm{O}$-based solutions. This knowledge will enable the volume optimization to be carried out using a minimal sample. In phase 2 , demonstration projects will provide paradigm models for key classes of enzymes and proteins involved in health, medicine, biocatalysis, and processing, including DOE's biofuels, hydrogen production, and solar initiatives, as well of proteins of key fundamental and biomedical importance. The improved crystallization and neutron analysis of any one of these systems would be a significant achievement in itself, not only providing new and enhanced structural information, but also elucidating the mechanism and understanding of action. In FY 2007, we initiated work on 24 different proteins at ORNL. Proteins were selected from functional classes of proteins, targeting proteins where the X-ray structures are known, and that are amenable to neutron diffraction, and that crystallize easily, and where the neutron structure are of interest in understanding aspects of its biological role and function. In total, 39 protein samples (some duplicates from different preparative batches) have been included in initial crystallizationscreening experiments, and each has been surveyed against 1,536 screening experiments. This corresponds to 59,904 experiments performed.

\section{3: Magnetic Structure under Simultaneous High-Temperature/ Ultra-High-Pressure Conditions}

Chris A. Tulk, Antonio Moreira Dos Santos, Jamie Molaison, Bryan Chakoumakos, Juske Horita, and Dave Cole.

\section{Project Description}

This project will develop high-pressure and high-temperature capabilities and couple these with SNS-SNAP high-pressure Paris-Edinburgh cells in order to study properties of magnetic materials. The technical challenges of conducting simultaneous high-pressure and hightemperature neutron scattering experiments have been stymied by the need for large sample sizes and the lack of suitable large volume pressure devices that allow wide-angle neutron diffraction. This situation is rapidly changing as new high-pressure technologies are being developed nearly concurrently with neutron diffraction instrumentation. As a result, it is now highly feasible to 
combine high-pressure, high-temperature neutron instrumentation suitable for magnetic studies, such that measurements are possible under these multiple extreme environmental conditions (a developing trend in materials research). Candidate materials include many of the binary metal oxides such as $\mathrm{MnO}, \mathrm{FeO}$ (i.e., the rhombohedral distortion), $\mathrm{CoO}$, and $\alpha-\mathrm{Fe}_{2} \mathrm{O}_{3}$.

\section{Mission Relevance}

In this project, we are exploring the behavior of magnetic materials at high pressures by neutron diffraction, so it naturally falls under one of the major research areas of ORNL, namely neutron science. This research also fits into DOE's broader mission to operate world-class neutron scattering facilities and the Materials Science and Technology subprogram within the DOE Office of Science Basic Energy Sciences program. This project focuses on providing a fundamental understanding of the magnetic properties of materials under extreme conditions, and we fully expect these results to be relevant to understanding complex magnetic interaction in applied systems. More specifically, the high-pressure sample environments developed for this project will provide unprecedented pressure ranges for doing neutron diffraction in the United States. This project will provide tools that will benefit the Spallation Neutron Source and the High Flux Isotope Reactor (HFIR) user facilities.

\section{Results and Accomplishments}

By far the most challenging activity completed during FY 2007 was the design, fabrication, and assembly of the microfurnace components. The design was specifically adapted to the design details of the new style Paris-Edinburgh VX-5 series high-pressure device. Several tests were conducted offline to increase our understanding of pressure cell behavior at high temperature, to optimize the design performance of the furnace system, and to perform safety checks of the integrated system components. We conducted single-crystal studies of very small samples embedded in an indium pressure medium and tests of $\mathrm{Mn}_{3} \mathrm{O}_{4}$ magnetic samples in tungsten carbide (WC) conical anvils with Fluorinert pressure medium to evaluate sample behavior at the highest possible pressure (using WC anvils) up to $100 \mathrm{kbar}$. These studies were done before conducting room-temperature experiments on the same compounds on the WAND instrument at HFIR. We also tested the new alumina and single-crystal sapphire conical gaskets in new conical WC anvils as part of our search for a suitable gasket material for the graphite furnace. We also assembled and tested a new automated pumping system that is to be integrated with the highpressure cell for future use. 


\section{5: A Helicon Ion Source for the Spallation Neutron Source}

Robert F. Welton, F. Wallace Baity, and Richard H. Goulding

\section{Project Description}

The overall scientific capability of the Spallation Neutron Source (SNS) can be greatly enhanced by increasing neutron production. One approach to increasing neutron production is making available proton beams of greater intensity and reliability than can be presently achieved using conventional ion sources. The goal of this project is to develop a new type of $\mathrm{H}$-ion source using a helicon-generated plasma closely coupled to the state-of-the-art SNS H-ion source. The helicon hydrogen plasma generator, recently developed by the ORNL Fusion Energy Division, has the capability to produce plasmas 10 times more dense than today's H-sources using about one tenth the RF power. If successful, this project will provide a prototype ion source for SNS operations as well as future upgrade projects and advance the state of the art for positive and negative ion sources. The approach would be applicable to other accelerators and may also allow the high power RF plasma generators now being developed for use in ITER's neutral beam injectors to be replaced with higher-reliability helicon generators.

\section{Mission Relevance}

The development of reliable neutron production is critical to the success of the SNS and future power upgrade projects. Consequently, this project, if successful, will greatly benefit the DOE Office of Science. In addition, the development of a high-brightness, multipurpose plasma ion source capable of achieving significantly higher beam currents, duty-factor, and lifetime than currently available will benefit many areas of science and technology, including (1) future highpower accelerator facilities, (2) industrial applications such as ion implanters and neutron sources, and (3) H-ion sources for high-energy neutral beam injectors for fusion devices such as ITER.

\section{Results and Accomplishments}

All of the FY 2007 goals from the original proposal were met by the end of the fiscal year. The helicon plasma generator used in previous experiments was restarted, and similar plasma densities of up to $10^{13} \mathrm{~cm}^{-3}$ were achieved at power levels of $1.5 \mathrm{~kW}$ and frequencies of 13.56 and $21 \mathrm{MHz}$.

\section{Publications}

Welton, R. F., M. P. Stockli, S. N. Murray, Jr., J. Carr, Jr., and J. R. Carmichael. 2006. “A Proposed Helicon Driver for the SNS Ion Source," Proceedings of the International Linear Accelerator Conference, Knoxville, Tenn., USA, p. 367.

Goulding, R. H., R. F. Welton, F. W. Baity, and D. O. Sparks. 2007. "A Helicon Ion Source for the SNS." Proceedings of the Topical Conference on Radio Frequency Power in Plasmas, Clearwater, Florida, USA. 



\section{7: Biomass Ethanol from Clostridium thermocellum: A Systems Biology Analysis}

Jonathan Mielenz, Babu Raman, Catherine McKeown, Miguel Rodriguez, Jr., Steven Brown, Patricia Lankford, Chongle Pan, Gregory Hurst, and Nagiza Samatova

\section{Project Description}

Technology to produce ethanol from lignocellulosic biomass has been known for years, but the process costs and efficiency have limited production. A game-changing microbe is the anaerobic thermophilic bacterium Clostridium thermocellum, which ferments cellulose directly to ethanol and other metabolic products using its multi-enzyme cellulase complex, called a cellulosome, which eliminates the need for addition of cellulase enzymes. This combining of unit operations (i.e., fermentation and cellulase production) is called consolidated bioprocessing and can significantly reduce the cost of ethanol from cellulose-containing material such as plant biomass. However, there is a dearth of information regarding the basic metabolism of $C$. thermocellum that is needed for further development of ethanol production. In this study, we used microarray technology to probe the genetic expression of $C$. thermocellum ATCC 27405 during cellulose and cellobiose fermentation. In addition, an analysis of the proteins expressed during these fermentations was completed using both qualitative and quantitative tandem mass spectrometry (MS) data, with heavy N15 metabolic labeling for quantization. Levels of known fermentation by-products including ethanol, lactic acid, and acetic acid were determined during the fermentation. Finally, as an extension beyond the original LDRD goals, growth, fermentation byproducts, and cellulosomal proteins present when C.thermocellum was grown on alternate carbohydrates were evaluated.

\section{Mission Relevance}

The DOE Office of Biomass Program (OBP) has actively supported biomass ethanol technology R, D\&D for over 25 years. Their multiyear program plan included such work within the Advanced Technology Development Section. Technical management at OBP believes that this project makes sense regarding their mission. In addition, the concept of consolidated bioprocessing, which this fermentation system represents, has been emphasized as an important bioengineering concept by senior management in the Office of Science. The primary outcome of this research will be building a stronger foundation for lower cost biomass ethanol fermentation, thus improving national security, our environment, and our rural economy. In addition, this research will directly support the reduction in the cost of biomass ethanol needed to meet the President's goal of replacing 20\% of this country's gasoline use with biofuels by 2017.

\section{Results and Accomplishments}

This work has accomplished the project goals to study cellulose fermentation using systems biology approach based on integrative analysis of transcriptomic, proteomic, and metabolomic data. The impact of fermentation of mixed carbohydrate substrates has been also evaluated. 
Using the $C$. thermocellum microarray developed in year one, the genes expressed during timecourse cellulose fermentations were determined. Gene clusters with similar temporal patterns of expression were created. Broadly, genes involved in energy production, translation, glycolysis, and amino acid, nucleotide, and coenzyme metabolism displayed a progressively decreasing trend in gene expression as the batch fermentation proceeded. In comparison, genes involved in cell structure and motility, chemotaxis, signal transduction, and transcription and cellulosomal genes showed an increasing trend in gene expression. Essentially, during cellulose fermentation, the capacity of $C$. thermocellum to sense and respond to its environment increases and cells become more motile over time.

We also used multidimensional LC-MS/MS technology and 15N-metabolic labeling strategy to quantify changes in cellulosomal proteins in response to various carbon sources (cellobiose, crystalline cellulose (avicel), amorphous cellulose, and combinations of avicel, pectin, and xylan). Proteomic analysis identified over 50 dockerin- and 6 cohesin-module containing components, including 20 new subunits. The list included several proteins of potential interest which specifically respond to the presence of "non-avicel" substrates in the culture medium. Overall, the transcriptomic and proteomic results suggest a well-coordinated temporal and substrate-specific regulation of cellulosomal composition in $C$. thermocellum. These findings enhance our current understanding of $C$. thermocellum physiology and lay the foundation for future studies with natural biomass substrates. Future research will be continued as part of the BioEnergy Science Center with priority on natural biomass substrates.

\title{
Publications
}

Brown, S. D., B. Raman, C. K. McKeown, S. K. Kale, Z. He, and J. R. Mielenz. 2007. "Construction and Evaluation of a Clostridium thermocellum 27405 Whole-Genome Oligonucleotide Microarray," Appl. Biochem. Biotechnol. 136-140, 663-67 .

Brown, S. D., et al. 2007. "Construction and Evaluation of a Clostridium thermocellum ATCC 27405 Whole-Genome Oligonucleotide Microarray.” Appl. Biochem. Biotechnol. 137, 663-674.

\section{8: A Model System for Analyzing Whole-Body Toxicity of TICs, TIMs, and Chemical Warfare Agents}

\author{
Brynn H. Voy, Elissa J. Chesler, Jennifer Sedowski, and Wayne H. Griest
}

\section{Project Description}

There is great concern by the United States military and Homeland Security organizations that unguarded stockpiles of industrial process chemicals can be used as highly effective chemical warfare agents. An example is methyl isocyanate (MIC), which killed and injured thousands following its accidental release into the environment at a Bhopal, India, Union Carbide plant in 1984. The extent of toxicity among Bhopal survivors varied widely across the exposed population; part of the variation was attributed to genetic susceptibility. The Bhopal accident is one of countless examples of how an individual's genetic background impacts his/her response 
to environmental factors. Our goal is to establish ORNL expertise in systems biology approaches to gene-environment interactions, while taking advantage of mouse genetic reference populations maintained at ORNL. Environmental factors include a diverse spectrum of potential exposure agents; we have chosen MIC because of its potential relevance to Homeland Security. The genetic reference population in use is a panel of BXD (C57BL/6J X DBA/2J) recombinant inbred (RI) strains. Our strategy for this project is to identify phenotypic traits that respond to MIC in a predictable manner and then use these measures as the basis for a survey of MIC response across a genetically diverse BXD RI strain population. Integration of these meaningful physiological endpoints with gene expression profiling in a relevant target tissue and with existing genotype data forms the basis for our strategy to identify molecular networks of MIC response and genetic susceptibility.

\section{Mission Relevance}

The long-term benefit to military and Homeland Security sponsors will be twofold: first, we can identify genetic variants that will tend to make individual soldiers particularly sensitive to chemical exposures, and, second, we can point the way to molecular mediators of response that might make good therapeutic targets. The military has several programs in this area, such as the United States Army Medical Research Institute of Chemical Defense. The National Institutes of Health (NIH) has also developed programmatic interest in this area, implemented as the NIH Countermeasures against Chemical Threats (CounterACT) Research Network. In a more general sense, the results from using BXD strains as a model for gene-environment interactions can serve as preliminary data for proposals targeted to the Genes and Environment Initiative recently launched by the National Institute of Environmental Health Sciences (NIEHS).

\section{Results and Accomplishments}

In the second year of this project, we expanded the number of recombinant inbred mouse strains to cast a wider net of genetic variation and tested the utility of plasma cytokines as indices of MIC exposure. We found that this expanded panel of mice exhibited a consistent hematological response to MIC, including fluid loss and concentration of blood volume and a reduction in core body temperature. Both responses were quickly corrected by homeostatic mechanisms, with values near unexposed control mice by 24 hours after exposure. However, heritability estimates did not support significant genetic variation in the response to MIC. A trend for differential magnitude of the MIC response for some plasma analytes was suggested, but the heritable variation was deemed insufficient for the systems genetics approach originally conceived. Plasma cytokine levels (a panel of 22/mouse) assayed from a subset of strains also did not support heritable differences in the MIC response, although we did identify significant variation in baseline levels of select cytokines (e.g., Interleukin-6) that will be informative for other ongoing studies. In retrospect, mice did not exhibit the genetic variation in the response to MIC that was critical to this project, despite the fact that we profiled a battery of physiological measurements that clearly reflected MIC exposure. It is possible that at even lower MIC doses than those tested (primarily LD5, LD10, and LD20), heritable differences could be detected, although they would not necessarily be associated with adverse physiological effects. It is also possible that the BXD strain panel, containing only 2 allelic possibilities for each polymorphism does not contain MIC risk alleles that may exist in a larger population. 


\section{3: Systems Biology of the Mammalian Cilium: A Cellular Organelle Essential for Human Health and Development}

Edward J. Michaud

\section{Project Description}

Primary cilia are microtubule-based organelles that project from the surface of cells in organisms as diverse as green algae and humans. Primary cilia function as biochemical and mechanical sensors for the cell, receiving information from neighboring cells and from the environment. Cilia play critical roles in human development and physiology, as evident from numerous genetic disease syndromes arising from defects in cilia. Symptoms of these diseases include abnormalities in left-right sidedness, retinal degeneration, hydrocephaly, infertility, obesity, respiratory distress, and cystic lesions in the kidney, liver and pancreas. Recent comparative genomics and proteomics studies revealed that the primary cilium is composed of about 1000 proteins, but most of their functions are unknown. We are using Caenorhabditis elegans (worms) and Mus musculus (mice) as model organisms to determine the biological functions and interactions of highly conserved cilia proteins. The major focus of this project is on generating mutations in orthologous mouse cilia genes with a state-of-the-art, high-throughput, and costeffective mutagenesis strategy developed at ORNL. The phenotypes of mutant mice will be examined at the molecular, cellular, and whole-animal levels, which will provide insights into the assembly and function of cilia. This work may also facilitate the development of new methods for the diagnosis, treatment, and prevention of cilia diseases.

\section{Mission Relevance}

The DOE initiated the Human Genome Project and played a major role in the completion of the human genome sequence. DOE, through the Joint Genome Institute, continues to provide the scientific community with DNA sequences of many other prokaryotic and eukaryotic organisms. These sequenced genomes are facilitating innumerable comparative genomics and proteomics approaches, including the identification of the "cilia proteome," which would otherwise have been impossible without these sequences. This project will help annotate the conserved cilia proteome in humans by initiating a systems-biology-based understanding of cilia assembly and function through mutagenesis of the homologous genes in mice. This work also benefits the National Institutes of Health (NIH). Cilia dysfunction causes human diseases, including Meckel Syndrome, Kartagener's Syndrome, Polycyctic Kidney Disease, Primary Ciliary Dyskinesia, Nephronophthisis, Bardet-Biedl Syndrome, Alstroms Syndrome, Joubert Syndrome, and Orofaciodigital Syndrome. Therefore, many institutes within the NIH will be potential sources of follow-on funding, including the NCI, NEI, NIA, NIAMS, NIBIB, NICHD, NIDCR, NIDDK, NIGMS, and NINDS.

\section{Results and Accomplishments}

In FY 2007, the second year of this project, we focused primarily on generating and characterizing new mouse models of cilia disease. The goal was to determine the in vivo function of novel cilia genes by generating mice with mutations in these genes, and then characterizing the phenotypes of the mutant mice. We screened DNA and cDNA templates from 4,000 mice in 
ORNL's Cryopreserved Mutant Mouse Bank (CMMB) in order to identify mice with ethylnitrosourea (ENU)-induced point mutations in cilia genes. We completed a 36.7-Mb screen of DNA and cDNA templates from the 4,000 CMMB mice for 18 cilia genes. We identified 15 mutations in eight of these genes. Eleven of the fifteen mutations cause changes in the encoded proteins that could alter function, which include nine nonsynonymous amino acid substitutions, one premature termination codon, and one splice-donor change likely to cause exon skipping. We recovered live mice harboring three of these cilia-gene mutations by performing intracytoplasmic sperm injection with cryopreserved sperm from the CMMB mice. The three lines of mice have mutations in the genes: dynein, axonemal, heavy chain 3 (Dnahc3); echinoderm microtubule associated protein like 2 (Eml2); and transmembrane protein 67 (Tmem67). We recovered live heterozygous mice harboring each of these mutations, intercrossed the mice, and obtained homozygous mice that will now undergo phenotype analyses. We also obtained germline transmission of a gene-trap embryonic stem-cell line harboring a knockout mutation in the gene, TNF receptor-associated factor 3 interacting protein 1 (Traf3ip1). We recovered and intercrossed heterozygous mice harboring the Traf3ip1 mutation, but have not yet obtained homozygous offspring, suggesting that this mutation may result in an embryonic lethal phenotype. Phenotypic investigations of all four lines of mutant mice are under way.

\section{Publications}

Michaud III, E. J., et al. 2005. "Efficient Gene-Driven Germ-Line Point Mutagenesis of C57BL/6J Mice." BMC Genom. 6, 1-18.

Michaud III, E. J., et al. 2005. "Gli2 and Gli3 Localize to Cilia and Require the Intraflagellar Transport Protein Polaris for Processing and Function." PLoS Genetics 1, 480-488. Michaud III, E. J. and B. Yoder. 2006. "The Primary Cilium in Cell Signaling and Cancer." Cancer Research 66, 6463-6467.

\section{0: Accelerated Domestication in Populus: Harnessing the Recently Sequenced Genome for Bioenergy Crop Production}

Timothy Tschaplinski, Tongming Yin, Xiaohan Yang, Lee Gunter, Sara Jawdy, Xinye Zhang, Donald Kaczmarek, and Maud Hinchee

\section{Project Description}

Plant domestication is the conversion of a wild plant, whose morphology and physiology are directed towards survival and reproduction in nature by natural selection, into a cultivated plant whose attributes have been altered by artificial selection to increase the yield of useful products. Although many annual crops benefit from centuries of domestication efforts, perennial organisms that could play a central role in providing a renewable source of feedstock for conversion to fuels have not. The goals of this project are to identify candidate genes for accelerated domestication in Populus using a comparative genomics approach and to determine the function and potential economic value of these candidate genes via cisgenesis (modified activity of native genes). Specifically, we are (1) identifying candidate genes associated with large-effect quantitative trait loci (QTL) for dehydration tolerance in a hybrid poplar pedigree, 
(2) cloning homologs of candidate genes that are transcription factors for dehydration tolerance in other species, (3) engineering poplar candidate genes for up- and down-regulation for cisgenic insertion into a productive poplar clone in collaboration with ArborGen (Summerville, SC), (4) producing multiple lines with each cisgenic construct to create a range of phenotypic variation, and (5) validating early phenotypic expression. Our long-term goal is to produce poplar clones with increased drought tolerance to maintain high plant and stand productivity without the need for costly irrigation.

\section{Mission Relevance}

This work benefits DOE Office of Biological and Environmental Research by testing the concept of accelerated domestication and leverages Office of Science's efforts to sequence the Populus genome. Increasing dehydration tolerance of Populus will increase stand productivity, improve overall feedstock uniformity, and reduce production risk and the cost of supplying biomass feedstocks for the production of alternative fuels. Whereas, Populus is a top priority crop for domestication for DOE, similar genomic approaches for accelerated domestication will apply to herbaceous energy crops that are of interest to the U.S. Department of Agriculture. The Energy Policy Act of 2005 and the resulting National Biomass Initiative is specifically aimed at improving biomass technologies and increasing the amount of biopower, biofuels, and bioproducts used. The research will contribute to the President's goal for the nation of producing $30 \%$ of its transportation fuel from biomass. Responsible agencies include the Environmental Protection Agency, U.S. Department of Agriculture, DOE, and National Science Foundation.

\section{Results and Accomplishments}

We have created and filled gaps in a large genetic marker linkage map for poplar TxD family 822, identified QTL for dehydration tolerance, and then aligned the gene sequence with the target QTL regions (for both TxD families 822 and 331). We annotated the sequence within the marker intervals to produce a list of candidate dehydration tolerance genes for further functional analysis following transformation by ArborGen. Candidate genes that were identified from the poplar gene sequence had a high similarity match with Arabidopsis and are known drought tolerance transcription factors (TF), including DREBs, AREB1/ABF2, NAC, and myb genes. To create a range of phenotypic variation, cisgenic gene constructs with up- and down-regulated function of native candidate genes were prepared and sent to ArborGen. The transformed, putatively drought-tolerant poplar clones were produced, transported back to ORNL, and are now growing well in our greenhouse, awaiting phenotypic validation by physiological and metabolomic assessments. We conducted an extensive drought experiment on poplar, collected and prepared plant samples for global profiling of transcripts by DOE's Joint Genome Institute (JGI). The 454 and Solexa short-read sequencing of cDNA from poplar exposed to short- and long-term drought treatments are currently being conducted by JGI to identify additional candidate transcripts in the drought-responsive pathway of poplar. Ultimately, field trials of the generated cisgenic poplar clones that are potentially more drought tolerant, and hence productive, will be conducted by ArborGen to validate long-term field performance. 


\section{Publications}

Tschaplinski, T. J., et al. 2006. "Phenotypic Variation and Quantitative Trait Locus Identification for Osmotic Potential in an Interspecific Hybrid Inbred $\mathrm{F}_{2}$ Poplar Pedigree Grown in Contrasting Environments." Tree Physiol. 26, 595-604.

\section{1: Disentangling Soil Respiration Using Genomic Techniques}

Aimée T. Classen, Christopher W. Schadt, and Richard J. Norby

\section{Project Description}

Belowground respiration $\left(\mathrm{R}_{\text {Soil }}\right)$ is an integrator of ecosystem metabolism and a key component of the interaction between the terrestrial biosphere and the atmosphere. It consists of three components - root respiration, bacterial respiration, and fungal respiration. Each of these components is likely to respond to changes in the global climate differently, yet scientists have no way of adequately teasing apart root, bacterial, and fungal respiration from bulk soil respiration measurements. Our LDRD project proposed to devise a new approach for predicting the effects of changes in atmospheric $\mathrm{CO}_{2}$, atmospheric temperature, and precipitation on the integrated metabolism (as represented by $\mathrm{R}_{\text {Soil }}$ ) of a model old-field ecosystem in a manipulative, multifactor, climate change experiment. Our project sought to use quantitative real-time polymerase chain reaction (PCR) to assay portions of the ecosystem transcriptome that we hypothesized would be indicative of autotrophic (plant root) and heterotrophic (fungal, bacterial) respiratory activity. In so doing, we aimed to test the hypothesis that information expressed at the genomic and metabolic levels for evolutionarily conserved and ubiquitous genes is sufficient for estimating the ecosystem function to which such genes are coupled.

\section{Mission Relevance}

This project will directly benefit research programs in DOE's Office of Biological and Environmental Research (BER). The primary goal of the Program for Ecosystem Research (PER) is to understand, and be able to predict, the effects of environmental changes associated with energy production on the structure and functioning of terrestrial ecosystems. PER has specifically encouraged explorations into the transfer of information across levels of biological organization and has encouraged the use of genomics in ecological research. Thus, our goal of using genomic tools to understand process response and enable ecosystem monitoring is consistent with the overall goals of the DOE/BER.

\section{Results and Accomplishments}

Broadly, our research found that the combination of multiple global change factors may have important effects on microbial community structure and function. This is the first experiment that we know of to accomplish this task within such a complex experimental design and system. Specifically, our project resulted in four major accomplishments: (1) We were able to assay the microbial community in a complex soil and determine what factors had the most impact on community structure and function and which organisms we should target in future studies. (2) 
This project enabled us to establish and optimize standardization of quantitative PCR conditions for bacteria, fungi, and plant rDNA genes and bacterial citrate synthase genes in soil for the first time in natural soils. These techniques will enable ORNL to track the importance of these organismal groups in ecosystem processes such as soil respiration. (3) This project established a system for assessing how citrate synthase genes are distributed across a broad diversity of bacteria and how these bacteria are responding to changes in the global climate. (4) The molecular methods necessary to quantify citrate synthase diversity, activity, and abundance in soils were established for the first time. This project will enable ORNL scientists to begin to use molecular approaches to partition soil respiration.

\section{2: Photo-Regulated Peptide-Protein Interaction Systems for Bionanotechnology Applications}

Robert F. Standaert

\section{Project Description}

This project centers on a combined chemical and biochemical approach to developing lightresponsive peptide-protein interaction pairs which have light-tunable affinity and that can be coupled to other systems of interest. The peptide partner in each case is modified by the installation of a photoresponsive amino acid that isomerizes reversibly upon irradiation. Key challenges include the design and synthesis of the amino acids, identification of effective sites for their installation, and protein affinity measurements for photoisomeric forms of the peptides. The methodology is in principle broadly applicable, but this project is focused on two specific peptide-protein pairs selected for their versatility and ready applicability to bionanotechnology applications.

Two major long-term objectives drive the development of light-regulated interaction systems. The first is remote control of biological systems. Controlled molecular association is a fundamental process governing the structure and function of cells. Tools that enable the extracellular manipulation of molecular association within cells will enable both the dissection of complex signaling pathways and the noninvasive manipulation of cell functions, with applications ranging from basic biology to tissue engineering. The second is light-directed patterning and assembly of peptide- and protein-based materials under biocompatible, aqueous conditions. The use of light to regulate the assembly of these materials would provide exquisite spatial resolution as well as precise temporal control and the ability to create dynamic structures that can be reconfigured in real time for diverse applications.

\section{Mission Relevance}

This work is relevant to a number of programs within DOE and other major federal agencies. The methods being developed are particularly well suited for the Genomics:GTL program (DOE, Office of Biological and Environmental Research) and projects within the Center for Nanophase Materials Sciences (CNMS, sponsored by the DOE Office of Basic Energy Sciences). Our methods will have broad applicability in life science and materials research and are thus also of 
interest to other agencies, particularly the National Institutes of Health (NIH) and DoD. Major proposals to the National Cancer Institute (NCI), National Institute for Biomedical Imaging and BioEngineering (NIBIB), and Defense Threat Reduction Agency (DTRA) were submitted in FY 2006. A collaborative proposal has been funded through the National Eye Institute (5R01EY016094-02), and a Work for Others subcontract for this work is pending.

\section{Results and Accomplishments}

The proposed goals of the project focused on two tasks: the first associated with remote control of cells and the second with light-regulated materials assembly. In this project year, efforts associated with remote control of cells were shifted further toward neuronal signaling (via the neurotransmitter $\gamma$-aminobutyric acid, GABA) instead of nuclear protein transport because of more immediate promise of external funding. The ultimate goal of the GABA work is to produce photoregulated $\mathrm{GABA}_{\mathrm{C}}$ effectors that can be tethered to other molecules or surfaces. In FY 2006, we identified a novel class of $\mathrm{GABA}_{\mathrm{C}}$ antagonists (2-aminoethyl alkylphosphonates) that could potentially be used for this purpose. In FY 2007, we finalized this work and published the results. We have followed up by synthesizing new representatives of this class with substituents added to determine where there may be opportune sites for installing photoregulatory and tethering moieties. Four new compounds have been synthesized, with physiological testing pending, and synthesis of additional candidates is in progress. We have also synthesized approximately 15 additional analogs of the GABA analog muscimol. Notable among this group are six compounds incorporating photoisomerizable azobenzene moieties, the physiological evaluation of which is pending. In the area of nuclear protein transport, we have successfully implemented a fluorescence polarization-based binding assay for the nuclear import receptor subunit importin $\alpha$. However, we encountered difficulty in characterizing a photoisomerizable ligand with this assay due to apparent energy transfer between the peptide's azobenzene nucleus and the tetramethylrhodamine fluorophore, which inhibited photoisomerization and appeared to initiate side reactions. Toward the end of the project, we redirected our efforts toward peptides containing an alternative type of chromophore to replace the azobenzene.

Our efforts in light-directed materials synthesis have focused on streptavidin-binding peptides into which photoisomerizable amino acids have been incorporated. We attempted the preparation of 24 peptides as candidates for study and were successful in 17 cases. All of the failed sequences involved the combination of a particular azobenzene-containing amino acid (Aza) with the natural amino acid tryptophan, suggesting that the two are incompatible under the synthesis conditions employed. We have completed a preliminary screen of the 17 candidate peptides and have found promising indications that in at least one case, photoisomeric forms of the same peptide display different affinities for streptavidin.

\section{Publications}

Standaert, R. F. and D. S. B. Park, 2006. “Abc Amino Acids: Design, Synthesis, and Properties of New Photoelastic Amino Acids.” J. Org. Chem. 71, 7952-7966. 


\title{
00244: Analysis of the Role of miRNAs: Profiling miRNA Expression across BXD Recombinant Inbred Mouse Strains in Support of Mouse Collaborative Cross Program
}

\author{
Yisong Wang, Mitchell Klebig, Yun You, Dabney Johnson, and Elissa Chesler
}

\section{Project Description}

ORNL's mouse facility is in transition to a new research arena, in which a joint community effort will generate a large panel of recombinant inbred strains derived from a genetically diverse set of eight existing founder mice called "collaborative cross mice." Since the mechanisms that underlie disease susceptibility and progression are often influenced by numerous genetic/environmental factors, the random recombination of genetic materials of the eight different inbred strains theoretically would provide the grounds for different response to disease susceptibility and/or environmental factors, and eventually allow researchers to map candidate genes that may link to disease/environmental susceptibility. In support of this promising resource, we have rescoped our proposal to explore the established BXD (C57BL/6J x DBA/2J) mice for gene networks that regulate miRNA expression with special emphasis on the lung. MiRNAs are short 20-22 nt noncoding RNA that play important roles in gene regulation. Recent studies demonstrate that miRNAs are frequently deregulated in cancer cells. Statistics show that individuals exposed to asbestos, an inhalation fiber/hazard known to cause DNA damage and apoptosis, have five times higher risk of developing lung mesothelioma. Smokers have a 10 times higher risk, whereas individuals exposed to smoking and asbestos have a 50-90 times higher risk of developing lung cancer. The role of miRNAs in cellular susceptibility to asbestos/smoking-induced lung damage has not been explored. In this study, we have initiated a pioneer experiment to systematically map miRNA function and regulation using BXD mouse models for human lung disease, and explore miRNAs as biomarkers for the evaluation of asbestos/smoking-related lung cell transformation.

\section{Mission Relevance}

Because of the potential broader impact of miRNA on every human disease, the study is relevant to many National Institutes of Health translation medicine programs. Our results and resources generated in this project could position us at the forefront to pursue R01 and R21 funds from National Cancer Institute and to secure our funding petition for several NIH calls, such as ID\#: PAS-07-241, RFA-GM-08-001 and PAR-06-209. Data derived from this study will strengthen the justification for our long-term Collaborative Cross program/Mouse User Facility Center through, for example, the funded NIH U54 center grant, which will serve as the major operating grant for our Collaborative Cross program in replacement of our current Field Work Proposal (DOE OBS) 3 years from now. The work has also helped us obtain a Battelle COPD Bioinitiative Fund for the miRNA studies.

\section{Results and Accomplishments}

MiRNA expression profiling by miRNA microarray showed that five miRNAs are differentially expressed in the lungs of six BXD strains indicating potential strain-dependent genetic variations in the control of miRNA expression. We also found that specific clusters of miRNAs are 
commonly regulated by asbestos and cigarette smoke solution (CSS) in nontransformed lung mesothelial cells and bronchial fibroblast cells. These include two upregulated miRNAs and five downregulated miRNAs. Four of the seven commonly-regulated miRNAs have been confirmed by realtime qPCR. A group of miRNAs that shows a mutually exclusive expression pattern in cells exposed to asbestos or CSS alone was also identified by microarray analysis. We also showed that three miRNAs were significantly upregulated and four miRNAs downregulated after chrysotile exposure to a transformed lung epithelial cell line. Moreover, asbestos-induced deregulation of two miRNAs correlated with the reduction of cell growth and induction of cell death. Interestingly, exposure of staurosporine, etoposide and $\mathrm{H}_{2} \mathrm{O}_{2}$, which cause DNA damage and apoptosis through different mechanisms from asbestos, to the transformed lung epithelial cells, had no effect on the two specific miRNA expression, indicating that deregulation of those miRNAs may be asbestos-specific. We also identified an miRNA that specifically overexpressed in lung-transformed cells. Gene ontology analysis put this miRNA in NFKB and DNA checkpoint pathway that may play important roles in the synergistic effect of asbestos and smoking in lung cancer susceptibility, and that this may represent the first to demonstrate the relationship between miRNA expression and asbestos/smoke-induced lung cell transformation.

\section{Publications}

Giannone, R. J., et al. 2007. "Dual-Tagging System for the Affinity Purification of Mammalian Protein Complexes." BioTechniques 43, 296-302.

Gomez, M. V., et al. 2006. "PARP1 Is a TRF2-Associated Poly(ADP-ribose) Polymerase and Protects Eroded Telomeres." Mol. Biol. Cell. 17, 1686-1696.

Wang, Y. and Y. Liu. 2006. "Msh2 Deficiency Leads to Chromosomal Abnormalities, Centrosome Amplification, and Telomere Capping Defect." Oncogene. 25, 2531-2536.

\section{7: Novel Approaches for Uncovering Total Environmental Gene Expression Patterns}

Martin Keller, Zamin K. Yang, Amudhan Venkateswaran, Mircea Podar, and Christopher W. Schadt.

\section{Project Description}

Tremendous progress has been made in studying total transcriptional activity in microorganisms, due to the emergence of newly developed genomics-based technologies such as RT-PCR and microarrays using total RNAs. However, none of these approaches can be routinely applied for comprehensive understanding of environmental microbial communities and uncultured bacterial cells, since both methods require background genomic information to allow for design of specific primers and/or microarray probes. The purpose of this project is to circumvent this limitation by developing a method involving direct sequencing of cDNA from the environmental samples utilizing a high-throughput sequence analysis system such as Bio454. These methods will allow total transcriptome sequencing and analysis from environmental samples, unknown bacteria, or uncultured cells. We will develop these novel methods using Desulfovibrio vulgaris as model organism. Then we will study sediment samples from the Environmental Remediation Science 
Program Field Research Center site or other simple communities to determine gene expression of microorganisms present in low-biomass environments.

\section{Mission Relevance}

Our goals are to develop and demonstrate these novel methods in direct application to DOE mission areas. Microbial communities have direct relevance to DOE missions in bioenergy (e.g., selection of strains and consortia efficient in the conversion of cellulose to ethanol), environmental stewardship (e.g., reduction of uranium and other contaminants); and climate change science (e.g., understanding the storage and cycling of carbon through soils). By understanding how microorganisms respond directly to perturbations and manipulation of these environments through gene expression patterns, we hope to provide fundamental new insights into the functioning of these communities.

\section{Results and Accomplishments}

Our primary goal is to develop and implement methods for high-sensitivity, high-throughput, and high-content analysis of global gene expression patterns from environmental microbial communities or small numbers (a few cells) of uncultured bacteria. During FY 2007, our efforts focused along three primary tasks that were pursued simultaneously. In Task 1 (Optimizing methods for efficient removal of ribosomal and other structural RNAs), we adapted and tested three distinct techniques for rRNA removal. None of these techniques alone were found to be adequate for our intended end-use applications. We have now implemented and are currently optimizing a combinatorial two-step method, which in initial tests was able to remove approx $70 \%$ of ribosomal rRNAs. In Task 2 (Developing and testing methods for unbiased amplification of cDNA from low biomass/low activity environments), we have implemented a preliminary protocol based on phi29 polymerase amplification and are testing the sensitivity and bias associated with these new methods. So far this method achieves over 10,000-fold amplification of target cDNAs when combined with rRNA removal protocols in Task 1. In Task 3 (Enrichment and amplification of an uncultured thermophilic Ammonia Oxidizing Archaea [AOA] as a test for next-generation sequencers), we have completed the amplification of DNA, from as few as 1-20 cells. We will completely sequence this DNA before implementing the RNA protocols developed above as a first test case for implementation of these methods on uncultured bacterial cells and communities. 


\title{
00020: Systemic Approaches in Recombinant Zymomonas mobilis to the Regulation of Ethanol Fermentation
}

\author{
S. D. Brown, S. Yang, T. J. Tschaplinski, D. A. Pelletier, G. Hurst, Y. Yang, N. L. Engle, \\ S. Carroll, C. Pan, N. F. Samatova, and S. L. Martin
}

\section{Project Description}

Ethanol is currently blended as an oxygenate in transportation fuel. Its expanded use has great potential to reduce fuel imports, to create American jobs, and to improve our nation's energy security. However, there are barriers and challenges to a rapid expansion of cellulosic-ethanol production. Robust process-tolerant and inhibitor-resistant microbes are recognized as key shortterm technological goals. We are elucidating the molecular basis of process inhibitor tolerance and stress responses in Zymomonas mobilis using systems biology tools, the recently completed genome sequence, a recombinant capable of fermenting C-5 and C-6 sugars, an inhibitor-tolerant strain, and physiological studies. Understanding the molecular basis for inhibitor tolerance and stress in a genetically tractable organism will provide rapid and fundamental insight into important fermentation attributes and potential improvements for applied ethanol production. This study will provide baseline data useful for other opportunities involving modeling and simulating microbial systems, be applicable to other stressors, and have broader implications to other bioenergy-related microbes.

\section{Mission Relevance}

The Z. mobilis stress responses to be characterized in this project are aligned with key science research milestones outlined in the joint DOE Office of Science and Office of Energy Efficiency and Renewable Energy Biomass to Bioethanol roadmap report. Our systems biology approach will delineate important loci, regulators, and key metabolites in stress response and inhibitor tolerance pathways. These studies will likely lead to identification of useful biomarkers and targets for strain improvement and potentially provide broader insight into other ethanolproducing microorganisms. The study will demonstrate and expand ORNL's expertise in the application of a systems-level approach to the study of Z. mobilis, which could be readily adapted to other ethanologenic microorganisms of importance to the DOE Office of Science Genomics: GTL program and Energy Efficiency and Renewable Energy Office of the Biomass programs. The fundamental and applied outcomes of our research will assist DOE with the goals outlined in the President's Advanced Energy Initiative to help break America's dependence on foreign sources of energy and develop cleaner, cheaper, and more reliable alternative energy sources.

\section{Results and Accomplishments}

In an initial study, we elucidated the dynamics of $Z$. mobilis oxygen stress responses by characterizing transcriptomic and metabolomic profiles of aerobic and anaerobic fermentations using whole-genome microarray analysis, gas chromatography-mass spectrometry (GC-MS), and controlled cultivation. Transcriptomic and metabolomic data were consistent with faster glucose consumption and greater ethanol production under anaerobic conditions, suggested gene targets for deletion, and improved fermentation. These studies also help identify transcripts for 
genes not predicted in the primary annotation of the organism's genome and enhance our current understanding of Z. mobilis physiology.

In another set of experiments, we have identified a set of $Z$. mobilis loci for inhibitor tolerance and are now defining those that are most important for conferring tolerance to a process inhibitor. In this study, we resequenced the genome of a classically derived strain and using a combination of expression, quantitative proteomic and traditional approaches are implementing a new discovery paradigm to identify rapidly the important loci. Our approach will be incorporated into future BioEnergy Science Center research activities and advance DOE missions.

\section{1: Unraveling the Regulatory and Biosynthetic Genes that Control Cellulose Production in the Model Bioenergy Crop, Populus}

Udaya Kalluri, Timothy McKnight, Dale Pelletier, Jennifer Morrell-Falvey, Gregory Hurst, David Weston, Sara Jawdy, Linda Foote, and Lee Gunter

\section{Project Description}

The percentage of usable substrate per unit of feedstock biomass is one of the prime factors affecting efficiency of conversion to ethanol. The long-term goal of this project is to unravel novel biosynthetic and regulatory genes that control plant cell wall composition, specifically, cellulose in Populus. A twofold approach is proposed toward this goal. Task 1 is to develop a carbon nanofiber-based transient expression system to rapidly screen the roles of candidate genes in wall biosynthesis. Development of such a system will circumvent the current impediment of long turnaround times needed to generate stable transgenic plants for evaluation of their gene function in Populus. Task 2 is to develop and apply protein interaction assays, such as chromatin immunoprecipitation and pull-down assays, to cell-wall biosynthesis studies. Progress made through this project will add new technological and scientific capabilities to ORNL, furthering a comprehensive understanding of cellulose biosynthetic pathway in bioenergy crops as well as the understanding of plant systems biology-based solutions in support of other DOE missions.

\section{Mission Relevance}

A better understanding of the molecular controls on plant cell-wall composition will lead to applications to improve the efficiency of biomass conversion to ethanol. The protocols being developed through the present LDRD project are directly applicable to current and future projects sponsored by Office of Science, Basic Energy Research, such as Plant Feedstock Genomics for Bioenergy and large DOE-sponsored bioenergy research efforts, specifically, the BioEnergy Science Center (BESC). Moreover, owing to the potential broad applicability of the approaches developed through this project, we anticipate future funding opportunities to exist with DOE (scientific focus areas such as GTL: Fundamental Science), DOE-USDA, and National Science Foundation (e.g., Plant Genome Research program). These approaches will also be relevant to the anticipated DOE large science solicitations in the areas of GTL: Bioremediation and GTL: Carbon Cycling. 


\section{Results and Accomplishments}

As a first step towards our primary goal in Task 1, we sought to develop an efficient protoplast isolation protocol for Populus. We were able to successfully isolate viable protoplasts from Populus as well as Arabidopsis and switchgrass tissues. Prolonged cell viability was successfully achieved by empirical testing of several different culture compositions. A fluorescein diacetatebased protoplast viability test was used to assess viability of cells over time. Impalement and postimpalement survival of cells was improved significantly. The wall formation assay conditions have also been successfully standardized.

As a first step towards our goal in Task 2, we sought to develop protein isolation methods for Populus. We have successfully standardized both high and low molecular weight total protein extraction methods. Based on literature searches, we identified two candidate genes for initial evaluations. We have designed gene-specific primers, prepared a Populus xylem cDNA library, and amplified full-length cDNAs. Efforts are presently under way to carry out pull-down assays. Sequence information has been sent to a commercial vendor to raise monoclonal antibodies. Furthermore, total protein extracts from Populus xylem tissue were successfully employed in proteomics profiling using tandem mass spectrometry.

In summary, progress made through this proof-of-principle study will lead to breakthroughs in understanding of cell-wall biosynthetic pathways in bioenergy crops and in plant systems biology-based solutions.

\section{3: Three-Dimensional Aberration Corrected Scanning Transmission Electron Microscopy for Studying Microbiological Systems}

Niels de Jonge

\section{Project Description}

Aberration corrected 3-dimensional (3D) scanning transmission electron microscopy (STEM) is capable of high-resolution 3D imaging of specimens without a tilt stage. In a manner similar to confocal light microscopy, the sample is scanned layer by layer by changing the objective lens focus so that a focal series is recorded. Optimized 3D STEM is expected to exhibit significant advantages over tilt-series transmission electron microscopy (TEM) for conventional thin sections, such as (1) better resolution, (2) absence of mechanical tilt, (3) the capability of imaging large area thin sections, and (4) faster 3D data collection. Our goals are to fully understand the image formation mechanisms, to evaluate the feasibility of 3D STEM with respect to radiation damage, and to optimize the performance of 3D STEM for the imaging of thin sections of biomedical relevance. 


\section{Mission Relevance}

Three-dimensional (3D) STEM can be used to study the complex organization of the nanometersized assemblies of macromolecules and compartments (e.g., ribosomes, proteasomes, Golgi apparatus, and mitochondria) within Eukaryotic cells. Understanding how these structures are organized, and thereby function, within the crowded 3D volume of the cell can be applied, for example, to aid the development of new therapeutics, or improve existing ones. By establishing this new structural analysis tool and demonstrating its power on relevant biological samples we expect to be in a good position to (1) support the Genomics: GTL program and initiatives in bioenergy (e.g. by setting up a user facility), (2) pursue applications to the NIH and (3) pursue National Center for Research Resources funding including 3D STEM for biology.

\section{Results and Accomplishments}

Recently, we have obtained 3D images of conventional thin sections containing 3T3 cells showing a lateral resolution of $0.6 \mathrm{~nm}$ (the grains of the stain are resolved) and an axial resolution of $60 \mathrm{~nm}$. Conventional thin sections containing mammalian cells (NIH 3T3, mouse fibroblast cell line) were prepared at the National Institute of Health (NIH). The 3T3 cells were fixed with glutaraldehyde, stained with reduced osmium, embedded in epoxy resin and contrasted with lead. The $0.15 \mu \mathrm{m}$ thin section was imaged with a STEM (JEOL $2200 \mathrm{FS} 200 \mathrm{kV}$ $\mathrm{STEM} / \mathrm{TEM}$ equipped with CEOS aberration corrector) with $\alpha=26.5 \mathrm{mrad}$. The section was searched for a Golgi stack and at its position a focal series with 50 images was recorded at a magnification of $500 \mathrm{~K}, 512 \times 512$ pixels per slice, a lateral pixel size of $0.55 \mathrm{~nm}$ and a vertical step size of $5 \mathrm{~nm}$. The values of the lateral- and vertical resolution were determined on a grain of the stain by taking line-scans in both lateral and axial direction. The same thin section was used to image microtubules contained in spindles of the mitosis process. The sample turned out to be too density stained-leading to a strongly varying background-to determine the resolution on individual grains. As alternative measure for the axial resolution we have computed the standard deviation of the signal in both rectangles for all images in the stack. We have also developed a theoretical model describing the electron dose limited resolution of 3D STEM.

\section{Publications}

de Jonge, N., R. Sougrat, D.B Peckys, A.R. Lupini, and S.J., Pennycook. 2007. "ThreeDimensional Aberration-Corrected Scanning Transmission Electron Microscopy for Biology," in Nanotechnology in Biology and Medicine, T. Vo-Dinh, ed., CRC Press. 


\section{0: Electricity and Biohydrogen Production via a Systems-Level Understanding of Microbial Fuel Cells}

Abhijeet P. Borole

\section{Project Description}

Microbial fuel cells (MFCs) capable of generating electricity were designed, constructed, and demonstrated. The specific objective was to investigate operation of MFCs at low $\mathrm{pH}$ and to allow improvements in power output via reduction of proton transfer resistance. This was accomplished by either using low $\mathrm{pH}$ in the MFCs or by improvement of MFC designs. The ability of a heterotrophic acidophile, Acidiphilium cryptum to catalyse electricity production from glucose at $\mathrm{pH}$ in the range 2 to 5 was studied. This organism was found to conduct electrons using iron and phenosafranin as a mediator. The maximum power density achieved was $13 \mathrm{~mW} / \mathrm{m}^{2}$, which is comparable to those reported for other organisms (e.g., Shewanella, Geobacter) in a two-chamber MFC configuration. A low-pH biocatalyst was also investigated for the cathode side (Acidithiobacillus ferrooxidans) to develop a completely biocatalytic (Ptfree) low-pH MFC. In order to identify microorganisms capable of direct electron transfer at low $\mathrm{pH}$, an enrichment of organisms from an anaerobic digestor was initiated in MFCs. For comparison with neutral $\mathrm{pH}$ conditions, similar MFC was started at $\mathrm{pH}$ 7.0. The power density obtained in thepH 7.0 MFCs was $253 \mathrm{~W} / \mathrm{m}^{3}$, or $3,210 \mathrm{~mW} / \mathrm{m}^{2}$, which is close to the highest reported in the literature. Further improvement in design of MFCs via electrochemical impedence spectroscopy analysis and microbial characterization of the consortia is under way.

\section{Mission Relevance}

The project addresses DOE mission to obtain energy independence, while improving environmental quality. The project deals with energy production in the form of electricity from renewable resources such as sugars and organic acids as well as waste materials. The ability to effectively produce electricity from cheap renewable resources is an important goal for DOE. Specific programs in DOE likely to benefit from this research are the Fuel Cell and Hydrogen Infrastructure Program, Energy Efficiency and Renewable Energy, Basic Energy Sciences Genomics-GTL, and potentially Environmental Protection Agency. DoD needs remote and portable energy sources to power transmitters, receivers, sensors, monitors, etc. MFCs are very stable, low-energy devices, which could be used in such applications. This project can potentially help improve power yield from MFCs to enable use in other DoD applications requiring greater energy needs.

\section{Results and Accomplishments}

Accomplishments over the past year include (1) Design of traditional and novel MFCs with reduced ohmic resistance and compact design (also suitable for stacks). Materials and electron mediator issues associated with operating MFC's at low $\mathrm{pH}$ conditions were solved. (2) Electricity production was demonstrated in a low-pH. A. cryptum-based MFC, and we identified a mode of electron transfer. (3) We demonstrated ability to design and operate low- and neutral$\mathrm{pH}$ single organism (A. cryptum, Shewanella) or microbial consortia-based MFCs to achieve the highest power densities reported in literature. Biochemical and electrochemical characterization 
of the MFCs has been initiated. Electrochemical impedance spectroscopy is a technique that provides a blueprint of the internal resistances in a fuel cell. This will enable minimization of resistances, component by component, to achive higher power densities. (4) A collaboration with the AtlanTICC Alliance partners, Georgia Tech (GT) and Imperial College, London (ICL) has been initiated during the past few months. The PI visited with ICL and hosted GT researchers in April and October 2007. Microbial commmunity-based MFCs developed at ORNL will be characterized via 16S RNA analysis, and the MFCs will be explored for potential application development in ethanol biorefineries and in the food industry. Patents and inventions have resulted from this research.

\title{
00043: Microfluidic Platform for Individual Microbe Capture, Cultivation, and Selective Release
}

\author{
Martin Keller, Anthony Palumbo, Mircea Podar, Tim McKnight, Nance Ericson, and Mitch \\ Doktycz
}

\section{Project Description}

The recent application of molecular phylogeny to environmental samples has resulted in the discovery of an abundance of unique and previously unrecognized microorganisms. The vast majority of this microbial diversity has proved refractory to cultivation with traditional methods; thus, little is know of their physiology or capabilities. We are (1) developing a microfluidic platform which will allow the site-specific capture of individual bacteria, cultivation with controlled doubling cycles, and selective release for downstream collection and further analysis and (2) applying flow cytometry to isolation and subsequent manual cultivation of single cells. The microfluidic platform will incorporate encapsulation of cells in gel microdroplets. This cultivation under low nutrient flux conditions will be followed by further characterization and analysis of microbial microcolonies through "lab-on-a-chip" concepts. The subsequent analysis will include the implementation of multiple displacement amplification (a linear type of DNA amplification) for subsequent analysis by genome sequencing. This approach will ultimately replace the flow cytometry approach, leading to higher throughput and reduced costs. The flow cytometry approach is being further developed by applying the method to anaerobic bacteria. We are exploring methods to handle anaerobic bacteria in the flow cytometry.

\section{Mission Relevance}

The ability to grow and study previously uncultured organisms will enhance our understanding of microbial physiology and metabolic adaptation and will provide new sources of microbial metabolites. These capabilities are applicable to DOE programs involved in bioenergy, bioremediation, and carbon sequestration. In all these applications use of novel microorganisms may lead to more efficient processes relevant to DOE missions. For example, DOE is interested in isolation of more efficient bacteria for the degradation of cellulose to sugars and the processing of sugars to alcohol. In addition these capabilities will have applications to National Institutes of Health (NIH) and potentially the Department of Homeland Security (DHS). NIH 
applications could come from areas such as isolation of novel pathogens and characterization of gut microorganisms.

\section{Results and Accomplishments}

We have used the flow cytometry approach to isolate novel bacteria from groundwater samples at the DOE field research center at ORNL. These bacteria were sorted into liquid culture media and isolated from a low $\mathrm{pH}$ site where no previous isolates were obtained. We have also modified the sorting chamber to hold a standard agar plate so that bacteria that prefer growth on solid media can be isolated. We also tested and demonstrated the survival after sorting of bluegreen algae (the dominate carbon fixing organisms in the ocean) in the flow cytometer, and sorting of poplar chloroplasts for study of effects of genetic changes in poplar that could result in better growth characteristics for biofuels production.

Rapid prototyping approaches have been developed and implemented to provide fast turnaround and in-house replica production of microfluidic structures capable of generating segmented flow and alginate encapsulation of microbial populations. Using these platforms, we have successfully generated and cultured GFP-expressing $E$. coli in alginate microbeads ranging from approximately 20 to $100 \mu \mathrm{m}$ in diameter, uniformly sized dependent upon the flow rate in the bead generation section of the fluidic platform. A downstream cultivation chamber has also been implemented, which provides containment and media exchange of upwards to 1,000,000 microbeads per device. The cultivation chamber provides visual inspection of microbial growth within the alginate microbeads in a single-layer, arrayed-bead format. We will continue with implementation of downstream processing of the cells.

\section{4: Methodological Development of Computer Simulation in Molecular Biophysics}

Jeremy Smith

\section{Project Description}

Motions in proteins play a key role in their function. Here we establish a program developing methodological aspects of combining computer simulation with neutron scattering experiments with a view to characterizing correlated dynamics in proteins relevant to bioenergy in different functional states and environments. Work is also being performed aimed at understanding the functional mechanisms of cellulase activity.

\section{Mission Relevance}

Progress made through this project will place ORNL in an advantageous position to secure funding from anticipated DOE Office of Sciences, Office of Biological and Environmental Research, and the Office of Basic Energy Sciences solicitations over the next several years. The results from the proposed work will, moreover, add new technological as well as scientific 
capabilities to ORNL, which in turn will strengthen ORNL's position as a leader in biophysical bioenergy research.

\section{Results and Accomplishments}

An extensive study has been performed of the relationship between the structure and dynamics of methyl groups in proteins. Work on peptide solvation resulted in the discovery of a new driving force in peptide folding, associated with dehydration of exposed hydrophobic surfaces. The discovery of this new driving force has already had a major impact in the protein-folding research community and beyond. We have also established a computational breakthrough in the first-ever atomic-detail calculation of the vibrations in a protein crystal, and this work leads to specific predictions that will be able to be tested with instruments coming on line at the Spallation Neutron Source. Finally, work on pressure-temperature molecular dynamics has revealed the fluctuating nature of picosecond-timescale protein energy landscapes.

\section{Publications}

De Hatten, X., et al. 2007. "Force-Field Development and Molecular Dynamics Simulations of Ferrocene-Peptide Conjugates as a Scaffold for Hydrogenase Mimics." Chem. Eur. J. 13, 8139-8152.

Meinhold, L., et al. 2007. "Picosecond Fluctuating Protein Energy Landscape Mapped by Pressure Temperature Molecular Dynamics Simulation.” Proc. Natl. Acad. Sci. USA. 104, 17261-17265.

Nutt, D. and J. C. Smith. 2007. "Choosing an Appropriate Water Model for use in Biomolecular Simulations." In Physics and Chemistry of Ice. Ed: Werner Kuhs, pp.451-458. ISBN: 978-0-85404-350-7 


\section{1: Development of a Global Biogeochemistry Capability for Enhanced Climate Simulation and Earth System Modeling}

Wilfred Post, John Drake, David Erickson, Forrest Hoffman, Anthony King, Qing Liu, and Daniel Riccuito

\section{Project Description}

The objective of this project is to develop a quantitatively rigorous method for initialization of biogeochemistry model components for coupled carbon cycle-climate simulation models that employ parameter and initial condition estimation using optimization techniques and efficient data-assimilation algorithms. In particular, these methods will be employed for the NCAR/DOE Community Climate System Model (CCSM). The proposed work is divided into three tasks: (1) identify canonical components of current and near future land-surface/terrestrial models and associated GIS layers and develop and interface to the CCSM flux coupler that allows simple addition and replacement of these components; (2) define equilibrium benchmarks (critical pools and fluxes of energy, latent heat, carbon, nitrogen, food web biomass distributions) and objective functionals and develop optimization methods to adjust parameters and initial conditions for biogeochemistry models to be consistent with current climate conditions simulations; and (3) develop optimization/data assimilation schemes to handle non-equilibrium conditions that are required for changing climate-terrestrial interactions over the past 200 years with changing atmosphere land-use change.

\section{Mission Relevance}

DOE has a major deliverable from its climate change research to "Deliver improved climate data and models for policy makers to determine safe levels of greenhouse gases for the Earth system" (www.er.doe.gov/ober/ober_top.html). DOE's Scientific Discovery through Advanced Computing (SciDAC) programs will support this endeavor to help meet their mandate to contribute to the CCSP, in particular their role in development of the Community Climate System Model (CCSM) in partneship with the National Science Foundation (NSF). The Climate Change Research Division contributes to this deliverable through Terrestrial Carbon Processes (TCP), Ocean Science (OS), and National Institute for Climatic Change Research (NICCR) to enhance research on coupled carbon cycle-climate research for DOE's program requirements that are more energy focused than the goals of the NSF's CCSM effort. Remote sensing data streams will eventually become an important benchmark that our enabling technology could use for appropriate initialization of models in climate and biogeochemistry forecasting. Our approach could be extended to combine satellite remote sensing with climate and earth system models.

\section{Results and Accomplishments}

The main techniques of the project can be summarized as developing a process to adjust the initial states of a model in such a way that outcome of model calculations is close, in some distance metric or cost functional, to observations. In our case, we are developing a method of 
adjusting the initial terrestrial carbon pools with different turnover times so that the ensuing model simulations are close to the observed seasonal shifts in atmospheric $\mathrm{CO}_{2}$ concentrations. These are coarse woody debris (CWDC, 1 to 18 subpools depending on model, litter (LITTERC, 3 to 6 subpools), live wood (WOODC), and soil carbon (SOILC, 2 to 4 subpools). This task is to define the cost functionals and assemble a useful set of observed states. The development of methods to make the adjustments is the work of task 3 .

The datasets that contain considerable information for these compartment pools are terrestrial biomass, soil C, and for leaf litter. Estimates of carbon storage in vegetation have, in some cases, been superseded by more recent studies at the national and regional level. Total carbon stores in soils an estimated global stock of organic carbon in the upper $100 \mathrm{~cm}$ of the soil to be between $1,462 \mathrm{GtC}$. For litter carbon, a new database was spatially mapped for use in this project.

A source to source tool, called Tangent linear and Adjoint Model Compiler (TAMC), was used to determine the gradient of the cost function. The process of generating the adjoint of our cost functional for the land-surface model proved to be difficult. TAMC was unable to generate the adjoint code for the model in its initial state due to source code restrictions and the size and complexity of the model. The code was changed in order to match TAMC strict requirements. To overcome the size restriction, we divided the code into several parts, obtained their adjoint independently, and then merged them back together. This process proved successful, although other problems arose. Specifically, intermediate variable dependency on the control variables was lost during this process, and hands-on, tedious, adjoint code verification had to be performed. These difficulties may affect our ability to apply this approach to all three models as outlined in the proposal.

The function calculation by the forward model and the gradient calculation by the adjoint model are input into a gradient-decent optimization algorithm that is used to perform control variable search. We tested several algorithms and decided on the conjugate-gradient search method. It is for unconstrained parameter condition, but we applied it to bounded conditions by assigning extremely large bounds in our application.

Using the uncoupled terrestrial model, we performed simulations for single points and for points in a relatively small region with a strong gradient in biomass and soil carbon pools. We used the usual spin-up procedure using periodic forcings to derive the quasi-periodic equilibrium state. We perturbed carbon pool turnover times and then used the data assimilation procedure to regain the previous initial conditions. In general, if the parameter perturbations were greater than a very small fraction of the initial values, the "true results" were only rarely recovered. There is not enough information in the aggregated pool estimates for a single time slice to constrain the optimization. Additional observational data must be brought to bear on this problem. A time series of information on pool sizes, or inferences on isotopic measurements, will be required to constrain estimates of turnover times of especially the older carbon pools.

The adjoint method, while potentially the most rigorous and efficient approach, proved to be too difficult for the class of models and the sparse benchmark datasets available. We were successful in applying this approach to other global change modeling problems; however, for the 
application on the other approach, ensemble Kahlman filter combined with a richer array of data constraints is required.

\section{2: Terascale Simulation Tools for Next-Generation Nuclear Energy Systems}

Kevin T. Clarno, Valmor F. de Almeida, Eduardo F. d'Azevedo, Ahmed Khamayseh, and Cassiano R. E. de Oliveira

\section{Project Description}

The primary goals of this LDRD project were to develop and demonstrate a state-of-the-art computational tool for high-fidelity heat generation (neutronics) simulation in nuclear reactors and formulate the computational science infrastructure for coupled physics simulation. The primary software development focused on the creation of a new high-fidelity radiation transport solver that is designed specifically for terascale computing architectures; the resulting software provides extensive and accurate detail of all relevant quantities (isotopic distributions, radiation fields, activation products, heat generation, etc.) on an unsurpassed differential level, yet with full system interaction, as needed for other multiphysics simulation codes. The generated information includes detailed data about heat sources needed for heat transfer, fluid flow, and structural mechanics. The developed computational science infrastructure enables a single mesh handling interface, solver coupling strategy, and data analysis and visualization mechanism for all associated physics that may eventually be coupled.

The three major objectives that this project addressed were as follows: (1) develop and assemble the computational infrastructure for efficiency and interoperability, (2) develop a high-fidelity radiation transport code for terascale parallel computing, and (3) demonstrate a radiation transport simulation.

\section{Mission Relevance}

The Global Nuclear Energy Partnership (GNEP) is a primary objective of DOE for the advancement of nuclear energy and closing the nuclear fuel cycle. The development of an applied high-fidelity neutronics solver and coupled physics reactor simulation is critical to the DOE Office of Nuclear Energy for the optimized design and safety analysis of advanced nuclear reactors proposed within GNEP and the Generation IV program. The DOE Office of Advanced Scientific Computing Research is also interested in the advancement of ultrascale computing simulation of nuclear energy systems for GNEP. Because of the university interactions, this project also addresses the DOE goal of training the next generation of leaders in nuclear engineering and computing simulation.

\section{Results and Accomplishments}

Significant accomplishments were made in each of the three objectives. (1) The infrastructure for a multiphysics simulation suite was developed. This infrastructure utilizes the common 
component architecture developed within the Scientific Discovery through Advanced Computing (SciDAC) program. A module (SCALECC) was adapted from a driver for the Standardized Computer Analysis for Licensing Evaluation (SCALE) nuclear data processing software to incorporate and process, in parallel, accurate nuclear data within a coupled-physics, highperformance computing environment. (2) A new parallel three-dimensional radiation transport solver (NEWTRNX) for simulation of nuclear systems was designed and developed specifically for high-performance computing architectures with novel parallel communication and iteration strategies, which also incorporate advanced computational mathematics toolsets. The NEWTRNX solver is based on the Extended Step Characteristics method and incorporates an asynchronous version of the Parallel Block-Jacobi parallelization strategy. The global solution is accelerated with a novel nonlinear multigrid algorithm developed specifically for this project. A module for geometry and mesh handling, the Geometry and Mesh Adaptivity Server (GMAS), which will coordinate all of meshing challenges in the multiphysics simulation, was developed. GMAS presently reads a mesh file, which can be generated by any of several mesh generation tools and is coupled with SCALECC and NEWTRNX. (3) Two demonstrations were developed to display the capability of the solver for massively parallel nuclear energy simulations of a finemesh nuclear fuel rod with tremendous geometrical structure and a simulation of the neutron distribution within the entire core of a full nuclear reactor vessel. The results were successfully completed.

\section{Publications}

Clarno, K. T. 2007. "Implementation of Generalized Coarse-Mesh Rebalance in NEWTRNX for Acceleration of Parallel Block-Jacobi Transport," Transactions of the American Nuclear Society 97.

Johnson, S. R. and K. T. Clarno. 2007. "Implementation of Transport Synthetic Acceleration in NEWTNRX," Transactions of the American Nuclear Society 97.

Hamilton, S. P., C. R. E. de Oliveira, and K. T. Clarno. 2007. "Time-Dependent Radiation Transport Using the Slice-Balance Method," Transactions of the American Nuclear Society 97.

\section{5: Multiscale Modeling: Application to Hydrogen and Helium in Steels}

D. M. Nicholson, P. K. Nukala, Yu. N. Osetskiy, R. E. Stoller, S. Namilae, and C. Y. Gao

\section{Project Description}

We propose to build a multiscale framework for the modeling of dislocations in iron alloys containing small impurity atoms, hydrogen, and/or helium. This framework will immediately have a much broader range of application, provide better understanding of the role of interstitial solutes such as carbon, and promote growth in other areas of materials modeling. However, we take the very pragmatic view in that the urgency of the scientific and energy relevance of this research requires us to focus on realistic models rather than toy systems and to go directly after 
the science issues. Those issues are the mechanical properties of steels to be employed in advanced nuclear (fission and fusion) energy systems, in the pipelines and tanks required for a hydrogen economy, and in the target at the Spallation Neutron Source. Mechanical failure in any of these applications could render them unviable. The goal of this proposal is to ultimately link nanoscale structural evolution and mechanical properties in a predictive way.

\section{Mission Relevance}

This work is relevant to structural materials research within the U.S. Department of Energy in the areas of transportation, energy production, and energy delivery. We will advance the ability to model mechanical properties of metals, thereby benefiting a large cross section of industries. In particular, the mechanical properties of iron alloys in the presence of hydrogen and helium are important to the delivery and storage of hydrogen for the "hydrogen economy" and for the next generation of nuclear reactors.

\section{Results and Accomplishments}

Advances have been made in software, computed results, and formalism. We have incorporated molecular dynamics into the parallel finite-element code Nike3D. This implementation uses a finite-element (FE) mesh refined to the atomic scale in a handshaking region with a corresponding Hamiltonian (introduced by Vashishta) that determines dynamics. The design is such that other molecular dynamics (MD) codes in use at ORNL and elsewhere can easily utilize FE boundary conditions in our FE-MD-Nike3D code as an alternative to more restrictive periodic boundary conditions. A new multiscale formalism based on correlation functions has been introduced; many of the concepts were published. The formalism is further developed in a manuscript still in preparation.

The Greens function boundary method was implemented for quasi-static, that is, Newtonian, dynamics followed by relaxation via the Greens function. Results were obtained for an edge dislocation interacting with a 2-nm void in bcc iron. Favorable comparisons were made to calculations using the Periodic Array of Dislocations (PAD) procedure introduced by Baskes and Daw. The Green function boundary condition (GFBC) removes unphysical forces between dislocations and their periodic images that occur in the PAD method. PAD calculations are made with very large numbers of atoms in the cell to reduce the effect of image forces resulting from periodic boundary conditions. GFBC calculations give results similar to PAD simulations using much larger cells. Both give the same Peierls stress of $24 \mathrm{MPa}$ and similar critical strain [0.033\% (GFBC) and $0.035 \%$ (PAD)].

Several hundred density functional results of atomic forces and energetics of defects in iron with or without hydrogen were used to quantify the energy and magnetization landscape of the FeH. Embedded atom force functions for the FeH system were developed based on the first-principles results. Calculations of the binding energy of hydrogen were calculated as a function of distance from an edge dislocation in iron.

We have proposed a global Hamiltonian that describes an atomistic region representing a single event such as a moving dislocation that is embedded in a thermally averaged coarse-grained region. The formalism is based in part on classical density functional theory but at a higher order 
than was typically used. We have formulated the functional to be applicable to a MD region embedded in a FE region. The central quantity in this formalism is the anisotropic pair distribution function (PDF), PDF's are usually treated as isotropic as in fluids. We have derived an expression for the entropy as a functional of the PDF that is valid for both the fluid and crystalline phases.

Our work on absorbing boundary conditions started with a theory for outgoing waves in a system described by partial differential equations. A number of analytic and simulated results have been obtained for 1-d systems with nearest-neighbor harmonic interactions. Our initial code was for a transverse elastic wave in one dimension; however, our real interest is in the interface of MD with $\mathrm{FE}$ in $3 \mathrm{~d}$. We have put absorbing boundary conditions into out FEMD code and demonstrated the effectiveness of the method.

\section{Publications}

Namilae, S., et al. 2007. "Absorbing Boundary Conditions for Molecular Dynamics and Multiscale Modeling." Phys. Rev. B. 76, 144111.

\section{6: Exploring Performance Tools for Petascale Systems with Lightweight Compute Node Kernels}

Philip C. Roth, Richard F. Barrett, and Jeffrey S. Vetter

\section{Project Description}

A fundamental part of the DOE Office of Science's leadership-class computing strategy is the use of systems like the Cray XT with a large number of simple compute nodes, each running a lightweight operating system kernel (LWK). Such systems are attractive for large-scale scientific computing because their lightweight kernels enable application scalability and performance predictability. However, these systems present a challenging target for performance, correctness, and system administration tools. Large numbers of application processes stress tool scalability, and lightweight kernels lack higher-level operating system services used by tools to monitor and control application processes. We propose to investigate petascale performance tools in the context of LWK systems like the Cray XT system deployed at ORNL. First, we will examine the feasibility of dynamic software-based instrumentation techniques on such systems. This type of instrumentation can adapt its behavior to the performance phenomena of the application and system, enabling a user to gain performance insight as the application executes. Second, we will explore the use of hierarchical communication and data aggregation techniques for tools on LWK systems. Our earlier work with hierarchical overlay network infrastructure explored such techniques on systems running full operating system kernels, but their use on LWK systems remains unexplored. Finally, we will investigate the automation of performance-analysis techniques like multivariate statistical analysis on systems with lightweight compute node kernels. Automated techniques enable users to distill insight from their application's behavior without requiring them to become performance-analysis experts. 


\section{Mission Relevance}

This research addresses ORNL's long-term objectives in the Terascale Computing and Simulation Science initiative by investigating techniques for analyzing the performance of scientific codes at scale. The work responds to the programmatic opportunity for establishing ORNL as a world leader in capability computing and in scalable tools. Because the techniques we investigate can also be used on systems with traditional compute node kernels and in correctness (e.g., debugging) and system administration tools, our results generalize to a wider class of tools and systems than our initial target platform to support ORNL's leadership computing strategy. Our results also benefit other agencies with significant interest in petascale computing, including the Department of Defense and the National Security Agency. Funding to continue some of this project's research directions is expected via DOE's three-year Forum to Address Scalable Technology for Runtime and Operating Systems (FAST-OS) program.

\section{Results and Accomplishments}

During FY 2007, the project's second year, we made significant progress in three research directions: petascale performance tool infrastructure, scalable performance data visualization techniques, and performance data collection infrastructure. In the first research area, we investigated the impact of performance and debugging tools using hierarchical overlay network software for communication and data reduction on systems with three-dimensional mesh interconnection networks such as the Cray XT. Although using such overlay networks can improve tool scalability, they consume system resources that are then unavailable to scientific applications. To provide insight into how many resources to devote to tools, we explored the relationship between the amount of tool resources and the amount of scientific computing work achieved in a fixed allocation of computing time. In the second research area, we developed an interactive, three-dimensional performance data visualization tool. The tool displays a representation of Cray XT compute nodes and the network that connects them. In support of our investigation of the impact of tools using hierarchical overlay network software, we used this visualization tool to display the relative locations of the compute nodes allocated by the Cray XT resource manager and the system's service and input/output (I/O) nodes. In the third research area, we developed a prototype event-tracing infrastructure for the Cray XT that enables collection of performance data describing selected application events without application source code modifications. We used this infrastructure to capture the I/O behavior of several applications of interest to the DOE Office of Science.

\section{Publications}

Barrett, R. F., S. W. Poole, and S. R. Alam. 2008. Expressing POP with a Global View Using Chapel: Toward a More Productive Ocean Model. ORNL/TM-2007/122, Oak Ridge National Laboratory.

Barrett, R. F., P. C. Roth, and S. W. Poole. 2008. Finite Difference Stencils Implemented Using Chapel. ORNL/TM-2007/119, Oak Ridge National Laboratory.

Roth, P. C. 2006. "On-line Automated Performance Diagnosis on Thousands of Processes." 2006 ACM SIGPLAN Symposium on Principles and Practice of Parallel Programming (PPoPP'06), May 29-31, 2006, Manhattan, NY, USA. 
Roth, P. C. 2007. "Characterizing the I/O Behavior of Scientific Applications on the Cray XT." Petascale Data Storage Workshop, Co-located with SC07, November 11, 2007, Reno, NV, USA.

\section{0: Large-Scale Exploration of Protein Models for System Biology Applications}

Andrey Gorin, Andrew Bordner, Yuan Tian, Xiuping Tao, Thomas Schulthess, and Yaohang Li

\section{Project Description}

We propose to build a high performance computing (HPC) implementation into ROSETTA-the leading ab initio protein-folding program. The proposed capability immediately will be in high demand from the system biology community; it will also foster new research and enhance ORNL's goals in long-term computer science and computational biology programs. The proposed work is divided into three tasks: (1) implement ROSETTA on the HPC architectures;

(2) assemble libraries for the smooth transfer of the existing and future biomodeling applications to HPC platforms; and (3) couple the platform with the previously developed algorithms for protein functional characterization and conduct interactive runs on the whole genome of $R$. palustris.

\section{Mission Relevance}

This research will provide significant impact to DOE's mission in system biology through the extensive structure modeling of the proteins of $R$. palustris and other bacteria, which is important to energy production research. The research focuses on modeling hypothetical proteins using terascale computer power. The determined structure will provide multiple opportunities for further analysis and may immediately point to the range of possible functional roles for this sequence. The most relevant DOE programs are the Genomics:GTL program and planned DOE Biofuel Centers.

\section{Results and Accomplishments}

During FY 2006, we implemented the Parallel Monte-Carlo Rosetta platform and conducted several types of folding experiments on $\sim 500$ functionally important protein domains. It was shown that the Parallel ROSETTA has a unique capability to cross high potential energy barriers, indicating the possibility of reaching full Monte Carlo convergence for the shortest protein domains.

This technological breakthrough led to a significant increase in the model quality across the whole investigated benchmark. In the subset of the shortest domains, the achieved improvements have crossed a crucial threshold, as high-quality structures were obtained for $75-88 \%$ of all tested sequences. With this success rate, it is feasible to apply this technology to system biology problems (e.g., an annotation of the hypothetical proteins in the targeted genomes). 
During FY 2007, we implemented novel Common Kernel algorithm for identification of the near native structures. The ROSETTA program generates results as a very large ensemble of structural models, with a few of them often closely resembling the native structure. The selection of the model closest to the native structure is a difficult but crucially important problem, as only successful folding attempts can be interpreted for clues about biological function.

The Common Kernel algorithm has two important properties: (1) The group with the largest Common Kernel in $90 \%$ of the cases includes the best near-native structure present in the ensemble and (2) The size of the Common Kernel (calibrated by the protein chain length) provides an accurate estimate of how close the structure is to the native one.

The developed capability provides a new way for a systematic exploration of the hypothetical proteins in the targeted bacterial organisms.

\section{6: Exploring Reconfigurable Computing Programming Models to Accelerate High-Performance Computing (HPC) Applications}

O. O. Storaasli

\section{Project Description}

High-Performance Computing (HPC) vendors see field-programmable gate array (FPGA) accelerators as a next major performance advance. Silicon Graphics, Inc. (SGI) predicts FPGAs will "accelerate mission-critical applications by over 100 times." Cray selected DRC FPGA coprocessors for future XT supercomputers because they offer "the greatest opportunity for application acceleration," enabling new scientific breakthroughs to solve previously intractable problems. New algorithms are needed to effectively harness FPGAs for large science codes, a challenge requiring bottom-up FPGA expertise and a top-down HPC application developer/user perspective. This research proposes a computational framework to speed DOE and ORNL science codes by placing their compute-intensive kernels (floating point, fast Fourier transforms, matrix and linear algebra, etc.) on FPGAs via appropriate languages/ tools, demonstrating computation speedup via selected "pathfinder" applications charting a way others may follow. This research leverages close collaboration with Xilinx's HPC group, Cray, SGI, Mitrion, University of Tennessee (UT), the National Science Foundation (NSF) Center for HighPerformance Reconfigurable Computing (CHREC) at the University of Florida, OpenFPGA.org and others.

\section{Mission Relevance}

This research supports the DOE's Office of Science Ultrascale Computing Initiative and the President's Information Technology Advisory Committee (May 2005) as being "critical to scientific leadership, economic competitiveness, and national security." This research plus a recent $\$ 700 \mathrm{~K}$ follow-on DOE contract (awarded to Alpha Star and ORNL) and two UT Science Alliance grants directly benefit the Defense Advanced Research Projects Agency (DARPA) and National Science Foundation via ORNL's Center for High-Performance Reconfigurable 
Computing membership, as well as the U.S. Air Force, Army, Department of Homeland Security, National Security Agency, and National Aeronautics and Space Administration. The research results demonstrate how key DOE codes (bioinformatics, climate/weather, molecular dynamics and matrix equation solution) can achieve major performance gains (up to $100 \times$ speedup) by harnessing field programmable gate array (FPGAs), leading the way for other codes to follow. FPGA acceleration is attractive for future ORNL supercomputer applications to exceed PetaFlop performance, enabling major scientific breakthroughs.

\section{Results and Accomplishments}

This research was expanded/accelerated via no-cost additions of Xilinx's CHiMPS HPC team, a UT professor and full-time Ph.D. student via two Science Alliance grants, and contributions of FPGA systems, software and development tools (Cray, SGI, Mitrion, Viva, DSPlogic, etc.). Bioinformatics, molecular dynamics, matrix solution, and weather/meteorology codes were successfully ported to FPGAs with up to $100 \times$ computation speedups achieved/published. FPGA speedups of $50 \times$ and $100 \times$ (over a $2.2 \mathrm{GHz}$ Opteron) were achieved for human-genome DNA matching on Cray XD1s with Virtex-II and Virtex-4 FPGAs, respectively. These speedups scaled (increased) with both the number of FPGAs used and the database query size. The molecular dynamics code, ported to both Xilinx and Altera FPGA systems, achieved 7× overall speedup. A new mixed-precision matrix solver achieved $10 \times$ overall speedup $(36 \times$ for the matrix factor kernel) on a FPGA-enabled Cray XD1. Research results from this project were presented and well received at 23 meetings and conferences, including 11 invited presentations and 5 international conferences. The DNA matching results were highlighted in a tutorial entitled "The Promise of FPGA-Acceleration Processors to Bioinformatics Research" at the Ohio Collaborative Conference on Bioinformatics, July 9-11, 2007. Results from this project were reviewed favorably by and included on the FY 2008-09 OpenFPGA.org web site. A recent "spinoff" of this research is the award of a \$700K DOE FY 2008-09 small business contract (to our Alpha Star - ORNL team) to apply our parallel/FPGA-based methods to accelerate progressive failure analysis of structures.

\section{Publications}

Storaasli, O. 2006 "Future High-Performance Computing," International Conference on Computational Science and Education, Rochester, NY, Aug 7-10.

Storaasli, O. O. and D. Strenski, 2007. "Accelerating Genome Sequencing 100X with FPGAs." Reconfigurable Systems Summer Institute (RSSI) 2007, National Center for Supercomputer Applications (NCSA), July 17-20, 2007, Urbana, IL, USA.

Storaasli, O. O. and D. Strenski. 2007. "Exploring Accelerating Science Applications with FPGAs." Reconfigurable Systems Summer Institute (RSSI) 2007, National Center for Supercomputer Applications (NCSA), July 17-20, 2007, Urbana, IL, USA.

Storaasli, O. O., et al. 2007. "Performance Evaluation of FPGA-Based Biological Applications." Cray Users Group 2007, May 7-10, 2007, Seattle, WA, USA. 
Storaasli, O., J. Vetter, M. Smith, P. Roth, and S. Alam. 2006. "Improving Programmer Productivity for Multi-Paradigm Computing (whitepaper)," DARPA FPGA HPC Workshop, Arlington, Virginia, April 27-28.

Storaasli, O., M. Smith, and S. Alam. 2006. "Experiences Harnessing Cray XD1 FPGAs and Comparisons to Other FPGA High Performance Computing (HPC) Systems," Cray User Group 2006 Proceedings, Lugano, Switzerland, May 8-11.

Sun, J., G. D. Peterson, and O. O. Storaasli. 2007. "Mapping Sparse Matrix-Vector Multiplication on FPGAs." Reconfigurable Systems Summer Institute (RSSI) 2007, National Center for Supercomputer Applications (NCSA), July 17-20, 2007, Urbana, IL, USA.

Sun, J., G. Peterson, and O. Storaasli. 2007 "Sparse Matrix-Vector Multiplication Design on FPGAs," IEEE FCCM07, Napa Valley, CA, April 23-25.

Vetter, J., M. Smith, P. Roth, O. Storaasli, and S. Alam. 2005. "Programming Tools, Reconfigurable Computing," Reconfigurable Computing BOF, 8th Military and Aerospace Programmable Logic Devices (MAPLD) International Conference, Washington, D.C., September 7-9.

\section{8: Predictive Simulation and Virtual Design of High-Speed, High- Density Molecular and Nanoscale Sensors and Devices}

Vincent Meunier, Edoardo Apra, Jerzy Bernholc, Robert Harrison, William Shelton, and Bobby Sumpter

\section{Project Description}

Theoretical methods have recently evolved to a point where the properties of materials can be successfully predicted based solely on their atomic structure and without any experimental input. Furthermore, simulation can provide a "theoretical microscope," by identifying the origins of the various properties of a given structure and by uncovering principles that can be used to systematically enhance the desired characteristics or to suppress the unwanted ones.

Unfortunately, current first-principle methods and software can handle only a few hundred atoms. Developing scalable methods capable of fully utilizing petascale systems would enable predictive simulations of entire device structures from first principles, thereby revolutionizing the design of molecular and nanoscale sensors and electronic devices. Our expertise in quantum methods, applied mathematics, and large-scale computing uniquely positions us to establish ORNL as a leader in the emerging field of predictive design of nanodevices.

\section{Mission Relevance}

Developing scalable methods that can fully utilize petascale systems would enable predictive simulations of entire device structures from first principles, thereby revolutionizing the design of molecular and nanoscale sensors and electronic devices. This virtual design would impact many areas critical to DOE's needs, such as the development of specific biosensors with a nearly single-molecule detection limit, and of ultradense, ultrafast, molecular-sized electronic components, with very small power requirements and persistent, reprogrammable memories. The 
proposed work will also benefit other agencies, including the National Science Foundation (NSF), DoD, and National Institutes of Health (NIH). NSF is expecting multiple program calls in the development of petascale software and its applications in several of its directorates. We also expect additional calls for proposals in areas of strategic DoD interests. NIH has already solicited proposals for its Centers in Nanomedicine.

\section{Results and Accomplishments}

The code used to compute the non-equilibrium transport properties was rewritten to allow for computing the Green functions using an iterative method (rather than a direct inversion). We also reorganized the algebra of the problem to facilitate multilevel parallelization. The three levels of parallelization we completed are parallelization over energy integration, over the matrix operation, and over bias in I-V calculations. Functional materials for device or energy application require a large number of atoms to be included in the active region of the system. Treating such systems is a true computational challenge for a first-principles approach since more that a few thousand atoms are usually needed to achieve good accuracy. To address the electronic structure, we have implemented the Pseudopotential Algorithm for Real-Space Electronic Calculations (PARSEC) on the Cray XT4 at the NCCS. A considerable amount of optimization had to be performed. The optimization included a new parallel distribution of the real-space grid that enabled better memory management and parallel performance. Without these computational enhancements, the simulations become untenable because of poor memory management and scaling with system size. We also worked on porting of NWChem software on Cray XT4. This activity is coordinated with the developers of the Global Arrays library (GA) group from PNNL, because NWChem makes extensive use of GA for the interprocessor communication. The current GA port is not optimal because it is affected by both performance and stability issues. In order to alleviate these problems, we adopted a replicated-data style parallelization for the DFT code that limits the maximum size of problems we can deal with because the memory available on each processor becomes a bottleneck. In addition to technical achievements, we have also made a large number of scientific achievements with seven papers already published or submitted.

\section{Publications}

Drummond, M. L., V. Meunier, B.G. Sumpter. 2007. "Structure and Stability of Small Boron and Boron Oxided Clusters," J. Phys. Chem. A 111 (28), 6539-6551.

Souza Filho, G., et al. 2007. "Selective Tuning of the Electronic Properties of Coaxial Nanocables through Exohedral Doping," Nano Letters 7(8), 2383-2388.

Meunier, V., S. V. Kalinin, B. G. Sumpter. 2007. "Nonvolatile Memory Elements Based on the Intercalation of Organic Molecules Inside Carbon Nanotubes," Phys. Rev. Lett. 98, 056401.

Meunier, V., B.G. Sumpter. 2007. "Tuning the Conductance of Carbon Nanotubes with Encapsulated Molecules," Nanotechnology 18, 424032. 


\section{9: Storage Virtualization: An Integrated Approach to Machine- Room Storage Management}

Sudharshan S. Vazhkudai, Xiaosong Ma, John W. Cobb, and Gregory G. Pike

\section{Project Description}

Procurement and optimized utilization of petascale supercomputers and centers is a renewed national priority. Sustained performance and availability of such large centers is a key technical challenge that will significantly impact their usability. As recent research shows, storage system faults, data unavailability, and I/O bandwidth bottlenecks can cause even today's supercomputers to fail. These problems significantly impact common supercomputing I/O operations such as data staging, offloading, checkpointing, and prefetching, leading to suboptimal high-performance computing (HPC) center performance, increased job turnaround time, frequent resubmissions and poor use of precious center resources, as well as users' allocated time. Solving these issues is highly critical to scaling to petascale systems. Modern HPC centers and users' job workflow offer numerous opportunities for significant improvements in the storage hierarchy that have gone unnoticed. This research takes a fresh look at the HPC storage crisis with an eye toward virtualizing the entire center as a system. In this setting, we will perform the following: (1) global coordination and scheduling of data and computational activities, (2) the construction of novel storage abstractions using untapped storage resources available in the machine room, and (3) ensure conjoined use with each other and with traditional storage elements.

\section{Mission Relevance}

The DOE is building a leadership-class computing facility (LCF) at ORNL, which is expected to scale to a petaflop machine in the coming years. Successful delivery of petaflop capability is dependent on robust computing, storage, and networking environments. Success in this project will enable the delivery of robust storage solutions for the LCF. This can significantly improve the usability, performance, and uptime of the LCF, all of which are of high importance to DOE. In addition to DOE, the National Science Foundation and Defense Advanced Research Projects Agency are soliciting proposals for large-scale HPC acquisitions. Machine uptime and productivity (percentage user jobs successfully completed without resubmissions) are key metrics for measuring the success of these high-end petascale machines. I/O and storage problems being addressed by this project are of significant importance to the HPC solicitations from these agencies. Thus, solutions from this research will be of much value to these agencies as well.

\section{Results and Accomplishments}

We have designed a framework that performs global coordination of data and computes activities, enables the plugging in of traditional parallel file system and construction of new storage abstractions, and facilitates seamless communication between the disparate storage elements. Using this framework, we have achieved the following two deliverables:

We have designed a novel job execution environment, in which data staging is scheduled in coordination with jobs. Our approach involves the explicit definition of data staging and 
offloading in a job script, followed by its decomposition and dependency setup. By scheduling data alongside computing, we have ensured that the data will have arrived when the job is ready to be scheduled. Likewise, upon job completion, the offload job is scheduled to transfer the data to its destination, thereby avoiding being purged while awaiting manual intervention.

We have built transparent data reconstruction to protect the staged data against storage system failures. This builds a seamless communication path from the parallel file system to archives or network file system, thereby virtualizing I/O access to where the original staged data might be available.

Due to our coordinated scheduling and reconstruction of staged data, average job wait times were dramatically reduced. In fact, we can achieve a very similar average job wait time as when no failure occurs. Compared to extant solutions, our approach involves no manual data movement, optimal scratch space use, and no charge to user-allocated time. It also reduces the average waiting time of jobs and increases overall center throughput and service.

\section{Publications}

Al Kiswany, S. I., M. Ripeanu, and S. S.Vazhkudai. 2008. A Checkpoint Storage System for Desktop Grid Computing. ORNL/TM-2007/102, Oak Ridge National.

Ma, X., Z. N. Zhang, and S. S. Vazhkudai. 2008. Improving Data Availability for Better Access Performance: A Study on Caching Scientific Data on Distributed Workstations. ORNL/TM-2007/103, Oak Ridge National Laboratory.

Vazhkudai, S. S. and X. Ma. 2007. "Recovering Transient Data: Automated On-demand Data Reconstruction and Offloading for Supercomputers." ACM SIGOPS OSR. 41, 14-18.

Vazhkudai, S. S., et al. 2008. A Checkpoint-Optimized Storage System for HPC Applications. ORNL/TM-2007/104, Oak Ridge National Laboratory.

\section{0: Virtualized Systems Environment for Petascale Computing and Beyond}

Stephen L. Scott, Hong Ong, Christian Engelmann, Geoffroy Vallee, Ricky Kendall, Ron Brightwell, and Barney Maccabe

\section{Project Description}

DOE is deploying petaflop computing. In order for systems this size to run "out-of-the-box," several challenges in petascale system software and application runtime environments have to be addressed to ensure day-one operation. Efficiently exploiting tens to hundreds of thousands of processor cores using tens to hundreds of thousands of interdependent computational tasks requires appropriate scalability, manageability, and ease of use at the system software and application runtime environment level. Furthermore, the expected system upgrade interval demands an incremental strategy for scientific application development and deployment that avoids excessive porting. This project addresses these issues at the system software level through the development of a virtual system environment (VSE). In addition to providing a scalable and 
reliable "sandbox" environment for scientific application development on desktops and clusters, the VSE will offer an identical production environment for scientific application deployment on terascale and petascale high end computing (HEC) systems. The proposed VSE concept enables "plug-and-play" supercomputing through desktop-to-cluster-to-petaflop computer system-level virtualization based on recent advances in hypervisor virtualization technologies. The goal of the proposed effort is to advance the race for scientific discovery through computation by enabling day-one operation capability of newly installed systems and by improving productivity of scientific application development and deployment.

\section{Mission Relevance}

This work is relevant to the DOE Office of Science program for operating/runtime systems for extreme-scale scientific computing by addressing the Ultrascale Computing initiative in the area of computer science and mathematics. More specifically, it deals with the issues of (1) developing scalable, fault-tolerant system software and runtime technologies to enable the efficient utilization of petaflop computers and (2) developing tools that increase the productivity of the application development environment for petascale systems. It also benefits the National Science Foundation's plan to develop computer and information science and engineering program for software and tools for high-end computing and will benefit the Defense Advanced Research Projects Agency's Information Processing Technology Office.

\section{Results and Accomplishments}

This first year's effort has resulted in a number of prototypes, including tools for system-level virtualization and a hypervisor solution for HEC: (1) OSCAR-V for the management of VSEs. Designed and implemented VSE management tools, including V2M, and combined it into the OSCAR-V prototype release. We initiated a study of OSCAR-V and Dynamic Virtual Cluster integration to interface VSE management tools with job and resource management tools to provide on-demand VSE deployment. (2) Resiliency framework prototype for pro-active fault tolerance. This prototype utilizes virtual machine (VM) migration technology that may preemptively move computation away from unhealthy compute nodes by moving the entire VM instance to another compute node. This prototype is based on Xen live migration and VM pause/unpause. This prototype is being extended to support the lightweight hypervisor effort and integration into OSCAR-V. (3) Lightweight variant of the Xen hypervisor. We developed this customized hypervisor, specifically targeting HEC. This implementation was done in a way that enabled existing monitoring and profiling tools for the Xen hypervisor to be reused for the lightweight hypervisor. (4) Porting of Catamount to the XenoLinux interface. This allows Catamount to run on top of Xen. (5) A new hypervisor specifically targeting HEC. Current implementation provides a simple hypervisor for Intel processors that support the VT-X extensions. (6) XML specification. This specification allows users to specify sets of software packages and to create images for VMs based on this package set. Respective package set management tools have been added to OSCAR and OSCAR-V. Initial support for automated VM deployment also includes an optional "mapping" of VMs to host operating systems. 


\title{
Publications
}

Vallée, G. R., T. Naughton, and S. L. Scott. 2007. "System Management Software for Virtual Environments," Proceedings of ACM Conference on Computing Frontiers 2007, Ischia, Italy, May 7-9.

Engelmann, C., S. L. Scott, H. Ong, G. Vallée, and T. Naughton. 2007. "Configurable Virtualized System Environments for High Performance Computing," Proceedings of the First Workshop on System-level Virtualization for High Performance Computing (HPCVirt 2007), Lisbon, Portugal, March 20.

Vallée, G. R., and S. L. Scott. 2006. "Xen-OSCAR for Cluster Virtualization,” Proceedings of the Workshop on Xen in High-Performance Cluster and Grid Computing Environments (XHPC'06), Sorrento, Italy, December 4-6.

Xin, J. 2007. Exploring the Lightest Virtual Machine Monitory Based on VT-x, Northwestern University, internal report.

Naughton, I., T. J., G. R. Vallée, and S. L. Scott. 2007. "Dynamic Adaptation using Xen.” First Workshop on System-level Virtualization for High Performance Computing (HPCVirt 2007), March 20, Lisbon, Portugal.

Vallée, G. R., et al. 2006. "Checkpoint/Restart of Virtual Machines Based on Xen." High Availability and Performance Computing Workshop 2006, October 17, 2006, Santa Fe, NM, USA.

\section{1: Petascale Computing in Nanoscience on 100,000+ Cores}

\author{
T. C. Schulthess, M. Eisenbach, D. Nicholson, X. Tao, C. Zhou, and J. Levesque
}

\section{Project Description}

The overarching goal of this project is to implement a general methodology for computing thermodynamic quantities in nanoscale systems that can make effective use of petascale computing systems with $10^{5}$ or more cores. Thermodynamics in nanoscale systems present new challenges that cannot be met with conventional techniques. Atomic-scale interactions change drastically even over short distances; indeed the mechanisms governing stability can differ between surface and interior of a nanostructure and are often dominated by entropic effects. In this milieu, the physics can be unraveled only at the first-principles level and combined with statistical methods to include entropy. Our approach combines a novel stochastic sampling scheme, the generalized Wang-Landau (gWL) method, to compute the density of states (and hence the entropy and free energy) of a nanoscale system with accelerated density functional theory (DFT) methods that have previously been scaled to thousands of processors. Unlike metropolis Monte Carlo, the gWL is not embarrassingly parallel, but we nevertheless anticipate that the combined $\mathrm{gWL} / \mathrm{DFT}$ codes will scale to $10^{5}$ or more processors.

During the first year, we studied statistical effect in realistic models of magnetic nanoparticles and demonstrated that the temperature-dependent free energy barrier for magnetic switching can indeed be computed with the gWL method. We found nontrivial nanoscale effects that will have to be investigated with ab initio techniques. We have implemented a gWL/DFT hybrid code 
where the DFT part is based on the LSMS code. We find near optimal strong scaling to the full size of Jaguar (20,000 cores) with efficiency in the $70-80 \%$ range.

\section{Mission Relevance}

The application codes, along with the methods and experience we develop in this project, will be part of the computational nanoscience endstation at ORNL. With this, the CNMS and NCCS user programs will gain a unique aspect and a competitive advantage over comparable facilities and efforts in the nation. This will allow us to enhance the programs in Basic Energy Sciences user facilities and ensure the scientific success of the next-generations NCCS machines through plugand-play petascale computing applications. In particular, this project will allow us to take on new staff scientists that will be available for programs in support CNMS and NCCS.

\section{Results and Accomplishments}

We met all first year deliverables and scientific/technical accomplishments can be summarized as follows: (1) We have developed a hybrid parallel programming model for the gWL method that will naturally scale to $\sim 10^{5}$ cores when combined with DFT codes. (2) We have implemented a prototype implementation of the gWL/DFT code with LSMS at its core and have demonstrated strong scaling to up to 20,000 cores on Jaguar. (3) We demonstrate that the $\mathrm{gWL} / \mathrm{DFT}$ codes will have similar performance as the underlying DFT code - in the case of gWL/LSMS, the sustained performance we reached is $70-80 \%$ of peak. (4) We showed that fast and nonetheless accurate computations of the magnetic energy of magnetic nanoparticles are possible with the LSMS code, and (5) By studying simpler models of magnetic nanoparticles we demonstrated that free energy barriers for switching can be effectively computed with the gWL method and that nontrivial nanoscale effects are present.

\section{2: Modeling Cellular Mechanisms for Efficient Bioethanol Production through Petascale Comparative Analysis of Biological Networks}

Andrey Gorin, Nabeela Ahmad, Andrew Bordner, Jessie Gu, Guruprasad Kora, Chongle Pan, Byung-Hoon Park, and Nagiza Samatova

\section{Project Description}

This project aims to characterize biochemical and regulatory machineries of ethanol-producing biological systems by scaling algorithms and advancing underlying theory in the graph analysis area. Graph theory naturally forms the mathematical language to formulate and solve problems on biological networks of genes, interacting proteins, and co-expressed gene products. Biological networks tend to be extremely large (up to millions of nodes) and complex (connectivity is nonuniform). This presents a significant computational challenge and motivates development of parallel algorithms, efficiently scalable to thousands of processors on LCF-like architecturesthe ultimate objective of this project. 
Our approach to the Library of Parallel Graph Algorithms centers on very efficient and highly parallelizable implementations for a few core algorithms (e.g., maximum click enumeration, vertex cover) used as building blocks for more complex algorithms with dozens of applications to bionetwork analysis and reconstruction. In the first year of this project, we demonstrated significant acceleration (up to 1000 times) and near perfect scalability (only $~ 30 \%$ loss of peak capacity on hundreds of CPUs) for several core algorithms. At this early stage, the acquired graph analysis capabilities were crucial for the success of the three different biological applications related to analysis of interaction and regulatory networks in microbial organisms and yeast.

\section{Mission Relevance}

DOE aims to replace $75 \%$ of U.S. oil imports with renewable alternatives by 2025 . The primary approach seeks to produce ethanol from plant biomass using microbial and fungal systems. This process involves degradation of lignocellulose to sugars; however, their fermentation to ethanol via biochemical pathways are poorly understood. We propose to investigate mechanisms to allow these pathways to function efficiently and to regulate them through computational modeling that will require petascale data analytics. The Office of Biological and Environmental Research and Office of Advanced Science Computing Research will benefit from advances proposed in this project.

In addition to benefiting DOE, comparative network analysis tools such as those proposed here would have an impact akin to Blast-like comparative genomics tools in the human genome project. As such, the National Institutes of Health and the National Science Foundation programs dealing with systems-level understanding of complex biological systems, especially comparative evolutionary analysis of metabolic, regulatory, and signaling pathways will benefit from these innovations.

\section{Results and Accomplishments}

During FY 2007 we made significant progress toward developing a generic library of graph algorithms that are scalable to petascale. These include the following: (1) Faster solutions for the maximum common subgraph (MCS) problem. We have transformed an essential part of the MCS search process to the task of enumerating maximal independent sets in a much smaller graph. Implementation of the resulting algorithm produces speedups ranging from 2 to 1,000 on a single processor. The critical part of the search is amendable to parallelization. (2) The buffered work-pool (BWP) approach to search-tree-based optimization algorithms. We proposed the BWP as novel load-balancing strategy for a wide variety of platforms. The BWP-based MCS algorithm was compared with a sequential version on graphs from DIMACS Benchmarks. (3) Reaching nearly perfect scalability for clique enumeration. We implemented a new approach to parallelization of a backtracking maximal clique enumeration. Parallel implementation of the algorithm shows almost perfect scalability: at 244 processors, the performance is only $30 \%$ behind ideal parallelization. Using the developed graph analysis capabilities, we obtained important results in several relevant biological systems, such as (1) Analysis of protein coexpression in Rhodopseudonomas palustris (microbe capable of degrading aromatic compounds and releasing $\mathrm{H}_{2}$ ). We proposed a new conceptual mechanism balancing activity of its crosscutting biochemical pathways. (4) Development of a framework for inferring protein functional 
modules from mass-spectrometry pull-down data. The identified associations provide clues to biological processes characterizing the growth condition of the yeast cell, indicating mechanisms activating stress conditions in the yeast cells, including stress conditions of ethanol production.

\title{
00014: An Evolutionary Framework for Porting Applications to Petascale Platforms
}

\author{
Kalyan S. Perumalla, Nikhil Bhatia, Phani Teja Kuruganti, Jeffrey Vetter, and Vladimir \\ Protopopescu
}

\section{Project Description}

This project investigates a novel approach to petascale computing that addresses the severe synchronization overheads that inherently emerge with a large number of processors. Inherent tight coupling among processors is relaxed by incrementally and semitransparently enabling speculative computing using novel synchronization mechanisms such as reverse computation. The envisioned framework attempts to move beyond traditional checkpointing-based approaches to newer directions in reversible computing to free applications from tight global coupling. It enables easily porting existing MPI-based applications and moving them in a backwardcompatible, evolutionary fashion to petascale computing platforms without having to restructure existing codes. The overall effort offers significant benefits along multiple directions, including new scientific techniques such as reverse computation-based relaxed parallel execution, new petascale extensions to existing message-passing standards, and least-effort paradigms to porting existing applications for potentially significant productivity and efficiency improvements.

\section{Mission Relevance}

The project will benefit supercomputing applications, such as large-scale scientific computing/simulations that are critical to DOE missions and federal efforts. It is intended to improve the utilization of high-performance computing platforms and also facilitate gains in productivity by reducing model redevelopment and/or porting efforts for execution on larger numbers of processors. In addition to DOE, the technology developed as part of this project could potentially be applied to scalable scientific computing efforts in agencies such as the Defense Advanced Research Projects Agency's High Productivity Computing Systems (HPCS) program.

\section{Results and Accomplishments}

We have made progress along all the four tasks originally planned. We have an initial, backward-compatible framework for a subset of the MPI interface extended to support asynchronous, speculative execution. We have demonstrated the feasibility of scalable, asynchronous collective communications and have shown runtime performance that is very competitive with native synchronous MPI implementations on Cray XT4 up to 16,384 cores. We have made inroads into reversible computing techniques applied to the scientific computing domain, with insights on basic linear algebra routines in the reversible computing context, 
essential for efficient speculative execution. We have also demonstrated the significance of performance gains of reverse computation vs. checkpointing in reversing basic linear algebra services (BLAS), especially for those due to caching effects. We are developing RBLAS, a reversible version of the widely used BLAS interface. We also have demonstrated scalability of our time warp-based speculative engine with synthetic benchmarks up to 8,192 cores, which, to our knowledge, is the first of its kind in the world. We have also started a systematic approach to testing and benchmarking the performance benefits of asynchrony and barrierless speculative execution. This is being done in a linear algebra-based formulation of the finite difference time domain (FDTD) method, simulating phenomena such as diffusion and electromagnetic wave propagation in large-scale military wireless radio networks.

\section{Publications}

Perumalla, K. S. 2007. "Symposium on Asynchronous Methods in Scientific and Mathematical Computing 2007," in Proceedings of IEEE/ACM/SCS International Workshop on Principles of Advanced and Distributed Simulation (PADS), San Diego, CA, June 2007. www.pads07.org/asym.

Perumalla, K. S. 2006. "A Systems Approach to Scalable Transportation Network Modeling." Winter Simulation Conference, December 3-6, 2006, Monterey, CA, USA.

Perumalla, K. S. 2006. "Parallel and Distributed Simulation: Traditional Techniques and Recent Advances." Winter Simulation Conference, December 3-6, 2006, Monterey, CA, USA.

Perumalla, K. S. 2007. "Scaling Time Warp-based Discrete Event Execution to $10^{4}$ Processors on Blue Gene Supercomputer." ACM International Conference on Computing Frontiers, May 7-9, 2007, Ischia, Italy.

\section{6: Development of a Global Advanced Nuclear Fuel Rod Model}

Srdjan Simunovic, Larry J. Ott, Phani Nukala, Kevin Clarno, Ian Gauld, Theodore Besmann, and Charles Weber

\section{Project Description}

Advanced nuclear fuel is the primary technology required for the realization of new nuclear reactor technologies, including transmutation fuel designed to consume spent fuel and nuclear weapons material. Central to the development of advanced nuclear fuels with enhanced reliability and accident performance, extended burnup, ease of fabrication, capability to contain significant fractions of minor actinides, and reprocessing is the need to develop a fundamental understanding of the materials involved. The objective of the research is to develop the computational framework needed for fine-scale simulation of nuclear fuel, thereby incorporating the relevant multiphysics and chemistry occurring within a fuel pellet/rod during operational conditions. Such a computational framework should be capable of simulating a large-scale, fully three-dimensional model of nuclear fuel elements to the desired resolution dictated by underlying fuel physics/chemistry. The developed model will provide interfaces and mechanisms for coupling reactor fluid thermodynamics, thermo-mechanics, neutronics, fuel chemistry, chemical species transport, and more detailed multiscale physics models. Due to the physical complexity 
of the problem, and need for high spatial and temporal resolution of the model, scalable computing algorithms and approaches that target relevant phenomena at appropriate time and length scales will be developed. The nuclear fuel modeling computational framework will be implemented on ORNL's leadership computing systems.

\section{Mission Relevance}

Presently, comprehensive, fully three-dimensional computer models of a nuclear fuel rod do not exist. Current "legacy" fuel performance tools are entirely empirical; hence, they are not predictive for processes outside of existing design space and databases. Execution of this program will therefore be ground-breaking work in the area of fuel performance modeling. This work is directly relevant to DOE's Global Nuclear Energy Partnership Program, specifically for the development and modeling of advanced reactor fuel systems. The development strategy is to build on ORNL's existing capabilities in fuel performance modeling and leverage advanced computing tools developed under DOE's SciDAC, Advanced Simulation Computing (ASC), and the Global Nuclear Energy Simulator (GNES) projects. The detailed multiscale model of the nuclear fuel rod will provide the framework for incorporating Basic Energy Sciences (BES)funded atomistic and electronic fuel models.

\section{Results and Accomplishments}

During FY 2007, the project's first year, we made significant progress in three areas: (1) we ported solid mechanics and thermal solver on ORNL MPP supercomputers and demonstrated the solver scalability with increasing problem size and processor count, (2) we simulated coupled neutronics-thermal-mechanics problem of nuclear fuel in channel fluid flow, and (3) we developed model generators and data converters for different physics models and data exchange between various nuclear fuel models. The new integral fuel performance code is built by combining components from different physics codes. Open source finite element method (FEM)based codes were used to develop the core thermo-mechanics modules. This code provides the host for other modules required for modeling the nuclear fuel physics. Scalability of solver performance is based on the Hierarchical Domain Decomposition Method (HDDM) solver that will be used across the multi-physics aspects of the model. Fuel and cladding physical properties and supporting physics modules were provided by simulations using the legacy nuclear fuel modeling codes. The legacy codes were also ported to modern computing architectures. Basic components for nuclear fuel chemistry module have been developed. They will be coupled with commercial chemistry modeling libraries to provide the chemical/phase state of the fuel based on localized inventory of fuel/fission products.

\section{Publications}

Simunovic, S. 2007. "Algorithmic/Simulation Opportunities in Modeling of Nuclear Fuel Rods," Computational Engineering and Science Conference (CESC 2007), April 10-12, Washington, DC. (available through the IEEE database).

Thompson, D. W. T., et al. 2007. Thermodynamic Treatment of Uranium Dioxide Based Nuclear Fuel, International Journal of Materials Research, 2007/10, p. 1004-1011.

Thompson, D. W. T., et al. 2007. "Thermodynamic Model for Uranium Dioxide-Based Nuclear Fuel." Int. J. Mater. Res. 98, 1004. 


\title{
00026: Waveguide Entangled Photon Sources for Quantum Information
}

\author{
W. Grice, R. Bennink, P. Evans, T. Humble, and Z. Zhao
}

\section{Project Description}

The rapidly emerging field of quantum information science (QIS) exploits novel features of quantum physics to open up new possibilities in computing and information management. One of the most exciting prospects is the quantum computer, which would simultaneously process superpositions of different bit states and perform tasks intractable on any classical computer. The creation and manipulation of physical quantum bits is the first step in implementing a quantum computer and represents the current frontier of QIS and the ultimate technical goal of this project.

Photonic quantum computing is one of the leading proposals for quantum computing, drawing on decades of experimental techniques for encoding information in the properties of individual photons. Yet current sources for single and entangled photons are unwieldy and often ill suited for quantum computing. This project addresses these shortcomings with the development of an entangled photon source integrated into an optical waveguide device. A quantum optical "chip" provides the ability to engineer a photon source with the requisite properties while providing the integratarbility and scalability necessary for a functional quantum computer. Characterization and performance testing will complement the design and, ultimately, the fabrication of the proposed device.

\section{Mission Relevance}

QIS is a multidisciplinary endeavor. Significant overlap already exists between QIS needs and the missions of DOE, particularly as reflected in ORNL capabilities (computing, quantum optics, quantum dots, nanoscience, materials science, etc.). As QIS matures, there will be a greater need for improved materials, making DOE even better suited to play a significant role. This was recently brought to light in the American Competitiveness Initiative (ACI), in which QIS was highlighted as a national priority and DOE was given the task of "overcoming technological barriers to the practical use of quantum information processing." The development of a waveguide entangled photon source clearly addresses the ACI challenge.

In addition to benefiting DOE, the project is directly relevant to a number of federal programs. The Department of Homeland Security (DTO, NSA, CIA) and the Department of Defense (ARO) are currently funding projects in quantum computing and quantum communication. The development of a compact entangled photon source with spectral and spatial characteristics perfectly tailored for these applications will directly benefit these programs.

\section{Results and Accomplishments}

The Waveguide Entangled Photon Source project has yielded two significant accomplishments to date. First, we have established a connection between transverse momentum entanglement and the spatial mode composition of emitted photons. This result is relevant to the quantum computing community because photonic quantum computing involves interference of photons 
from independent sources, a process that is impacted directly by the spatial quality of the interfering photons.

In experiments carried out toward the end of 2006, we showed that momentum entanglement can be eliminated in photons emitted from a bulk crystal by focusing the pump beam (i.e., by controlling the amount of transverse momentum in the pump). When the pump is properly focused, the photons are not momentum-entangled and, not surprisingly, the photons are emitted into single spatial modes. Because waveguide devices also emit into single spatial modes, it follows that the photons from such devices must also be free of transverse momentum entanglement, thus satisfying one of the fundamental requirements for photonic quantum computing.

A second result is more directly related to down-conversion in waveguides. Starting from first principles, we have developed the theoretical framework for down-conversion in structured environments. Using this theory, we have obtained some important early results. Firstly, we have obtained theoretical proof that a waveguide can be more efficient at producing spatially unentangled photons than a bulk crystal, and have derived the conditions under which this is the case. Secondly, we have analyzed the effect that simple optical resonators (i.e., cavities formed by external or internal reflectors) have on the joint spectrum of photon pairs and on the total pair production efficiency. As hoped, we have found that resonant structures can increase the flux of spectrally unentangled photons. 



\title{
00188: Applications of Ultrafast, Ultraintense Lasers to Radioactive Ion Beam Production and Diagnostics
}

\author{
David R. Schultz, Charles R. Vane, James R. Beene, Daniel W. Stracener, Charles C. Havener,
} Herbert F. Krause, Junjien Liang, Robert W. Shaw, Warren P. Grice, Anatoly Maksimchuk, and Donald P. Umstadter

\section{Project Description}

Enabled by extremely rapid advances in ultrafast, ultraintense laser (UUL) technology, we are pursuing development of innovative methods for laser acceleration of charged particles, particularly electrons, to relativistic energies as a means for producing radioactive ions for nuclear physics research and other applications. Specifically, we are investigating and developing the necessary stages of proof of concept to produce neutron-rich ion beams and diagnostics for those beams, utilizing UUL pulses. In addition, many novel features of these high-power-density light pulses are being explored with respect to enabling applications aiding other key ORNL objectives, such as providing a compact, ultrafast X-ray light source complementing neutron-scattering capabilities for nanophase, chemical, biological, and material sciences.

\section{Mission Relevance}

This work is particularly relevant to the DOE Office of Nuclear Physics, specifically to a program of upgrades to the ORNL Holifield Radioactive Ion Beam Facility (HRIBF) that will continue until an alternative successor to the Rare Isotope Accelerator project takes shape. The addition of a second unstable nucleus production accelerator would significantly expand HRIBF capabilities, and a compact, laser-driven photofission source would fit particularly well as a future element of the facility's R\&D program. DOE, the Nuclear Science Advisory Committee, and various review committees have endorsed upgrading HRIBF, and photofission drivers could substantially extend the lifetime of HRIBF as a world-leading facility. UUL-based technology can also be applied to homeland security as a compact tool for photofission-based interrogation of shipping containers, to the production of isotopes of interest to the National Nuclear Security Administration (NNSA), and for use in radiotherapy and medical diagnostics. Finally, having built a core UUL science and applications team and established laser-driven electron acceleration capability at ORNL, the basis will exist for further spin-off development work to create an ultrafast Thomson X-ray scattering light source that will provide new opportunities for our nanophase, biological, chemical, material, and physical sciences programs supported by the DOE Office of Basic Energy Sciences.

\section{Results and Accomplishments}

During FY 2007 we completed work on a critically important piece of apparatus that enables the final stage of the proposed proof of concept. This is a differentially pumped vacuum chamber designed and constructed to enable pulsed gas jet operation at a repetition rate of ten times per 
second with helium gas plume densities of greater than $10^{19} / \mathrm{cm}^{3}$, as required to achieve plasma density profiles optimized for laser wakefield acceleration of electrons to several 100's of MeV. Two supersonic expansion gas jet nozzle designs were implemented and characterized at ORNL using measured time-dependent electron elastic scattering and optical imaging of the induced helium fluorescence. One nozzle design provides a reproducible gas plume at the laser focus with cylindrical symmetry of $\sim 2 \mathrm{~mm}$ in diameter, and the other produces a plume with a nearly rectangular cross section to achieve a laser-gas interaction length of $\sim 10 \mathrm{~mm}$. Both have $<1 \mathrm{~ms}$ rise times to stable, nearly uniform density, and have been tested at up to $10^{20} / \mathrm{cm}^{3}$ gas density without exceeding the vacuum pressure design limits imposed by laser defocusing in residual background gas. The latter nozzle design enables acceleration of electrons at the DIOCLES UUL facility at the University of Nebraska up to $\sim 500 \mathrm{MeV}$ using $100 \mathrm{TW} 30 \mathrm{fs}$ pulses.

We also completed work to finalize research papers reporting on the second year's work (now published) and initiated reports on the third year's results. In addition, the first proposal for follow-on funding was written and successfully funded, providing a total about $\$ 1 \mathrm{M}$ from the U.S. Department of Homeland Security, Domestic Nuclear Detection Office, to the University of Nebraska - ORNL collaboration. Preparations were also made for proposing follow-on work to the DOE Office of Nuclear Physics to extend development of radioactive ion production for nuclear research via laser-driven photofission.

\title{
Publications
}

Reed, S. A., et al. 2006. "Photonuclear Fission with Quasimonoenergetic Electron Beams from Laser Wakefields.” Appl. Phys. Lett. 89, 231107.

Reed, S. A., et al. 2007. "Efficient Initiation of Photonuclear Reactions Using Quasimonoenergetic Electron Beams from Laser Wakefield Acceleration." J. Appl. Phys. 102, 73103.

Reed, S. A., et al. 2006. "Laser Wakefield Acceleration of High-Quality Electron Beams to 300 $\mathrm{MeV}$ and Efficient Initiation of Photonuclear Reactions." 12th Advanced Accelerator Concepts Workshop, July 10-15, 2006, Lake Geneva, WI, USA.

\section{8: Theoretical and Computational Methodologies and Tools for Second-Generation Integrated Fusion Simulation}

\author{
Raul Sanchez
}

\section{Project Description}

This project intends to lay the basis for achieving more accurate simulations of fusion experiments close to burning-plasma conditions in the near future. This predictive capability will be critical to support design decisions and to operate next-step experiments— such as the International Tokamak Experimental Reactor or (ITER)—or future power-producing facilities. Due to the large disparity of relevant scales present in the problem (over ten orders of magnitude in time scales and around six in spatial scales) direct simulation is simply impossible with present-day resources. Instead, the problem is broken down in smaller pieces that deal with 
reduced ranges of temporal and spatial scales that must be simulated by specialized codes. All these pieces must then be brought together in order to perform a global simulation in a selfconsistent manner that requires the interchange of relevant information among the different codes in an iterative manner. Many of the codes presently available to attack the partial problems are not the most appropriate to play these roles within the integrated simulation framework just described. Also, determining how information must be passed among the different codes while preserving the essential physics is still the object of active research. The current proposal aims at improving the present situation in both regards. First, we will attempt to lay the basis for development of a new fast magneto-hydrodynamical (MHD) equilibrium code able to tackle the complex magnetic topologies (such as magnetic islands or open field lines) that will be relevant in these global simulations. Second, we will explore several promising methods, not presently available, that may act as efficient interfaces among those pieces of the problem. Namely, we will explore the interaction of fast scales (associated to wave-particle interactions and microturbulence) with the slower ones that determine the confinement properties of magnetic confinement devices.

\section{Mission Relevance}

The DOE Office of Fusion Energy Science (OFES) has recognized the need for accurate simulation capabilities with predictive power as one of its top priorities, from which major future initiatives should be expected. The development of a numerical framework as the one described here-in which various specific codes calculate and efficiently transfer each piece of information relevant to carrying out a global simulation-is the main objective of the Fusion Simulation Project (FSP), an ambitious 15-year program being proposed for funding by the DOE Office of Science. Indeed, the main goal of FSP is to "develop a fully integrated capability for predicting the performance of externally-controlled fusion systems, including turbulent transport, macroscopic stability, wave-particle physics and multphase interfaces." When FSP reaches full funding, it is expected to be a $\sim \$ 20 \mathrm{M}$ joint program between OFES and the Office of Advanced Scientific Computing Research (OASCR). The problems addressed by this project may also benefit the development of numerical tools to support the design and operation of the $\sim \$ 4 \mathrm{~B}$ multinational ITER tokamak project, whose U.S. headquarters are located at ORNL.

\section{Results and Accomplishments}

During FY 2007, we have completed the identification of the different algorithmic pieces needed to put together a prototype version of the island MHD solver code (SIESTA). A large part of the effort has been spent in finding the correct discretization of the set of MHD equations that would ensure an optimal convergence of the code. Also, we have carried out a very careful design that would facilitate the future parallelization of the code into ORNL supercomputers. We have also implemented several schemes to improve the convergence and avoid the deleterious effect of small eigenvalues typical of ideal MHD problems. In particular, matrix preconditioning techniques and a simplified Levenberg-Marquardt scheme have been tested an implemented. A prototype version of SIESTA has been tested successfully on several simple tokamak and stellarator problems, and it exhibits very rapid convergence.

Regarding the investigation on new closure methods of interest for MHD simulations, we have studied the possibility of constructing effective closures via fractional techniques from tracer 
particles simulation in both plasma fluid and gyro-kinetic codes. In particular, pressure-gradientdriven, dissipative-trapped-electron and ion-temperature-gradient turbulence have been explored. In the latter type of simulations, the interaction with mean and zonal flows has been studied. The results confirm that these tools are very powerful not only to provide effective expressions that could be fed into codes dealing with slower/larger scales, but also to characterize the detailed dynamics of short/fast fluctuating scales and identify the mechanisms responsible for the onset of scale-free transport characteristics. They seem usable also for validation and verification $(\mathrm{V} \& \mathrm{~V})$ purposes among codes, one of DOE's priorities for the coming years, suggesting a meaningful role in the context of fluid and gyro-kinetic plasma simulation in massively parallel supercomputers.

\section{Publications}

Calvo, I., et al. 2007. "Continuous Time Random Walks in Periodic Systems: Fluid Limit and Fractional Differential Equations on the Circle." J. Phys. A. 40, 13511-13522.

Calvo, I., et al. 2007. "Fractional Generalization of Fick's Law: A Microscopic Approach." Phys. Rev. Lett. 99, 230603-1

Carreras, B. A., R. Sanchez, and B. P. van Milligen. 2007. "Statistical Characterization of Fluctuations towards Understanding Turbulence-Induced Transport in MagneticallyConfined Plasmas," in "Anomalous Fluctuation Phenomena in Complex Systems," ed. by C. Riccardi, Transworld Research Network, Singapore.

Fernandez-Gomez, I., J. R. Martin-Solis, and R. Sanchez. 2007. "Determination of the Parametric Region in which Runaway Electron Energy Losses Are Dominated by Bremsstrahlung Radiation in Tokamaks." Phys. Plasmas. 14, 72503

Fu, G., et al. 2007. "Ideal Magnetohydrodynamic Stability of the NCSX." Fusion Sci. Technol. 51, 218-231.

Martin-Solis, J. R., et al. 2006. "Enhanced Production of Runaway Electrons during a Disruptive Termination of Discharges Heated with Lower Hybrid Power in the Frascati Tokamak Upgrade." Phys. Rev. Lett. 97, 165002.

Mier, J. A., L. Garcia, and R. Sanchez. 2006. "Study of the Interaction Between Diffusive and Avalanche-like Transport in Near-Critical Dissipative-trapped-electron-mode Turbulence." Phys. Plasmas. 13, 102308.

Sanchez, R., et al. 2006. "Renormalization of Tracer Turbulence Leading to Fractional Differential Operators." Phys. Rev. E. 74, 16305.

Sanchez, R., B. A. Carreras, and B. P. van Milligen. 2007. "Radu Balescu and the Search for an Stochastic Description of Turbulent Transport in Plasmas." Ann. Univ. Craiova Phys. 17, 27-43.

van Milligen, B. P., et al. 2007. "Pulse Propagation in a Simple Probabilistic Transport Model." Nucl. Fusion. 47, 189-195.

Woodard, R., et al. 2007. "Persistent Correlations in Self-Organized Critical Systems away from their Critical Point." Physica A. 373, 215-230. 


\section{6: Nanostructured Surfaces from Drawn Materials}

Brian R. D’Urso and John T. Simpson

\section{Project Description}

This research project demonstrated an elegantly simple and relatively inexpensive method of forming complex, ordered, nanoscale surface array structures without the use of photolithography or complex vacuum systems. Previous Oak Ridge National Laboratory research involving glass drawing resulted in micronscale glass cone arrays as well as wire-inglass arrays. The main objective of this research project was to explore and extend various glass drawing techniques with the goal of producing surfaces and structures with nanoscale features. These high-aspect ratio nanostructures are created by combining the well known processes for fabrication of microchannel glass with new materials. The selection of starting materials is a significant challenge due to constraints on thermal expansion, melting point, and biocompatibility. Glass fiber drawing in a custom fiber-draw tower is used to make structured fibers that are fused into a solid composite rod. The composite is sliced, polished, and etched or differentially etched to create the desired surface features. These nanostructures, including nanocone arrays, nanochannel arrays, nanoneedle arrays, nanowire arrays and optically guiding nanocone arrays, cannot be easily fabricated by conventional methods due to the high aspect ratios of the surface features. While many of these structures have multiple potential uses, they all have potential biomedical applications for drug delivery, neural stimulation, or optical probes.

\section{Mission Relevance}

Nanostructured materials and surfaces are relevant to the materials science and technology programs within the DOE Office of Science, particularly with respect to nanotechnology. Our proposed research is creating nanoscale materials with extraordinary properties and will establish new capabilities in producing nanostructured materials through glass fiber drawing at ORNL. The potential micro-electro-mechanical systems (MEMS) and biomedical applications of these structures may benefit the National Institutes of Health (NIH) and the Defense Advanced Research Project Agency (DARPA). In particular, programs in the DARPA Microsystems Technology Office interfacing electronics and insects could benefit from nanowire arrays for neural interfaces. Microneedle arrays could serve as an in-field, minimally invasive drug delivery system for soldiers. We are in pursuit of follow-on funding to continue this work under new sponsorship.

\section{Results and Accomplishments}

We developed and demonstrated five new nanostructured materials and associated material process-fabrication techniques. (1) Two-dimensional glass nanocone arrays having pitches down to $300 \mathrm{~nm}$ have been produced. (2) We developed a new method of fabricating nanochannel arrays that allow for the creation of much higher aspect ratios than typical nanochannel glassfabrication techniques, using inexpensive commercially available glasses. Channels with hole lengths of a few inches and hole diameters of less that $100 \mathrm{~nm}$ have been demonstrated. (3) We have successfully fabricated arrays of nanoneedles consisting of a surface of sharp cones. Each cone contains a hole passing completely through to the back of the substrate that allow gases or 
liquids to be administered via the back plane. These nanoneedle arrays are potentially a new, less invasive, method of drug delivery. (4) We have also successfully fabricated nanowire arrays that could be used for both electron emission and electrode detection. Nanowire arrays having wire diameters as small as $300 \mathrm{~nm}$, and lengths of at least hundreds of microns have been demonstrated. (5) Finally, we have fabricated optically guiding nanocone arrays within a glass disc. Each cone can be thought of as an optical fiber with its tip sharpened. The result is a glass disc containing an array of sharpened optical fibers capable of independently transmitting or receiving light. This unique fiber-optic array structure could be used as a massively parallel light sensor (i.e., optical probe).

\section{9: Transfer of Vertically Integrated Carbon Nanotube Arrays for Sensors and Thermal Management}

Jeremy J. Jackson and David B. Geohegan

\section{Project Description}

Vertically aligned carbon nanotube arrays (VANTAs) are self-supporting carpets of nanotubes grown by chemical vapor deposition (CVD) onto substrates prepatterned with densely packed metal catalyst nanoparticles. Because they are long, continuous nanotubes grown in dense arrays, VANTAs exhibit outstanding thermal, optical, mechanical, and electrical properties. However, their high-temperature synthesis conditions currently prohibit their use in many application areas that are processed at much lower temperatures, including sensors, plastic electronics, or biological probes. Methods of transferring, patterning, and bonding VANTAs to temperaturesensitive supports are desperately needed to enable new explorations of their functionality and new applications of carbon nanotubes in these areas. In this research project, we are developing experimental techniques for the transfer and bonding of VANTAs to enable the exploration of several application areas involving sensors, optical coatings, and thermal management.

\section{Mission Relevance}

VANTAs are highly relevant to DOE and DoD missions because of their energy-management applications. For sensors, they are high-surface area structures for biofunctionalization and long antennas for RF communication. For energy, they may be mass produced for hydrogen storage and catalyst supports for fuel cells and batteries, and their aligned structure appears highly promising as highly thermally diffusive thermal management materials for cooling everything from hydrogen storage tanks to microelectronics. These application areas make these materials unique as highly multifunctional, anisotropic, mechanical, thermal, and electrical materials for both energy and homeland defense.

\section{Results and Accomplishments}

VANTAs were grown on various substrates to explore different methods of transfer to other platforms. Methods were developed to coat the flat top surfaces of the VANTAs with low melting-point metals and metal alloys, then flip them over, and bond them to substrates such as 
glass and silicon. The VANTAs were delaminated either by mechanical or chemical treatments followed by capture and transfer of the films by printing techniques. The demonstration of several low-temperature processing techniques in this project to adhere VANTAs mechanically and electrically to different platforms will enable diverse applications. The flat top of the flipped VANTA (i.e., the original bottom surface) provides a very smooth substrate of opened nanotubes to serve as platforms for gas storage, biofunctionalization, or sensor studies. These continuous, long nanotubes aligned in tall arrays are proven electrical and thermal conductors that can be encased in polymers to make multifunctional composites that are stronger, with higher thermal and electrical conductivity, and, now, with sensing capabilities. The ability to bond and transfer these materials to new surfaces enables a wide variety of new applications from microscale electronic chips to aerospace structural materials. 

Seed Money Fund 



\section{BIOSCIENCES DIVISION}

\section{6: Identification of Protein-DNA Interaction and Protein-Protein Interaction in Single Living Cells Using Optical Nanosensors}

Yisong Wang, Yie Liu, and Tuan Vo-Dinh

\section{Project Description}

Characterizing molecular interactions in the context of a live cell is a significant challenge for postgenomic studies. This proposal aims to develop DNA- and protein-based optical nanobiosensors and methods to detect protein-protein and protein-DNA interactions in single living cells. We are investigating the use of unique optical nanobiosensors that can be inserted into single living cells to detect molecular interactions without disrupting normal cellular processes. The new method will involve DNA- or protein-based nanobiosensor with a direct in vivo sensing capacity. We are also studying molecular interactions with a special emphasis on developing new detection systems in a single living cell. Our objectives are (1) development and application of optical nanobiosensor technology to protein-DNA interactions and (2) development and application of optical nanobiosensor technology to protein-protein interactions. This work will greatly enhance and benefit interdisciplinary approaches to biological research and current nanotechnology programs at ORNL.

\section{Mission Relevance}

This work will benefit programs of DOE, the Department of Homeland Security (DHS), the Department of Defense (DoD) (Defense Threat Reduction Agency [DTRA], Defense Advanced Research Projects Agency [DARPA]), the Department of Justice (DOJ), and the National Institutes of Health (NIH). The NIH has started several initiatives and issued calls for proposals focused on optical technologies for minimally invasive diagnostics of diseases. The NIH has also established a National Institute for Biomedical Imaging and Bioengineering (NIBIB), with biosensor technologies being one of its top funding priorities. The proposed research fits very well with these NIH initiatives. Finally we will also pursue technology transfer opportunities with commercial companies working in sensor technologies.

\section{Results and Accomplishments}

This proposal aims to develop optical nanobiosensors to detect protein-protein and protein-DNA interactions in single living cells. This will allow, for the first time, studies of molecular interaction in the context of functional cell architecture. Since protein-DNA interaction and some of the biologically critical protein-protein interaction take place in the nucleus of a cell, we are focusing our attention on the detection of these molecular interactions in the nucleus. We have devoted extensive effort to investigate methods to improve the reproducibility of nanofibers. The nanofibers were covalently bound with molecules, such as short telomere repeats and telomeric repeat binding factor (TRF2), that are selective to target analyte fluorescent molecules in the nucleus. We are currently modifying and improving our nanofibers, covalent binding procedures, 
and nanofiber insertion techniques through the evaluation of cellular response, such as cell cycle progression and DNA damage response using GFP- $\alpha$-tubulin and Redmonomer-H2B as markers. The protocol we have established is currently being tested in the detection of single molecular interactions in the nuclei of living cells.

\section{Publications}

Gomez, M. V., et al. 2006. "PARP1 Is a TRF2-associated Poly(ADP-ribose) Polymerase and Protects Eroded Telomeres." Mol. Biol. Cell. 17, 1686-1696.

Wang, Y. and Y. Liu. 2006. "Msh2 Deficiency Leads to Chromosomal Abnormalities, Centrosome Amplification, and Telomere Capping Defect." Oncogene. 25, 2531-2536.

\section{2: Generation of Mouse Embryonic Stem Cell Lines To Study MicroRNA Functions through Conditional and Cell Lineage-Specific shRNA Knockdown Approaches}

Yisong Wang, Mitchell Klebig, Yun You, and Edward J. Michaud

\section{Project Description}

MicroRNAs, members of the noncoding small regulatory RNA family, have recently been shown to regulate gene expression during embryonic development, tumorigenesis, virus infection, cell proliferation, death, and angiogenesis. There are 533 microRNAs identified experimentally in humans so far, and only a few of them have been functionally characterized. In this study, we engineered mouse conditional and nonconditional microRNA knockout cassettes and used a novel vector-based shRNA knockdown strategy to conditionally and cell lineage-specifically deplete the expression of specific microRNAs in mouse embryonic stem (ES) cells. ES cell clones deficient in specific microRNA expression can be established to study the effects of those microRNAs on cell proliferation, differentiation, and apoptosis. This work will pave the way for the functional annotation of microRNAs in mammalian cells and thus will facilitate our understanding of the development of cancer and related diseases.

\section{Mission Relevance}

Demonstration of the success in our complete experimental pipeline in conditional, nonconditional knockout cassette engineering, shRNA cloning, expression and mutant embryonic cell line production and verification of expression of the targeted microRNAs in embryonic stem cells will benefit our petition for National Institutes of Health calls, particularly those involved in noncoding RNA studies, such as PAS-07-241, RFA-GM-08-001 and PAR-06209. The microRNA mutant embryonic stem cells generated in this study may be used to study the mechanistic effects of biohazardous or bioterror materials for DOE-sponsored national security programs. This work will also be beneficial to the DOE-sponsored Collaborative Cross program/Mouse User Facility at ORNL and has enabled us to secure a small portion of funding from Battelle Chronic Obstructive Pulmonary Disease Bioinitiative fund. 


\title{
Results and Accomplishments
}

We have constructed a transgenic construct for the mouse miR-17/18/19a/20/19b-1 polycistronic transgene cluster under the control of a K14 keratinocyte-specific promoter in skin epidermis and conditional knockout cassettes for miR-145 and miR-32. We also generated mouse conditional and nonconditional knockout cassettes for the simultaneous deletion of both miR-15 and -16. The engineered doxycycline-regulatable shRNA cassettes for five target microRNA genes (miR$145,192,218-1,218-2$, and 224), as well as constitutive and doxycycline-regulatable transgenic rescuing vectors for four microRNA genes (miR-192, 218-1, 218-2, and 224) have been introduced into ES cells and under selection for stable clones. The stable ES clones can be used to investigate the effect of microRNA knockdown on cell proliferation, apoptosis, differentiation, and transformation. Those mutant microRNA resources will help us not only explore the importance of microRNAs in cellular transformation but also discover new targets for anticancer therapies.

\section{Publications}

Giannone, R. J., et al. 2007. "Dual-tagging System for the Affinity Purification of Mammalian Protein Complexes." BioTechniques. 43, 296-302.

Gomez, M. V., et al. 2006. "PARP1 is a TRF2-Associated Poly(ADP-ribose) Polymerase and Protects Eroded Telomeres." Mol. Biol. Cell. 17, 1686-1696.

Wang, Y. and Y. Liu. 2006. "Msh2 Deficiency Leads to Chromosomal Abnormalities, Centrosome Amplification, and Telomere Capping Defect.” Oncogene. 25, 2531-2536.

\section{0: A Genomic Analysis of Microbial-Mediated Metal Transformation}

\author{
Antony V. Palumbo, Steven D. Brown, and Craig C. Brandt
}

\section{Project Description}

Anaerobic sulfate-reducing bacteria (SRB) are a diverse group of microorganisms that participate in a wide variety of important environmental processes. In addition to their role in the sulfur cycle, SRB are key organisms in several processes that have significant consequences for humans, including production of monomethylmercury (a potent human neurotoxin), bioremediation of metals such uranium, and corrosion of buried iron tanks and pipelines. Unfortunately, the molecular mechanisms of these processes are not well known. To address this problem, as part of this project we constructed a whole genome microarray for Desulfovibrio desulfuricans $\mathrm{G} 20$, a representative member of the SRB and an organism whose genome has been sequenced by the DOE. Microarray technology is an important tool that allows researchers to obtain insights into cellular processes by examining gene expression under various physiological states. Our research has (i) characterized mercury methylation capabilities Desulfovibrio spp.; (ii) constructed a whole-genome microarray for D. desulfuricans G20; and (iii) conducted baseline gene expression experiments on D. desulfuricans G20 and D. vulgaris. 


\section{Mission Relevance}

This research is relevant to the DOE Genomics: GTL and Environmental Remediation Sciences Program (ERSP) programs. Both of these programs are concerned with metal contamination. The fundamental science and physiology of metal transformations is a focus of the GTL program. The environmental transformations and remediation of metals are the major focus of the ERSP program. Both mercury and uranium are of concern to ERSP. Since D. desulfuricans G20 reduces uranium and belongs to a genus that includes members that do methylate mercury or those that do not methylate mercury, it is relevant to the focus of the ERSP program.

\section{Results and Accomplishments}

We obtained and cultured a number of Desulfovibrio species from the American Type Culture Collection (ATCC) and from a colleague, then conducted preliminary mercury methylation assays in $D$. desulfuricans, $D$. vulgaris, and control strains. The results from these preliminary assays indicated $D$. desulfuricans $\mathrm{G} 20$ and $D$. vulgaris did not methylate mercury under the assay conditions, while control species and Desulfovibrio desulfuricans ND132 and Desulfovibrio africanus ATCC 19997 did produce methyl-mercury. In FY 2007, preliminary data that included draft genome sequence for the $D$. desulfuricans ND132 and D. africanus genomes supported a number of proposals. This project provided the tools and preliminary data for a project now funded by DOE ERSP. This project forms a cornerstone of ORNLs Subsurface Science Focus Area concerned with metal contamination and continues our preliminary studies. We recently had a proposal accepted by the DOE Joint Genome Institute Laboratory Science program to provide finished genome sequences for three methylmercury-producing Desulfovibrio spp. Additional opportunities and interactions included construction of the D. desulfuricans microarray that allowed us to seek collaborators at universities and other national laboratories to target additional sources of funding. Several proposals were and continue to be developed using these tools and preliminary data, such as one responsive to National Institute of Environmental Health needs, which will focus on metal (mercury, selenium, and manganese) contaminants and toxicity at Superfund sites.

\section{0: Establishing a Targeted Mutagenesis System in Clostridium Cellulolyticum}

Yunfeng Yang (David) and Zamin Yang

\section{Project Description}

Clostridium cellulolyticum is a model organism for the mesophilic degradation of cellulose, which is critical for bioethanol production as the most abundant organic polymer in nature and largest component of all plant biomass. Cellulose has the potential to be fermented into useful products such as ethanol, butanol, and hydrogen, making it appealing for the production of biofuels. Although it is clear that Clostridium cellulolyticum digests cellulose by secretion of a protein complex, cellulosome, industrial utilization of the bacterium has been greatly impeded by lack of fundamental biological knowledge. To tackle this gap, ORNL is currently working with 
the DOE Joint Genome Institute to sequence and annotate the bacterial genome. The next challenge is to develop a genetic system for mutagenesis so that the sequence information can be leveraged and the key proteins involved in cellulose degradation can be identified and characterized. In this seed money proposal, we will establish a targeted mutagenesis method in Clostridium cellulolyticum. Suicide vectors available in other related bacteria will be engineered to carry appropriate antibiotic markers for Clostridium cellulolyticum and incorporate DNA fragment(s) of a targeted gene. After transforming the manipulated DNA into bacterial cells, it will be forced into the genome by homologous recombination, resulting in disruption of the targeted gene. The successful accomplishment of this work will make it possible to study genes implicated in biofuel production in the native host Clostridium cellulolyticum.

\section{Mission Relevance}

This research directly addresses the national need of basic research to develop biofuels as a major secure energy source. Currently, basic research of bioenergy production is one of the top priorities of the DOE Office of Science. The Genomics: GTL facility plans have recently been modified to reflect an emphasis on biofuels. During the summer, we believe that a solicitation for two bioenergy centers will be released. These are anticipated to be $30-\$ 50 \mathrm{M} /$ year projects with a focus on bioethanol or biohydrogen. Although specific concepts for the ORNL proposal are just now being developed, having unique capabilities with this organism will allow ORNL to propose comparative studies to other organisms. Also, we anticipate that there will be opportunities for individual projects from several DOE offices including BER.

\section{Results and Accomplishments}

As planned in the proposal, we first determined the native antibiotic resistance characteristics of C. cellulolyticum so that the mutant could be selected based on the introduced resistance. We found that $C$. cellulolyticum was sensitive for rifampicin, tetracycline, chloramphenicol/thiamphenicol, erythromycin and gentamycin. Second, we targeted a cellulosome subunit, Cel8C, for disruption in the genome. To this end, an internal fragment of Cel8C was subcloned into a suicidal vector pKNOCK and transformed into C. cellulolyticum by electroporation under anaerobic conditions. After two weeks of incubation at $34^{\circ} \mathrm{C}$, colonies appeared on a solid minimal medium containing cellobiose as the sole carbon and energy source and $5 \mu \mathrm{g} / \mathrm{mL}$ thiamphenicol for antibiotic selection. PCR amplification of the genomic DNA prepared from those colonies yielded bands of expected sizes when a combination of a vectorspecific primer and gene-specific primers were used. Further sequencing of PCR products confirmed that the suicidal plasmid was integrated into the correct site of the genome. Since disruption of Cel8C was expected to inactivate most of the $\mathrm{Cel}$ gene cluster and hence impair or abolish the functionality of cellulosome, the cel8C mutant was examined for its capacity to grow in VM with cellulose as the sole carbon and energy source. Although the wild-type $C$.

cellulolyticum reached stationary phase after 10 days of incubation at $34^{\circ} \mathrm{C}$, the mutant failed to show significant growth, which served as physiological evidence for the disruption of Cel8C. 


\section{2: Destroying Pathogenic Bacteria Using Targeted Nanoparticles}

Mitchel Doktycz, David Allison, Thomas Thundat, Baohua Gu, Wei Wang, and Christopher Rey

\section{Project Description}

Bacteria are a primary cause of human disease and infection. They can adapt, through mutation, to major changes in their environment, such as to exposure to toxic compounds. Consequently, there are an increasing number of pathogens that have developed a resistance to antibiotics. Alternative therapies are needed. Ideally, these therapies should be generally applicable, targeting multiple components, such that naturally selected mutants in the pathogen cannot avoid destruction. In this project, we are pursuing the development of functionalized nanoparticles for the specific targeting and destruction of pathogenic bacteria. This approach targets magnetic nanoparticles to the cell walls of specified bacteria. After targeting, an alternating magnetic field can be used to inductively heat the attached nanoparticles, destroying the pathogen. The use of nanoparticles should result in localized heating, such that only the pathogen in contact with the nanoparticle, and not the surrounding area, would be destroyed. This should minimize, or eliminate, tissue damage, making this technology suitable for medical applications. Additional advantages are the versatility of the technique and its ability to work in any medium. It should be capable of targeting any selected pathogen through the choice of binding agent and should be capable of destroying pathogens anywhere within the human body due to the depth of penetration of the magnetic field. This technology can be developed as an alternative to antibiotic therapy and can find diverse biomedical uses. Establishing the proof of principle of this technology is the goal of the project.

\section{Mission Relevance}

This work is relevant to the Medical Sciences Research program within the DOE Office of Science, Office of Biological and Environmental Research. Further, it is pertinent to DOE programs related to nanoscience and to efforts related to understanding the fate and transport of nanomaterials in the environment. This technology also addresses research needs in national security as a countermeasure to pathogens that are specifically designed to evade antibiotics. Also, the biomedical focus of this work fits the research needs of the National Institutes of Health. The NIH funds both fundamental and applied studies in interfacing nanomaterials to biological systems and to extending research in nanotechnologies for probing and understanding biological processes. Additionally, this research is relevant to several agencies, including DoD, Defense Advanced Research Projects Agency and the Department of Homeland Security, that fund research on biological countermeasures.

\section{Results and Accomplishments}

We completed FY 2007 project goals regarding (1) synthesis of superparamagnetic nanoparticles and aim (2) characterization of magnetic induction heating of nanoparticles. We have synthesized a variety of nanoparticles and assessed magnetic induction heating. A variety of different magnetite and hematite core particles have been prepared and clad with silica. The inductive heating apparatus has been set up, and initial assessment of heating of the bulk solution by the various nanoparticles has been performed. These studies indicate that significant heating 
of the bulk solution can be accomplished. Further, these studies have revealed the importance of using the proper induction frequency. Progress has also been made toward bioconjugation of the nanoparticles, which is part of our goal of targeting and killing bacteria using nanoparticle and inductive heating.

\title{
00451: Nanostructured 3-Dimentional Electrodes for Enzyme Fuel Cells
}

\author{
Abhijeet P. Borole
}

\section{Project Description}

The aim of this study is to develop a novel enzyme-biofuel cell architecture exhibiting high electron transfer capability, high enzyme loading and activity (increased power density), improved catalyst stability (longevity) and low cost. Enzyme electrodes will be prepared using a salt-lyophilization technique to immobilize hydrogenase and laccase on carbon nanomaterials. This environment will increase enzyme stability, proton transport and electron transport and form a nano-structured, three-dimensional, interconnected bioelectrode. This is expected to increase the power density of the enzyme fuel cells as well. As opposed to current (aqueousbased) enzyme fuel cells, this fuel cell concept will work in a gaseous environment. This system will have the added advantage of minimal catalyst poisoning and short circuiting. The bioelectrode concept proposed here can also be applied to develop fuel cells using vapor-phase ethanol and methanol as fuel source.

\section{Mission Relevance}

This project focuses on developing alternate energy production devices and, specifically, electricity production from hydrogen and renewable resources using biofuel cells. This project is relevant to the DOE's hydrogen, fuel cells, and infrastructure development program and potentially to the bioenergy program. Defense programs may benefit from this project due to its potential to develop portable energy production devices suitable for field application.

\section{Results and Accomplishments}

The project was initiated in the last quarter of 2007. Enzyme fuel cells (EFCs) were designed using laccase-based biocathode and Pt-based anodes. The EFCs were built in two sizes, $1 \times 1 \mathrm{~cm}$ and $3 \times 3 \mathrm{~cm}$. Carbon felt $(\mathrm{CF})$ was used as the backbone material for the cathode electrode. Enzyme was deposited on the CF with or without salt and an electron mediator. Power density was measured at room temperature and in a gas phase mode and found to be on the order of 280 $\mathrm{mW} / \mathrm{m}^{2}$. The use of hydrophilic three-dimensional CF as backbone with limited water content in the anode chamber allows access of the enzyme-coated electrode to air. The CF was further modified with carbon nanotubes followed by enzyme immobilization. This increased the power output to $490 \mathrm{~mW} / \mathrm{m}^{2}$. It was determined that the anode side resistance may be controlling overall power output. Further work is under way to improve the design of anode electrodes using a serpentine flow path. The resistances in EFCs will be assessed in detail using electrochemical 
impedance spectroscopy. This will enable identification of the individual resistances and identify the barriers to proton and electron transfer, enabling improved design and higher power output.

For development of bioanode, the enzyme hydrogenase is being purified from $R$. eutropha. The growth of the organism has been initiated. It will be produced in a $15 \mathrm{~L}$ bioreactor and then purified. 


\title{
00426: Mapping Carrier Distributions and Photovoltaic Activity in Nanophase Materials by Electrical Dissipation Microscopy
}

\author{
Sergei V. Kalinin and Stephen Jesse
}

\section{Project Description}

Understanding the fundamental mechanisms linking the operation of photovoltaic devices to their microstructure can opens pathways to technological advances that will circumvent bottlenecks that currently limit device efficiency. The progress requires the development of capabilities to image the operation of photovoltaic devices, specifically the spatial and electronic structure and photoinduced carrier dynamics, in real space. We propose a novel approach for noninvasive measurements of carrier concentration on the nanoscale using electrical dissipation. Scanning probe microscopy (SPM) offers an approach for probing energy dissipation through detection of the quality factor of the cantilever interacting with surface through the localized tip. Until recently, these measurements were hindered by the fundamental physics of SPM; namely, the dynamic behavior of the cantilever is described by three independent variables (amplitude, resonant frequency, Q-factor), while only two (amplitude and phase) are measured by conventional SPM at a single frequency. Here we propose to develop a band-excitation method for electrical dissipation imaging - a paradigm shift in SPM technology based on nonsinusoidal excitation signals that have a specified spectral density in a given frequency range. The spatial resolution, sensitivity, and calibration of the electrical dissipation SPM will be ascertained using a semiconductor sample with different dopant densities (dopant grating). The method will be used for probing the photovoltaic activity of nanotube based devices, in particular, the identification of individual single-walled nanotubes, determination of their electronic structure and probing their photovoltaic behavior. This data will provide the guidelines for nanotube-based organic photovoltaic (OPV) optimization.

\section{Mission Relevance}

Fundamental and applied research in photovoltaic energy generation is one of the highest priorities to DOE and is a central component of the President's "Solar America" initiative. A novel tool capable of probing carrier distribution in photovoltaic devices in operandi, will provide a key characterization capability for Basic Energy Sciences and Energy Efficiency and Renewable Energy programs. If successful, this method can be implemented on multiple SPM platforms, providing immediate and tangible benefit for energy-related programs. Given the current political and economic situation, long-term research in fundamental and practical aspects of solar energy conversion can be expected to be a high priority for the foreseeable future. Beyond mapping carrier dynamics in semiconductor materials, this work will have a widereaching impact on SPM studies of dissipation processes on the nanoscale and dissipation structure-properties relationship. Furthermore, it is applicable to sensor technologies for aqueous or liquid environments and will be an immediate benefit for the Defense Advanced Research Projects Agency and the Department of Homeland Security. 


\section{Results and Accomplishments}

We suggested and implemented a new family of SPM techniques in which the cantilever is excited and the response is recorded over a band of frequencies simultaneously. This approach allows rapid mapping of the full dynamic response of the system, overcoming the limitations of traditional SPMs based on sinusoidal excitation signals. This band excitation (BE) SPM allows rapid acquisition of the frequency response at each point in an image and, in particular, enables the direct measurement of energy dissipation. The applicability of the BE approach is demonstrated for mapping energy dissipation in magnetic force microscopy (MFM), mechanical and electromechanical probes, including loss processes during ferroelectric domain formation, and the evolution of dynamic behavior of the probes during force-distance curve acquisition. These examples illustrate the universality of the BE method, which can be used as an excitation and control method in all ambient and liquid SPM methods, including standard intermittent mode topographic imaging, magnetic imaging by MFM, electrical imaging by Kelvin probe and electrostatic force microscopies, acoustic imaging by atomic force acoustic microscopy, and electromechanical imaging by piezoresponse force microscopy. In these techniques, BE allows direct measurement of previously unavailable information of energy dissipation in magnetic, electrical, and electromechanical processes. 


\section{4: Development of ZnO Light Emitting Diodes Utilizing Pulse Thermal Processing}

Jun $\mathrm{Xu}$, Ronald D. Ott, and David Norton

\section{Project Description}

DOE has been pursuing the Next Generation Lighting Initiatives for achieving white light efficacy of more than 200 lumens per watt for future lighting sources. The potential for advancement toward the initiative goal is substantial since the efficacy of current state-of-the art light-emitting diodes (LED) is only about 68 lumens per watt. We believe that ZnO-based LEDs have the potential to reach the target goals because of the high exciton binding energy of $\mathrm{ZnO}$ and the ability to control the $\mathrm{ZnO}$ nanostructures. However, a crucial challenge in developing $\mathrm{ZnO}$ LED is p-type doping of $\mathrm{ZnO}$. To overcome this challenge, we proposed to utilize a unique pulse thermal processing (PTP) technique available at ORNL for generating p-type carriers in $\mathrm{ZnO}$. In this Seed project, we have synthesized $\mathrm{ZnO}$ films mixed with nitrogen dopants ( $\mathrm{ZnO}: \mathrm{N})$, activated the dopants using PTP technique, and characterized the films using photoluminescence and Hall Effect measurements. Photoluminescence results show both acceptor-bound exciton $\left(\mathrm{A}^{0} \mathrm{X}\right)$ and donor-acceptor pair (DAP) emissions after pulse thermal processing of nitrogenmixed $\mathrm{ZnO}$, indicating strong conversion of nitrogen dopants into acceptors. Hall Effect measurements are not currently conclusive because the Ohmic contacts of electrodes with the film produced some artifacts. With the current available data, we cannot prove conclusively that p-type $\mathrm{ZnO}$ has been generated using the PTP technique, but it is very clear that this method is very effective for generating $\mathrm{ZnO}$ acceptors, which are crucial elements of p-type $\mathrm{ZnO}$. We will pursue future funding with these promising results.

\section{Mission Relevance}

The U.S. Congress has allocated \$350 million for FY 2007-2013 (P. L. 109-58, Section 912) to support DOE's Next Generation Lighting Initiatives. The goal is to increase performance of light-emitting diodes (LED) to more than 200 lumen/watt by 2025. We intend to contribute to this goal by developing new and better technology in solid state lighting (SSL) devices based on $\mathrm{ZnO}$ LEDs. DARPA is another agency which is interested in highly efficient LEDs.

\section{Results and Accomplishments}

We have accomplished the following: (1) synthesis of $\mathrm{ZnO}: \mathrm{N}$ films, (2) activation of the nitrogen dopants using thermal pulses generated at PTP facility, and (3) characterization of processed $\mathrm{ZnO}: \mathrm{N}$ films using photoluminescence and Hall Effect measurements. Nitrogen-mixed $\mathrm{ZnO}$ films were grown on silicon wafers using a pulsed laser deposition method with surface temperatures of $400^{\circ} \mathrm{C}$ and $500^{\circ} \mathrm{C}$. These temperatures are lower than those used in conventional doping processes in order to prevent loss of nitrogen via $\mathrm{N}_{2}$ formation. As-synthesized films were then processed in the pulse thermal processing (PTP) facility at ORNL. The time profile of the thermal pulses was controlled to execute a long preheating period at low power, followed by 
a short activation step at high power. As-grown and PTP-processed samples were analyzed using photoluminescence (PL) at $8.8 \mathrm{~K}$. The processed samples have also been evaluated with the Hall Effects method. PL results show both acceptor-bound exciton $\left(\mathrm{A}^{0} \mathrm{X}\right)$ and donor-acceptor pair (DAP) emissions after pulse thermal processing of nitrogen-mixed $\mathrm{ZnO}$, indicating strong conversion of nitrogen dopants into acceptors. Hall Effect measurements at this time are not conclusive because of artifacts introduced by the Ohm contacts of the electrodes with the film. PTP has been proven to be very effective for activating nitrogen into p-type carriers for nitrogenmixed $\mathrm{ZnO}$ grown at $400^{\circ} \mathrm{C}$, although it is less effective for nitrogen-mixed $\mathrm{ZnO}$ grown at $500^{\circ} \mathrm{C}$ because of higher probability of $\mathrm{N}_{2}$ formation and diffusion out of the film. This is consistent with our predictions in the proposal. The primary accomplishment for this project is the demonstration of the proof-of-principle: p-type carriers in $\mathrm{ZnO}$ can be generated by substitution of lattice oxygen with nitrogen through the non-equilibrium PTP process.

Follow-on funding will be pursued from DOE Next Generation Lighting Initiative. The goal of the initiative is to cut lighting energy by $50 \%$ by Year 2025 , which requires the white light efficacy of LED devices to be more than 200 lumens/watt. To achieve this goal, the Congress has appreciated \$350 million for 2007-2013 (P. L. 109-58, 912). Our ZnO LED approach should allow us to obtain funding from both BES and EERE of DOE and contribute to this initiative.

\section{4: Selective Electrochemical Oxidation of Water for Treatment of Ischemic Diseases and Other Applications}

Elias Greenbaum, Charlene A. Sanders, Barbara R. Evans, Hugh O’Neill, Vilmos Kertesz, and Mark S. Humayun

\section{Project Description}

Diabetic retinopathy is a disease characterized by deprivation of oxygen (ischemia) to the retina, resulting in impaired retinal function and eventual retinal photoreceptor loss. Clinical studies have shown that oxygenation of the retina can have therapeutic use in retinal vascular occlusive diseases. This project proposes the electrochemical oxidation of saline solution for the production of oxygen $\left(\mathrm{O}_{2}\right)$, deliverable to anoxic tissue, without the formation of potentially toxic free chlorine. The strategy to accomplish this goal will be approached in three ways: (1) design and construct apparatus to measure levels of free chlorine and oxygen in saline solutions charged with anodic electrical pulses from platinum (Pt) sphere electrodes; (2) optimize chlorine suppression by selective application of kinetic pulse profiles, varying amplitude, dwell time, and repetition rate, or rapid pulse phase reversal; (3) test the effects of surface-modified Pt electrodes and PAN (polyacrylonitrile) carbon electrodes on $\mathrm{O}_{2}$ production efficiency. Proof of principle in this project is the avoidance of chlorine formation when a salt solution is electrolyzed to form physiologically significant concentrations of oxygen. If this objective is realized, it will have important implications for the treatment of retinal ischemia, applicable to all forms of systemic ischemic vascular disease. 


\section{Mission Relevance}

This work falls within the scope of the Advanced Biomedical Technology Research program at the DOE Office of Biological and Environmental Research. It reflects the goal of DOE to utilize national laboratory expertise and facilities to develop sophisticated and sensitive biomimetic devices for medical applications. It could also generate funding opportunities from the Defense Advanced Research Projects Agency, the National Institutes of Health, and private agencies interested in biomedical research and applications.

\section{Results and Accomplishments}

We have made considerable progress in this project with the FY 2006 budgeted funds. A closedsystem gas flow electrolysis apparatus has been constructed and is fully operational. Nitrogen carrier gas sparges through phosphate-buffered saline (PBS) in a water-jacketed electrolysis flow cell, removing gaseous products of electrolysis downstream to an oxygen galvanic sensor and a hydrogen Figaro sensor. A multichannel systems stimulus generator drives the Pt sphere electrodes in the flow cell. An assay for free chlorine, involving spectrophotometric analysis of the oxidation of ascorbate by chlorine, has been used to monitor thresholds of chlorine production in the cell during pulse stimulation. An $800 \mu \mathrm{A}$ anodic pulse, $400 \mu$ s dwell time, produced $0.25 \pm 0.01 \mu \mathrm{mol} \mathrm{O}_{2} / \mathrm{h}$ and $0.05 \pm 0.008 \mu \mathrm{mol}$ chlorine/h over the $4 \mathrm{~h}$ stimulation period. Decreasing the dwell time from $400 \mu$ s to 300 and $200 \mu$ s decreased $\mathrm{O}_{2}$ and chlorine incrementally. At 160 and $100 \mu \mathrm{s}$, chlorine was entirely absent from the PBS, but $\mathrm{O}_{2}$ was measurable at $0.09 \pm 0.01$ and $0.06 \pm 0.003 \mu \mathrm{mol} / \mathrm{h}$, respectively. This presents first-stage proof of principle that stimulation pulse profile can be manipulated to oxidize water at the anode before negatively charged chlorine ions can reach the charge exclusion zone at the negatively charged metal electrode. The partitioning of oxidizing equivalents between oxygen and chlorine formation is subject to electrochemical control.

These results have far-reaching implications for applications in neural electrode science and biomedical engineering research. In artificial sight experiments, electrodes have been implanted on the retina to activate retinal neuronal cells without accumulation of harmful electrochemical reaction byproducts. This demonstrates the feasibility of electrode-driven $\mathrm{O}_{2}$ production in the eye for treatment of ischemic retinopathy.

\section{4: Taming Photosynthesis Regulation through Genomics for Direct Synthesis of Ethanol from Carbon Dioxide and Water}

James W. Lee, Barbara R. Evans, and Gary Van Berkel

\section{Project Description}

This project is designed to answer an important scientific question: Is it possible to tame the photosynthesis regulation mechanism in such a way that the reducing power (NADPH) and energy (ATP) acquired from photosynthetic water splitting and proton gradient-coupled electron transport process will be used for immediate synthesis of ethanol $\left(\mathrm{CH}_{3} \mathrm{CH}_{2} \mathrm{OH}\right)$ directly from 
carbon dioxide $\left(\mathrm{CO}_{2}\right)$ and water $\left(\mathrm{H}_{2} \mathrm{O}\right)$ ? We will test this scientific hypothesis by accomplishing the following three tasks: (1) synthesis of designer genes to introduce phosphoglycerate mutase and enolase into algal chloroplast; (2) genetic transformation and screening of transformed cells; and (3) expression of the designer phosphoglycerate mutase and enolase genes to test the hypothesis. Successful accomplishment of this project would represent fundamental breakthroughs that could lead to a profound enabling technology for photosynthetic production of ethanol directly from $\mathrm{CO}_{2}$ and $\mathrm{H}_{2} \mathrm{O}$ that will help ensure our nation's energy security.

\section{Mission Relevance}

This research is directly relevant to DOE national energy security missions. The objective of this project is to demonstrate the proof of the concept focusing on the first two enzymes (phosphoglycerate mutase and enolase) of the envisioned ethanol-production pathway that could serve as a new clean energy (ethanol) resource. Successful accomplishment of this project would represent fundamental breakthroughs that could lead to a profound enabling technology for photosynthetic production of ethanol directly from $\mathrm{CO}_{2}$ and $\mathrm{H}_{2} \mathrm{O}$. Therefore, this project is clearly aligned with the President's Advanced Energy Initiative (biofuel ethanol) and supports the DOE Bioenergy Center's mission. This project could also benefit other federal agencies such as the Environmental Protection Agency (EPA) and the U.S. Department of Commerce, since the envisioned photosynthetic ethanol-production technology could lead to a new clean energy industry (creating new opportunities for the economy) that could not only help ensure our nation's energy security, but also help to protect the Earth's environment from the dangerous accumulation of $\mathrm{CO}_{2}$ in the atmosphere.

\section{Results and Accomplishments}

During this reporting period (FY 2007), we completed the DNA sequence design for a set of five designer genes to encode genetic insertion of designer-pathway enzymes, including phosphoglycerate mutase and enolase into algal chloroplasts. These designer genes have now been physically synthesized in collaboration with a biotechnology company, according to the DNA sequence design. The DNA sequence design for the designer genes was accomplished by identifying an appropriate promoter, a proper chloroplast-targeting sequence, and the coding sequences for the phosphoglycerate mutase and enolase enzymes. The identification of these DNA sequences was achieved through searching the GenBank databases and the Chlamydomonas genome databases. The synthesis of the designer genes has just been completed. The ligation of the designer genes together into the plasmid vector is now in progress. As soon as the designer genes are ligated into the plasmid vector and their sequences are verified by DNA sequencing, we will deliver them into the Chlamydomonas host strain for expression of the designer phosphoglycerate mutase and enolase genes to test the hypothesis for photosynthetic production of ethanol from carbon dioxide and water. In preparation for the transformation of the algae and subsequent selection and assays, pertinent characteristics of the host strain were examined. Since these algae are known to produce low amounts of ethanol by fermentation under dark and anaerobic conditions, the baseline ethanol production rate of the host strains was examined under the same test conditions that will be employed to detect expression of the recombinant photosynthetic ethanol construct. The background rate of ethanol production under the anaerobic light-dark cycles that are used in the flow system experiments is very low, and increased ethanol production from expression of the recombinant genes will be readily 
detectable. The effectiveness of the selectable marker arginosuccinate lyase in the target host strain, the arg2 mutant CC-48, was verified and gamete autolysin was prepared for utilization in the algal transformation protocol.

\section{5: Synthesis of Polymeric Materials for Blue-Light Emitting Diodes}

Reza Dabestani, Kunlun Hong, and Gilbert M. Brown

\section{Project Description}

Organic light emitting diodes (LED) capable of generating ultraviolet and blue light are in high demand and have long been sought for electronic device applications. One of the approaches in this challenging endeavor has focused on target molecules that can undergo a sequential onephoton, low-energy absorption process to create higher energy excited singlet states (energy upconversion) that can emit the required blue light. Quantum efficiency for energy transfer (e.g., intersystem crossing from singlet to triplet and/or triplet to triplet) must be close to unity to render this approach feasible for the light-harvesting process. One such system that has recently been reported involves triplet sensitization of organic molecules (e.g., anthracene) by a ruthenium(II) complex, a very efficient triplet sensitizer, via a metal-to-ligand charge transfer (MLCT) band. Low energy excitation of ruthenium(II) complex by green light leads to efficient formation of ruthenium(II) complex MLCT triplet state, which can transfer its energy to anthracene to form an anthracene-excited triplet. Annihilation of two anthracene triplets can lead to an excited singlet (which emits blue light) and ground state anthracene. We propose to design novel polymeric materials that contain a triplet sensitizer (e.g., ruthenium(II) complex) and a triplet acceptor in a random fashion to induce efficient up-converted energy transfer for generating blue light under solid state conditions. Our strong background in polymer synthesis and characterization coupled with our expertise in spectroscopic techniques (photochemical and electrochemical) will enable us to provide the proof of principle to demonstrate the feasibility of this technology.

\section{Mission Relevance}

Organic entities capable of generating blue light using low power light (or voltage) sources are in high demand by the electronic industry. The proposed research offers great opportunity for the proof of principle in this area and the development of the first generation of blue-light emitting solid-state devices via up-converted energy transfer. Our proposed work is relevant to the research programs funded by DOE's National Center for Solid State Lighting Research and Development and Basic Energy Sciences programs. We also anticipate great interest by the electronic industry upon successful proof of principle for the continuation of this effort to the development stage. 


\title{
Results and Accomplishments
}

Styrene based copolymers containing the sensitizer (Ru-complexed bipyridine, $\mathrm{Ru}(\mathrm{bpy})_{3}{ }^{++}$) and the acceptor (diphenylanthracene, DPA) have been successfully prepared by two different methods: (a) reversible addition fragmentation chain transfer (RAFT) and (b) free radical initiator Azobisisobutyronitrile (AIBN), both controlled free radical polymerization processes. Detailed analytical characterizations of both copolymers confirm the presence of bipyridine (bpy) and diphenylanthracene (DPA) in the polymer matrix ( $3 \%$ by weight). Complexation of the bpy moieties present in the polymer matrix to ruthenium metal was then carried out on both copolymers, and the resulting Ru-complexed copolymers were analyzed by UV-Vis and fluorescence spectroscopy to confirm the presence of sensitizer (Ru(bpy) ${ }_{3}{ }^{++}$) and acceptor (DPA) in the polymer matrix. Detailed spectroscopic investigation of copolymer films prepared by spin coating show that triplet energy transfer from the sensitizer (when excited with green light at 510 $\mathrm{nm}$ ) to the acceptor (DPA does not absorb the $510 \mathrm{~nm}$ light) takes place in the polymer matrix leading to blue-light $(430 \mathrm{~nm})$ emission from the acceptor via triplet-triplet annihilation route. These results clearly support the proof of principle that energy up-conversion is a viable path in a polymer matrix that embeds both the sensitizer and the acceptor.

\section{0: Probing the Molecular Interface of Cellulose and Lignin in Biomass}

\author{
Barbara R. Evans, Hugh M. O’Neill, Volker Urban, and Dean Myles
}

\section{Project Description}

Plant-derived cellulosic biomass offers an abundant, renewable feedstock for production of fuels and chemicals, but fundamental understanding of its molecular-level chemical and physical structures is required to improve the efficiency of biomass conversion. Neutron scattering techniques could potentially provide such molecular structural and three-dimensional conformational information. This preliminary study was carried out to test the concept that the component polymers cellulose and lignin purified from biomass could be used to define the neutron scattering signals specific to each component and distinguish them in pretreated biomass samples. Techniques for sample preparation and analysis that have been successfully employed with proteins and other biological materials were used. These are based on use of mixtures of deuterated and regular water as a solvent, because controlling the deuteration level of the solvent allows tuning sample contrast and enables selective visualization of molecular regions that differ in their respective average isotopic composition. Small-angle neutron scattering (SANS) was carried out on the prepared samples, and the resultant data was analyzed for features reflecting conformation and morphology. The scattering signals from the purified components were compared to those from pretreated biomass.

\section{Mission Relevance}

The development of high-resolution imaging techniques for characterizing and understanding the conversion of biomass feed stocks to fuels was described as a priority for future development in 
the DOE Joint Biofuels Roadmap released by the U. S. Department of Energy and in the Genomes to Life Bioenergy Research Center White Paper released in August 2006. The interface of the neutron science capabilities at ORNL with the new bioenergy center will provide exciting research possibilities that will revolutionize biomass production and conversion. During the preparation, revision, resubmission, and eventual funding of this preliminary research project, contacts and discussion with colleagues in the fields of bioenergy, computer modeling, and neutron science expanded the initial concept and focused on defined problems in biomass deconstruction. This preliminary research process resulted in DOE programmatic funding to develop dynamic neutron scattering assisted by molecular modeling and simulation for real-time observation of biomass deconstruction.

\section{Results and Accomplishments}

The planned scattering experiments were successfully carried out and the results are being prepared for publication. The match points, the ratio at which the polymer will be indistinguishable from solvent in a range of $\mathrm{D}_{2} \mathrm{O} / \mathrm{H}_{2} \mathrm{O}$ ratios, were calculated for lignin and cellulose based on the chemical structures and predicted accessibility in exchange equilibrium with the protons/deuterons of the $\mathrm{H}_{2} \mathrm{O} / \mathrm{D}_{2} \mathrm{O}$ solvent. Phase contrast series with known ratios of deuterated water were then prepared using two commercial preparations of purified lignin and microcrystalline cellulose (Avicel). Neutron scattering patterns were collected with the BIOSANS instrument located at the ORNL High Flux Isotope Reactor. The SANS pattern from sulfonated lignin showed a signature corresponding to dispersed 1.3-1.5 nm particles, with a contrast match point at $43.2 \% \mathrm{D}_{2} \mathrm{O}$ in agreement with the calculated value of $43.8 \% \mathrm{D}_{2} \mathrm{O}$ for fully accessible lignin. The SANS pattern from carboxylated lignin is dominated by structures one or more orders of magnitude larger than the individual lignin molecules observed in sulfonated lignin. The contrast match point for Avicel was determined to be $40+/-2 \% \mathrm{D}_{2} \mathrm{O}$, close to the average of accessible (44.9\%) and inaccessible $(33.6 \%)$ cellulose. At $40 \% \mathrm{D}_{2} \mathrm{O}$, coherent scattering at low angles virtually vanishes, indicating the coexistence of accessible/inaccessible cellulose at the local scale of $1 \mathrm{~nm}$. Preliminary analysis suggests morphology of crinkled fibrils with diameters of 1.5 to $2.0 \mathrm{~nm}$. The scattering pattern obtained with steam-exploded wood was more complex than that of simple addition of lignin and microcrystalline cellulose, but cellulose fibrils could be distinguished.

\section{3: Monte Carlo Simulation of Ion Trajectories in Ion Mobility Spectrometry}

Jun $\mathrm{Xu}$ and Yuan Liu

\section{Project Description}

Ion mobility spectrometry (IMS) detectors have been widely used for detecting trace explosives, chemical warfare agents, and toxic industrial compounds. Nevertheless, many problems remain, including a high rate of false alarms and limits in identifying emerging threats. New IMS instruments must overcome these limitations with improved sensitivity, reliability and a greater library of detectable agents. To help address these limitations, simulations of ion trajectories are 
needed. However, no software is available for reliable and specific simulation of ion mobility spectrometry data. In this Seed project, we have developed a program incorporated into SIMION, an electrostatic lens analysis and design program, for Monte Carlo simulation of ion trajectories in IMS instruments (MCSIMS). This project is unique because we developed algorithms based on elastic collisions between ions and gas particles in the center-of-mass system. This software is capable of calculating the mean-free-path of ions, and it takes into account parameters of IMS instruments, such as gas pressure, temperature, ion and drift gas masses, flow direction, and rate of drift gas. The software has been used to calculate ion mobility spectra of various chemicals, predict drift times, and ion transport efficiency. This information can be used to understand separation of analytes from interferents in IMS instruments, leading to reduction of false alarm rates.

\section{Mission Relevance}

Ion transport in air at atmospheric pressure is the fundamental process that ion mobility spectrometers use for detection of explosives, drugs, and chemical and biological warfare agents. Nevertheless, the current IMS systems have limitations that prevent the technology from addressing a variety of emerging threats. The predominant problems with existing IMS detectors are their high false alarm rate and the limitation of the number of detectable threat agents. This simulation software will provide a better understanding of ion trajectories and foster the development of more reliable IMS instruments. Successful demonstration of MCSIMS software will enable us to contribute to DHS, DOE, and DoD applications.

\section{Results and Accomplishments}

We have developed a program that works as a component of SIMION that can perform Monte Carlo simulations of ion trajectories in ion mobility spectrometry instruments (MCSIMS). The MCSIMS program determines ion kinetic energy and scattering angles using a hard-sphere model for individual collision between an ion and a gas particle in the center-of-mass system. The calculation addresses specific issues associated with ion mobility spectrometry instruments. Characteristic parameters in IMS, including mass of ions, drift-gas pressure, temperature, and drift field, can be input into the codes. Ion trajectories obtained from the codes can reveal ion locations, drift time, and ion transport efficiency.

An IMS drift channel, which consists of ten L-shape electrodes and one Faraday plate, has been constructed with SIMION. Drift field, adjustable by the user program ranging from 10 to 1000 $\mathrm{V} / \mathrm{cm}$, have been considered for driving ions to the detector end. In a SIMION simulation, ions travel under a vacuum. In our MCSIMS simulation, ions travel at gas pressures ranging from 7.6 to 760 Torr. Trajectories obtained from our MCSIMS demonstrate that a random walk motion exists in addition to the macro-scale ion drift movement. IMS spectra of 101 ions under gas pressure of 120 Torr and drift bias of $500 \mathrm{~V}$ has been calculated. This simulation shows a resolution of $\mathrm{R}=46$ for the proposed IMS configuration.

Recently, in response to the SERDP FY 2008 funding call, we proposed a new sensor based on the combination of IMS and differential mobility spectrometry (DMS) for monitoring contaminated groundwater in the Superfund sites and 3000 DoD sites. We used this program to investigate the feasibility of the new sensor and presented data from the Seed project in our 
proposal. The peer reviewers gave very positive responses to our simulation data. As a result, we have obtained \$1.004K in FY 2008 funding from SERDP.

\title{
00457: Turbopump Concentration of Heavy Atoms and Molecules
}

\author{
William B. Whitten and Peter T. A. Reilly
}

\section{Project Description}

The ratio of output to input pressure of a turbopump increases with the mass of the species being pumped. Our project is to explore this phenomenon for concentration of atoms or molecules with mass greater than nitrogen or oxygen at the input of an environmental mass spectrometer to increase the sensitivity for trace substances in the atmosphere. Substances of interest include the noble gases, krypton and xenon, volatile organic compounds, chemical warfare agents, explosives, and drugs. The experiments will use a mass spectrometer at the exit of a turbopump to determine the concentration of heavy sample molecules in a carrier gas such as $\mathrm{N}_{2}$. The concentration is expected to occur due to diffusion of the lighter carrier gas to the low-pressure inlet of the turbopump. This low-pressure environment will be maintained by a second turbopump. The pump performing the concentration will probably be operated at a lower than normal rotational speed to permit the selective diffusion of the carrier gas.

\section{Mission Relevance}

Analysis of trace quantities of volatile organic compounds and atomic species in the atmosphere is important to many agencies. Applications range from detection of toxic substances in manned spacecraft by NASA, chemical warfare agents and explosives by the military, and substances in the atmosphere by environmental scientists studying climate change. DOE (NA-22 and NA-24) programs for the detection of the noble gases is relevant to nuclear nonproliferation because these substances are products of nuclear fission. The ability to concentrate the substance of interest can enhance the sensitivity of the analysis and reduce the probability of false alarms.

\section{Results and Accomplishments}

This project has been under way for only 7 weeks. A vacuum chamber has been modified for the experiments. Two Varian V70D turbopumps with separate roughing pumps are connected to the chamber so that one pump can be used for the enrichment process and the other to provide the low-pressure environment at the entrance of the enrichment pump. An ionization gauge monitors the pressure within the chamber and thermocouple gauges monitor the high-pressure outlets of the two turbopumps. A sample gas inlet is installed at the high-pressure side of the enrichment pump, and this volume is also connected to a Polaris-Q ion trap mass spectrometer to determine the relative concentration of the gas at the exit of the enrichment turbopump. Initial experiments will be performed with a mixture of nitrogen to which $1 \%$ sulfur hexafluoride has been added. $\mathrm{SF}_{6}$ was chosen because the detected ion, $\mathrm{SF}_{5}+$, does not react appreciably with residual $\mathrm{O}_{2}$ in the ion trap. A cylinder of the sample gas has been ordered. 



\section{COMPUTATIONAL SCIENCES AND ENGINEERING DIVISION}

\section{7: Discrete Event-Based Simulation of Electromagnetic Wave Propagation in Highly Cluttered Environments}

James Nutaro and Phani Teja Kuruganti

\section{Project Description}

We have developed and validated a model that can rapidly predict the path-loss of a radio channel in a cluttered propagation environment. Our model uses a computationally efficient, discrete-event approximation of the wave equation to rapidly and accurately simulate wave propagation through a digital model of the study site (e.g., DEM or DTED data, VRML or other CAD models, etc.). The radio channel model is intended primarily for use in performance studies of wireless networks.

Wireless network performance in cluttered environments cannot be accurately predicted using empirical models of the network's radio channels. Unlike wired channels that are stationary and predictable, wireless channels interact in complex ways with the environment, and, consequently, are very difficult to analyze. Crude empirical models continue to be used in network performance studies because more accurate and computationally feasible alternatives do not exist. The computationally feasible, site-specific model developed as part of this research project could replace empirical models that are presently used in wireless network simulations.

\section{Mission Relevance}

Computationally feasible, site-specific models of a wireless network are needed by the Army's modeling and simulation programs for the Future Combat System (FCS) and Military Operations in Urban Terrain (MOUT); the absence of a suitable simulation technology is a critical shortcoming. This research project produced a radio channel model that satisfies this need. The precision and accuracy of network models that are used by DoD to study urban combat operations can be significantly improved by integrating our new model with packet-level simulators such as OPNET and QualNet.

\section{Results and Accomplishments}

In FY 2006 we published a paper in TOMACS that describes the mathematical foundations of the model, conducted two validation studies, and published the results of the first validation study. In FY 2007 we prepared and submitted to IEEE Transactions on Antennas and Propagation a publication that describes the results of the second validation study and essential features of the simulation algorithm; this paper has been accepted and will appear in print in January of 2008. We have presented the results of our research to potential Army, Navy, Joint Forces, and industrial sponsors and are pursuing additional funding opportunities. 


\section{5: A Hybrid Diffusion Model Driven by Chemoattractants}

Richard C. Ward, Kara L. Kruse, James J. Nutaro, Barbara G. Beckerman, Oscar Grandas, MD

\section{Project Description}

A hybrid diffusion model (HDM) is being developed to predict complications associated with treatment of vascular occlusive diseases (atherosclerosis) by balloon angioplasty. When complete, the model will simulate the main features of the complex intimal hyperplasia (IH) process that may lead to restenosis of the artery due to vascular remodeling. The model will include discrete simulation of changes in the state of several cell types and their subsequent proliferation and/or migration within the artery wall. The model will also include continuous simulation of the production, diffusion, reaction, and degradation of several chemoattractants and matrix degrading enzymes. This HDM is a novel combination of discrete-event simulation and continuous simulation. Ultimately, the objective is to predict the combination of mechanical and biochemical initial states and changes that push the system balance from a stable state to the potentially lethal unstable state. The team is collaborating with the University of Tennessee Graduate School of Medicine (UTGSM) to gather experimental data to estimate model parameters. The long-term goal is to help physicians predict appropriate treatments to effect desired outcomes in individual patients and provide a baseline model for use in determining the effects of hormone replacement therapy (HRT) on the development of vascular remodeling after balloon angioplasty.

\section{Mission Relevance}

In addition to systems biology applications, the model could be applied to a wide range of problems that are characterized by high nonlinearity and a mixture of continuous and discrete elements that are currently of concern to the DOE. These problems include stability, control, and monitoring of the electric power grid; risk analysis for geologic carbon sequestration; and optimization of advanced combustion systems and nuclear fuel cycles. The HDM approach adopted here will provide general analysis methods and tools that will prove beneficial in many, if not most, of these other research areas of interest.

A goal of the National Institutes of Health (NIH) is to develop computational, predictive models combined with quantitative data to anticipate the normal and disease responses of complex physiological processes, including vascular flow and remodeling. Using the approach developed by this research, a research grant (R01) was submitted to NIH entitled "Integrative Experimental/Computational Model of HRT Effects on Vascular Pathology”, which was submitted on October 5, 2007. A key objective is to use computational modeling to identify key processes and interdependencies determining the effects of estrogen and or progesterone on vascular pathology after injury. Ultimately, the proposed work is to develop a computational modeling tool that can be used to predict critical rate-limiting steps in the pathology and to identify pharmacological interventions anticipated to prevent or hinder vascular remodeling. The HDM serves as a starting point for more extensive models proposed for this grant submission. 


\section{Results and Accomplishments}

A model of cell migration stimulated by a constant biochemical gradient was constructed, and the model verified against established continuum models of cell population migration. The model was enhanced by incorporating a finite difference approximation of a varying biochemical diffusion gradient to simulate the Boyden chamber experiments that are being conducted at UTGSM. Two sets of experimental data were obtained from the Vascular Research Laboratory at UTGSM that will be used to calibrate the model and validate its predictions. Considerable interaction between ORNL and UTGSM was important to the development of the model and experimental protocols. A preliminary object-oriented design concept for modeling the larger IH problem was created. The literature was searched for useful data that could be used in the model. Four student interns helped with these tasks during the summer.

Research sponsored by the Laboratory Directed Research and Development Program of Oak Ridge National Laboratory (ORNL), managed by UT-Battelle, LLC for the U. S. Department of Energy under Contract No. DE-AC05-00OR22725.

\section{Publications}

J. J. Nutaro, et al. 2007. "A Discrete Cell Migration Model," Proceedings of the Society of Modeling and Simulation International (SCS), SCSC'07, San Diego, California, July 15-18. 



\section{4: Smart Tunneling Barriers: A New Concept for Ferroelectric- Based Nonvolatile Random Access Memory}

Vincent Meunier, Marco Buongiorno Nardelli, William A. Shelton, Hans Christen, Ho Nyung Lee, and Sergei V. Kalinin

\section{Project Description}

While computer technology has developed at a tremendous pace, it is not obvious to all users that many important technological developments-including critical changes of technological paradigms - have been needed to achieve current performance. In particular, entering the digital age would not have been possible without huge breakthroughs in the design of random access memory (RAM) chips. RAM is a memory that can be accessed or written to randomly (i.e., any byte or piece of memory can be used without accessing the other bytes or pieces of memory). Until recently, there were two basic types of RAM: dynamic and static. The problem is that both types of RAM are volatile, and no viable technology is currently available for affordable, highspeed, nonvolatile memory. In this project, we are investigating two new paradigms for nonvolatile memory: the molecule-based gated switch, and the asymmetric ferroelectric tunneling element (AFTE). Both systems will provide a viable and more efficient alternative to current technology. We propose to provide a proof of concept using state-of-the-art theoretical and computational methods. We will combine ab initio electronic structure calculation methods that can adequately access the electronic structure of doped nanotubes, complex oxide material, and heterostructures with the non-equilibrium Green's function approach developed at ORNL to address finite-bias transport properties of this type of devices for the first time. During this theoretical effort, we will be in constant communication with experimental groups, which will open the road to explicit implementation of the concept.

\section{Mission Relevance}

This work will benefit the DOE Office of Science in the areas of nanoscale science and technology. This research will provide a much needed link between the first-principles theory and materials preparation, providing clear guidelines for the prediction, design, and synthesis of materials with desired properties. With successful implementation of asymmetric ferroelectric tunneling element heterostructures, the same approach can be used for other multifunctional oxide structures, including ferroelectric field effect transistors and superconductive heterostructures. The results of the study coupled with ORNL's strong program in the growth of multifunctional oxide thin films and heterostructures will also benefit programs of the Office of Naval Research and the Defense Advanced Research Projects Agency.

\section{Results and Accomplishments}

We designed, on the computer, a new type of nonvolatile memory element based on the reorientation of an organic molecule inside a metallic nanowire. The functioning principle is based on quantum confinement effects at the nanoscale. We have developed a full theoretical 
understanding of the functioning of the switching device by introducing the concept of molecular gating. A molecular gate is similar to a conventional gate in a transistor device but differs in the fact that it is not coupled to an external battery. Instead, the gating amplitude is solely governed by the position of the molecule relative to the conducting host (the ends of one-dimensional conducting host constitute the source and the drain of the transistor). This work has been published in two international journals. More work is in progress for devising a full understanding of AFTE and a paper is expected to result from that work.

\section{Publications}

Meunier, V., S. Kalinin, B. G. Sumpter. 2007. "Nonvolatile Memory Elements Based on the Intercalation of Organic Molecules Inside Carbon Nanotubes," Physical Review Letters 98, 056401.

Meunier, V. and B. G. Sumpter. 2007. "Tuning the Conductance of Carbon Nanotubes with Encapsulated Molecules," Nanotechnology 18, 424032. 


\title{
00406: Novel High-Resolution Micromechanical Gyroscope
}

\author{
Panos G. Datskos, Slobodan Rajic, and Nickolay V. Lavrik
}

\section{Project Description}

This proposal focuses on measurement of inertial forces using micro-electro-mechanical systems (MEMS). Our present approach for sensing angular acceleration uses a novel concept based on MEMS devices. Our approach is based on measuring the Coriolis force acting on an oscillating MEMS object while the MEMS object undergoes rotation. The present approach has the potential to far exceed this limitation, reaching (noise equivalent rotation) $N \boldsymbol{E} \Omega<\mathbf{1 0}^{-\mathbf{4}} \mathbf{d e g} / \mathbf{h}$ using much simpler structures especially, when multiple MEMS devices are fabricate in large arrays. During our present work we used finite-element analysis and fundamental theoretical models to design a series of mechanical structures with two degrees of freedom and various geometries. We fabricated MEMS gyroscope structures and conducted experimental studies of the gyroscope responses as a function of its rotational movements. In our experiments we used an optical detection technique (which we have developed previously) to measure resonance frequencies, amplitudes of oscillation, and deformations of MEMS devices.

\section{Mission Relevance}

The proposed project is relevant to DOE's Materials Science and Technology subprogram within the DOE Office of Science, national security mission, and nuclear security. The present work will advance the science of measurement of small forces and will impact many areas of science and sensors where measurement of small forces is required. Programs under way in the DOE's Office of Science will benefit from the knowledge gained during this work. The proposed work has relevance to the mission of other federal agencies. In particular DoD will benefit from advances made during this research as it applies to DoD navigation needs. Furthermore, the results of our studies provide an enabling component to develop a fyro-compass that does not rely on the earth's magnetic field but takes advantage of the earth's rotation. Our research is also relevant to the mission of Department of Homeland Security, where the need to obtain coordinates and navigation when the GPS capability is either denied or malfunctioning.

\section{Results and Accomplishments}

During FY 2007 we made significant progress in the advancement of measuring science, MEMS fabrication and materials. More specifically we made progress and accomplished the following:

(1) We modeled MEMS gyroscope structures using finite-element analysis utilizing materials that are compatible with microfabrication techniques. This allowed us to properly select materials and geometries that are possible to microfabricate while maintaining values for important parameters such as high resonance frequency (in the range of $\mathrm{kHz}$ ), high $Q$-factor on the order of 100,000, and reduced mechanical noise. 
(2) We designed new MEMS device geometries and topologies for the MEMS gyroscope structures that are compatible with existing microfabrication techniques without significantly reducing the expected performance characteristics. In order to achieve a high figure of merit (i.e., low noise equivalent $\Omega, N E \Omega_{\mathrm{z}}$ ) the device must have several parameters optimized. For example in order to minimize $N E \Omega_{\mathrm{z}}$, the resonator must have a low resonance frequency for the sensing element but high resonance frequency and driving amplitude for the driving resonator.

(3) We used bulk micromachining techniques to fabricate prototype MEMS devices that can operate as micromechanical gyroscopes. These devices will be tested, and using the models we developed we will refine the geometry and design which will be incorporated into the fabrication of future devices.

(4) We gained new theoretical understanding of the effect of small rotations of MEMS devices, especially at room temperature. The magnitude of the forces studied in this work is of the order of $10^{-16} \mathrm{~N}$. This is a relatively small force, and the effect of $k T$ has to be taken into account at temperatures above cryogenic. We used models that describe the resonator and take into account the noise due to thermal $k T$ motion.

The findings from our present work will greatly impact the area of science and sensors of measurement of small forces. The application of the knowledge gained during this work will enable the realization of MEMS gyroscopes that have figure of merit comparable to those of more expensive and larger gyroscopes.

\title{
00408: Demonstration of Intra-Reactor Diagnostics for Catalytic Fuel Reformers
}

\author{
Jae-Soon Choi, William P. Partridge, L. Curt Maxey, Johney B. Green, Galen B. Fisher
}

\section{Project Description}

Fuel reformers are a core enabling technology for advanced power-generation systems such as fuel-cell-based auxiliary power units. Fuel reformers are typically structured and catalyst-coated ceramic monoliths that convert hydrocarbon fuels into a hydrogen-rich gas stream via a complex network of reactions such as total and partial oxidation, steam and dry reforming, and water-gas shift. An enhanced fundamental understanding of these reactions, especially with respect to their spatial distributions and interactions, is required to develop and successfully implement reformer systems. However, in contrast with conventional packed-bed catalytic reactors, monolithic reformers have extreme spatial gradients in concentration and temperature. It is therefore difficult to extract process details such as the distribution of catalytic active sites and reaction mechanisms using a traditional laboratory approach that employs temporally and spatially integrated reactor-outlet measurements. In this project, we applied a new analytical approach to study reforming chemistry directly inside a working reformer. SpaciMS (Spatially Resolved Capillary Inlet Mass Spectrometry) and fine thermocouples or fiber-coupled thermometry were used to measure species and temperature distributions, respectively. The ultimate project goal 
was to demonstrate the ability of these minimally invasive diagnostics to measure useful information under realistic fuel reforming conditions. The proposed research tasks were (1) development of probes, probe-translation systems, and a reformer reactor; (2) assessment of undesired reactions inside the SpaciMS capillary probes; (3) evaluation of the probe's thermal and chemical stability; (4) identifying spatially resolved species and measuring temperature during catalytic methane and propane partial oxidation.

\section{Mission Relevance}

The compact fuel reformer is viewed as a critical technology for realizing national energy efficiency and environmental goals. This project, designed to help understand complex reformer chemistry and to build applied systems, directly supports the mission of various DOE programs. For example, fuel reformer research is an integral part of the Fossil Energy SECA (Solid State Energy Conversion Alliance) program. Moreover, Energy Efficiency and Renewable Energy's Office of FreedomCAR and Vehicle Technologies (OFCVT) can benefit from the proposed intra-reactor diagnostics in their research of high-efficiency, clean-combustion systems involving distributed hydrogen generation. The U.S. Department of Defense funds multiple research programs to develop advanced military power-generation technologies based on fuel cells. The results and capabilities acquired in this Seed Money project are already contributing to a DOE OFCVT program: the ORNL "Stretch Efficiency" project has just started to study fuel reformers as a means to increase the overall thermodynamic efficiency of internal combustion engine vehicles. Moreover, we have made significant progress to securing follow-on funding from SECA and are in the process of submitting a research proposal. This project has been the basis of expanding and establishing a solid relationship with the reformer technical and programmatic leaders within Delphi, a SECA industry partner and leader in the reformer area. In addition, we have established a collaborative relationship with U.S. Army Communications-Electronics Research, Development and Engineering Center and are exploring funding opportunities.

\section{Results and Accomplishments}

We have completed the four major project tasks outlined in Project Description section. First, probes, probe-translation systems, and reformer reactor capabilities were successfully developed and applied. Regarding intra-SpaciMS-probe reaction, we found that gas pools sampled under typical partial oxidation conditions do not suffer from any significant parasitic reactions inside the capillary probe, thus ensuring the quality of SpaciMS data in the study of intra-reformer chemistry. Moreover, the developed SpaciMS and temperature probes were robust and survived reforming conditions. Finally, we have conducted methane and propane partial oxidation at different space velocities, oxygen-to-carbon ratios, and thermal operational regimes and successfully measured local species and temperature profiles inside a Rh-containing honeycomb monolith reformer. These measurements provided new insights into the reforming process details. In particular, it was evidenced that neither of the two major mechanisms postulated in the literature for methane partial oxidation are correct; instead the actual reforming involves both mechanism (i.e., direct partial oxidation and sequential total oxidation-reforming). The propane study indicated an even higher process complexity involving three major axial reaction zones with various reactions in parallel and in sequence (e.g. total and partial oxidation, cracking, steam and dry reforming, water-gas shift). The ORNL-Delphi team used the intra-reactor data to refine an advanced reformer model. Even with these improvements, the model still did not 
follow the chemistry throughout the reformer; this demonstrates the limitation of models and model-based controls based on conventional measurement technology, and indicates the need for more advanced diagnostics like those demonstrated in this project. Nevertheless, the resultant measurement-modeling package led to enhanced understanding of the fuel-reforming process, and the project has demonstrated a viable process for further expanding this fundamental understanding of reformer chemistry. Such understanding will in turn help the development of efficient catalysts, reactor models, and control strategies. In summary, this project demonstrated the complexity of reformer function for even the simplest hydrocarbons, the need for intrareformer measurements to study more practically relevant liquid-fuel reformers, and the ability of the ORNL approach to meet the research challenges.

\title{
00409: Optical Monitoring of Delivery Methods for Therapeutic Agents to Neural Tissues
}

\author{
B. M. Evans, III, B. R. D’Urso, S. W. Allison, G. D. Griffin, and T. E. McKnight
}

\section{Project Description}

Portions of the brain may be either damaged or otherwise incapacitated due to stroke, tumor, or illness such as Parkinson's disease. A potential therapeutic advance for treating such conditions is the delivery of multipotent neural cells into damaged regions of the brain, but attempts to do so to date have met with only limited success. In fact, it has been shown that the majority of implanted cells do not survive 24 hours past the implantation procedure, and only 5-10\% of implanted cells survive a few weeks past the initial grafting procedure. It is unknown whether these cells survive the implantation process, or if they die subsequent to implanting procedures. Other research has shown that delivering precise quantities of cells to the implantation site is extremely important and that poor outcomes may result from either low or high cell densities. This research involves the investigation of fiber-optic based techniques for monitoring the delivery of cell-based therapeutics to the site of neural disease in order to determine the viability of cells as they are delivered. This research is part of a collaborative effort with researchers at the Virginia Commonwealth University School of Medicine and the University of Virginia and is part of a multi-institution effort to develop technology for harvesting small amounts healthy tissue from individuals affected by neural disease, growing healthy neural cells based on harvested tissues, and subsequently implanting these tissues in areas of the central nervous system that are affected by disease.

\section{Mission Relevance}

The goal of characterizing therapeutic materials delivered to neural systems simultaneously with the delivery of those materials is relevant to DOE's science mission. We are specifically addressing Science Strategic Goal General Goal 5, World-Class Scientific Research Capacity, and Program Goal 05.21.00.00, Harness the Power of Our Living World. These goals are similar to the medical applications and measurement sciences goals of the Office of Biological and Environmental Research. DOE has specific initiatives regarding interfacing with the neural 
environment in this office. The ability to monitor and categorize flows of materials is also of benefit to DOE's nuclear security mission.

\section{Results and Accomplishments}

During FY 2007, several primary objectives were completed. The primary objective accomplished was the design and fabrication of a prototype neural catheter with integrated fiber optical elements for performing cytometric measurments. This novel design incorporates the ability to measure optical intensity or attenuation, scattered energy, and fluorescence measurement. The initial prototype was less that $4 \mathrm{~mm}$ in diameter.

Vital staining techniques for enhancing cell detection were investigated. Also, an investigation of methods for tagging cells for detecting viability and apoptosis was performed. Apoptosis detection was performed using histological techniques for probing mitochondrial membrane potential. The mitochondrial membrane disruptor carbonyl cyanide 3-chlorophenylhydrazone (CCCP) was used to test the validity of the technique. Using these techniques we were able to detect viable and apoptotic cells using the cell monitoring catheter.

In vitro experiments with the cell monitoring catheter were performed in air and by infusing cells into a $0.6 \%$ agarose gel used as a brain-phantom material. GFP transfected 3RT1 cells and stained non-transfected RT2A rat glioma cells were used in these experiments. A stock solution of 3RT1 cells at a density of $1.2 \times 10^{6}$ per milliliter and of RT2A cells at $5.1 \times 10^{5}$ per milliliter was used for this work.

In summary, we have designed and tested a prototype cell-monitoring-device (CMD) that can be adapted to neurosurgical catheters. The CMD has cytometric capabilities compatible with those needed to confirm cell viability and quantity during cell delivery procedures. Photo-optical measurements of cell fluorescence made onboard this catheter during delivery of a cell suspension into a brain-phantom gel demonstrated the workability of the concept. The results suggest that the technique has sensitivity sufficient for accurate cytometric monitoring of cell delivery procedures, and future tests using an appropriate in vivo model will seek to confirm the clinical utility of the approach.

\section{Publications}

Evans, Boyd M., III, George T. Gillies, Stephen W. Allison; Helen L. Fillmore, Rachel L. Dyer, and William C. Broaddus. 2007. "Investigation of Neural Tissue Graft Delivery Using Instrumented Techniques." National Academies Keck Futures Initiative: Smart Prosthetics Exploring Assitive Devices for the Body and Mind, October 9-11, 2007. 


\title{
00423: Live Cell Micro-Arrays for Genotypic Evaluation of Microbial Electron Transport
}

\author{
M. Nance Ericson, Timothy E. McKnight, Michael E. Driscoll, Timothy Gardner, \\ and Michael L. Simpson
}

\section{Project Description}

Microbes capable of electron transport onto metals, also known as dissimilatory metal reducing bacteria (DMRB), have been exploited for applications in bioremediation and bioenergy. To date, the electron transport capabilities of these organisms have been analyzed using dissolved or solid metal substrates or fuel cell electrodes as electron acceptors in bulk microbial cultures. Such approaches are not suitable for real-time analysis of current flow, cell morphology or community structure. Moreover, the large volumes required by these approaches and the slow growth rate of DMRB species hinder the efficient analysis of the multitude of genetic and environmental factors influencing electron flux. To address these deficiencies, we are developing a novel platform for the real-time, single-cell, quantitative assay of metal reduction and current generation by DMRB. The platform consists of a fluidically coupled, transparent electrode array. This miniaturized approach will provide improved experimental control compared with traditional bulk culture techniques, enabling precise characterization of microbial electron transport as a function of cell number, cell morphology, community structure, surface composition and media environment. Techniques will be developed for controlled attachment on specific electrodes enabling simultaneous, live cell multistrain assays. The development of this technology will enable high-throughput analysis of DMRB physiology under precisely controlled growth and surface conditions, will enable quantitative modeling of microbial electron transport in dissimilatory metal reducing bacteria, and will accelerate optimization of bioremediative strategies and strain engineering for microbial fuel cells.

\section{Mission Relevance}

This research will form a foundation on which to further improve the science of microbial fuel cell design and microbe engineering for a number of application fields, including bioremediation, alternative fuels, and bioenergy. It has specific relevance to a number of DOE programs, including existing Office of Science's Biological and Environmental Research and Basic Energy Sciences objectives and new initiatives, including the DOE Bioenergy Research Center at ORNL. Furthermore, the eventual realization of practical microbial fuel cells for low-power applications such as remote sensing, implantable bioelectronics and flexible power for portable electronics will be of significant interest to other government entities including Defense Advanced Research Projects Agency, DoD, and National Aeronautics and Space Administration.

\section{Results and Accomplishments}

Microelectrode arrays were fabricated at the ORNL CNMS facility to facilitate microbial handling, growth, observation, and electron transport characterization. Each array incorporated 20 working electrodes composed of transparent indium tin oxide (ITO), enabling real-time visualization of microbial attachment and biofilm formation. Arrays were characterized with a variety of electrochemical assays prior to biological application to evaluate electrochemical 
performance and uniformity of electrode response. Electrode functionalization was also evaluated toward both improving electrode response in addition to providing future potential for controlled capture-and-release strategies of microbial cultures upon discrete electrodes. These strategies included both physical vapor deposition and electrodeposition of gold and electropolymerization of polypyrrole (pPy) and doped pPy films. Initial biological experiments were conducted with Shewanella oneidensis wild-type strain MR-1 under aerobic conditions, demonstrating uniform microbial adhesion and growth. Subsequent studies were performed under strict anaerobic conditions using both wild-type strain DSP10, and mutant strains, MtrA and MtrB, which are deficient in metal-reducing activity. Electrochemical characterization studies revealed several differences in electron transport activity of the various strains which may be indicative of these deficient metal-reducing pathways, thereby providing proof of principle of application of these platforms for screening these and related microbes for their electron transport characteristics. This platform and associated techniques, when fully realized, will provide new capabilities for improving the discovery and optimization of microbes for bioremediation, microbial fuel cells, and biofuel conversion processes.

\section{6: Plasma Etching and Simulation of Electron Scattering in Nanoscale Copper Interconnects to Minimize Size Effects}

Gary Alley, Nagraj Kulkarni, B. M. Evans, III, Hsin Wang, Harry Meyer, Bala Radhakrishnan, Don Nicholson, and Peter Todd

\section{Project Description}

The objective of this project is to research and development of a low-temperature plasma etching process for copper interconnects for feature sizes ranging from 25-1000 nm having controlled anisotropy, surface roughness, and etch rates. Diffusion, plasma etching, characterization, and process monitoring studies in copper films will provide guidelines for the control of plasma etching in patterned features. Furthermore, an electron scattering simulation that can model the experimental resistivity in such features as a function of various microstructural properties in copper films will be developed with input from existing first-principles, grain growth, and stochastic scattering models. Achievement of these objectives will provide for the first time a significant reduction of the size effect in the electrical resistivity of copper interconnects in semiconductor devices, which will meet the targets established by the International Technology Roadmap for Semiconductors for future generations of copper interconnects and enable the continuation of Moore's law in the foreseeable future.

\section{Mission Relevance}

If adopted by industry, this project may provide significant energy/cost savings and productivity gains for interconnect manufacturing, which accounts for more than half of all the chip manufacturing steps in the semiconductor industry. In addition, significant environmental benefits due to minimization of process steps such as copper chemical mechanical planarization and elimination of copper electroplating are expected. The modeling thrust in this project will investigate the scientific reasons for electron scattering in narrow interconnects that causes the 
size effect phenomenon in copper interconnects. These include factors such as the grain size and distribution, texture, geometry, and interfacial properties. Possible DOE programs benefiting from this research are programs in Energy Efficiency and Renewable Energy (EERE) on nanomanufacturing, nanotechnology, and power electronics packaging.

\title{
Results and Accomplishments
}

The plasma etch tool that is central to the seed project was not operational for the experimental work in FY 2007. An upgrade to install an electrostatic chuck permitting circulation of a lowtemperature coolant (up to $-80^{\circ} \mathrm{C}$ with a chiller) is currently under way. Meanwhile, we have made progress in preliminary modeling of some special grain boundary $(\mathrm{gb})$ resistivities in copper such as $\Sigma 3$ twin boundaries and $\Sigma 5$ twist boundaries. The $\Sigma 5$ resistivity $\left(31 \times 10^{-17} \mathrm{ohm}\right.$ $\mathrm{m}^{2}$ ) was found to be an order of magnitude larger than that of $\Sigma 3\left(1.1 \times 10^{-17} \mathrm{ohm}-\mathrm{m}^{2}\right)$, which confirms measurements in the literature. The more general case of low- and high-angle boundaries will be the subject of future work. For mesoscale microstructure simulation, a Monte Carlo technique was used to simulate the evolution of grain structure when the growth dimension was constrained. The simulations also took into account diffusion along the free surface of a thin film. All grain boundaries were assumed to be of the high-angle type with identical energies and mobilities. Surface diffusion led to the formation of a grain boundary groove. Various geometries involving confinement in one, two, and all three dimensions were studied in order to understand the influence of the constraint on grain morphology. The results indicated that in all cases the grain growth ceased after the mean grain size was roughly equal to the film thickness. A bamboo structure was obtained in all cases, and there was no evidence for the stagnation of an equiaxed grain structure for any of the geometries studied.

\section{7: Electronic Colorimetric Monitor}

\author{
R. J. Warmack, C. L. Britton, and J. E. Hardy
}

\section{Project Description}

A demonstration sensor will be designed and tested to demonstrate the validity of using a miniature electro-optical readout for colorimetric sensing of toxic vapors and chemical warfare agents. Although most colorimetric materials are read visually, there are many situations that require automatic determination of hazardous vapors. For example, a badge device could automatically and immediately alert the wearer of significant exposure before an overdose can occur. In another example, protective canister filters can become fully loaded and allow chemical vapor breakthrough to the user. A sensor positioned inside the filter could indicate that the filter is being consumed and provide advance warning before the filter is fully consumed.

This short-term project will develop a matchbox-sized reader that could eventually be reduced in size to less than few cubic millimeters on each side. The device is based upon well-proven colorimetric indicator material but incorporates a novel miniature optical reader to convert optical changes into electrical readout. Such a miniature system is unavailable commercially and is a first step toward realizing a microminiaturized dosimeter for toxic vapors. Calculations show 
that the desired limits-of-detection can be achieved both with the demonstration module and with the ultimate package consisting of tiny light-emitting diodes (LED) chips, photodiode, and readout chip in a few $\mathrm{mm}^{3}$ package. The proposed demonstration will allow ORNL to begin a program to develop an integrated package that is applicable for electronically reading a broad range of colorimetric materials.

\section{Mission Relevance}

Measurement science and technology is essential for monitoring and controlling processes to achieve better energy efficiency or to generate fewer waste products. Both of these outcomes are important parameters to DOE's mission and numerous programs including the Science-to-Energy agenda. Applications for low-cost, low-power microsensors and preconcentrators include buildings, transportation, and energy conversion. The demonstration of an automatic hazardous vapor sensor and that has the potential to rival laboratory analytical instrumentation should prove very attractive to DOE to allow sensors for environmental, industrial, and personnel monitoring where size, power, and real-time detection of low levels of analytes are important. The implications for homeland security and the military are obvious for a toxic-agent monitor that can be reliably produced at low cost in high-volume quantities.

\section{Results and Accomplishments}

Two miniature prototypes were designed, fabricated, and tested. Optical calculations were performed to determine optimum placement of the LEDs, photodetectors (PDs), and colorimetric material. Experiments confirmed the calculations of signal amplitude and noise. A reference channel was added to ensure that variations in voltage of LED intensity would not cause false readings. Tests were performed as a function of dose to toxic vapors using commercial colorimetric paper. The tests established that a postage-stamp-sized device could provide adequate sensitivity and stability. Furthermore, the possibility of a microminiaturized package using LED, PD and application-specific integrated circuit (ASIC) die should be a straightforward manufacturing exercise. Already, a microfabricated silicon microphone and an ASIC die are integrated into package of $4 \times 5 \times 1 \mathrm{~mm}^{3}$ and are produced for cell phones for less than $\$ 0.50$ each. This next miniaturization step for the colorimetric monitor is anticipated for a variety of personnel protection applications in which a disposable automatic toxic-vapor monitor is desirable. 


\section{3: A Compact Chemical-to-Hydraulic Power Source}

Lonnie J. Love

\section{Project Description}

A strong need exists in the robotic community for a compact power source ( 30 to $400 \mathrm{ci}$ in volume) that converts chemical energy into high-pressure $(>1,000 \mathrm{psi})$ hydraulic energy. The overall noise, gas, and thermal emission should allow human beings to work in close proximity to it. Applications for such a power source would range from untethered mobile robotics, prosthetic legs, to small portable power sources for emergency and military workers (i.e., to operate hydraulic tools). There is an additional constraint imposed upon the power source based on the nature of these applications in that the load demand is highly varying in magnitude (up to $50 \%$ of the average) and over a relatively short time duration $(\sim 1 \mathrm{sec})$. This additional constraint severely limits the current performance of most conventional power sources such as batteries. Finally, the energy source has to be in a form that is directly useable for mechanical work. Miniature hydraulic actuators are ideal for robotic-like applications.

\section{Mission Relevance}

Untethered mobile robotics will play a significant role in future remote dismantling and decontamination (D\&D) work for DOE, including work on the ORNL reservation. Power supplies that are human friendly and can supply significantly more energy than conventional batteries are necessary to perform D\&D types of tasks. In addition, the military, and in particular the Defense Advanced Research Projects Agency, always has an interest in compact power sources. Furthermore, there is also a potential new program in FY 2008 for the Office of Naval Research and the Navy in the area of automated weapon assembly, magazine packup and unpack, and ordnance breakup (out of containers) — all in the area of high payload manipulation and all in the magazine area of the ship, which would make the power source being proposed very attractive.

\section{Results and Accomplishments}

This research is to determine the feasibility of a compact, human-friendly power source that significantly exceeds current battery capabilities, and it is mainly an exploratory study. The main path that was explored was a chemical reaction such as one that produces a hydrogen gas that then undergoes a combustion process and then is converted into hydraulic energy. We completed a very detailed chemical search that verified that sodium borohydride reactions are the optimal for such a power supply in terms of overall power density. The catalytic generation of hydrogen gas was found to be the major technical hurdle. To solve this problem, a number of centrifugal hydrogen reactors (gas separators) were designed and tested. Finally the feasibility of utilizing a compact catalytic combustor to burn the hydrogen gas to power a turbine was tested and proven. 


\title{
00356: Alzheimer's Disease Detection via Nonlinear Analysis of EEG
}

\author{
Nancy B. Munro and Lee M. Hively
}

\section{Project Description}

We wish to enable presymptomatic diagnosis and early treatment of Alzheimer's disease (AD). Thus, we propose analysis of human scalp electroencephalogram (EEG) data by ORNL's novel phase-space dissimilarity measures (PSDM), coupled with novel applications of analysis of variability in an attribute of the discrete distribution function and also statistical network characterization. The goal is determination of dynamic signatures to distinguish among the following four groups of aged patients: (1) normal; (2) mild cognitive impairment (MCI); (3) early AD; and (4) dementia with diffuse Lewy body disease (DLB). This project is being conducted in collaboration with the University of Kentucky (UK), Chandler College of Medicine's Sanders-Brown Center on Aging/Alzheimer's Disease Research Center and Department of Behavioral Science. The goal of this project is to show proof of concept for early detection of Alzheimer's disease and possibly even the pre-Alzheimer's changes evidenced in MCI patients as well as early detection of DLB. The earlier cognitive decline can be detected and treatment started, the more effective the treatment is in slowing brain deterioration. The data obtained in this project will serve as preliminary data for an National Institutes of Health proposal for follow-on funding.

\section{Mission Relevance}

The technology developed under this project will be novel, general, patentable, and applicable to other biomedical endpoints and also to predictive maintenance of machines using electrical power, including critical infrastructure in nuclear power plants and other energy-production facilities. The technology, if successful, will open up lines of related research on neurodegenerative diseases, other dynamic brain disorders, susceptibility to addiction, and a variety of additional biomedical diagnostic applications (e.g., brain biometric and voice signature analyses). Success in this project will be of high interest to the NIH's Institute of Aging (NIA) according to Dr. Susan Molchan of the NIA, both as a biomarker for early Alzheimer's diagnosis and also in the context of the NIA program on biomarkers of aging. Success is also of interest to NIH's Institute of Bioimaging and Bioengineering (NIBIB), according to Dr. Grace Peng of that institute.

\section{Results and Accomplishments}

In FY 2007, work continued on mathematical methods development for data analysis using surrogate EEG data. The UK furnished an additional 14 training data sets for a total of 28 usable sets plus an unknown number of test data sets held by UK. The present data are not yet adequate for obtaining statistically significant results; full-scale analysis by ORNL's methods will proceed when 10 training data sets for each of the experimental groups are obtained. Analysis to date has focused on data quality analysis and developing and testing the research-class analysis software. Collaborators have furnished their qEEG algorithm and code to ORNL for use in analyzing the 
EEG data from the delayed recall task portion of the protocol. A student has been identified who will do data analysis at no cost to the project once methods are ready for implementation. No publications have resulted to date from this project but will be prepared when results are obtained. One presentation was given in June 2006.

\title{
00411: Determining Relative Value of Ecosystem Services
}

\author{
Rebecca Efroymson, Henriette Jager, and Gbadebo Oladosu
}

\section{Project Description}

Ecosystem valuation is the combination of methods by which dollar values or other value metrics are assigned to components of the environment, such as wildlife populations, individual animals, and forest communities, as well as processes termed "ecosystem functions" or "services" such as decomposition, pollination, purification of water, provision of habitat, photosynthesis, nitrogen fixation, and carbon sequestration. Existing methods for valuing ecological functions or services fail to value related functions or services consistently or completely. Most applications are based on surveys of human preferences. We developed a proof-of-principle methodology for analyzing the concept that dollar values (or other metrics) of ecological entities that are established through markets or surveys can be extended to other ecological entities through models of ecological relationships. Our work addressed several scientific problems:

- How can ecosystem valuation, which reflects human preferences, also be anchored in ecological relationships?

- How can economic models incorporate simple and complex ecological relationships that control supplies of raw materials?

- What is the most effective case study or studies to demonstrate the incorporation of ecological algorithms in valuation of ecological services?

To answer these questions, our project (1) reviewed the existing use of ecological relationships in relative ecological valuation, (2) illustrated how these relationships and more complex models could be used in relative valuation, and (3) described and demonstrated potential applications of relative ecological valuation.

\section{Mission Relevance}

Various federal agencies and utilities (EPA, DoD, DOE, USDA, NOAA, Electric Power Research Institute) are converging on the need to develop reliable and rigorous methods for valuation of ecological populations, communities, functions, and scenarios involving ecological change. Examples of potential applications of this approach include the selection of contaminant remediation alternatives, the evaluation of environmental benefits of research programs, the quantification of appropriate damages to compensate for natural resource injuries, the valuation of natural disaster mitigation services of ecosystems, and the quantification of appropriate incentives for conservation. For example, the DOE Office of Legacy Management might benefit from this research, because methods developed here may be used to prioritize long-term 
stewardship needs. Offices of DoD have expressed interest in the development of environmental benefit indicators to quantify ecological services of the natural resources of military installations.

\section{Results and Accomplishments}

We identified classes of ecologically based benefits transfers that have previously been used in ecosystem valuation: predator to prey, ecosystem to ecosystem, organism to habitat, commodity to ecosystem service provider, and ecosystem service at one site to ecosystem service at another. After determining limitations of these transfers, we demonstrated how additional ecological concepts and linkages may be added to ecosystem valuation. These include spatial arrangement of habitat and thresholds in ecosystem services. We demonstrated that habitat valuation methods and reserve selection models could be modified to include adjustments for distance-based measures of habitat suitability, edge-based value, habitat connectivity, habitat patch size thresholds, and the spatial relationship between complementary ecological services. We showed that measures of the value of ecological structure and function might be transferred in either direction. These findings were presented to an interagency group, including representatives of the Army Corps of Engineers, EPA, and USDA. Next, we developed theoretical relationships between marginal use and nonuse values assigned to rare wildlife populations. We quantified this relationship for a case study involving a population viability analysis model to predict Chinook salmon population size and a relationship between willingness to pay and salmon population size derived from a meta-analysis of survey data conducted by J. Loomis of Colorado State University. Results showed that the marginal value of salmon peaked at an intermediate population size, whereas total value continued to increase.

Ecosystem valuation can be improved by incorporating measured and/or simulated ecological relationships if we understand how total or marginal value varies with parameters that can be measured or modeled.

\section{3: Effects of Groundwater Chemistry on the Distribution of Soil Microorganisms in Natural Media}

Philip M. Jardine, Tracy L. Bank, and Matthew Fields

\section{Project Description}

Our research investigated the influence of groundwater chemistry on the distribution of bacterial communities between the aqueous and solid phases in natural sediments using a novel, bench-top study. These investigations used contaminated and non-contaminated intact sediment samples from the Field Research Center (FRC) at ORNL. Previous studies have suggested that changing groundwater chemistry, which resulted from legacy waste burial, leads to changes in the microbial communities present in contaminated versus uncontaminated groundwater at the FRC. We characterized the microbial communities present in aqueous- and solid-phase sediment samples from three areas of the FRC with similar geology but vastly different groundwater chemistry. We simulated changing groundwater conditions and determined if the distribution of bacteria between the solid and aqueous phases changed using intact sediment column 
experiments. We used atomic force microscopy (AFM) to attempt the measurement of the nanoscale forces of adhesion between the aqueous-phase bacteria and mineral phases present in the sediments. Our experiments will determine if bacterial stickiness to mineral surfaces can explain the distribution of cells between the aqueous and solid phases in natural sediment.

\section{Mission Relevance}

The project is directly relevant to DOE's environmental quality and science missions. Our project uses nanotechnology and molecular biology tools to characterize complex microbial communities in natural soils for environmental use. Our project provides an innovative application of AFM that will be of interest to a wide range of scientists involved in nanoscale research. If successful, we expect that this project will lead to broader applications within the subsurface biogeochemistry and microbial ecology elements of the Environmental Remediation Science program and the Genomes to Life program. In our study we introduce a new and controversial approach to studying biodiversity using geochemical tactics rather than a classical microbial approach which may provide new insight into microbial diversity at the FRC and related contaminated sites. Results from our study may also be relevant to studies of bacterial and colloidal transport in porous media.

\section{Results and Accomplishments}

Intact sediment cores were collected using Geoprobe technology from three areas within the FRC which corresponded to low-, medium-, and high-ionic strength subsurface environments. Samples were stored anaerobic and aseptic until use in miscible displacement experiments which involved packing the various sediments into glass columns, purging with $\mathrm{CO}_{2}$, and leaching the soils with solutions of similar ionic strength and solute composition as that observed in situ. Effluent was collected as a function of time and analyzed for microbial biomass and bacterial adhesion using AFM. Column effluent showed significant differences in bacterial counts that were dependent on ionic strength. High-ionic-strength influent washed off significantly less bacteria compared to the low-ionic-strength influent, suggesting that repulsive forces between bacteria and mineral surfaces are increased when solution ionic strength decreases. Bacterial adhesion measurements are pending as numerous earlier attempts have failed to isolate bacteria on AFM tips in order to acquire force data. Microbial community analyses are also being completed on the solid and aqueous samples, and preliminary results suggest that nucleic acid yields were low and indicative of low bacterial numbers, as previously observed. Diverse microbial communities were present, however, and consisted of $\beta$-Proteobacteria classified as Curvibacter and Delftia species. Isolates of these genera are typically nitrate reducers and are commonly observed in groundwater and sediments. A second molecular technique was also used that indicated the presence of two different major microbial populations. The different primer sets indicated different population structure and composition, suggesting that the use of multiple molecular strategies might provide an improved sampling of bacterial diversity in subsurface environments. Additional clone sequences are being attempted. Coupling these results with pending AFM experiments will assist with whether bacterial stickiness to mineral surfaces can explain the distribution of cells between the aqueous and solid phases in natural sediment. 


\title{
00419: Multivariate Statistical Analysis Technique to Locate Ecological Observation Sites within Regional Landscapes
}

\author{
Patrick Mulholland, William Hargrove, and Latha Baskaran
}

\section{Project Description}

The objective of this seed money project was to test a multivariate statistical technique that will produce landscape maps of biophysical similarity for regional assessment. Specifically, we evaluated the application of a multivariate geographic clustering approach to locate observation sites in the National Ecological Observatory Network (NEON) domain centered on East Tennessee (Southern Appalachians/Cumberland Plateau region) and demonstrate the robust nature of this approach for site selection for regional and continental-scale landscape analysis and monitoring. We conducted three types of within-domain representativeness analyses based on groups of input map layers representing (1) soil variables, (2) vegetation variables, and (3) ecosystem process variables. These techniques were used to aid site selection for a response to a request for information to be issued by the National Science Foundation (NSF) for the NEON program (www.neoninc.org). The new analyses we conducted helped ORNL identify regionally representative observation sites that satisfied the NEON goals. The information generated strengthened our design prospectus for NEON measurement sites within the Appalachian/Cumberland Domain that included East Tennessee. The regional analysis methods should also prove to be valuable for other programmatic site selection activities.

\section{Mission Relevance}

This project benefited the DOE mission of monitoring and improving environmental quality. The return on investment for this work will be twofold: (1) by demonstrating the usefulness of multivariate geographic clustering, we can provide a defensible method for selecting representative sites in NEON and in a variety of other agency programs, and (2) by developing a more detailed understanding of our own NEON domain, we will maximize the likelihood that an ORNL-led design prospectus is selected by NEON for construction of NEON measurement sites within our regional domain. If selected, the construction of substantial NEON infrastructure for ecological measurements in the eastern Tennessee region, paid for by the NSF, will provide substantial leveraging opportunities for ORNL and DOE and will benefit the Life Sciences Directorate. In addition to the immediate value to DOE and ORNL, this work also will benefit the NSF as it designs and constructs new national ecological observatories. The project may also benefit other federal agencies that have monitoring networks, like U.S. Department of the Interior National Park Service, and may help the U.S. Department of Agriculture to find locations appropriate for growing new crop varieties and to ensure that representative germplasm have been stockpiled and preserved.

\section{Results and Accomplishments}

A combination of multivariate analysis of nine climatic variables and ecological expertise was initially used to create 20 NEON Domains. Each climatic variable is itself a map at $1 \mathrm{~km}^{2}$ resolution over the conterminous United States, consisting of nearly 8 million cells. The same multivariate tools that were used to help create the 20 NEON Domains were then used to develop 
"representativeness" maps for each regional domain based on other variables (variables other than climate characteristics). Separate representativeness maps were developed based on gross primary productivity and respiration data, soil and topography data, and vegetation characteristics data (http://research.esd.ornl.gov/ hnw/neon/withindomainrep2/). These maps were then used in subsequent analyses to determine regional variability in ecological characteristics and to aid in the potential deployment of instrumentation across ecological gradients. The maps represent nonclimatic gradients in several key ecological characteristics grouped together and thus differ from the single-variable mapping that is commonly used to identify ecological gradients. This grouping of several ecological characteristics into a rigorous and quantitative digital database offers a more sophisticated basis on which to make decisions on locations for ecological sampling and analysis.

We were notified in April 2007 that the Oak Ridge Reservation, specifically Walker Branch Watershed, was tentatively selected as a core wildland NEON site. Final decisions on NEON site selection will be made in early 2008 .

\section{9: Development of an Advanced Surface-Enhanced Raman Spectroscopy for the Identification and Characterization of Pollen}

Shannon Mahurin, Meng-Dawn Cheng, and David Gossage

\section{Project Description}

The purpose of this project is to develop an advanced detection system for the identification and characterization of airborne, allergy-significant pollen utilizing the surface-enhanced Raman spectroscopy (SERS) technique. Raman spectroscopy provides chemical information through excitation of characteristic molecular vibrations. Raman signals of pollen are enhanced by attaching silver nanoparticles to the grains under flow conditions. This permits continuous in situ sampling and identification of the pollen grains as they flow through the system. resulting in a significant reduction in identification time as well as an increase in throughput. In addition to pollen identification, the chemical information provided by Raman spectroscopy allows us to characterize the chemical composition of the pollen grain and detect surface species attached to the grain. The effect of pollutants such as nitrogen dioxide on the pollen grain and attached surface species is explored using the SERS technique.

\section{Mission Relevance}

This project is relevant to the DOE mission of environmental quality through the reduction of the impact of energy production on the environment. Since the interaction of pollen with environmental pollutants has been suggested as one potential cause of the recent increase in allergy-related diseases in children, a reduction in pollutant concentration could reduce the number of respiratory problems in children and, potentially, the general population. This project also has significance to the Department of Homeland Security since the SERS technique can be used as a method for the identification and discrimination of pathogenic vs. nonpathogenic bioaerosols, an area that has received focus primarily due to the recent national concern over 
bioterrorism. This project has significant potential to benefit other federal agencies such as the National Institute of Allergy and Infectious Diseases at the National Institutes of Health (NIH), whose mission is to better understand immunological and allergic diseases. The development of a technique to improve the characterization of a respiratory irritant such as pollen and to better understand the effect of environmental pollutants on the composition of pollen is ideally suited to the mission of NIH.

\section{Results and Accomplishments}

During FY 2007, a microscope-based Raman system was constructed and evaluated to allow for simultaneous imaging and Raman spectroscopic measurements of pollen grains, with some capability of Raman imaging. We measured Raman spectra for three different pollen sampleswhite pine, ragweed, and tag alder-using the Raman instrument and obtained a unique Raman signature for each pollen type, effectively verifying the application of Raman spectroscopy to the identification of pollen. Silver nanoparticles were successfully attached to the pollen; however, SERS enhancements factors have thus far been small. The low enhancement factors are possibly due to weak silver-pollen interactions that result from surface charges on the pollen. Rhodamine 6g (R6g), a negatively charged organic molecule used as a Raman calibration standard, exhibited a strong SERS signal when attached to only the silver nanoparticles but a weak enhancement when combined with pollen and then with the silver nanoparticles. Attempts to improve the silver-pollen interaction through modification of the surface charge on the pollen have produced limited gains in the SERS enhancement factors. We are currently working to optimize SERS signals and measure spectra from surface species extracted from the pollen grains. Finally, we exposed three different pollen grains to an atmosphere containing nitrogen dioxide at a $5 \mathrm{ppm}$ concentration and observed no change in the Raman spectrum, indicating that much of the Raman signal originates from the outer wall of the pollen which is chemically very stable. We are continuing to collaborate with Greer Laboratories to measure the effect of pollution on pollen focusing on changes in the surface species (e.g., antigens) located on the pollen outer wall.

\section{Publications}

Mukherjee, D. and M. Cheng. 2008. "Quantitative Analysis of Carbonaceous Aerosols Using Laser-Induced Breakdown Spectroscopy: A Study on Mass Loading Induced Plasma Matrix Effects." JAAS. 23, 119-128. 


\title{
00430: Land-Use Dynamics and Infectious Diseases: A Systems Approach to Defining the Causal Mechanism of Outbreak and Spread of Eastern Equine Encephalomyelitis
}

\author{
Virginia Dale, Charles T. Garten, Amy Wolfe, Latha Baskaran
}

\section{Project Description}

Insect-borne diseases affect millions of people globally, yet there are large gaps in knowledge about the conditions under which such diseases are likely to emerge and proliferate One insectborne disease, eastern equine encephalitis (EEE), is caused by the EEE virus (EEEv), which is transmitted by mosquitoes throughout the Americas. Under this project, we were able to build a collaborative partnership among landscape ecologists at ORNL, medical professionals at Vanderbilt University and the Tennessee Department of Health, and hydrologists at Georgia Tech. Our new team demonstrated that EEE epitomizes a coupled system that cannot be understood by investigating only the component parts. We showed how changing patterns of mosquito-borne disease exposure risk can be understood by delineating and quantifying the interrelationships among coupled social, biological, and physical systems. To help us understand this system, we developed a model of the coupled systems: host and vector biology, social aspects of land use and human behavior, landscape ecology (including patterns, composition and structure of land cover and land-cover change), hydrology (both surface wetness and wetland depth) and climate (temperature and precipitation). We also used a retrospective analysis of the occurrence of EEE in the southeastern United States to demonstrate the importance of these relationships to explain patterns of disease occurrence.

\section{Mission Relevance}

EEEv is the most virulent mosquito-borne virus in the world, causing severe disease in humans and equines, often leading to death within a very short period of time. The mortality rate in humans and horses ranges from 35 to $90 \%$. EEEv also is a potential bioterrorist agent because of its ability to be weaponized. In North America, EEEv is mostly seen along the Atlantic and Gulf coasts, typically in wetland habitats. These same wetland ecosystems are valued for subsistence, and recreation activities, their appeal to nearby homeowners, water quality, flood control, and biodiversity. Moreover, wetlands are valued as a national resource and are protected and encouraged by both state and federal legislation. Our new conceptual model and retrospective analysis captures how fundamental understanding is critical for identifying successful ways to influence the spread of EEE. While our project focused on improving fundamental understanding of EEE, our new way of looking at the disease system will help us develop research plans appropriate for the National Science Foundation and, possibly for the National Institutes of Heath and the Department of Defense.

\section{Results and Accomplishments}

Our team completed two tasks. First we developed a prototype interdisciplinary model of how EEE occurs and spreads within broad spatial environments. This model encapsulates our understanding of how environmental factors contribute to emergence and transmission of EEE in the southeastern United States. Feedback addressed by the EEE model includes human behavior, 
landscape change, host abundance, vector breeding, and risk of infection. For example, human behavior can affect land use by the presence of people near wetlands. Land-use pressures can create, change, or destroy wetlands and, thereby, alter the landscape that provides the breeding conditions necessary for the EEE infection. In addition, human behavior also situates humans within the EEE system even when people choose to limit their activities in or near wetlands to reduce their risk of exposure to EEE. The key components of our coupled EEE model-human behavior, landscape change, vector breeding, and risk of infection-operate at different spatial and temporal scales. For example, the effects of wetness on habitat can change on time scales of minutes or hours; whereas, effects of land use can operate on multiyear or multidecade scales. We developed the coupled model to ensure that components operating at different spatial or temporal scales interface smoothly.

Our second task was to use a retrospective analysis to assess and validate our understanding of factors affecting EEE occurrence in Tennessee and Georgia. The retrospective study allowed exploration of relationships among EEE host species diversity, land cover diversity, and changing land cover patterns and opportunities, and other pressures that affect wetland conditions. We demonstrated how increased risk of EEE transmission and outbreak relates to breeding vectors, land-use change, and human behavior. We documented that the mosquito species of most importance is different in coastal and inland states. In coastal states, the mosquito Culiseta melanura plays the key role in disease transmission; whereas, Culex (Melanoconion) erraticus is the dominant vector among birds in inland states. Cx. erraticus is less capable of transmitting EEEv than Cs. meanura even though it is a less efficient vector and infected specimens are found less frequently than Cs. melanura. Humans, beyond serving as bridge vectors, also interact with swamps and change swamp ecology. For example, deforestation of cypress trees in the early 1900s probably made inland swamps less habitable for Cs. melanura and more habitable for $C x$. erraticus. Changes in landscape and human behavior that bring them in proximity to these lands (e.g., via duck hunting or participating in other activities close to marshes) ultimately impact EEEv transmission to humans.

\title{
00434: A Proof-of-Concept Implementation for a USA National Phenology Network CyberInfrastructure
}

\author{
Bruce E. Wilson, Giri Palanisamy, and Benjamin C. Crom
}

\section{Project Description}

Phenology is the study of periodic plant and animal life cycle events, particularly those driven by changes in climate. Phenological measurements are extremely valuable indicators of local, regional, and global climate changes. Further, phenological variations have crucial environmental and socio-economic implications for health, agriculture, and natural resource management. Despite this importance, broadly distributed phenological measurements are almost nonexistent in the USA. Over the past two years, substantial momentum has developed for a USA National Phenology Network (USA-NPN), with activities such as workshops funded by several federal agencies. Given ORNL's existing expertise in environmental and biological 
informatics and our participation in USA-NPN planning activities, we have a leading position as a possible institution to be the cyberinfrastructure host for the proposed USA-NPN. The objective of this project is to maintain and enhance that position by developing proof-of-concept implementations of key informatics technologies which are needed for USA-NPN.

\section{Mission Relevance}

There are two areas of benefit to DOE. The first area is that phenological measurements are important to climate change research, particularly in terms of ground truth data for measuring the effects of climate change. Within this area, phenological measurements are particularly useful as inputs and validation measurements for climate model research funded by DOE. The second area is that the informatics technologies that we will be developing in this project should be of use to other ecological research areas. It is possible, though far from certain, that these informatics technologies will have applicability to other data and information management needs within DOE.

\section{Results and Accomplishments}

We developed a new data model for phenological observational data, based on existing data and on input from a workshop held in Tucson, AZ in May 2007. We have tested this data model against a range of historical phenological observational data and made modest changes to accommodate the results of that testing.

We completely rewrote the user registration and user data entry pages for the plant phenology network, which is a participating network in USA-NPN. This work greatly reduces the amount of administrative effort needed to provision and manage observers in USA-NPN participating networks.

We developed a prototype metadata entry, harvesting, indexing, and searching tool for phenological data, based on the ORNL Mercury technology. This tool was not deployed in FY 2007, but we anticipate that it will be deployed in early FY 2008.

We completed the design of the information architecture to underlie the new USA-NPN informational and data collection web site. This information architecture involves identifying the various user groups for the site and determining what information is of greatest interest to those user groups. This information architecture is being used in FY 2008 for the implementation of the new USA-NPN web site.

Based on our involvement with this work, Bruce Wilson was selected to be a part of the USANPN Board of Directors, for a two-year term beginning in November 2007.

\section{Publications}

Betancourt, J., et al. 2007. "Evolving Plans for a USA-National Phenology Network (USANPN)." Eos Trans. AGU. 88, 211-211. 


\title{
00441: Quantitative Parametric Decay Simulation: A New Tool for Understanding Parasitic Nonlinear RF Power Losses in Heating Fusion Plasmas
}

\author{
L. A. Berry and E. F. Jaeger
}

\section{Project Description}

The propagation of radio frequency (RF) waves in a plasma is typically described by linear theory where the amplitude of the wave in the plasma is proportional to the strength of the source. However, many experiments see the generation of new waves at the plasma edge as the amplitude of the source is increased. These new waves increase rapidly with source power and can heat the plasma edge and reduce plasma performance. These losses are a critical issue for experiments like ITER where the edge power loss can lead to undesired component heating as well as to reduced power for producing fusion power in the core. Based on experimental data and qualitative models, these new waves are generated by parametric decay instabilities (PDI). This qualitative theory is not able to predict the quantitative losses as plasma and RF heating parameters are varied. This project, through a combination of analytic and computer-based simulations, will develop a 1D quantitative model of the parasitic power loss due to PDIs. This will provide new understanding of PDI physics as well as suggest possible approaches to controlling the power loss.

\section{Mission Relevance}

The project is relevant to both the DOE science and energy missions. The most direct application of the research is to programs in the Office of Fusion Energy Science where radio-frequency power is widely used for heating magnetically confined plasmas, including ITER. The project is also relevant to basic plasma research efforts in the Office of Science.

\section{Results and Accomplishments}

(1) A model for PDI was formulated for the 1D All Orders Spectral Algorithm (AORSA1D). Energy conservation relations were then derived from this model to provide a check on the numerical results. (2) AORSA1D was modified to obtain solutions to this model. This effort comprised creating 1D solutions for each of the three PDI waves, coupling them with the nonlinear terms from the aforementioned model, and solving using iterative techniques with overrelaxation. (3) PDI runs for the National Spherical Tokamak Experiment (NSTX) at Princeton, the Alcator C-Mod experiment at MIT, and the international ITER facility to be built in France were analyzed. (4) The results from these analyses have increased our understanding of PDI issues. First, the AORSA PDI model includes only convective mechanisms (i.e., power is carried away from the instability zone by the waves) for the saturation of instability. While there may be numerical analysis issues, we observed unlimited growth (numerical instability) at power levels below that seen in experiments, but in rough agreement with analytic estimates. Either improved numerics or additional nonlinear power absorption mechanisms are required. Second, 
the NSTX results, with multiple zones of possible instability, are different and do not agree with even the analytic estimates, suggesting that our assumption of local instability may not be correct. Finally, estimates of the PDI impact on ITER suggest that there should be no significant impact on operational range. 


\section{1: Diamond Films on Low-Cost Substrates for Wide-Ranging Electronic Applications}

Lee Heatherly, Leslie Wilson, Robert W. Shaw, and Amit Goyal

\section{Project Description}

The superlative properties of diamond make it an attractive candidate for high-performance electronic devices. Diamond has an extremely high dielectric constant, high thermal conductance, high carrier mobility, and a negative electron affinity. These properties make it an ideal material for the manufacture of high-temperature semiconductors, ultra-fast semiconductors, high-voltage semiconductors, and cold cathode electron emitters for flat-panel displays and field emission vacuum diodes. For most of these applications, the diamond must be in the form of near-single or single crystal geometry. This project deals with a procedure that can possibly provide large-area, single-crystal diamond thin films suitable for many of the applications mentioned above. The procedure involves (1) the development of a suitable textured substrate that includes a thin iridium film as the top surface, (2) the dense nucleation of oriented diamond seed crystals on the iridium, (3) the growth of an epitaxial diamond film from the seed crystals, and (4) the characterization of the resulting diamond film as well as each step of the process.

\section{Mission Relevance}

This work is relevant to several possible federal as well as private funding sources. The Office of Electrical Transmission and Distribution program is expected to receive funding for an initiative on high-power electronics, and diamond films are of great interest. The Defense Advanced Research Projects Agency (DARPA) is likely to be extremely interested in diamond-based devices. The National Reconnaissance Office is funding work on the development of solar cells using polycrystalline diamond films for space applications, and this work should be very relevant to this effort. NASA is likely to be very interested in these films for photovoltaic applications in space. As for private sources, the semiconductor industry in general will be very interested in a technology that promises mass production of multi-chip modules and faster devices.

\section{Results and Accomplishments}

During FY 2006-07, several of the stated goals were accomplished. (1) A suitable buffer stack was developed for the Ni-W RABiTS that allowed iridium and diamond to be epitaxially deposited. The buffers were necessary to prevent nickel from diffusing into the iridium and for maintaining the epitaxy of the iridium and diamond. (2) Iridium was successfully deposited epitaxially on the $\mathrm{YSZ} / \mathrm{Y}_{2} \mathrm{O}_{3} / \mathrm{Ni}-\mathrm{W}$ substrate by electron beam evaporation. (3) A diamond nucleation process was developed for increasing the diamond nucleation density on the iridium surface. This employed a DC-biased approach in a hot filament chemical vapor deposition (CVD) system. (4) Many CVD diamond films have been deposited using both hot filament and microwave excitation of the gases. These films have been characterized via scanning electron 
and transmission electron microscopy, Raman spectroscopy, X-ray diffraction, and other techniques. (5) The DC-biased enhanced nucleation process was performed in the microwave reactor as well as in the hot filament reactor producing extremely high nucleation densities.(6) Diamond films were nucleated and grown in the microwave chamber that displayed high levels of epitaxy with the underlying iridium coatings. (7) Collaboration with Prof. Brage Golding at Michigan State University was also initiated on the growth of high quality epitaxial diamond films on the biaxially textured $\mathrm{Ir} / \mathrm{YSZ} / \mathrm{Y}_{2} \mathrm{O}_{3} / \mathrm{Ni}-\mathrm{W}$ substrates. Golding's group is a world leader in deposition of epitaxial diamond films. A few diamond films deposited by them on $\mathrm{Ir} / \mathrm{YSZ} / \mathrm{Y}_{2} \mathrm{O}_{3} / \mathrm{Ni}-\mathrm{W}$ substrates made at ORNL show almost perfect epitaxy of the diamond film with the iridium under layers, as revealed by cross section TEM and electron backscatter Kikuchi diffraction.

The results obtained to date demonstrate that the stated goals of the proposal have been achieved on a few samples. It has been discovered that the growth conditions must be very precisely controlled in order to obtain the desired results. Since the stated goals of the project have been obtained, more effort needs to be spent on determining what growth parameters are critical to control in order to obtain consistently good results.

\section{9: Scalable Surface-Enhanced Raman Spectroscopy (SSERS) for Single-Molecule Detection and Characterization}

Zhenyu Zhang, Gyula Eres, Baohua Gu, Robert N. Compton, Efthimios Kaxiras, and Wei Wang

\section{Project Description}

In this initiative, we propose an integrated theoretical and experimental effort aimed to establish the validity of one central idea: development of SCALABLE surface-enhanced Raman spectroscopy (SSERS) as a predictive analytical technique with single-molecule-detection sensitivity. The scalability is to be achieved by spatially aligning two arrays of metal nanoparticles or nanoshells self-assembled or fabricated on lithographically patterned substrates. A nanoneck distance separates each nanoparticle pair within the nanoparticle arrays, defining a local "hot spot" with respect to SERS. Collectively, the hot spots merge to define a "hot zone," rendering the device ultrahigh sensitivity for single-molecule detection. The predictive power is to be achieved via the state-of-the-art multiscale modeling, hybridizing first-principles approaches and classical electromagnetic descriptions within the generalized Mie theory. We will primarily explore the existence of collective phenomena in SERS caused by the multinanoparticle nature of the aligned geometry, and the feasibility of extracting angular information from the SERS signals. Together, the proposed research is to lay the foundation for making scalable SERS a powerful technique with ultrahigh sensitivity in single-molecule detection and characterization of chemical and biological agents, with immense application potentials in a broad range of fields from chemical, biological, and biomedical research and trace-element detection to homeland security applications. 


\section{Mission Relevance}

This work is directly relevant to DOE's research portfolio in basic science. It is particularly relevant to DOE initiatives in materials science, nano-science, and single-molecule imaging. At ORNL this work is particularly relevant to some of the research directions within the Center for Nanophase Materials Sciences (CNMS).

\section{Results and Accomplishments}

The results can be summarized as follows. (a) We showed within the framework of the generalized Mie theory that large local field electromagnetic enhancement suitable for single molecule SERS may occur due to collective phenomena. In particular, we studied onedimensional nanoshell dimer arrays. Under optimal conditions, the local electromagnetic enhancement at the nanoneck region of a nanoshell dimer in the array can be 10 times higher than that due to an isolated nanoshell dimer. (b) We proposed a new chemical contribution to SERS, caused by the dynamic polarizability of the metal substrate as it is modulated by the diffusive scattering of the electrons within the substrate by the adsorbed molecules. The modulated polarization of the substrate coupled with the incident light will contribute to the Raman scattering enhancement. (c) We studied SERS using gold nanoparticle aggregates and ordered gold nanopillar arrays. We synthesized gold colloidal nanoparticles with controlled size in a range from 5 to $100 \mathrm{~nm}$ and studied the relationship between colloidal aggregation and SERS. With controlled aggregation of gold colloidal particles, we observed a large enhancement of Raman signal. Furthermore, we developed a method to fabricate highly ordered gold nanopillar arrays using anodized aluminum oxide as a template. The ordered nanopillar arrays give reproducible SERS signals with thionine as a test molecule.

\section{Publications}

Persson, B., B., K. Zhao and Z. Zhang. 2006. Phys. Rev. Lett. 96, 207401.

Persson, B., K. Zhao and Z. Zhang. 2006. Phys. Rev. Lett. 97, 199702

Zhao, K., H. Xu, B. Gu, and Z. Zhang. 2006. J. Chem. Phys. 125, 081102.

$\mathrm{Gu}, \mathrm{B}$. and C. Ruan. 2007. "Determination of Technetium and its Speciation by SurfaceEnhanced Raman Spectroscopy.” Anal. Chem. 79, 2341-2345.

Ruan, C., et al. 2007. "Controlled Fabrication of Nanopillar Arrays as Active Substrates for Surface-Enhanced Raman Spectroscopy.” Langmuir. 23, 5757-5760.

Ruan, C., W. Wang, and B. Gu. 2007. "Single-Molecule Detection of Thionine on Aggregated Gold Nanoparticles by Surface-Enhanced Raman Scattering." J. Raman Spectrosc. 38, 568-573. 


\title{
00405: Quasielectrostatic Carbon Orientation (QCO) Processing for Lithium Ion Battery Anodes and Other Applications
}

\author{
Jane Howe, Andrew Kercher, Mina Yoon, Cliff McCold, Nancy Dudney
}

\section{Project Description}

Quasielectrostatic Carbon Orientation (QCO) processing was conceived to produce advantageously aligned carbon structures for applications, such as lithium ion batteries (LIB) and low-cost carbon fibers. The proposed QCO technique employed electric fields during key stages of thermal processing in order to produce aligned carbon materials. This technique was applied to key soft and hard carbon precursors. During thermal treatment, a strong electric field applies a torque to growing planar aromatic structures that would promote their alignment along the electric field direction. If successful, the technique would produce monolithic carbon plates and ribbons (or fibers) with graphene sheets aligned relative to the planar surface (or for fibers, relative to the fiber axis). Computer modeling was developed in parallel to provide fundamental insights into the QCO technique. If successful, carbon materials produced in this project would be tested in a LIB ion battery test cell. In LIB applications, this graphene sheet alignment would permit optimal lithium diffusion through the carbon structure, resulting in carbon anodes with higher charge rates and higher maximum current flow. Aligned hard carbon anodes should have an intermediary carbon structure between traditional hard carbons and graphite that may provide remarkably higher power density than graphite and lower irreversible capacity than traditional hard carbons. QCO processing also would have the potential to produce synthetic graphite at dramatically lower temperature $\left(\sim 1000^{\circ} \mathrm{C}\right.$ vs. $2500-3200^{\circ} \mathrm{C}$ for typical graphite), significantly lowering the manufacturing cost.

\section{Mission Relevance}

The QCO process, if proven, would benefit a number of programs and agencies. The DOE Office of Energy Efficiency and Renewable Energy would be interested in QCO for developing lightweight automotive materials and graphite electrodes for metals processing. The Department of Defense would be interested in applying the technology to lithium ion batteries for portable power applications and to low-cost, high-performance carbon fibers and heat spreaders. However, the effort of pursuing follow-on funding has yet to be successful.

\section{Results and Accomplishments}

For performing QCO processing, a parallel plate capacitor rig was constructed to apply strong AC electric fields to carbon precursors inside a tube furnace. The QCO system allowed field frequencies from $20 \mathrm{~Hz}$ to $\sim 800 / \mathrm{kHz}$ and field strengths up to approximately $6 \mathrm{kV} / \mathrm{cm}$. QCO processing has been applied to phenolic resin (hard carbon precursor) and AR mesophase pitch (soft carbon precursor) at key processing temperatures. Phenolic samples had an electric field applied up to a carbonization temperature of $650^{\circ} \mathrm{C}$. Vacuum heat-treatment was used to extend the processing temperature range of $\mathrm{AR}$ mesophase pitch up to $305^{\circ} \mathrm{C}$ without foaming.

Characterization of the phenolic resin and the AR mesophase pitch samples demonstrated no significant alignment of the microstructure for any QCO processing conditions attempted. 
Impedance spectroscopy was ineffective for evaluating microstructural alignment, because sample porosity formed during thermal processing dominated the frequency-dependent behavior of the impedance. X-ray diffraction conclusively showed that no QCO samples showed significant alignment relative to samples formed under no applied field.

Preliminary mathematical modeling indicated that electric fields below $100 \mathrm{~Hz}$ could apply torques to polarizable molecules in the samples heated under $400^{\circ} \mathrm{C}$. Computer simulations utilizing a molecular dynamics model were used to understand the field induced structural transitions which could occur during the QCO process. Two key timescales were identified: microseconds to connect individual molecules and form a knot and milliseconds to untangle and relax the knot. At low field strengths, these chain structures were tangled. Above a critical field strength, these tangled chain structures rearranged to produce straight and highly aligned chain structures.

\section{6: In Situ Monitoring of Realistic Catalyst Systems with High- Speed Electron Microscopy Using a LAB 6 Nanowire-Based Electron Source}

Niels de Jonge and Gabriel M. Veith

\section{Project Description}

We are developing a micro-reaction chamber inside a flow cell for high-resolution imaging in an electron microscope. With this system, catalytic reactions can be investigated under realistic conditions with a sub-nanometer spatial resolution and a subsecond temporal resolution. The system is constructed with an enclosed heater to observe catalytic reactions at their operating temperatures and pressures. In this seed money project, we have designed an in situ electron microscopy system that can be used for various electron microscopes; we have constructed prototype elements of the system; and we have performed the first experiments. The original project plan was changed because we were not able to conduct the experiments on electron sources.

\section{Mission Relevance}

We aim to set up a new program focusing on catalytic studies. This area had the largest proposed increases in DOE's FY 2007 budget ( \$10M for Catalysis and Chemical Transformations and $\sim 5 \mathrm{M}$ in Chemical Imaging in the Office of Basic Energy Sciences budget). There are also Energy Efficiency and Renewable Energy funding opportunities for applications such as fuel cells and automotive catalysts. In addition, this work could lead to a new "baseline" program on in situ electron microscopy from DOE.

Chemistry based on catalytic reactions forms a major part of the U.S. economy and emission controls. One example is the reduction of emissions from combustion engines using platinum catalysts. However, many catalytic reactions are not yet fully understood at a molecular level. 
Catalytic materials can be investigated before and after a reaction or during exposure at low pressures and temperatures. In operandi characterization requires a high-resolution imaging technique with true in situ capabilities. Currently, no existing systems provide imaging at molecular resolution under atmospheric pressure and high-temperature conditions.

\section{Results and Accomplishments}

During FY 2007, we designed and successfully tested a dedicated specimen holder for in situ electron microscopy. It uses a side-entry holder for a Hitachi microscope and is suitable for a transmission electron microscope (TEM) as well as a scanning TEM (STEM). The system is based on a microreaction chamber with electron-transparent windows. Gas at atmospheric pressure flows in the space between the windows, while the vacuum of the electron microscope is not compromised. Optionally, a heater can be manufactured for use on the top window using a micro-fabrication process. High-resolution images are obtained using the electron beam that is transmitted through the flow-cell/reaction-chamber. The most critical aspect of the design is the connection from the macroscale (tubing) to the microscale (in situ cell comprised of silicon chips with silicon nitride windows). Custom designed silicon chips with electron-transparent silicon nitride windows were purchased from Protochips Inc. (NC). The system was tested using a $200 \mathrm{kV}$ STEM on a sample consisting of $2 \mathrm{~nm}$ gold nanoparticles supported on a titanium dioxide layer deposited onto a silicon nitride window. Imaging was accomplished in air at 1 atmosphere pressure as well as under carbon monoxide oxidation conditions. The material was tested ex situ prior to in situ studies. The spatial resolution of the image, which was recorded over $10 \mathrm{~s}$, was approximately $1 \mathrm{~nm}$. We have also recorded sequential images at a speed of $0.2 \mathrm{~s}$ to create a movie, which involved imaging with a pixel dwell time of less than $1 \mu$ s (i.e., highspeed imaging). In this case, there were no obvious changes in the catalyst particles during these experiments due to the stability of the catalyst as a function of time under the test conditions used. Our next experiments will involve in situ imaging of catalytic reactions under representative catalyst exposure conditions.

This project plan changed because the post-doc from the University of North-Carolina at Chapel Hill decided to leave after having received 3 months of training. Filling the vacated position and training a new post-doc would have led to too long a delay to be able to obtain results in FY 2007. Therefore, the project plan was modified to obtain results on in situ imaging.

\section{7: Microstructure and Defects in Energetic Materials and Radioactive Alloys}

Gene E. Ice, Eliot D. Specht, Frederick J. Walker, John D. Budai, Bennett C. Larson, and Roger E. Stoller

\section{Project Description}

Improvement of materials depends on tailoring their defects. In particular, structural materials for high-radiation environments must retain defects such as precipitates, which provide strength while avoiding formation of defects such as dislocations that would make them brittle. 
Unintended detonation of energetic materials is thought to be initiated at lattice defects, but it is still not understood which types of defects are to be avoided. Diffuse X-ray scattering provides a sensitive measure of defect type and distribution in many materials, but safety concerns have limited its use for radioactive and energetic samples. In addition, conventional diffuse scattering measurements have required single-crystal samples, which cannot be readily obtained for many materials of interest. Using recently developed X-ray microbeams and efficient X-ray area detectors, the amount of material required for diffuse scattering measurements can be reduced from milligrams to nanograms. Irradiated samples with these volumes have much lower activity, and energetic samples fall below the critical size for detonation, so both can be handled more safely. Additionally, the X-ray beam can be focused onto a single grain in a polycrystalline sample, treating it as a small, single crystal. This project investigated whether diffuse scattering using X-ray microbeams can characterize the defects in these classes of materials.

\section{Mission Relevance}

Work on irradiated materials will support materials research in the development of nextgeneration nuclear reactors and in the ITER project, both of which are part of the American Competitiveness Initiative. Characterization of energetic materials is needed as critical input to numerically model the initiation of detonation, which will ultimately result in improved safety assessments of the handling and assembly of weapon systems, benefiting several programs funded by the National Nuclear Security Administration and the Office of Naval Research. The techniques also have wide applications to combinatorial studies of materials to understand processing and/or compositional impacts on defects.

\section{Results and Accomplishments}

In FY 2007, the project's second year, we demonstrated that diffuse scattering of X-ray microbeams can be used to characterize the defects induced by irradiation of structural materials. A polycrystalline Fe foil irradiated with protons at the University of Michigan Ion Beam Laboratory was compared with an unirradiated control. Intense Bragg scattering was found to interfere with the measurement of weaker diffuse scattering; it was found that a beamstop could be used to mitigate this problem. Diffuse scattering indicates formation of $4 \mathrm{~nm}$ clusters of interstitial atoms in the irradiated sample, while the control sample shows no evidence of defects. We found that energetic materials are not as amenable to analysis using X-ray microbeams. These materials are known to be susceptible to radiation damage and when subjected to intense $\mathrm{X}$-ray microbeams, defects induced by the X-ray probe quickly dominated the intrinsic defects of interest. Schemes for minimizing this radiation damage were evaluated. The sample was held under vacuum during analysis to eliminate reaction with air. The sample was cooled to $77 \mathrm{~K}$ to minimize the role of heat in inducing defects. The sample was analyzed using lower-intensity monochromatic X-rays rather than higher-intensity polychromatic X-rays. Despite these measures, beam-induced damage occurs before adequate scattering measurements can be made. Follow-on work will be focused on radiation effects in structural materials. 


\title{
00421: Novel, Low-Cost, High-Mn-Containing Austenitic Stainless Steels and Alloys for High-Temperature Structural Applications
}

\author{
Philip J. Maziasz, Yukinori Yamamoto, Michael L. Santella, and Michael P. Brady
}

\section{Project Description}

The purpose of this project is to design and evaluate new alloy compositions of high-Mn and Almodified austenitic stainless steels, which have both good creep resistance and good oxidation/corrosion resistance at high temperatures. High temperature strength and creep resistance is based on the unique design of a stable Fe-Cr-Mn-Ni matrix, using state-of-the-art computational thermodynamics tools to determine phase stability over a range of complex alloy compositions. Alloy design results were validated by casting trial heats of the chosen alloy compositions. The next group of alloys was made by adding high-temperature strengtheners to stable parent-phase alloys that exhibit good oxidation resistance. Due to the constraints of maintaining stable austenite parent phase, $\mathrm{Nb}$-rich intermetallics (ie., $\mathrm{Fe}_{2} \mathrm{Nb}$ or $\mathrm{Mn}_{2} \mathrm{Nb}$ ) and stable dispersions of nano-NbC carbides were used to produce high-temperature strength. Creep testing was used to screen these alloys for high-temperature strength and for oxidation resistance. High-temperature oxidation and corrosion (water vapor, sulfidation, carburization) resistance is based on additions of enough aluminum to form adherent, protective and stable $\mathrm{Al}_{2} \mathrm{O}_{3}$ surface oxide scales. This project successfully developed stable, fully austenitic stainless steel alloys that both form stable nanoprecipitate dispersions and protective alumina scales, and which still have the superior formability/manufacturability and weldability of commercial Fe-Cr-Ni austenitic stainless steels.

\section{Mission Relevance}

The success metrics and goals of this seed money project are directly related to fossil energy, and the other energy production programs, which are all trying to achieve the difficult goals of higher efficiency and cleaner energy production. This project provided unique, high-performance, lowcost Fe-Cr-Mn-Al-Ni austenitic stainless steel that can easily be substituted for conventional Fe$\mathrm{Cr}-\mathrm{Ni}$ austenitic stainless steels, which either have lower performance, or are more costly (increased Ni prices). Good high-temperature creep strength and oxidation resistance based on alumina-scales may also replace some Ni-based superalloys, which perform well, but cost far too much for many applications. These new stainless steels may also be directly applicable to efforts to extract oil from oil shale and tar sands, as well as chemical and petrochemical processing, which all also requires the most cost-effective high-performance heat-resistant and corrosionresistant stainless steel. These new stainless steels are directly or indirectly relevant to all other DOE projects requiring high-temperature alloys (e.g., Gen IV Nuclear, Transportation), and should be attractive for follow-on studies by Basic Energy Sciences because they demonstrate new, practical nanotechnology with good commercialization potential. These new low-cost, high-performance high-temperature austenitic stainless steels should also be applicable to defense programs (DoD, Defense Advanced Research Projects Agency), distributed energy for military applications (microturbines, fuel cells), and to national security or transportation applications that require more heat-resistant and failure-resistance container alloys for transporting hazardous or dangerous materials (chemicals, nuclear waste, liquid natural gas, etc). 


\section{Results and Accomplishments}

Effort began on designing these new stainless steel alloys during the last few months of FY 2006, with efforts using computational thermodynamic calculations to identify the regions of the alloy phase diagram that were $100 \%$ austenite (desired parent phase with face-center-cubic [fcc] crystal structure), with no deltaferrite or other undesirable phases. A wide area of $\mathrm{Fe}-\mathrm{Cr}-\mathrm{Ni}-\mathrm{Mn}$ Al compositions was identified, and then several alloy compositions were selected that varied only $\mathrm{Cr}, \mathrm{Mn}$ and Ni. Small heats were cast and processed into wrought plate. These new alloys were tested for microstructure, hardness, and oxidation behavior after an aging/screening test of $168 \mathrm{~h}$ at $800^{\circ} \mathrm{C}$. Most of the alloys were $100 \%$ austenite parent phase, with no ferromagnetic behavior because they contained no deltaferrite before aging, but only two of those showed good oxidation resistance, and only one showed the desired protective alumina scale formation for oxidation resistance

These base alloys were then used to define the next set of more complex Fe-Cr-Ni-Mn-Al-Cu$\mathrm{Nb}-\mathrm{C}$ alloys in FY 2007. This new group of alloys was screened by creep-rupture testing in air at $750^{\circ} \mathrm{C}$ and $100 \mathrm{MPa}$, and by oxidation testing in air at $800^{\circ} \mathrm{C}$ for $1,000 \mathrm{~h}$. Experimental alloys were made for a systematic set of base alloys, varying $\mathrm{Cr}$ from 10 to $14 \mathrm{wt} \%$, Mn from 5 to $15 \%$, and $\mathrm{Ni}$ from 4 to $12 \%$. All the alloys were made with $3 \% \mathrm{Cu}, 2.5-3.0 \% \mathrm{Al}, 0.38-0.6 \% \mathrm{Nb}$ and $0.05-0.15 \% \mathrm{C}$. The best combination of creep-rupture strength and oxidation resistance was found in a Fe-14Cr-2.5Al-12Ni-5Mn austenitic stainless steel alloy, with additions of $\mathrm{Cu}, \mathrm{Nb}, \mathrm{C}$ and $\mathrm{B}$. This alloy had creep resistance comparable to the good creep resistance found in commercial alloys like HR120, and good alumina scale formation at $700-800^{\circ} \mathrm{C}$, but with cost comparable to type $347 \mathrm{H}$ stainless steel (HR120 is about three times the cost of $347 \mathrm{H}$ steel). Another new alloy with Fe-14Cr-2.5Al-8Ni-15Mn composition was also identified, which has lower cost than $347 \mathrm{H}$ steel and still has considerable better creep resistance and oxidation resistance. The initial testing also showed good weldability, so both of these alloys are candidates for further investigations on appropriate applied DOE programs, and for commercial scale-up consideration.

The good results on these new high-Mn, aluminum-modified austenitic stainless steels were neither published nor presented this year so that the new intellectual property could be captured in an invention disclosure and patent application. ORNL ID 1976 for "High-Manganese Austenitic Stainless Steels," by M.L. Santella, Y. Yamamoto, P.J. Maziasz, and M.P. Brady was filed with ORNL and UT-Battelle on August 30, 2007. 


\title{
00422: Laser-Interference Direct Structuring of Zirconia for Dental Materials
}

\author{
Claus Daniel, Narendra B. Dahotre, and Beth L. Armstrong
}

\section{Project Description}

Adhesion of dental composite materials to the tooth surface (i.e., dentin and enamel) is a major concern for dental component lifetime. Traditional fillings fail after 6-10 years because of a gradual degradation in surface adhesion and crack initiation at the occlusal surface. To improve the lifetime of dental components, we envision a laser treatment that uses interferometry to produce periodic, microscopic surface structures that could be applied to either the tooth surface or the dental composite or both, depending on the type of filling. The novelty of using a laser to treat teeth offers the possibility of both chemically and physically nanostructuring these surfaces. Our expectation is that nanostructuring can lead to improvement in dental composites and increase the materials strength.

The specific aim of this project is to demonstrate our laser interference technique by engineering and restructuring zirconia surfaces on the micro- to nanoscale as an example of techniques that could be used for dental and other implant materials. In our technique, a primary laser beam is divided into two or more beams that are then guided by an optical system to interfere with each other at the sample surface. The standing optical wave describes a periodic intensity pattern. If our hypothesis is correct, the patterned microstructures should show improved mechanical, tribological, and biointerface properties.

\section{Mission Relevance}

The U.S. population needs implant materials with longer lifetimes than materials in current use (6-10 years for dental fillings and about 15 years for orthodontic implants). Optimizing the biointerface represents the most important factor to achieving this goal. As such, this project will benefit the National Institute of Dental and Craniofacial Research within the National Institutes of Health (NIH). Furthermore, the understanding of the phase changes, mechanical changes, and heat transportation will benefit the DOE Office of Science, Basic Energy Sciences program. Once the possibilities and limitations are understood, this technique can provide unique periodic microstructures and diffusion profiles that are not achievable using other techniques.

Conceivably, the microstructured surfaces could also show improved tribological properties for automotive, space, and industrial applications. Therefore, this project may also benefit the DOE Office of Energy Efficiency and Renewable Energy.

\section{Results and Accomplishments}

\section{FY 2006 Accomplishments:}

Yttria-stabilized zirconia pellets were prepared using both tape casting/lamination and pressing techniques. Due to the laser treatment, the near-surface microstructure could be significantly changed without any measurable ablation of the material. The initial grain size of about 1 to $2 \mu \mathrm{m}$ could be scaled down to 10 to $20 \mathrm{~nm}$ within a surface layer of about $0.5 \mu \mathrm{m}$.

Crystallographic texture and phases are changed and will be further analyzed. The interference 
setup could be successfully installed and periodic micro-structural changes with micrometer spacing could be achieved on spot sizes with a diameter of $5.9 \mathrm{~mm}$.

\section{FY 2007 Accomplishments:}

Review comments on a National Institutes of Health proposal criticized the lack of dental expertise in our research team. In response to those, we established an informal nonfunded collaboration with James Drummond, Department Restorative Dentistry, University of Illinois, Chicago, and Russell Giordano, Goldman School of Dentistry, Boston University.

We changed our material from in-house fabricated nondental-grade zirconia to dental-grade zirconia, alumina, and feldspathic porcelain produced at Boston University. After our laser treatment, specimens were tested according to dental procedures at University of Illinois. Upon correct laser parameters, the three-point bending flexure strength could be increased by up to $50 \%$ from $833 \mathrm{MPa}$ to $1,250 \mathrm{MPa}$ in the case of zirconia and up to $40 \%$ from $422 \mathrm{MPa}$ to $630 \mathrm{MPa}$ in the case of alumina. This shows a lower crack initiation and indicates a strengthening of the occlusal surface potentially resulting in a lifetime improvement of these dental restorative materials. A patent was filed by UT-Battelle, and NIH joint proposals are under preparation.

\section{Publications}

Harimkar, S. P., et al. 2007. "Validation of Crystallographic Correlation for Faceted Morphology in Laser Surface Engineered Alumina Ceramic.”Scr. Materialia. 57, 401-404.

\section{3: Organic Magnets: Phenomenological and First-Principles Approaches to Layered Bimetallic Oxalates}

R. S. Fishman and F. A. Reboredo

\section{Project Description}

Organic magnets (also called molecule-based magnets) are a broad class of materials that have come under increasing scrutiny during the past 15 years. While organic magnets exhibit some of the same variety of behavior found in more conventional magnets, they also pose unique challenges and opportunities. Although experimental work on organic magnets has been quite active, fundamental understanding of these materials has lagged far behind. We have applied modern phenomenological and first-principles approaches to one particularly intriguing system of organic magnets: layered bimetallic oxalates. Completion of this project will lay the groundwork for a more wide-ranging project to study the general class of organic magnets.

\section{Mission Relevance}

The advanced state of organic synthesis and the wide range of potential applications make organic magnets quite attractive to both fundamental and applied scientists. Many organic magnets are functional materials that display a remarkable interplay between magnetic, electrical, and optical properties. As a result, they can play important roles in many areas of 
interest to DOE. Organic magnets like the bimetallic oxalates have potential applications as sensors and coatings.

\section{Results and Accomplishments}

During the past 6 months, we have successfully developed a phenomenological theory of the $\mathrm{Fe}(\mathrm{II}) \mathrm{Fe}(\mathrm{III})$ bimetallic oxalates. By studying the behavior of the orbital-doublet ground state produced by a $\mathrm{C}_{3}$-symmetric crystal field, we have constructed a reduced Hamiltonian that contains both exchange and spin-orbit interactions. This Hamiltonian is used to explain all of the important behavior of this class of materials, including the stability of magnetic order in wellseparated layers and the magnetic compensation (the cancellation of the magnetization on the two sublattices) in compounds with high transition temperatures. We have predicted that the negative magnetization can be optically flipped by near-infrared light. Breaking the $\mathrm{C}_{3}$ symmetry about each of the $\mathrm{Fe}$ (II) ions either through a cation-induced distortion or uniaxial strain in the plane of the bimetallic layer is predicted to increase the magnetic compensation temperature $\mathrm{T}_{\text {comp. }}$ This work is scheduled to appear in Physical Review Letters.

We have also theoretically explained the unique minimum in the coercive field in a polycrystalline sample of $\mathrm{Fe}$ (II)Fe(III) bimetallic oxalates at $\mathrm{T}_{\text {comp. }}$. All other known bulk ferrimagnets exhibit a maximum coercivity at $\mathrm{T}_{\text {comp. }}$. But due to the magnetization perpendicular to the anisotropy axis, a polycrystalline system with uniaxial anisotropy (like the bimetallic oxalates) will exhibit a minimum coercive field at $\mathrm{T}_{\text {comp }}$.

\section{8: Can the Quantum Confinement Effect Be Exploited for Spin Injection in Organic Spintronics?}

Jian Shen, Chengjun Sun, and Xiaoguang Zhang

\section{Project Description}

For spintronics applications, organic semiconductors are particularly attractive because the spin scattering rate is orders of magnitude smaller than that of ordinary inorganic semiconductors. The major technical challenge in organic spintronics devices is to achieve efficient spin injection. We seek to achieve a possible $100 \%$ spin polarization of quantum well states in magnetic nanodots to boost the efficiency of spin injection. For this proof-of-principle study, we will fabricate a prototype spin valve device that uses Co nanodots and a manganite thin film as two ferromagnetic electrodes and an organic molecule layer as the spacer layer. Scanning tunneling microscopy will be used to measure local tunneling magneto-resistance and determine spin injection efficiency as a function of the size of Co nanodots, bias voltage, and temperature. Our goal is to optimize the efficiency of spin injection and the magnitude of magneto-resistance, which can have a substantial impact on future technology. 


\title{
Mission Relevance
}

While this project focuses on providing a fundamental understanding of how to control spin injection in organic spintronics, we fully anticipate that it will grow into a much broader program with an impact on diverse technologies. The novel application of organic spintronics in electroluminescence and photovoltaics could spearhead a new direction in the effort of "Scienceto-Energy" using organic spintronics devices, which is high in the agenda of both DOE and ORNL. Hence, this work would also provide a basis for use-inspired basic materials research related to energy (such as the areas of photovoltaics and solid-state lighting). Much of the spintronics research in the United States is presently funded through Defense Advanced Research Projects Agency, National Aeronautics and Space Administration, Air Force, and Army. It is likely that this work will further advance the realization of flexible spintronics and flexible electronics in general and lead to future programs that will comprehensively investigate the effects of spin injection and transport on (1) spin-polarized excitons dissociation (photovoltaics), (2) spin-polarized charge trapping and releasing (nonvolatile memory), (3) spin polarized electron-hole recombination (EL), (4) new materials as electrodes for efficient spin injection and transport, and (5) correlation of spin transfer with interfacial contact.

\section{Results and Accomplishments}

In FY 2007, we have fabricated organic spin valves in the architecture of $\mathrm{LaSrMnO}_{3} / \mathrm{MEHPPV} / \mathrm{Co}$ nanodot with $\mathrm{Al}$ as cover layer. The tunneling magnetoresistance measurements indicated that the spin injection from Co nanodots to organic semiconductor MEHPPV has been achieved, reflecting that the fabricated devices are suitable for studying the spin injection from a single Co nanodot using scanning tunneling microscope. On the theory side, we have shown that when quantum well $(\mathrm{QW})$ resonances are important, they have an opposite effect on spin-dependent tunneling for a magnetic QW state than on a nonmagnetic QW state. We demonstrated that in the case of a statistical distribution of nanodots, it is more appropriate to apply the sequential tunneling model based on a continuous density of states, than the resonant tunneling model with a set of discrete energy levels.

\section{9: In Situ Nanopatterning of Single-Crystal Multiferroics by Strain for Terabit-Scale Data Storage}

\author{
Ho Nyung Lee and Matthew F. Chisholm
}

\section{Project Description}

During the past 50 years, a tremendous, steady progress in hard disk technologies has taken place. However, the current technique for increasing the storage density over the terabit scale will meet a limitation within a few years, due to the materials' intrinsic behaviors, including the thick magnetic domains. Although continued improvement in conventional semiconductor designs to decrease the length scales has to some extent addressed these issues, increasing motivation exists to consider alternatives to patterning materials in nanoscale. In this project, we propose an innovative approach to pattern nanostructures by using so-called vacuum etching. We 
will focus mainly on the investigation of the feasibility and underlying mechanism of vacuum etching. The outcome of this work is expected to be especially useful for patterning singledomain nanomaterials and thus for applying such nanomultiferroics to ultrahigh-density information storage and nonvolatile memory.

\section{Mission Relevance}

Single-crystal nanostructures can offer rich scientific opportunities. For instance, our singlecrystal nanomultiferroics proposed here are of great interest for realizing high electromagnetic coupling by epitaxial strain and nanostructuring. Moreover, the materials to be investigated are highly relevant to DOE missions because of their potential usefulness for materials in various applications, including information storage, sensors, and actuators. Therefore, the work proposed here is an ORNL opportunity for world leadership in understanding yet unveiled physical properties of single-crystal nanoferroics. The quest for multifunctional multiferroics will contribute to a broad range of basic research programs. Our innovative approach to patterning nanocrystals will potentially benefit industry and federal agencies such as DoD's Defense Advanced Research Projects Agency, since the multiferroics are very promising for many technical applications, such as sensors, energy and information storage, and piezoelectric devices.

\section{Results and Accomplishments}

The following tasks (completed during FY 2007) provide the experimental foundation for the ambitious goals of this program: (1) the preparation of atomically flat, single-stepped crystalline films on strontium titanate $\left(\mathrm{SrTiO}_{3}\right)$ substrates as required for patterning; (2) the growth of high quality, highly polar lead zirconate titanate $(\mathrm{PZT})$ and bismuth ferrite $\left(\mathrm{BiFeO}_{3}\right)$ films that will form an integral part of functional heterostructures; (3) the production of irregular shaped, nanometer-sized islands by vacuum etching, confirming that the concept of vacuum etching works.

\section{4: Photon-Assisted Thermoelectric Devices}

Hsin Wang, David Mandrus, and David Singh

\section{Project Description}

The objective of this study is to experimentally determine the effect of photo current on thermopower and thermoelectric performance for two wide-band gap semiconductors and selected thermoelectric materials. Theoretical study will also be conducted to understand the fundamental interaction of photons and electrons in these thermoelectric materials. It will predict and specify the class of materials where photons will have the greatest enhancements of thermoelectric properties. The main purpose is to develop a good understanding of the photon effect in thermoelectric and use the knowledge to develop new thermoelectric research programs funded by DOE and other agencies. 


\section{Mission Relevance}

The recent DOE Office of Basic Energy Sciences solar energy initiative recognized the potential benefit from a thermoelectric generator. A specific solar thermal energy conversion area was identified in the initiative. If the combined thermoelectric and solar cell can be utilized, the two important functions will benefit each other. On one hand, thermoelectric devices can convert unused heat into power. On the other hand, extra light can enhance the performance of the thermoelectric devices. The effort of this project will help to improve energy conversion efficiency. In the DOE Energy Efficiency and Renewable Energy programs, alternative energy sources are the main focus. Thermoelectric materials are of particular interests because they can convert heat directly into electricity with no moving parts. Recent EERE programs include waste heat recovery from automobiles and waste heat power generation from energy-intensive industries, such as glass plants.

\section{Results and Accomplishments}

Theory: Transport calculations as a function of temperature and doping were performed for $\mathrm{ZnO}$. These were based on first principles, density functional calculations of the electronic structure using a very fine Brillouin zone sampling, as is needed for transport. We find a strong asymmetry between electron and hole transport with a nearly order of magnitude difference in the high temperature Seebeck coefficients for electrons and holes at carrier concentrations in the 0.01 to 0.05 / f.u. range. In addition, we find a small anisotropy with slightly higher thermopower along the hexagonal axis relative to the basal planes for p-type carriers. This does not yet identify $\mathrm{ZnO}$ as promising for a photo-thermoelectric energy converter, but it does suggest a new spectroscopy that may be important. In particular, measurement of the charge collection in a thermoelectric device as a function of photon energy may yield detailed information about carrier traps in p-type $\mathrm{ZnO}$. This may be helpful in understanding the difficulties in obtaining high-mobility p-type $\mathrm{ZnO}$.

Experiments: $\mathrm{ZnO}$ single crystals in needle shape were obtained. Polycrystalline disks were obtained from $\mathrm{ABB}$. The samples we obtained were pure $\mathrm{ZnO}$. The samples were machined into prismatic bars and disks. The disks were exposed under UV light during electrical resistivity measurements using an in-line probe station. We found the resistivity changed from mega-ohms to several tens kilo-ohms. However, we also discovered the pressure contact electrodes were not making ohmic contact with $\mathrm{ZnO}$. A Schottky barrier formed between the electrode and $\mathrm{ZnO}$ and prevented further measurements. In order to prevent this, Au-Pd films were deposited at the contact area.

Electrical resistivity was measured on the ZEM-2 system. We found linear I-V relationship in the light OFF condition. When the UV lamp was turned on, resistance was measured every 10 seconds. The resistance of the specimen decreased exponentially, indicating the electrons in the traps were excited to the conduction band. We found there is a time constant in the conductivity increase under the light $\mathrm{ON}$ condition. As soon as we turn off the light, the resistance of the specimen went back up. We also measured the Seebeck coefficient of the material under the light OFF condition. The measurements are consistent with expected values for $\mathrm{ZnO}$. Under the light ON conditions, the initial results indicated a very small change in electrical conductivity. The increase in electrical conductivity could enhance thermoelectric performance and result in a 
higher figure of merit ZT. More detailed Seebeck measurements will be completed in the early part of FY 2008.

\title{
00445: Photocatalytic Conversion of $\mathrm{CO}_{2}$ : An Alternative to Storage- Based Sequestration
}

\author{
C. K. Narula, M. Moses-DeBusk, and A. C. Buchanan
}

\section{Project Description}

The state-of-the-art technology for $\mathrm{CO}_{2}$ emissions remediation involves chemical capturing techniques using aqueous amines. However, this approach is capital intensive because it requires collection of $\mathrm{CO}_{2}$ emissions at the source, transportation, and delivery to geological formations. An alternative to $\mathrm{CO}_{2}$ storage is to convert $\mathrm{CO}_{2}$ to hydrocarbons that can be used as fuel for transportation and as chemical building blocks instead of using oil derived precursors. The goal of this proposal is to explore new classes of photocatalysts for $\mathrm{CO}_{2}$ conversion to useful chemicals that can potentially function in visible light and exhibit high turnover frequencies. A perfect example of this approach is the natural process of plants converting $\mathrm{CO}_{2}$ and water to carbohydrates using photosynthesis (employing solar energy); plants then use carbohydrates as a source of energy (respiration) and break them down to $\mathrm{CO}_{2}$. This process is $\mathrm{CO}_{2}$ neutral. $\mathrm{A} \mathrm{CO}_{2}$ chemical cycle is highly desirable, provided the source of energy for reduction is renewable such as solar energy.

\section{Mission Relevance}

Successful results are directly relevant to DOE's Fossil Energy (FE) program for $\mathrm{CO}_{2}$ sequestration. The DOE-FE $\mathrm{CO}_{2}$ sequestration program focuses on advancing the technology of $\mathrm{CO}_{2}$ collection at the source and storage in geological formation; as a result, this approach is becoming a viable option for reducing the $\mathrm{CO}_{2}$ in the atmosphere. The goal of this proposal is to obtain preliminary results on photocatalysts that demonstrate improved efficiency for $\mathrm{CO}_{2}$ conversion. That data will form the basis for a proposal in response to an anticipated multimillion dollar call for proposals from DOE's Fossil Energy program for $\mathrm{CO}_{2}$ sequestration. The DOE-FE program considers photocatalytic reduction of $\mathrm{CO}_{2}$ as one of the methods for $\mathrm{CO}_{2}$ sequestration, even though there are currently no funded projects on this topic in the DOE-FE portfolio. It is important to note that the DOE Office of Science and Defense Advanced Research Projects Agency have issued calls on solar energy utilization, and we will explore funding possibilities from these organizations also.

\section{Results and Accomplishments}

We are on track to achieving all of the objectives of this project in FY 2008. We initiated this work by synthesizing $\mathrm{SiO}_{2}-\mathrm{TiO}_{2}$ catalysts with identical mesoporous substructures and $\mathrm{Si}$ Ti ratio but with different coordination of $\mathrm{Ti}$ (four and six). The catalyst with 4-coordinate Ti was synthesized employing a template-assisted sol-gel method. The catalyst with 6-coordinate Ti was synthesized by impregnation of titanium isopropoxide on mesoporous silica synthesized by a 
template-assisted process. The catalysts were characterized by standard techniques and evaluated for $\mathrm{CO}_{2}$ photochemical reduction employing a UV source. The analysis of the reaction mixture clearly showed the presence of methanol. The synthesis and characterization of binary catalyst systems are progressing well. A new catalyst system, $\mathrm{GaN}-\mathrm{ZnO}_{2}$, has recently been shown to photo-catalytically dissociate water to produce hydrogen. We are synthesizing this catalyst system by synthesizing zinc oxide with atomic distribution of gallium because we believe that this approach will lead to high dispersion of $\mathrm{GaN}$ in a $\mathrm{ZnO}_{2}$ matrix, thereby, creating a larger number of sites for photodissociation. We will obtain $\mathrm{CO}_{2}$ photochemical reduction data on binary catalysts during FY 2008.

\title{
00447: In Situ Studies for Ductility Improvement of Bulk Metallic Glasses
}

\author{
C. H. Hsueh, P. F. Becher, and C. T. Liu
}

\section{Project Description}

Although bulk metallic glasses (BMGs) constitute a new class of metallic materials with extremely high strength, they often deform by the formation of localized shear bands and display little plasticity before catastrophic fracture at room temperature. This inability to permanently deform limits the use of BMGs in engineering applications. To improve the ductility of BMGs, particle reinforcement has been attempted. However, both ductility enhancement and embrittlement have been reported for BMG composites subjected to compression tests. Also, it appears that reinforcing particles have little effect in improving the ductility in tension. To resolve this controversial issue, it is imperative to understand how shear bands interact with reinforcing particles. Also, shear fracture of BMGs has been found to depend not only on the shear stress but also on the stress normal to the shear plane, and the Mohr-Coulomb criterion, an empirical equation accounting for the effects of normal stresses on shear fracture, has been adopted to characterize shear fracture of BMGs. However, systematic verification of the MohrCoulomb criterion for BMGs is nonexistent because of the difficulty of controlling the normalto-shear stress ratio on the shear plane in the existing tests. Hence, we proposed to use a cleverly designed test fixture that allows one to control the location of shear fracture and the normal-toshear stress ratio on the shear plane. This would provide for in-situ observation of the interaction between shear bands and reinforcing particles during shear fracture of BMGs. It would lead to a mechanistic understanding of ductility enhancement and provide a basis for modeling/predicting particle reinforcement in BMGs. The fixture would also provide a means to verify the applicability of the Mohr-Coulomb criterion for BMGs. Furthermore, while BMGs are known to be subject to strong residual stresses because of the high cooling rate during the solidification process, studies of effects of residual stresses on mechanical properties of BMGs are lacking. Because the residual stress has a certain distribution profile in BMGs, controlling the location of shear fracture enables the systematic study of effects of residual stresses on shear fracture.

Success of this research would lead to a predictable way of improving the ductility of BMGs using particle reinforcement. A variety of uses of BMGs has been identified, including sport and 
luxury goods, and electronic, medical, and defense applications. The potential funding sources include the Office of Naval Research, the Army Research Laboratory, the Air Force, and the Defense Advanced Research Projects Agency. Also, completion of our proposed work would establish proof of principle that our test fixture has the capabilities of controlling the location of shear fracture and the normal-to-shear stress ratio on the shear plane, which cannot be achieved by existing tests performed on BMGs. These capabilities can extend our proposed work to systematically characterize how the residual stresses can be utilized to improve the ductility of BMGs and verify the applicability of the Mohr-Coulomb criterion for BMGs which, in turn, will generate funding opportunities from basic programs (e.g., Basic Energy Sciences and the National Science Foundation).

\section{Results and Accomplishments}

During FY 2007, we made significant progress in four areas. (1) We were successful in designing, fabricating, and demonstrating the test fixture using hardened $440 \mathrm{C}$ stainless steel that allowed us to achieve the extremely high applied stresses required to initiate shear bands in BMGs. More importantly, we demonstrated that we could successfully control shear band formation and shear fracture of BMGs using the fixture. (2) Our work has attracted interest from other researchers, E. P. George and H. Bei, to join our study. (3) Based on our preliminary results, we have submitted a proposal entitled "Shear Deformation and Fracture of Bulk Metallic Glasses" to the National Science Foundation (Proposal Number 0800374) requesting \$383,185 for 3 years. (4) A paper entitled "Controlled Shear Bands and Shear Fracture in Bulk Metallic Glasses" has been drafted and will be submitted for publication soon.

\section{9: Assessment of Possible Exotic Magnetic Behavior in Anti- Perovskite Nitride}

James R. Thompson, Michael P. Brady, Joachim H. Schneibel, David Singh, and E. Andrew Payzant

\section{Project Description}

Preliminary experimentation had shown unexpected magnetic behavior in a thermally nitrided $\mathrm{Cr}_{3} \mathrm{Pt}$ sample, suggesting possible exotic ferromagnetic behavior by the anti-perovskite phase $\mathrm{Cr}_{3} \mathrm{PtN}$. However, the available samples were not homogenous, precluding identification of the source of the ferromagnetic behavior. The specific objectives of this small Seed proposal were (1) to synthesize phase pure $\mathrm{Cr}_{3} \mathrm{PtN}$ and (2) to evaluate its magnetic properties. The project encompassed a structural, magnetic, and theoretical study of the material. In addition, dozens of other antiperovskite nitride phases of the type $\mathrm{A}_{3} \mathrm{BN}$ have been predicted; their potential for spintronic, superconductive, and electronic applications makes them highly interesting topics for future investigation. 


\section{Mission Relevance}

The work expands DOE's extensive efforts under the Basic Energy Sciences program devoted to understanding, synthesis and functional behaviors of materials, particularly complex oxides. There is, however, comparatively little effort devoted to complex nitrides, which comprise an emerging area of interest. Their potential for novel spintronic, superconductive, and electronic properties may lead to applications in energy utilization and efficiency.

Discovery of novel forms of magnetic materials would be beneficial to the DoD for sensors, actuators, and potentially data storage. The Office of Naval Research has a long-standing program on structure and properties of novel materials.

\section{Results and Accomplishments}

During the 4-5 month duration of this project, samples of the antiperovskite nitride compound $\mathrm{Cr}_{3} \mathrm{PtN}$ were synthesized and shown to be phase-pure, as indicated by X-ray diffractometry confirming their antiperovskite structure. Analysis of elemental content showed the material to have the ideal stoichiometry to within $1.5 \%$. Magnetic studies, using a superconducting quantum interference device (SQUID)-based magnetometer, revealed a weak ferromagnetism with a Curie temperature near $110 \mathrm{~K}$. The presence of ferromagnetism is consistent with theoretical calculations of the spin-polarized band structure; however, the experimentally observed saturation magnetization is anomalously low compared with that ordinarily expected from $110 \mathrm{~K}$ ferromagnetic transition temperature, implying a possible exotic origin. The observed magnetization is also significantly smaller than the value calculated theoretically for ideally ordered and stoichiometric $\mathrm{Cr}_{3} \mathrm{PtN}$.

We also investigated the possibility of synthesizing several chemically related antiperovskites, including a replacement of $\mathrm{Pt}$ with either $\mathrm{Pd}$ or $\mathrm{Ni}$. The compound $\mathrm{Cr}_{3} \mathrm{PdN}$ was attained, while the more dissimilar Ni-based analog did not form under the available processing conditions. Magnetic investigations of the Pd-based material showed it to be paramagnetic, thereby confirming that the observed ferromagnetism in the Pt-based anti-perovskite nitride is specific to that material.

\section{4: An Innovative Low/High-Temperature, Repetitive Pressure- Pulse Apparatus for Cavitation Damage Research}

John Wang, Narendra Dahotre, Seokho H. Kim, Peter Blau, and Steve Pawel

\section{Project Description}

Cavitation damage has manifested itself as a major obstacle in many advanced technological developments either at room or at high temperatures (e.g., in spallation sources, modern highspeed turbomachinery, rocket engines, gas and steam turbines, diesel engines for heavy vehicle propulsion, spacecraft and high-speed marine vehicles, commercial power generating systems, propellers, pumps, bearing components). We propose to demonstrate the feasibility of a novel, 
laser-assisted repetitive pressure-pulsed apparatus to generate controllable cavitation events. In support of this demonstration, we will develop models to determine the cavitation parameters, such as pressure magnitude, and estimate the cavitation damage. This undertaking will support efforts to elucidate some of the fundamental hydraulic-mechanical processes associated with cavitation and its effects on target materials. With the main focus on the controllable cavitation parameters, such as pressure magnitude and temporal characteristics, as well as on damage characterization, the research carried out will have a wide-ranging impact on several important industrial sectors and their supply chains.

\section{Mission Relevance}

The success of this project will greatly increase our understanding of the underlying mechanisms of cavitation damage and enable the development of strategies to prevent or mitigate this form of damage.

This work will impact programs within the DOE and DoD that focus on advanced engine development, advanced materials development, and their effective lifetime estimates when subjected to cavitation damage. For example, mercury target cavitation in the DOE Spallation Neutron Source (SNS), potential plasma cavitation in ITER (International Thermonuclear Experimental Reactor) fusion reactor environment, the Navy fleet and aircraft integrity surveillance, the National Aeronautics and Space Administration space propulsion program, the DoD gas turbine engine development, the DOE Nuclear Energy program on Nuclear Power Reactor System, and the DOE Heavy Vehicle Propulsion program.

\section{Results and Accomplishments}

In 2007 an experiment was conducted using aluminum foil in water to investigate the cavitation damage from laser-pulse induced pressure waves. The experimental setup consists of an openloop system design and an optical microscope with resolution of about $0.7 \mu \mathrm{m}$ to observe bubbles formation. The interferometer beam was combined with the optical microscope for surface monitoring of the acoustic (pressure) wave amplitude and waveform in the frequency bandwidth of $4.5 \mathrm{GHz}$. The tested samples were placed in a glass container filled with water. A $1,064 \mathrm{~nm}$ YAG laser beam was focused using a cylindrical lens; the optical breakdown near the focus region then forms the shock waves and bubbles. The maximum laser energy used in this experiment was $200 \mathrm{~mJ} /$ pulse, with pulse duration of 5-7 ns and repetition rates ranging between 0.5 and $20 \mathrm{~Hz}$. The pulse wave volume was about $5 \times 5 \mathrm{~mm}$ in cross-section and about $100 \mu \mathrm{m}$ in thickness. Significant bubbles were formed initially between the laser plasma and the test sample surface, and much less bubble density elsewhere. Significant cavitation damage was observed on the aluminum foil samples that were processed at a repetition rate of $20 \mathrm{~Hz}$ for 20 min. A higher-power laser will be used for the proposed research in 2008, which will be focused on designing a test protocol and to generate cavitation damage on stainless steel and aluminum alloys. 


\title{
00456: High-Strength, Ductile, Crystalline-Amorphous Multilayers
}

\author{
T.G. Nieh
}

\section{Project Description}

It has been recently shown that nanostructured $\mathrm{Cu} / \mathrm{Nb}$ multilayers, particularly those with layer thicknesses of a few nanometers $(\sim 4 \mathrm{~nm})$, have a high strength and are extremely resistant against blistering induced by He-ion irradiation. The remarkable structural stability and high strength was attributed to the presence of a high density of coherent interfaces (a hetero fcc/bcc interface). Bulk metallic glass (BMG) is also known to be very strong but extremely brittle in tension, as a result of the formation of highly localized shear bands. However, our recent results of the study of localized shear band formation in metallic glasses indicated that when the characteristic length of an amorphous phase is smaller than the size of a shear-band nucleus, shear band nucleation cannot take place and catastrophic failure will be prevented. It is, therefore, anticipated that an amorphous-crystalline multilayered structure with the amorphous layer thinner than the shear band nucleus size, which is $\sim 10 \mathrm{~nm}$, will exhibit a high strength and high tensile ductility. In this project, we propose to fabricate a $\mathrm{Cu}-\mathrm{Zr}$ nanolayer to demonstrate this conjecture.

\section{Mission Relevance}

The DOE Office of Basic Energy Sciences has long, genuine interest in the basic research for the understanding and development of high-strength, high-ductility materials. By demonstrating that crystalline-amorphous nanolayers can be fabricated with a good balance of strength and ductility, we will be well positioned to propose to BES an innovative technique to synthesize new structures in a controlled fashion that will enable fundamental research on mechanical behavior and defect mechanisms in thin film and layered structures. Eventually, because of their potential for greatly enhanced irradiation resistance, these materials may also be of interest to applied programs.

\section{Results and Accomplishments}

During the period of performance in FY 2007, we made significant progress in three areas. (1) We prepared free-standing $\mathrm{Cu}-\mathrm{Zr}$ nanolayers $(\sim 50 \mu \mathrm{m}$ in the total thickness) by radio frequency magnetron sputtering and subsequently annealed it to induce solid-state amorphization between $\mathrm{Cu}$ and $\mathrm{Zr}$. The resultant nanolayered structure consists of alternative amorphouscrystalline nanolayers, with the individual thicknesses of the crystalline $\mathrm{Cu}$ and amorphous $\mathrm{Cu}$ $\mathrm{Zr}$ layers to be about 38 and $5.5 \mathrm{~nm}$, respectively, equivalent to about $12.5 \mathrm{vol} \%$ of the amorphous phase. (2) The $\mathrm{Cu}-\mathrm{Zr}$ nanolayer shows yield strength of $420 \mathrm{MPa}$ and tensile strength of 1,120 MPa. In comparison, the reported yield and tensile strengths of a bulk nanocrystalline $\mathrm{Cu}($ nano- $\mathrm{Cu}$ ) with an average grain size of $30 \mathrm{~nm}$ are 450 and $725 \mathrm{MPa}$, respectively. The most notable feature of the nanolayer is the remarkable tensile elongation of $\sim 4 \%$, as we originally conjectured. (3) The strength of the nanolayer is much higher than that predicted by the conventional isostrain model $(1,120 \mathrm{MPa}$ vs $850 \mathrm{MPa})$, indicating there is a strong interaction between the crystalline $\mathrm{Cu}$ and amorphous $\mathrm{Cu} / \mathrm{Zr}$ layer. A study of this type of interaction will 
shed light on the dislocation-grain boundary interaction because, structure-wise, there is a great similarity between a grain boundary and an amorphous phase.

\section{8: Deterministic Growth of Oxide Nanostructures by Pulsed- Laser Deposition}

Hans M. Christen, Gyula Eres, and John E. Mathis

\section{Project Description}

Complex oxide materials provide a wealth of well-known, unique, and useful properties.

However, due to the fundamental lack of methods suitable to process these oxides into nanoscale devices, these properties have not been exploited in actual applications. An alternative is needed to construct nanoscale oxide structures from the bottom up (i.e., to apply patterning techniques to masks and catalysts and then grow the desired nanostructures at the predetermined locations), eliminating the need of postprocessing. Pulsed-laser deposition (PLD) has been highly successful in the synthesis of a broad variety of oxide materials of highest quality in thin film and superlattice forms. In this proposal we address the challenge of directly synthesizing metal-oxide nanorods and nanorod assemblies by exploiting the highly non-equilibrium processes in PLD to generate the prerequisite growth selectivity.

\section{Mission Relevance}

The work of this project relates to a number of DOE goals and missions, with energy-related applications motivating many of them. In particular, solar energy conversion (photovoltaic and solar fuel) will benefit strongly from the anticipated results. Here, using epitaxial nanorods allows otherwise mutually exclusive requirements (long photon absorption path, short electron extraction length) to be combined. Other energy-related applications, such as increased flux pinning in superconducting tapes, and their potential use as catalyst supports, are also of great relevance to Basic Energy Sciences and Energy Efficiency and Renewable Energy.

\section{Results and Accomplishments}

During the first month of the project (FY 2007), initial experiments to grow nanorods of magnesium oxide, zinc oxide, and lanthanum aluminum oxide, have been carried out, all using gold nanoparticles as nucleation sites. These structures have been analyzed by scanning electron microscopy and atomic force microscopy. The FY 2008 work will focus on the characterization of nanorod heterostructures and their analysis by transmission electron microscopy and physical properties measurements. 


\title{
00452: Fundamental Studies of $\mathrm{CO}_{2}$-Coal Interactions Using Novel Neutron-Scattering Techniques at Conditions Relevant to Subsurface Sequestration
}

\author{
Y. B. Melnichenko, G. D. Wignall, D. R. Cole, A. Radlinski, and M. Mastalerz
}

\section{Project Description}

Carbon dioxide $\left(\mathrm{CO}_{2}\right)$ is the greenhouse gas that makes the largest contribution to global warming, and roughly one-third of the United States' $\mathrm{CO}_{2}$ emissions are generated by fuelburning power plants. Capture and storage of $\mathrm{CO}_{2}$ in underground geologic structures may significantly reduce $\mathrm{CO}_{2}$ emissions to the atmosphere. Sequestration of $\mathrm{CO}_{2}$ in unmineable deep coal seams is particularly attractive as many coal-burning power plants are located near sites potentially suitable for geological storage. This research applies the small-angle neutron scattering (SANS) technique to study the $\mathrm{CO}_{2}$-coal interactions and structure modification of several reference samples of coal at temperatures and pressures similar to those found in deep coal seams, which are likely to be used for industrial-scale underground storage of $\mathrm{CO}_{2}$. ORNL has extensive experience and expertise in applying SANS to investigate the phase behavior of fluids in engineered porous materials. These capabilities will be extended to studies of confined fluids in natural porous structures and enhanced by employing the new state-of-the-art SANS instrumentation that will soon become available at the High Flux Isotope Reactor and the Spallation Neutron Source.

\section{Mission Relevance}

This research is relevant to the core research activities in geosciences research (chemical sciences, geosciences, and biosciences) and and in neutron and X-ray scattering (materials sciences and engineering) within the DOE Office of Science Basic Energy Sciences program. It supports the President's clean coal initiative "to advance technologies that can help meet the nation's growing demand for electricity while simultaneously providing a secure and low-cost energy source and protecting the environment." The practical implications of this work may also be of a great value to select projects within the Office of Fossil Energy's carbon sequestration research portfolio. This study will help to understand the reasons of variable $\mathrm{CO}_{2}$ injectivity at different storage sites observed during the field tests. The ability to predict the effectiveness of $\mathrm{CO}_{2}$ sequestration is important for economically viable sequestration practice which may contribute to the reduction of the greenhouse emissions and thus improve environmental quality in the United State and the world.

\section{Results and Accomplishments}

The project started late in FY 2007. We obtained and shaped samples of coals from the Illinois basin as well as the Bowen basin in Australia and conducted two SANS and USANS experiments in order to explore the influence of pressurized $\mathrm{CO}_{2}$ on the structure and interactions with the chosen coals. Experiments revealed several unexpected effects such as plasticization of 
the coal matrix with $\mathrm{CO}_{2}$, uneven distribution of the absorbed fluid over the coal volume, and fast kinetics of the coal saturation. The preliminary analysis of the SANS and USANS characterization of the $\mathrm{CO}_{2}$ adsorption was summarized and presented at a workshop in Brisbane, Australia. 


\title{
00412: A Novel Radio-Luminescent Glass Designed for Safe User Applications
}

\author{
C. W. Alexander, R. W. Smithwick, Linda Lewis, and L. A. Boatner
}

\section{Project Description}

The goal is to develop a new approach for producing radio-luminescent materials in which the radioactive activator is physically incorporated in the bulk of a glass phosphor. The incorporation of the activator will be achieved by in situ irradiation of ${ }^{6} \mathrm{Li}$ (lithium) to produce a ${ }^{3} \mathrm{H}$ ion (tritium) upon exposure to a high neutron flux. Incorporation of the tritium will facilitate a $360^{\circ}$ luminescence activation region within the glass phosphor. Such a configuration is expected to greatly increase the luminescent output and create a radiologically safe material that may be applied to both bulk and nano-sized materials. The final product will be (1) self powered by the incorporated tritium, (2) highly luminescent, (3) unique in its emission wavelength, (4) durable, (5) environmentally rugged and safe to personnel and equipment, and (6) amenable to fabrication in a variety of shapes and sizes. The concept is applicable to both silicate- and phosphate-based glasses.

\section{Mission Relevance}

The new materials developed in this effort will provide a unique approach to evaluating potential applications in support of radiologically safe products, such as radio-luminescent (i.e., self powered) lightweight marking and signaling media, egress-marking additives, paint, and other applications. Interest in such radioluminescent materials is extremely high within the Department of Defense, Department of Justice, the Department of Homeland Security, and other agencies for the purpose of monitoring individuals, high-value assets, radioactive material, and military personnel.

\section{Results and Accomplishments}

A series of lithium silicate glasses doped with Nd (neodymium) was prepared with varying neodymium activator concentrations that covered a compositional range extending from a fraction of a percent of $\mathrm{Nd}$ to several percent. These samples were subsequently characterized in a series of optical experiments with the goal of identifying the $\mathrm{Nd}$ concentration that yielded the optimum luminescence output. Optical absorption and other optical measurements were performed in the wavelength region extending from 180 to $3000 \mathrm{~nm}$, and the $\mathrm{Nd}^{3+} \mathrm{f}-\mathrm{f}$ transitions from the ${ }^{4} I_{9 / 2}$ level to the Nd-excited states were identified. The spectral data were used to plot the absorption coefficient (in $\mathrm{cm}^{-1}$ ) as a function of the $\mathrm{Nd}$ concentration present in the glass melt. Direct observations of the infrared (IR) luminescence output were also made for the series of samples using an image intensifier equipped with a narrow-pass IR filter. These observations clearly identified those glass samples that contained a narrow range of $\mathrm{Nd}$ concentrations that yielded the most intense IR output when excited by broad wavelength visible light. These observations have restricted the $\mathrm{Nd}$ concentration to a range that will now be examined in detail 
using an integrating sphere system in order to obtain quantitative light yield results and, thereby, further refine the value for the $\mathrm{Nd}$ concentration that results in the optimum performance in terms of light output of the doped lithium silicate glass. Several hundred grams of lithium carbonate containing isotopically enriched ${ }^{6} \mathrm{Li}\left(\sim 95 \%{ }^{6} \mathrm{Li}\right)$ were obtained, and this material is currently being chemically purified by Nucsafe, Inc., using ion-exchange methods. These specimens will then be employed in the second phase of the investigations designed to yield a new type of radioluminescent glass.

Significant delays in this effort were encountered during the past year due to the long downtimes at HFIR for maintenance, Cold Source design, installation, and testing. The more than 1 year hiatus and new experiment authorization basis also resulted in long experiment queues. The remaining work on this effort is expected to be completed in the next 6 months barring any unforeseen problems at HFIR.

The ${ }^{6} \mathrm{Li}$ was purified and delivered to ORNL. Several lithium silicate glass samples of varying compositions and ${ }^{6} \mathrm{Li}$ were produced. Two different lithium silicate glass samples were prepared for irradiation in the HFIR hydraulic tube facility. The hydraulic tubes have been fabricated, tested and are awaiting approval for irradiation in HFIR. Additional glass samples are scheduled for irradiation in the HFIR pneumatic tube facility and the gamma irradiation facility.

\section{5: Exploring Layered Materials with Neutron and Photon Spectroscopy To Determine the Depth and Water Content in Subsurface Layers of Planets}

Hatice Akkurt

\section{Project Description}

The objective of this project is to investigate the feasibility of using neutron detection and photon spectroscopy methods to determine the composition and thickness of layered materials. Since the heterogeneous layering problem is very difficult to solve in a general sense, initially, the specific focus of this project was to develop a method for identifying regions with water and determining the depth and concentration of water in subsurface layers. The primary application of the method was water detection, and hence life detection, in lunar and planetary exploration. The project was conducted in two phases. First, an algorithm was developed to determine the depth and water content in subsurface layers. For this task, many simplifying assumptions were made to reduce the number of unknowns. Then, this algorithm was improved to solve the more complicated problem of determining the composition of the top layer in addition to the water content and depth of the bottom layer. Hence, one of the severe limiting assumptions is eliminated. For this project, a significant number of Monte Carlo simulations were performed for forward calculations, and an algorithm was developed to invert the problem to determine the unknowns. A series of measurements were performed to demonstrate the advantages of using pulsed neutron generators (PNG) coupled with neutron and photon detectors for planetary characterization purposes. 


\section{Mission Relevance}

The immediate application of this project is lunar and planetary characterization; hence, the main agency that could benefit from this project is the National Aeronautics and Space Administration (NASA). The detection and characterization of water-rich regions on planets has been of great interest to NASA. Because of the President's exploration initiative that includes returning astronauts to the moon, NASA has considerable interest in determining the presence of water/ice in the permanently shadowed regions near the poles. The principal investigator participated in the preparation of a proposal, lead by the NASA Goddard Space Flight Center (GSFC) in collaboration with Schlumberger and several universities, for the Mars-Scout mission. Although that proposal was not funded, submitting a very similar proposal for the upcoming missions to the moon and Mars is currently under consideration.

Since the ultimate goal of this project was to perform environmental surveying, either remotely or in the field on Earth, its moon, and on other planets, this would be particularly useful for environmental monitoring - looking for subsurface contaminant layers or looking for subsurface minerals. Therefore, we expect that other organizations will be interested in this technology including the Department of Energy (DOE) for waste monitoring, the Department of Homeland Security (DHS), and industry.

\section{Results and Accomplishments}

The efforts on the algorithm development continued during the second, final, year of this project. To date, several approaches have been developed to determine the depth of the top layer. These methods include: (1) using a database approach combined with neural networks, (2) finding the solution using an iterative approach based on bisection method, (3) using analytical solutions based on time-dependent transport and diffusion equation. Despite the significant efforts to have a practical and robust algorithm, all the approaches that have been developed so far suffer from being either computationally expensive or yielding non-unique solutions. Although finding the depth required extensive calculations, after depth is determined, the next phase of the project, which is the identification and quantification of all the nuclides in the soil, including water, is achieved easily by modifying the previously developed fixed-point algorithm.

A series of measurements was performed at Princeton to demonstrate the advantages of using an instrument package consisting of a PNG coupled with neutron and photon detectors for planetary characterization purposes. Although the measurements were performed by the researchers from GSFC and Schlumberger, ORNL was involved in the planning, design, and analysis of the measurements. The measurements demonstrated that a combined PNG/neutron and photon detector system has the potential to provide significant geochemical results on planetary surfaces. The ability to separate the inelastic and capture spectra when using a scintillation spectrometer greatly improves the ability to extract quantitative information from the measurements. The capture spectra and neutron detector results are extremely sensitive to variations in concentration and spatial distribution of water and to the detection of other elements with large capture cross sections, like chlorine. These measurements were also benchmarked against computations using the Monte Carlo code, MCNPX (Monte Carlo N-Particle eXtended), to demonstrate that good agreement can be achieved. For die-away measurements, there is a good agreement between the computational model and measurement. Several publications, 
including a journal article, are in preparation to report the results of the measurements and the benchmark computations.

The project successfully demonstrated the usefulness of an instrumentation package based on PNG combined with neutron and photon detectors for planetary characterization purposes. Currently, all indications show that NASA is seriously considering the use of such an instrumentation package in future missions. Unfortunately, the project had a somewhat limited success for the development of a practical and robust algorithm for a very difficult problem given the current computing resources. However, significant potential for future development exists provided the funds could be secured successfully with future proposals to potential sponsors.

\section{Publications}

Starr, R., et al. 2007. "Combined Gamma-Ray Spectrometer and Pulsed Neutron Generator System for In-Situ Planetary Geochemical Analysis." 38th Lunar and Planetary Science Conference, March 12-16, 2007, League City, TX, USA.

\section{8: Carbonate Thermochemical Cycle for the Production of Hydrogen}

Juan Ferrada, Jack Collins, Les Dole, Charles Forsberg, M. Jonathan Haire, Rodney Hunt, Ben Lewis, Ray Wymer, and Jennifer L. Ladd-Lively

\section{Project Description}

ORNL has developed and demonstrated a novel carbonate thermochemical cycle for the production of hydrogen. This thermochemical cycle has a small number of process steps that are all spontaneous at relatively low-temperatures and pressures. Uranium is used at temperatures well below $700^{\circ} \mathrm{C}$ to decompose water $\left(\mathrm{H}_{2} \mathrm{O}\right)$ to produce hydrogen $\left(\mathrm{H}_{2}\right)$. To accomplish this, steam and other gases are passed through a mixture of uranium oxides and other chemicals at temperatures of $625^{\circ} \mathrm{C}$ and above. With these noncorrosive reagents and relatively mild temperatures and pressures, the processing equipment and the construction materials for the required unit operations are commercially available off the shelf. This thermochemical cycle has several advantages over competing processes because the chemical mechanisms proposed require no inventory of volatile hazardous chemicals and have operating temperatures that are compatible with common materials of construction. This work has (a) corroborated the key postulated mechanisms, (b) conducted a preliminary analysis of the process efficiency, and (c) established the technical and environmental viability of the process.

\section{Mission Relevance}

This project directly supports the Presidential Initiative on the development of a hydrogen economy. Replacing fossil fuels will require massive quantities of hydrogen to supply fuel for cars and other industrial purposes. The U. S. Department of Energy (DOE) Hydrogen program 
will benefit the most from the success of this project. It is generally accepted that thermochemical cycles are the most efficient methods to produce hydrogen at large scale. This uranium-based thermochemical process can use heat provided by nuclear reactors and/or solar collectors, making it an environmentally acceptable bulk-hydrogen source. Therefore, DOE's Nuclear Hydrogen Initiative and the Energy Efficiency and Renewable Energy (EERE) Hydrogen, Fuel Cells and Infrastructure Technologies program will benefit from the development of this process. The Department of Defense also benefits from the success of this project because of its critical need for hydrogen to feed fuel cells and serve as an alternative fuel for battlefield vehicles.

\section{Results and Accomplishments}

Several experiments were performed during FY 2007 that demonstrated the production of hydrogen using uranium oxides as the starting material. In addition, thermodynamic calculations were made based on possible reaction equations for various uranium oxides to assess which oxides can produce the most hydrogen.

This work has demonstrated the overall feasibility of the carbonate thermochemical cycle using common laboratory equipment. Experiments were conducted that verified forward and back reactions (all steps after the forward reaction have been demonstrated on an industrial scale) in a series of reaction stages. The process is fed with water, and it comprises five stages in which the hydrogen is produced in the first stage and the recycle of the uranium compounds and production of oxygen are achieved in the other four. In addition, thermodynamic calculations have verified the feasibility of the expected chemical reactions and have predicted the overall efficiency to range between 30 and $70 \%$. We will conduct additional experiments to further validate the process steps for the remainder of this project.

Patent application US11874958 has been filed for this process. In addition, discussions are under way with the DOE EERE program office to seek follow-on funding for this work. A commercial firm is also interest on this technology.

\section{1: Fuel Pellet Irradiation Capsules for Advanced Reactor Fuel Testing in HFIR}

Joel McDuffee, Ronald Ellis, Jess Gehin, Randy Hobbs, and Larry Ott

\section{Project Description}

The Global Nuclear Energy Partnership (GNEP) is a major U.S. initiative designed to address two key barriers to full development of nuclear power: (1) how to use sensitive technologies in a way that protects global security and (2) how to dispose of the waste safely. As part of this initiative, the United States is proposing the development of a fast reactor that will be used to both produce energy and consume long-lived radioactive transuranics in spent nuclear fuel. Unfortunately, no fast reactors are currently operating in the United States that can be used to test these new fuel configurations in a fast neutron environment. Therefore, DOE is preparing to 
make significant investments in the development of a fast-flux irradiation facility. HFIR should be considered for this mission, and may have an advantage over the other concepts in terms of cost, schedule, and capability. The purpose of this project is to (1) determine GNEP fuel irradiation projects that are best suited for HFIR and (2) perform a preliminary evaluation of HFIR for use as a fast irradiation facility.

\section{Mission Relevance}

This project will directly support the implementation of the GNEP program, which supports national security by discouraging other nations from developing uranium enrichment capabilities. More directly, this project will also reduce the number of international shipments of nuclear fuel. Proposed Advanced Burner Reactor (ABR) fuel must be tested in an irradiation environment; however, the United States does not currently have any operating facilities that can produce a fast reactor spectrum. This project may allow the United States to at least partially qualify ABR fuel using a domestic resource.

DOE has not yet selected an irradiation facility. However, this project allowed a team from ORNL to present a conceptual design to DOE's expert evaluation committee in Salt Lake City on October 11, 2007. ORNL's design was well received by the committee. We believe that ORNL is in excellent position to secure this project.

\section{Results and Accomplishments}

Three fuel irradiation concepts were evaluated for this project: (1) a fuel-boosted concept located in the reflector, (2) a single-pin concept in the flux trap, and (3) a tri-pin concept in the flux trap. All concepts included a $\mathrm{Eu}_{2} \mathrm{O}_{3}$ thermal neutron shield to absorb thermal neutrons.

For the fuel-boosted concept in the reflector, the project team concluded that, while booster fuel plates do increase the fast neutron flux, the increase is insufficient to meet the GNEP goal of $>1 \mathrm{E}+15 \mathrm{n} / \mathrm{cm}^{2}$-sec.

Both single-pin and tri-pin flux trap concepts are capable of meeting or exceeding the fast flux criterion. However, the project team concluded that the single-pin concept was inferior because (1) it is much more difficult to cool, (2) the fuel pin and shield cannot easily be separated, and (3) it has a higher reactivity per fuel pin.

The project team selected the tri-pin flux trap concept as the best arrangement for fast-spectrum fuel irradiations. This concept has ample cooling capability, and the thermal shield assembly is completely separate from the fuel pin assembly. This allows fuel and shield replacements to occur at different intervals. Multiple irradiation assemblies may be possible if care is taken to ensure that the power tilt criteria remain within tolerable limits and additional measures are taken to offset the reactivity load associated with the thermal neutron shields. 


\section{2: Generalized Perturbation Methods for Transport Computations with Unstructured Meshes}

Mark L. Williams and Mark D. DeHart

\section{Project Description}

Generalized perturbation theory (GPT) is a technique used for sensitivity and uncertainty (S/U) analysis of calculated reactor safety and performance parameters. S/U analysis provides a rigorous approach to quantify design tolerances and operating limits for advanced reactors, for which little or no experience database exists. Because DOE's national nuclear energy initiatives rely heavily on advanced computer simulations, it is imperative to determine realistic uncertainties in calculated predictions.

This Seed project demonstrates the feasibility of developing a GPT methodology based on ORNL's advanced reactor lattice code NEWT, which solves the neutron transport equation for complex geometries modeled by polygons in a two-dimensional, unstructured solution mesh. NEWT is a module in the widely used SCALE computer code system. Although SCALE has the capability to perform $\mathrm{S} / \mathrm{U}$ analysis of the critical multiplication factor, it does not address other important reactor parameters which require GPT. This work represents the first time that GPT has been studied for deterministic transport solutions on an unstructured mesh. The combination of GPT and high-fidelity modeling of reactor lattice components means that sensitivities of neutronics phenomena such actinide conversion ratio, reactivity feedback, and power peaking can be examined with unprecedented spatial and energy detail.

\section{Mission Relevance}

In outlining developmental activities for the Global Nuclear Energy Partnership (GNEP) and the Advanced Fuel Cycle Initiative (AFCI), DOE's Office of Nuclear Energy and Office of Science recently identified $\mathrm{S} / \mathrm{U}$ methods as a critical need for quantifying the impact of data uncertainties on reactor design calculations, and for reducing these uncertainties. The $\mathrm{S} / \mathrm{U}$ methods studied during the Seed money project could address these needs for advanced core designs and for criticality safety applications in fuel reprocessing/fabrication facilities proposed for the GNEP and AFCI programs. However, due to ongoing uncertainty regarding the GNEP budget, we have not pursued DOE funding to further expand this research in FY 2008. Instead, a proposal has been made to the Atomic Energy of Canada, Limited (AECL) to utilize GPT methods for design of their Advanced CANDU Reactor (ACR). AECL is currently funding ORNL to apply previously available S/U techniques in SCALE for ACR calculations, and they have expressed a strong interest in exploring GPT techniques during the next year.

\section{Results and Accomplishments}

The following goals of the proposal were accomplished: (1) solution of 1D generalized adjoint equation, (2) solution of 2D equation for unstructured mesh, (3) prototypic routines to compute GPT sensitivities with implicit perturbationsc and (4) sample calculations. 
Example reactor physics calculations were performed for several cases, showing that GPT can address questions such as the following: (a) What is the peak pin-power uncertainty due to nuclear data uncertainties? (b) Which data have the greatest impact on this uncertainty? (c) What is the sensitivity of the conversion ratio to fuel enrichment and coolant density? (d) How sensitive are in-core detector responses to the power in different pins? Among the interesting discoveries made has been the importance of perturbations in resonance self-shielding ("implicit effects") for reaction ratio responses in thermal systems. The GPT technique developed here is the only one that treats these effects.

In the area of criticality safety, ORNL previously developed S/U tools to reduce uncertainty in the calculated subcriticality margin of a fissionable configuration, by using critical benchmark experiments. The current tools consider only multiplication factors for critical benchmarks, but this work has demonstrated that with further development, GPT methods could be used to include measured reaction rates in the procedure, thus improving safety validation analysis.

In summary, all objectives of the Seed money project were achieved. The project established the feasibility of using GPT methods based on 2D unstructured meshes. A prototypic computational procedure was developed and used to demonstrate potential applications of the methodology. Several important areas for further development were identified. A paper describing the work has been submitted to the 2008 International Conference on Physics of Reactors. One potential sponsor (AECL) has expressed an interest in supporting additional GPT work during the next year.

\section{6: Intensified Continuous Production of Biodiesel}

Costas Tsouris, Joanna McFarlane, and Joe Birdwell

\section{Project Description}

Based on the well-known chemistry of catalytic transesterification of triglycerides to produce biodiesel, this short Seed project-with a total budget of $\$ 28,000$ - focused on a proof-ofprinciple study to determine whether biodiesel could be produced using a centrifugal contactor. The centrifugal liquid-liquid contactor has been under development at ORNL and other DOE national laboratories for nuclear spent fuel reprocessing via liquid extraction since the 1970s. The study was divided into three steps: (1) Ensure that the products of the transesterification reaction, including the biodiesel phase and the glycerol phase, have enough density difference, so that their emulsion could be separated by a centrifugal contactor. (2) Determine whether the kinetics of the transesterification reaction is fast enough, so that the reaction would be completed within the residence time allowed by the centrifugal contactor. (3) Demonstrate continuous production of biodiesel using a centrifugal contactor that performs both chemical reaction and phase separation in a single unit operation. A centrifugal contactor was employed in steps 1 and 3 , while in step 2, a homogenizer was used to remove interface mass-transfer limitations, so that the intrinsic kinetics can be obtained. The ASTM method D6584-07 employing gas

chromatography was used to analyze liquid samples. 


\title{
Mission Relevance
}

Biodiesel is touted as a means to diversify our supply of transportation fuel, addressing the President's goal of reducing U.S. dependence on nondomestic suppliers by $20 \%$. For a number of reasons ranging from production to end-use issues, biodiesel represents only a small fraction of the transportation fuel used in the United States. This project addressed the aspect of biodiesel production that limits synthesis to a slow batch process. Conventional production methods are batch in nature, apparently based on the assumption that the rates of the key chemical reactions are slow. Our hypothesis was that the reaction kinetics for the transesterification of triglycerides is sufficiently fast, particularly in an excess of catalyst, and that interfacial mass transfer and phase separation control the process. By proving this hypothesis, an intensified two-phase reactor may be utilized to greatly increase biodiesel production rates by increasing interphase transport and phase separation. Thus, this work is directly relevant to the DOE national energy security objectives in that it supports the development of an alternative energy source.

\section{Results and Accomplishments}

The results of all three steps of the project were successful. In step 1, the transesterfication reaction of vegetable oil was initially performed in a stirred tank at $55^{\circ} \mathrm{C}$. A long residence time allowed the reaction to be completed. The emulsion product was then fed to a 5-cm-rotor centrifugal contactor and at the exit of the contactor pure phases were received. Thus, it was concluded that the density difference of the methyl-esters phase and the glycerol phase was sufficient for the separation to be performed by the centrifugal contactor in a matter of seconds. In step 2, where a liquid homogenizer was employed to carry out the mixing and reaction of the reagents, samples analyzed by gas chromatography showed that the reaction is completed within one minute. In step 3, modifications in the operating procedure of a centrifugal contactor were made to allow a residence time of one minute. It was then demonstrated that the continuous-flow centrifugal contactor could be used for simultaneous reaction and phase separation in biodiesel production. This accomplishment reduces the production time of biodiesel from hours required by batch processes to minutes. Intensified continuous production of biodiesel, where a single, compact unit operation performs simultaneously multiple chemical processes in a continuous mode has therefore been demonstrated in this project.

\section{8: Molecular Engineering of Core-Shell Interfaces toward Controllable Production of Brighter, Optically Tunable Quantum Dots}

\author{
Michael Z. Hu, Reza Dabestani, and Lei Shao
}

\section{Project Description}

Quantum dots (QDs) (semiconductor nanocrystals) have been extensively investigated due to size-dependent quantum confinement effects and applications to biomedical labeling, molecular imaging, solar cell, lasers, light-emitting diodes, and coding materials for soldiers, etc. Core-shell QDs are preferred because a semiconductor core coated with a larger band gap shell offers the composite better stability and optical properties. The objective of this project is to demonstrate a 
novel "molecular engineering" approach to create innovative core-shell QDs that could provide higher quantum yield (thus, higher stability and brightness) and to enable a precise control of the optical coupling and decoupling properties of the core-shell structure. The proposed approach could allow precise tailoring of the core surface with conjugated molecules in the form of selfassembled monolayers (SAM). The SAM-modified surfaces can be designed to grow or link a wide range of inorganic shell materials, with better shell uniformity and well controlled nanostructures. In addition, SAM layer thickness and functionality can be adjusted by design to enable optical coupling/decoupling between core and shell. Proof of principle of the proposed approach could lead to a route to massively produce high-quality and reproducible QDs, a long sought-after goal in this field.

\section{Mission Relevance}

This fundamental work on core-shell QD structures could impact many applications, including solar cells, biomedical imaging, biological labeling, anticounterfeiting, military, homeland security and counter espionage, LEDs, and nonlinear optics. Upon proof of principle of higher brightness and dual-wavelength emissions from a single core-SAM-shell QD, this work will attract research funding and collaborations from DOE and other federal agencies, including the National Institutes of Health (NIH), Department of Defense (DoD), National Aeronautics and Space Administration (NASA), and Department of Homeland Security (DHS), as well as industry. For example, the more stable QDs will clearly benefit DOE's initiatives in solar cells. Our research will strengthen ORNL's position as a leader in nanomaterials and nanotechnology and as an innovator in the application of nanotechnologies to energy, biomedical, biology, and security.

\section{Results and Accomplishments}

During the past 6 months, we have focused our research on the chemical syntheses and material/optical characterization of core-shell QDs with unique structures. Synthesis of optically stable CdSe/ZnS QDs is a fundamental but essential step to demonstrate a molecular tuning effect on fluorescence resonance energy transfer (FRET) between QDs or between the core and shell of QD materials. To conduct FRET studies using CdSe/ZnS as an applicable QD unit, 3-5 monolayers of $\mathrm{ZnS}$ are needed, which is beyond current synthetic capabilities. In collaboration with Steacie Institute, we have developed a new mixed-solvents synthesis approach that shows possibility of producing QDs with thicker shell layers of $\mathrm{ZnS}$.

The observation of CdSe/ZnS QDs by a high-resolution aberration-corrected electron microscope revealed that these core-shell QDs exhibit nonepitaxial structures with the appearance of amorphous surface constituents, a phenomenon that has never being reported before, and its cause is unclear.

We have made significant progress toward the creation of novel molecularly linked/interspaced QDs structures (QD-molecule-QD), which are critical to the proof of principle on tuning QD fluorescent properties by varying molecular spacing between QDs or between core-QD and shell-QD. The images that we have obtained by scanning transmission electron microscope may be the first observed QD structure with organic molecules coupling the core and the shell. Future studies will focus on controlling the interspacing by varying the length of molecular linkers and 
tailoring the cluster size of molecularly linked QDs, as well as on confirming the optical tunability by the fluorescent emission spectra.

\title{
00450: Development of a Hybrid Computational Phantom Model
}

\author{
Hatice Akkurt and Keith F. Eckerman
}

\section{Project Description}

The first heterogeneous computational phantom models of the human anatomy were developed at Oak Ridge National Laboratory (ORNL) over three decades ago. In these phantoms, the organs were described by simple mathematical equations to facilitate radiation transport calculations. They became the standard for the assessment of organ doses due to internal or external exposure to radiation in medical, occupational, and environmental radiation protection. Recently, medical image data have been used to construct voxel phantoms (i.e., phantoms constructed using a fine mesh based on imaging data). In these phantoms, the organs are represented in great detail and realism; however, computational challenges (e.g., increased computational times and memory requirements), as well as major difficulties in modeling realistic exposure configurations, are noted in their application. To address these issues, this proposal introduces the concept of a hybrid phantom-a voxel representation in areas where detail is required (e.g., the head and torso) coupled with mathematical descriptions, where a high degree of detail is not required and flexible modeling of the configuration is desired (e.g., the arms and legs). This project investigates the feasibility of this approach for radiation dose assessment.

\section{Mission Relevance}

The project is relevant to DOE national security missions. Specifically, the DOE occupational worker dose assessment program could benefit from the project for the assessment of organ doses in realistic exposure configurations. In addition to the occupational exposure program, national security could benefit from the evaluation of doses in the case of a radiological terrorist event.

The project has potential benefit for several agencies, including the Environmental Protection Agency (EPA), Centers for Disease Control (CDC), Nuclear Regulatory Commission (NRC), and Department of Homeland Security (DHS). For these agencies, the ability to estimate the radiation doses for realistic configurations in a practical manner is important-not only for occupational exposure - but also for the public in the event of an occurrence such as a nuclear accident or terrorist incident. The National Institutes of Health (NIH) is another agency that could potentially benefit from this work-since the possibility of reconstructing the whole body from partial computed tomography (CT) scans using hybrid approach is especially important for the investigation of secondary cancers. The National Aeronautics and Space Administration (NASA) will potentially benefit from this work for estimating organ doses in realistic scenarios and configurations for astronauts for future manned missions to the Moon and Mars. 


\section{Results and Accomplishments}

Significant progress has been made during the initial year of this project. A computational model for the fully voxelized phantom has been identified and received. In the current voxel model, the phantom was enclosed in a box that is divided into voxels (fine meshes). There are many empty unnecessary voxels outside the body parts that are filled with air. In order to reduce the number of voxels, the phantom was first divided into parts, and the head and upper and lower parts of the torso were enclosed in appropriately sized boxes. Then, this model was revised to develop the hybrid phantom model in which the voxel-represented arms and legs were replaced with a mathematical description. During this modification, the shapes of the arms and legs were kept as realistic as possible, and special attention was given to conserve their volumes. By using the hybrid approach and using varied box sizes for different parts of the body, the number of voxels was reduced from 7 million to 3 million. Subsequently, the computational time and memory were reduced by almost $50 \%$. The organ dose values for the original voxel and the hybrid phantom were computed using MCNPX (Monte Carlo N-Particle eXtended) and confirmed to be in agreement within the statistical uncertainties.

The revisions to the developed hybrid computational phantom model will continue further next year to add freely moving capabilities (i.e., movement of arms and legs) for the assessment of radiation dose for realistic exposure configurations.

\section{Publications}

Akkurt, H. and K. F. Eckerman. 2007. "VOXMAT: Hybrid Computational Phantom for Dose Assessment." American Nuclear Society Annual Meeting 96, 642-643. 


\section{7: Big Bang Cosmology and Online Simulation Suite}

Michael Smith, Eric Lingerfelt, Jason Scott, W. Raphael Hix, Caroline Nesaraja, George Fuller, and David Tytler

\section{Project Description}

One of the fastest growing and most widely popularized (e.g., Nobel Prize in 2006) research field in physics is cosmology - the study of the birth, structure, evolution, and fate of our Universe. One powerful approach in cosmology is to calculate the amount of light elements (e.g., hydrogen, helium, lithium) synthesized in the early Universe and, through comparison with observations, put limits on such crucial parameters as the total amount of "normal" matter in the Universe. However, while there are thousands of cosmologists who need this information, only a few have the access to specialized codes to follow this approach. We have created a working prototype of the world's first suite of online interactive computer codes that enables researchers around the world to run these important calculations. Our codes enable users to customize the calculations and share their results with colleagues. We will also improve the input to existing Big Bang codes by assessing the latest information on thermonuclear reactions that form light elements in the Big Bang and run our suite of codes to put new constraints on the matter density of the Universe.

\section{Mission Relevance}

Our overall goal is to launch a program in cosmology research at ORNL. This program would involve numerous aspects, including improving the nuclear physics input to Big Bang calculations, enabling calculations of nonstandard Big Bang models, investigating the role of exotic particles in the early Universe, and maintaining and developing our software suite. In the DOE Office of Sciences, the Office of High Energy Physics supports research into the origin, properties, and interactions of elementary particles and other basic constituents of matter, and the Office of Nuclear Physics supports research into the nature of matter and energy, including astrophysics and the early Universe. Both offices have expressed interest in our research program. The National Aeronautics and Space Administration (NASA) sponsors research into cosmology and the early Universe - and its Applied Information Systems Research program sponsors the development of software systems that support NASA science goals. Finally, the National Science Foundation (NSF) Division of Astronomical Sciences also sponsors research in cosmology and the early Universe through its astronomy and astrophysics research grants. Both NASA and NSF have stated that this research is appropriate for funding through their programs.

\section{Results and Accomplishments}

In FY 2007, we completed a prototype of our "bigbangonline" software suite. Users of this system, freely available at bigbangonline.org, can specify the latest set of input nuclear physics and cosmological parameters to set up their custom early Universe simulation, and the latest primordial abundance observations to determine the constraints on cosmology parameters. The suite features excellent visualization tools to completely customize publication quality plots, and 
enables sharing of simulation results among users. We also completed the assembly of the bigbangonline.org website to serve as a gateway to the codes and a repository of information on Big Bang and early Universe studies. We prepared and submitted a \$2.9M Work for Others proposal to NASA Applied Information Systems Research Program (AISRP) for follow-on work. The proposal received the highest ratings, "Excellent," in all categories, but was not funded because of budget restrictions. The proposal will be resubmitted in the next available call. Additionally, a follow-on funding proposal to the DOE Office of High Energy Physics is in preparation.

\title{
00455: Computing the Electric Dipole Moment of the Neutron and the Schiff Moment of the Nucleus
}

\author{
D. J. Dean and M. Ramsey-Musolf
}

\section{Project Description}

This project begins a theoretical program in "beyond the Standard Model physics" which will closely tie to the new experimental efforts at the Fundamental Neutron Physics Beam (FNPB) at the Spallation Neutron Source (SNS). The project allows initial progress on two problems relevant to experimental effort at ORNL (a new neutron electric dipole moment measurement and parity violating neutron scattering measurements) being developed at the SNS.

\section{Mission Relevance}

The operation of the FNPB at the SNS represents a new forefront in nuclear physics research funded by the Office of Science Nuclear Physics program. The high intensity source of monochromatic neutrons provided by this facility will allow experimental nuclear physicists to perform measurements of fundamental properties of the neutron and its interactions with unprecedented precision. These experiments will test basic features of the Standard Model of the electroweak and strong interactions and search for evidence of physics going beyond the Standard Model. This world-leading effort represents an important and fundamental area of experimental nuclear physics research at ORNL.

A number of theoretical computations are needed to help interpret the FNBP experiments. In the case of the neutron electric dipole moment (EDM) search, the implications of this experiment for different scenarios for charge-parity $(\mathrm{CP})$ violation requires a comparison of the results with complementary searches being carried out for the EDMs of the electron and neutral atoms. A comprehensive phenomenological analysis of these EDM searches in models for new $\mathrm{CP}$ violation remains to be completed. Similarly, new computations of the Schiff moments of neutral atoms are needed. A similar comprehensive analysis of neutron decay parameters and their implications for new physics remains to be performed. Finally, the theoretical framework for interpreting the hadronic PV experiments has recently been reformulated using the methods of effective field theory (EFT). In particular, the sensitivity of the different hadronic PV experiments to the low energy constants in the EFT must be delineated for the broad array of 
measurements that will be performed at the FNPB and elsewhere. In short, there exists an opportunity for significant theoretical advances by performing these computations and analyses.

\section{Results and Accomplishments}

We utilized the initial FY 2007 funding $(\$ 25 \mathrm{~K})$ to hire a post-doctoral associate and to begin the studies. The project should come to fruition during FY 2008 when the bulk of the funding will be used. 



\section{AUTHOR INDEX}

Agamalian, M. 62

Ahmad, Nabeela 109

Akkurt, Hatice

200, 209

Alexander, C. W.

199

Alley, Gary.

159

Allison, David

132

Allison, S. W

46, 156

Anker, J 67

Ankner, John F

60,66

Apra, Edoardo

103

Archibald, Richard .................................. 20

Armstrong Beth L. ................................. 184

Baity, F. Wallace ..................................... 71

Baldwin, Charles A................................. 15

Bank, Tracy L. ..................................... 165

Barrett, Richard F................................... 98

Baskaran, Latha........................... 167, 170

Battiste, Rick....................................... 15

Becher, P. F.......................................... 191

Beckerman, Barbara G......................... 148

Beene James R. ..................................... 117

Bell, Gary L. .......................................... 15

Bennink, R...................................... 114

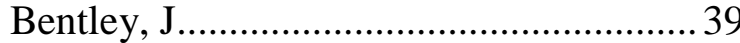

Bernholc, Jerzy ...................................... 103

Berry, L. A. ............................................. 173

Besmann, Theodore .............................. 112

Bhatia, Nikhil ........................................ 111

Binder, Jeffrey L. ................................... 15

Birdwell, Joseph............................20, 206

Blau, Peter...................................... 52, 193

Boatner, L. A................................... 46, 199

Bonnesen, Peter V.................................. 38

Bordner, Andrew............................ 100, 109

Borisevich, Albina Y. ............................. 33

Borole, Abhijeet P....................... 89, 133

Brady, Michael P. ........................ 182, 192

Brandt, Craig C..................................... 129

Brightwell, Ron.................................... 106

Britt, Phillip F. ............................... 25, 26

Britton, C. L......................................... 160

Brown, G.M. ...............................35, 141

Brown, S. D.......................... 73, 85, 129
Buchanan, A. C...................................... 190

Buckner, Mark A. .....................................53

Budai, John D........................................ 178

Carroll, S.............................................. 85

Chakoumakos, Bryan.............................6 69

Chakravarthy, Kalyan .............................. 10

Cheng, Meng-Dawn ............................... 168

Chesler, Elissa.................................. 74, 82

Chialvo, Ariel A................................ 28, 42

Chinthavali, Madhu.................................. 18

Chisholm, Matthew F...................... 34, 187

Choi, Jae-Soon ........................................ 154

Christen, Hans .............................. 151, 196

Clarno, Kevin ............................... 95, 112

Classen, Aimée T....................................79

Cobb, John W........................................ 105

Cole, D. R. ....................... 28, 65, 69, 197

Collins, Jack ......................................... 202

Compere, Alicia ..................................... 19

Compton, Robert N................................ 176

Crom, Benjamin C. ................................ 171

Cui, Shengting..........................................20

Cummings, Peter T. ............................... 65

Custelcean, Radu..................................... 38

d'Azevedo, Eduardo F. ............................ 95

D’Urso, B. R................................ 121, 156

Dabestani, Reza........................... 141, 207

Dahotre, Narendra B.................... 184, 193

Dai, Sheng.............................. 9, 26, 37

Dale, Virginia....................................... 170

Daniel, Claus................................. 16, 184

Datskos, Panos ........................ 12, 16, 153

David, Stan A................................52, 57

Daw, Stuart .............................................. 10

de Oliveira, Cassiano R. E. ..................... 95

de Jonge, Niels ................... 23, 33, 87, 179

de Almeida, Valmor .................... 10, 20, 95

Dean, D. J............................................ 212

DeHart, Mark D. .................................... 205

Delmau, Lætitia H................................... 38

DePaoli, David..............................9, 9, 19, 20

Dinwiddie, R. B. ............................ 15, 27

Doktycz, Mitchel........................... 90, 132 
Dole, Les ............................................... 202

Dos Santos, Antonio Moreira ..................69 69

Drake, John ............................................93

Driscoll, Michael E. .............................. 158

Dudney, Nancy ................................ 9, 178

Eckerman, Keith F............................... 209

Efroymson, Rebecca ........................... 164

Eisenbach, M........................................ 108

Ellis, Ronald.......................................203

Engelmann, Christian............................ 106

Engle, N. L........................................... 85

Eres, Gyula......................24, 37, 176, 196

Erickson, David.......................................93

Ericson, M. Nance...........................90, 158

Evans, B. M., III........................... 156, 159

Evans, Barbara R.

Evans, P.

$139,139,142$

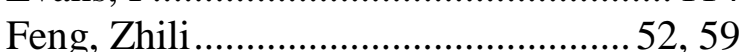

Fernandez-Baca, Jaime A. ......................59

Ferrada, Juan ........................................ 202

Fields, Matthew.................................... 165

Fisher, Galen B. ................................... 154

Fishman, R. S...................................... 185

Foote, Linda .......................................... 86

Forsberg, Charles ................................. 202

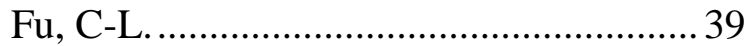

Fuller, George ....................................... 211

Ganguly, Auroop R................................ 43

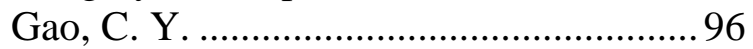

Gao, Yanfei ........................................... 31

Gardner, Timothy................................... 158

Garten, Charles T.................................. 170

Gauld, Ian............................................. 112

Gehin, Jess .......................................... 203

Geohegan, David B........................ 24, 122

Goeringer, Douglas E.............................. 30

Gorin, Andrey ............................. 100, 109

Gossage, David ...................................... 168

Gouge, Mike .......................................... 12

Goulding, Richard H............................ 71

Goyal, Amit............................ 12, 31, 175

Grandas, Oscar, MD ............................ 148

Green, Johney B............................ 10, 154

Greenbaum, Elias .................................... 138

Grice, Warren P. ......................... 114, 117
Griest, Wayne H................................... 74

Griffin, G. D....................................... 156

Griffith, William .................................. 19

Gruszkiewicz, Miroslaw S.....................28

$\mathrm{Gu}$, Baohua .......................... 67, 132, 176

$\mathrm{Gu}$, Jessie .............................................. 109

Gunter, Lee ..................................... 77, 86

Hagaman, Edward................................... 24

Haire, M. Jonathan ............................... 202

Hardy, J. E............................................ 160

Hargrove, William ................................ 167

Harris, Jeffrey H................................... 13

Harrison, Robert..................................... 103

Hart, Kevin J. ......................................... 43

Hauser, Loren............................................. 43

Havener, Charles C. .............................. 117

Hay, Benjamin P................................... 40

Heatherly, Lee ...................................... 175

Heller, William T.....................................60 60

Herwig, Kenneth W...............................6 65

Hinchee, Maud ........................................ 77

Hively, Lee M. ..................................... 163

Hix, W. Raphael...................................211

Hobbs, Randy......................................... 203

Hoelzer, D. T......................................... 39

Hoffman, Forrest..................................... 93

Hong, Kunlun........................... 25, 26, 141

Horita, Juske ...........................................69

Howe, Jane ............................................ 178

Hsueh, C. H............................................ 191

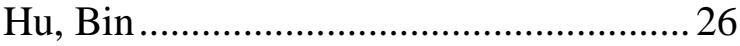

Hu, Michael Z. ...................................... 207

$\mathrm{Hu}$, Zhiyu ................................................. 51

Hubbard, Camden R........................57, 59

Humayun, Mark S............................... 138

Humble, T. ............................................ 114

Hunt, Rodney ........................................ 202

Hurst, Gregory .......................... 73, 85, 86

Ice, Gene E........................................... 180

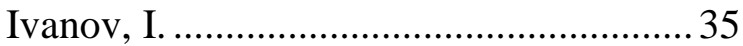

Jackson, Jeremy J................................ 122

Jaeger, E. F......................................... 173

Jager, Henriette ....................................... 164

James, David R................................... 12

Jardine, Philip M.................................... 165

Jawdy, Sara ..................................... 77, 86 
Jellison, J. E 27

Jesse, Stephen ................................ 35, 135

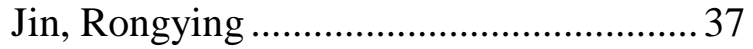

Johnson, Dabney .................................... 82

Johs, A.................................................6 67

Jubin, Robert ......................................... 20

Kaczmarek, Donald..................................77

Kalinin, Sergei V. ................... 35, 135, 151

Kalluri, Udaya ....................................... 86

Kaxiras, Efthimios ............................. 176

Keller, Martin............................... 83, 90

Kendall, Ricky ...................................... 106

Kercher, Andrew ................................... 178

Kertesz, Vilmos................................30, 138

Khamayseh, Ahmed ................................95

Khomami, Bamin...................................20

Kiggans, Jim .......................................... 9

Kilbey, S. Michael, II............................... 66

Kim, Seokho H...................................... 193

King, Anthony...................................... 93

Kisari, P................................................. 35

Kisner, Roger A. ......................................59

Klebig, Mitchell ............................ 82, 128

Klueh, R.L............................................ 62

Kora, Guruprasad .................................. 109

Krause, Herbert F................................. 117

Kruse, Kara L........................................ 148

Kueck, John.......................................... 18

Kulkarni, Nagraj .................................. 159

Kuruganti, Phani Teja .............48, 111, 147

Labinov, S.D. ........................................ 49

Ladd-Lively, Jennifer L....................... 202

Lance, M. J............................................2 27

Lankford, Patricia .................................. 73

Lara-Curzio, Edgar ........................ 15, 52

Larson, Bennett C. ............................... 180

Lavrik, Nickolay ...................... 12, 16, 153

Lee, Ho Nyung...................34, 37, 151, 187

Lee, James W....................................... 139

Levesque, J........................................ 108

Lewis, Ben ........................................... 202

Lewis, Linda ................................46, 199

Lewis, Sam............................................. 10

Li, An-Ping ….................................... 26

Li, Fran.................................................. 18

Li, Yaohang...................................... 100
Liang, Chengdu....................................... 9

Liang, Junjien....................................... 117

Liang, Liyan.......................................... 67

Lingerfelt, Eric ..................................... 211

Littrell, Kenneth C. ................................63

Liu, C. T................................................. 191

Liu, Qing ............................................. 93

Liu, Yie ............................................... 127

Liu, Yuan ............................................... 143

Love, Lonnie J. ................................... 162

Ludtka, Gerard M...................................59

Lupini, Andrew R. ................................. 33

Ma, Xiaosong ....................................... 105

Maccabe, Barney................................... 106

MacIntryre, Lawrence P.........................53

Mackiewicz-Ludtka, Gail ......................59

Mahurin, Shannon................................ 168

Maksimchuk, Anatoly........................... 117

Mamontov, Eugene .................................6 65

Mandrus, David.................................... 188

Martin, S. L. ......................................... 85

Mastalerz, M. ...................................... 197

Mathis, John E. ................................... 196

Maxey, L. Curt ..................................... 154

Mays, Jimmy ..................................25, 66

Maziasz, Philip J................................ 182

McCold Cliff.......................................... 178

McDonald, W. Hayes...............................45

McDuffee, Joel......................................203

McFarlane, Joanna ......................... 10, 206

McKeown, Catherine ............................. 73

McKnight,T. E. ................ 86, 90, 156, 158

Melnichenko, Y. B............................... 197

Messman, Jamie M. ...............................66

Meunier, Vincent .......................... 103, 151

Meyer, Harry ........................................ 161

Michaud, Edward J. ...................... 76, 128

Mielenz, Jonathan ................................. 73

Miller, M .K. ........................................... 39

Mo, Yiming...........................................6 60

Molaison, Jamie ....................................69

Moore, Michael R. ................................ 53

Moore, Tony ........................................... 24

Morrell-Falvey, Jennifer .......................... 86

Morris, Robert N.................................. 15

Moses-DeBusk, M. ............................... 190 
Moyer, Bruce 20,38

Mulholland, Patrick. 167

Munro, Nancy B. 163

Muralidharan, Govindarajan . 18,62

Myles, Dean A. $.60,66,67,68,142$

Namilae, $\mathrm{S}$ 96

Nardelli, Marco Buongiorno 151

Narula, C. K. $19,51,190$

Nesaraja, Caroline 211

Nicholson, D. M. $96,108,159$

Nieh, T.G. 195

Norby, Richard J. .79

Norton, David 137

Nukala, P. K. 96,112

Nutaro, James J. 148

Nutaro, James. 48,147

O'Neill, Hugh $68,138,142$

Oladosu, Gbadebo 164

Ong, Hong 106

Osetskiy, Yu. N. .96

Ostrouchov, George.

Ott, Larry... 112,203

Ott, R. D. .27, 137

Ozpineci, Burak .18

Palanisamy, Giri. .171

Palumbo, Anthony

Pan, Chongle $43,90,128$

Paranthaman, M.P $73,85,109$

Park, Byung-Hoon 12,51

Park, Sea 109

Partridge, William P. ...9

Pawel, Steve 154

Payzant, E. Andrew 193

Pelletier, Dale. 192

Pennycook, Stephen J. 85,86

Perumalla, Kalyan S. .. 33

Pike, Gregory G. 111

Podar, Mircea. .105

Post, Wilfred 83,90

Protopopescu, Vladimir

$\mathrm{Qu}$, Jun

Qualls, A.L. ...93

Radhakrishnan, Bala

Radlinski, A.

Rajic, Slobodan

Raman, Babu
$43,48,111$ .52 49 $57,62,159$ 197 153 73
Ramsey-Musolf, M. ................................. 212

Rao, Nageswara S. ....................................53

Reboredo, F. A....................................... 185

Reilly, Peter T. A. .................................... 145

Reitz, Rolf ................................................. 10

Ren, Weiju ................................................ 62

Rey, Christopher ...................................... 132

Riccuito Daniel .......................................... 93

Rodriguez, Miguel Jr. .................................. 73

Rondinone, Adam J.....................................38

Roth, Philip C............................................ 98

Rother, Gernot............................................ 42

Sabau, A. S.............................................. 27

Sales, Brian C.............................................. 37

Samatova, N. F..........................73, 85, 109

Sanchez, Raul........................................... 118

Sanders, Charlene A.................................. 138

Santella, M. L...................................62, 182

Santodonato, Louis J.................................. 65

Sarma, G. B..................................... 57, 62

Sauers, Isidor............................................... 12

Schadt, Christopher W.................45, 79, 83

Schlom, Darrell G. ……………………........ 34

Schneibel, Joachim H............................... 192

Schulthess, T. C. ………………..... 100, 108

Schultz, David R. ..................................... 117

Schwartz, Viviane ....................................... 16

Scott, Jason ...............................................211

Scott, Stephen L........................................ 106

Seal, K..................................................... 35

Sedowski, Jennifer ......................................74

Shankar, Mallikarjun....................................48

Shao, Lei .................................................... 207

Shaw, Robert W....................... 30, 117, 175

Shelton, William ............................ 103, 151

Shen, Jian ……................................26, 186

Shingledecker, J. P....................................62 62

Simonson, Michael J..................................28

Simpson, John T....................................... 121

Simpson, Michael L. …………………..... 158

Simunovic, Srdjan.................................... 112

Singh, David ..............................34, 188, 192

Sloop, Frederick V., Jr. ............................... 38

Sluder, Scott ............................................... 10

Smith, Barton ................................................ 16

Smith, Greg S............................................... 60 
Smith, Jeremy ....................................... 90

Smith, Michael ........................................ 211

Smithwick, R. W.......................... 46, 199

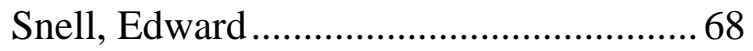

Specht, Eliot D..................................... 180

Standaert, Robert F. ............................... 80

Steele, Bill................................................. 10

Stocks, G. Malcolm................................. 31

Stoller, R. E.................................. 96, 180

Storaasli, O. O...................................... 101

Stovall, John........................................... 48

Stracener, Daniel W.............................. 117

Sumpter, B.G. ............................... 49, 103

Sun, Chengjun...................................... 186

Tao, X................................................ 108

Tao, Xiuping ........................................ 100

Thompson, James R............................. 192

Thundat, Thomas ...........................51, 132

Tian, Yuan............................................ 100

Todd, Peter ............................................. 158

Tolbert, Leon........................................... 18

Tschaplinski, T. J...........................77, 85

Tsouris, Costas ......................... 16, 20, 206

Tulk, Chris A. ......................................... 69

Tuncer, Enis .......................................... 12

Tytler, David ......................................... 211

Umstadter, Donald P............................ 117

Urban, Volker .......................... 25, 38, 142

Vallee, Geoffroy .................................. 106

Van Berkel, Gary ............................ 30, 139

Vane, Charles R. ................................... 117

Vass, Arpad.............................................. 19

Vazhkudai, Sudharshan S. ..................... 105

Veith, Gabriel.................................33, 179

Venkateswaran, Amudhan ...................... 83

Vetter, Jeffrey .............................. 98, 111

Vo-Dinh, Tuan ...................................... 127

Voy, Brynn H........................................ 74

Wagner, Robert ...................................... 10
Walker Frederick J.............................. 180

Wang, Hsin ........................... 52, 159, 188

Wang, John ........................................... 193

Wang, W.............................................. 67

Wang, Wei ................................. 132, 176

Wang, Xun-Li ..........................39, 52, 57

Wang, Yisong ....................... 82, 127, 128

Ward, Richard C. ................................. 148

Warmack, R. J.................................... 160

Weber, Charles....................................... 112

Welton, Robert F.................................... 71

Weston, David.......................................... 86

Whitten, William B......................... 30, 145

Wignall, G. D...................................... 197

Wilgen, John B......................................59

Williams, Mark L.................................205

Wilson, Bruce E.................................... 171

Wilson, Leslie ....................................... 175

Wolfe, Amy.......................................... 170

Woo, Wan C.............................................5 57

Woodward, Jonathan................................ 38

Wymer, Ray ........................................ 202

Xu, Jun ........................................ 137, 143

Yamamoto, Yukinori ............................ 182

Yang, S............................................... 85

Yang, Xiaohan ....................................... 77

Yang, Yunfeng.............................. 85, 130

Yang, Zamin ................................. 83, 130

Yin, Tongming ....................................... 77

Yoon, Mina .......................................... 178

You, Yun..................................... 82, 128

Zhang, Xiaoguang................................ 186

Zhang, Xinye.......................................... 77

Zhang, Zhenyu ...................................... 176

Zhao, Z............................................ 114

Zhong, Jianxin...................................... 31

Zhou, C. .................................................. 108

Zhou, Zhixian......................................... 37 

PROJECT NUMBER INDEX

\begin{tabular}{|c|c|c|c|}
\hline 00001 & 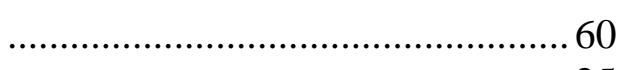 & 00044 & ....... 91 \\
\hline 00002 & 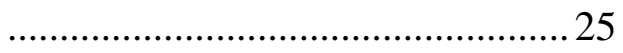 & 00045 & . \\
\hline 00003 & 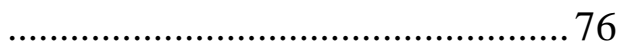 & 00046 & 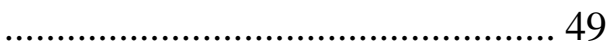 \\
\hline 0004 & $\ldots 62$ & 00047 & \\
\hline 0005 & $\ldots 10$ & 00048 & 118 \\
\hline 00006 & 101 & 00049 & 122 \\
\hline 00007 & …………....... & 00050 & \\
\hline 008 & 103 & 00188 & ...................... 117 \\
\hline 00009 & 105 & 00201 & .. 57 \\
\hline 00010 & 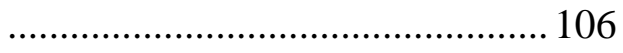 & 00220 & .23 \\
\hline 011 & 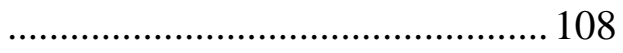 & 00222 & 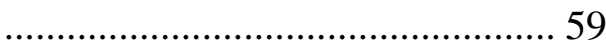 \\
\hline 0012 & 109 & 00223 & . \\
\hline 00013 & 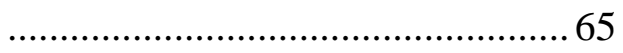 & 00224 & 24 \\
\hline 014 & - & 00226 & \\
\hline 0015 & 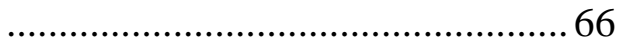 & 00227 & . \\
\hline 016 & - & 00228 & \\
\hline 017 & 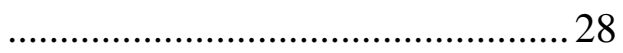 & 0229 & \\
\hline 018 & 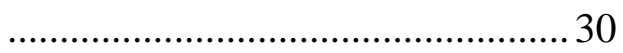 & 00230 & \\
\hline 019 & . & 0231 & …………......... \\
\hline 020 & .85 & 00232 & 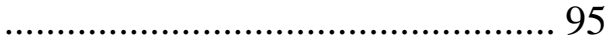 \\
\hline 021 & - & 00233 & . \\
\hline 022 & 68 & 00234 & 12 \\
\hline 023 & - & 00235 & 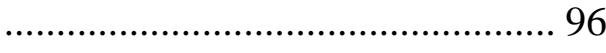 \\
\hline 024 & (2).1.1 & 00236 & 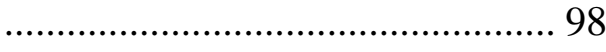 \\
\hline 025 & 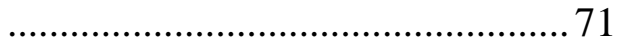 & 00237 & .. 48 \\
\hline 026 & 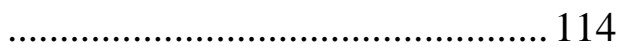 & 00239 & 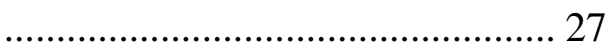 \\
\hline 027 & 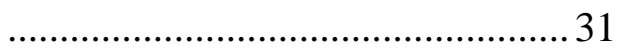 & 00240 & 100 \\
\hline 028 & 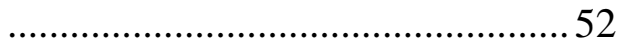 & 00241 & .... 79 \\
\hline 00029 & $\ldots 33$ & 00242 & .... 80 \\
\hline 030 & . & 00243 & ㄱ. \\
\hline 031 & 35 & 00244 & ……………... \\
\hline 032 & 37 & 00245 & 63 \\
\hline 033 & 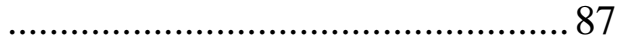 & 00246 & 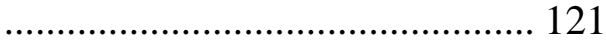 \\
\hline 0034 & 15 & 00356 & 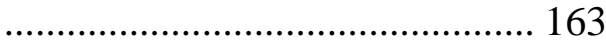 \\
\hline 00035 & 53 & 00371 & 175 \\
\hline 036 & 16 & 00389 & ..... 176 \\
\hline 0037 & 18 & 00394 & 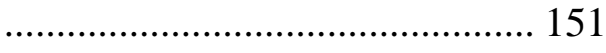 \\
\hline 00038 & ………....... 19 & 00396 & ... 127 \\
\hline 00039 & 20 & 00397 & 147 \\
\hline 00040 & . & 00402 & 128 \\
\hline 041 & . & 00404 & 137 \\
\hline 042 & . 39 & 00405 & ...... 178 \\
\hline 043 & . & 00406 & …………....... 153 \\
\hline
\end{tabular}




\begin{tabular}{|c|c|c|c|}
\hline 00407 & .211 & 00434 & .171 \\
\hline 00408 &. .154 & 00435 & 148 \\
\hline 00409 & .. 156 & 00436 & .159 \\
\hline 00410 & $\ldots 129$ & 00437 &. .160 \\
\hline 00411 & $\ldots .164$ & 00438 & .. 186 \\
\hline 00412 & 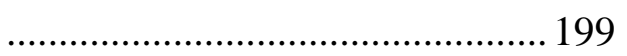 & 00439 & $\ldots 187$ \\
\hline 00413 & $\ldots 165$ & 00440 &. .142 \\
\hline 00414 & $\ldots 138$ & 00441 & $\ldots 173$ \\
\hline 00415 &. .200 & 00442 & .. 132 \\
\hline 00416 & $\ldots 179$ & 00443 & .162 \\
\hline 00417 & $\ldots 180$ & 00444 &. .18 \\
\hline 00418 & ...202 & 00445 & .190 \\
\hline 00419 & $\ldots 167$ & 00446 &. .206 \\
\hline 00420 & ............ 130 & 00447 & $\ldots 191$ \\
\hline 00421 & ….............. 182 & 00448 & ... 207 \\
\hline 00422 & ................... 184 & 00449 &. .192 \\
\hline 00423 & .............. 158 & 00450 & ... 209 \\
\hline 00424 & ... 139 & 00451 & $\ldots 133$ \\
\hline 00425 & $\ldots .141$ & 00452 & $\ldots 197$ \\
\hline 00426 & $\ldots .135$ & 00453 & $\ldots 143$ \\
\hline 00429 & $\ldots 168$ & 00454 & .. 19 \\
\hline 00430 & $\ldots 170$ & 00455 & ... 212 \\
\hline 00431 & ............203 & 00456 & …........................... \\
\hline 00432 & 205 & 00457 & 14 \\
\hline 00433 & 185 & 00458 & 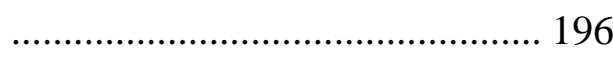 \\
\hline
\end{tabular}

